\title{
Nickel-Catalyzed Directed Cross-Electrophile Coupling of Phenolic Esters with Alkyl Bromides
}

\author{
Feiyan Yang, ${ }^{\text {a }}$ Decai Ding, ${ }^{\text {a }}$ Chuan Wang ${ }^{\mathrm{a}, \mathrm{b}, *}$
}

${ }^{a}$ Hefei National Laboratory for Physical Science at the Microscale, Department of Chemistry, Center for Excellence in Molecular Synthesis, University of Science and Technology of China, 96 Jinzhai Road, Hefei, Anhui 230026 (P. R. China).

${ }^{b}$ Center for Excellence in Molecular Synthesis of CAS, 96 Jinzhai Road, Hefei, Anhui 230026 (P. R. China).

\section{Table of Contents}

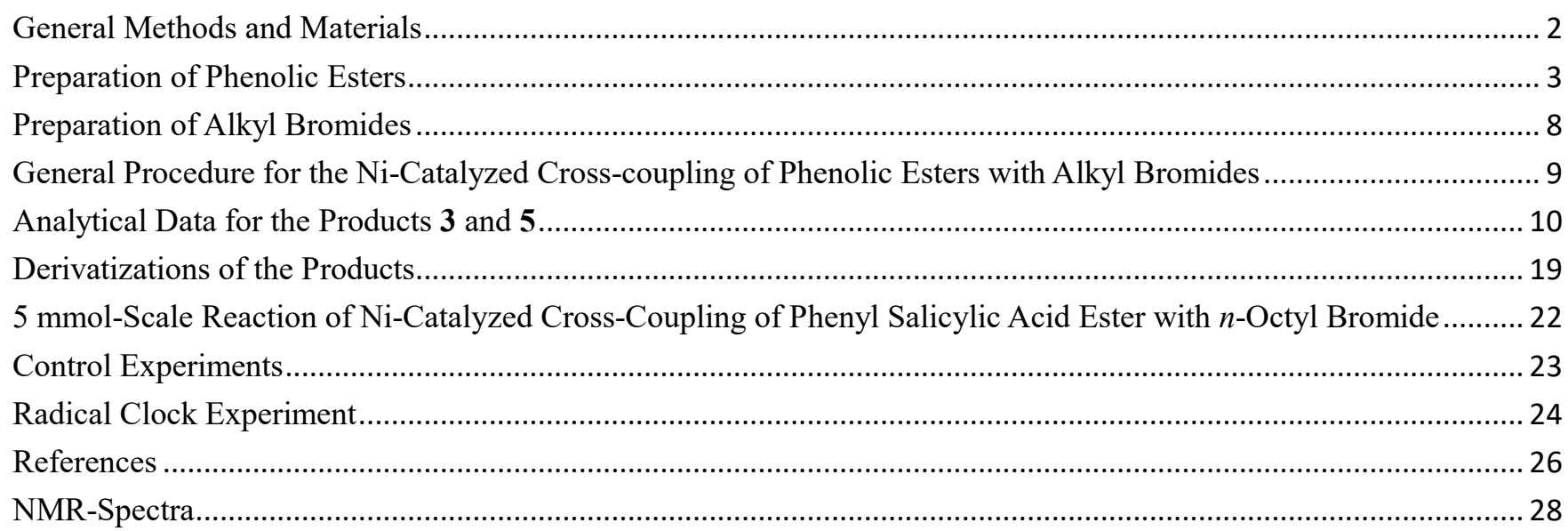




\section{General Methods and Materials}

${ }^{1} \mathrm{H}$ NMR and ${ }^{13} \mathrm{C}$ NMR spectra were recorded on a Bruker Advance 400M or 500M NMR spectrometers at ambient temperature in $\mathrm{CDCl}_{3}$ and DMSO- $\mathrm{d}_{6}$ at 400 and $101 \mathrm{MHz}$, or 500 and $126 \mathrm{MHz}$. The chemical shifts are given in ppm relative to tetramethylsilane $\left[{ }^{1} \mathrm{H}:\left(\mathrm{SiMe}_{4}\right)=0.00 \mathrm{ppm}\right]$ as an internal standard or relative to the resonance of the solvent $\left[{ }^{1} \mathrm{H}: \delta\left(\mathrm{CDCl}_{3}\right)=\right.$ 7.26, $\left.{ }^{13} \mathrm{C}: \delta\left(\mathrm{CDCl}_{3}\right)=77.16 \mathrm{ppm} ;{ }^{1} \mathrm{H}: \delta\left(\mathrm{DMSO}_{6} \mathrm{~d}_{6}\right)=2.50,{ }^{13} \mathrm{C}: \delta\left(\mathrm{DMSO}_{-} \mathrm{d}_{6}\right)=39.52 \mathrm{ppm}\right]$. Multiplicities were given as: $\mathrm{s}$ (singlet); d (doublet); t (triplet); q (quartet); dd (doublet of doublets); dt (doublet of triplets); m (multiplets), etc. Coupling constants are reported as $J$ values in Hz. High resolution mass spectral analysis (HRMS) was performed on Waters XEVO G2 Q-TOF using ESI technique. Flash chromatography was performed using 200-300 mesh silica gel with the indicated solvent system.

Unless otherwise noted, all the reagents and starting materials were purchased from commercial vendors and used without further purification. 


\section{Preparation of Phenolic Esters}<smiles>O=C(Oc1ccccc1)c1ccccc1O</smiles>

1a<smiles>COc1ccc(C(=O)Oc2ccccc2)c(O)c1</smiles>

$1 e$<smiles>Cc1cccc(C(=O)Oc2ccccc2)c1O</smiles>

$1 \mathrm{~b}$

$1 f$<smiles>Cc1ccc(C(=O)Oc2ccccc2)c(O)c1</smiles>

$1 c$<smiles>O=C(Oc1ccccc1)c1ccc(F)cc1O</smiles>

$1 \mathrm{~g}$<smiles>Cc1ccc(O)c(C(=O)Oc2ccccc2)c1</smiles>

$1 d$<smiles>O=C(Oc1ccccc1)c1ccc(C(F)(F)F)cc1O</smiles>

$1 \mathrm{~h}$<smiles>O=C(Oc1ccccc1)c1ccc(O)c(C(=O)Oc2ccccc2)c1</smiles>

$1 \mathrm{i}$<smiles>O=C(Oc1ccccc1)c1cc2ccccc2cc1O</smiles>

$1 \mathrm{j}$<smiles>O=C(Oc1ccccc1)c1cc2ccccc2c(-c2c(C(=O)Oc3ccccc3)cc3ccccc3c2O)c1O</smiles>

$1 \mathrm{k}$<smiles></smiles>

$4 a$<smiles>COc1ccc(NC(=O)c2ccccc2)c(C(=O)Oc2ccccc2)c1</smiles>

4b<smiles>Nc1ccc(F)cc1C(=O)Oc1ccccc1</smiles>

$4 \mathrm{c}$<smiles>Nc1cc(C(F)(F)F)ccc1C(=O)Oc1ccccc1</smiles>

$4 d$

Compounds $\mathbf{1 b} \sim \mathbf{1 h}{ }^{1}$ are known and their NMR-data are consistent with these reported in the literature.

\section{Preparation of Phenolic Ester 1i}<smiles>O=C(O)c1ccc(O)c(C(=O)O)c1</smiles><smiles>Oc1ccccc1</smiles>
toluene, reflux, overnight<smiles>O=C(Oc1ccccc1)c1ccc(O)c(C(=O)Oc2ccccc2)c1</smiles>

$1 \mathbf{i}$

4-Hydroxyisophthalic acid (1.82 g, $10 \mathrm{mmol}, 1$ equiv) and phenol (9.41 g, $100 \mathrm{mmol}, 10$ equiv) were dissolved in $20 \mathrm{~mL}$ of toluene in a $100 \mathrm{~mL}$ round bottle. Phosphorus oxychloride $(1.23 \mathrm{~g}, 0.75 \mathrm{~mL}, 8 \mathrm{mmol}, 0.8$ equiv) was added into the solution via syringe dropwise. After the reaction mixture was stirred under reflux (with oil bath at $110{ }^{\circ} \mathrm{C}$ ) overnight, the mixture was allowed to cool to room temperature, transferred to a separatory funnel, and quenched with saturated aqueous sodium carbonate solution. The aqueous layer was then extracted with ethyl acetate. The organic layers were combined, dried over sodium sulfate, and concentrated in vacuo. The crude product was purified by column chromatography (petroleum ether /ethyl acetate $=20: 1)$ on silica gel to give diphenyl 4-hydroxyisophthalate (1i) as a white solid (1.94 g, $58 \%$ yield). ${ }^{1} \mathrm{H}$ NMR (500 $\left.\mathrm{MHz}, \mathrm{CDCl}_{3}\right) \delta=10.97(\mathrm{~s}, 1 \mathrm{H}), 8.90(\mathrm{~d}, J=2.3 \mathrm{~Hz}, 1 \mathrm{H}), 8.28(\mathrm{dd}, J=8.7,2.3 \mathrm{~Hz}, 1 \mathrm{H}), 7.45-7.34(\mathrm{~m}, 4 \mathrm{H}), 7.29-7.23(\mathrm{~m}, 1 \mathrm{H})$, 7.22-7.14 (m, 5H), $7.08(\mathrm{~d}, J=8.8 \mathrm{~Hz}, 1 \mathrm{H}) \mathrm{ppm} ;{ }^{13} \mathrm{C} \mathrm{NMR}\left(126 \mathrm{MHz}, \mathrm{CDCl}_{3}\right) \delta=167.5,165.1,163.0,149.8,148.8,136.8$, 132.6, 128.7 (2C), 128.5 (2C), 125.7, 125.0, 120.7 (2C), 120.5 (2C), 120.1, 117.4, 110.9 ppm; HRMS (ESI): calcd. for 


\section{Preparation of Phenolic Ester 1j}

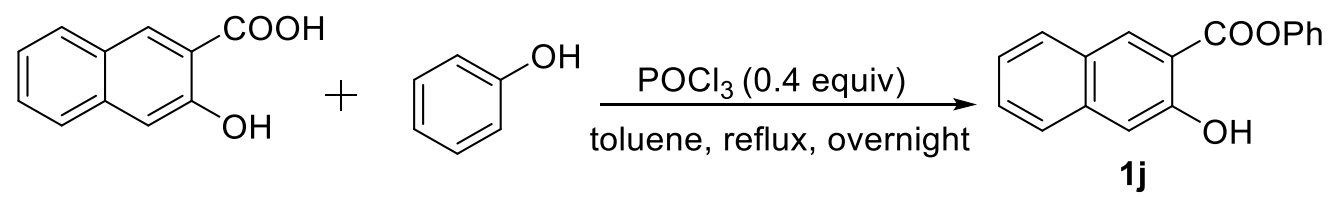

3-Hydroxy-2-naphthoic acid (1.88 g, $10 \mathrm{mmol}, 1$ equiv) and phenol (4.71 g, $50 \mathrm{mmol}, 5$ equiv) were dissolved in $20 \mathrm{~mL}$ of toluene in a $100 \mathrm{~mL}$ round bottle. Phosphorus oxychloride $(0.61 \mathrm{~g}, 0.4 \mathrm{~mL}, 4 \mathrm{mmol}, 0.4$ equiv) was added into the solution via syringe dropwise. After the reaction mixture was stirred under reflux (with oil bath at $110^{\circ} \mathrm{C}$ ) overnight, the mixture was allowed to cool to room temperature, transferred to a separatory funnel, and quenched with saturated aqueous sodium carbonate solution. The aqueous layer was then extracted with ethyl acetate. The organic layers were combined, dried with sodium sulfate, and concentrated in vacuo. The crude product was purified by column chromatography (petroleum ether /ethyl acetate $=10: 1)$ on silica gel to give phenyl 3-hydroxy-2-naphthoate $(\mathbf{1 j})$ as a white solid (1.69 g, $64 \%$ yield). ${ }^{1} \mathrm{H}$ NMR (400 $\left.\mathrm{MHz}, \mathrm{CDCl}_{3}\right) \delta=10.15(\mathrm{~s}, 1 \mathrm{H}), 8.75(\mathrm{~s}, 1 \mathrm{H}), 7.90-7.84(\mathrm{~m}, 1 \mathrm{H}), 7.77-7.70(\mathrm{~m}, 1 \mathrm{H}), 7.58-7.44(\mathrm{~m}, 3 \mathrm{H}), 7.42-7.31(\mathrm{~m}, 3 \mathrm{H})$, 7.77-7.70 (m, 2H) ppm; ${ }^{13} \mathrm{C}$ NMR $\left(101 \mathrm{MHz}, \mathrm{CDCl}_{3}\right) \delta=168.7,156.5,150.1,138.3,133.2,129.8(2 \mathrm{C}), 129.6,129.4,127.1$, 126.6, 126.4, 124.2, 121.7 (2C), 113.6, 112.0 ppm; HRMS (ESI): calcd. for $\mathrm{C}_{17} \mathrm{H}_{13} \mathrm{O}_{3}[\mathrm{M}+\mathrm{H}]^{+}: 265.0859$ found: 265.0866.

\section{Preparation of Phenolic Ester 1k}

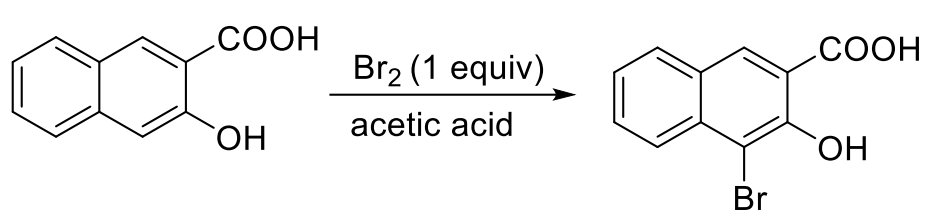

S-1<smiles></smiles>

S-2

$\mathrm{NiBr}_{2}$ diglyme (15 mol \%), DMA (0.4 M)

2,2'-Bipyridine (15 mol \%), Zn (4 equiv)<smiles>O=C(Oc1ccccc1)c1cc2ccccc2c(-c2c(O)c(C(=O)Oc3ccccc3)cc3ccccc23)c1O</smiles>

$1 \mathrm{k}$

Step1: 3-Hydroxynaphthalene-2-carboxylic acid (3.0 g, $15.9 \mathrm{mmol}, 1$ equiv) was suspended in acetic acid (40 $\mathrm{mL})$, and with vigorous stirring a solution of bromine $(817 \mu \mathrm{L}, 15.9 \mathrm{mmol}, 1$ equiv) in acetic acid $(10 \mathrm{~mL})$ was added drop wise within 30 minutes. After stirring at room temperature for 1 hour, the reaction mixture was filtered and washed with water. Evaporation of the volatiles afforded 4-bromo-3-hydroxynaphthalene-2-carboxylic acid (S-1) as a yellow solid $\left(3.85 \mathrm{~g}, 90 \%\right.$ yield); ${ }^{1} \mathrm{H}$ NMR (400 MHz, DMSO- $\left.d_{6}\right) \delta=8.65(\mathrm{~s}, 1 \mathrm{H}), 8.13-8.01(\mathrm{~m}, 2 \mathrm{H}), 7.78-7.67(\mathrm{~m}, 1 \mathrm{H}), 7.54-7.43(\mathrm{~m}, 1 \mathrm{H}) \mathrm{ppm} ;{ }^{13} \mathrm{C}$ NMR $(101$ MHz, DMSO- $\left.d_{6}\right) \delta=172.0,153.8,135.5,132.8,131.2,130.6,127.6,125.2,125.0,115.7,105.9$ ppm; HRMS (ESI): calcd. for $\mathrm{C}_{11} \mathrm{H}_{8} \mathrm{BrO}_{3}[\mathrm{M}+\mathrm{H}]^{+}: 266.9651$ found: 266.9654.

Step2: 4-Bromo-3-hydroxynaphthalene-2-carboxylic acid (S-1) (3.85 g, $14.4 \mathrm{mmol}, 1$ equiv) and phenol (6.78 g, $72 \mathrm{mmol}, 5$ equiv) were were dissolved in $30 \mathrm{~mL}$ of toluene in a $100 \mathrm{~mL}$ round bottle. Phosphorus oxychloride $(0.88 \mathrm{~g}, 0.53 \mathrm{~mL}, 5.76$ mmol, 0.4 equiv) was added into the solution via syringe dropwise. After the reaction mixture was stirred under reflux (with oil bath at $110{ }^{\circ} \mathrm{C}$ ) overnight, the mixture was allowed to cool to room temperature, transferred to a separatory funnel, and 
quenched with saturated aqueous sodium carbonate solution. The aqueous layer was then extracted with ethyl acetate. The organic layers were combined, dried with sodium sulfate, and concentrated in vacuo. The crude product was purified by column chromatography (petroleum ether /ethyl acetate $=20: 1$ ) on silica gel to give phenyl 4-bromo-3-hydroxy-2-naphthoate (S-2) as a yellow solid $\left(3.76 \mathrm{~g}, 76 \%\right.$ yield). ${ }^{1} \mathrm{H}$ NMR $\left(400 \mathrm{MHz}, \mathrm{CDCl}_{3}\right) \delta=10.96(\mathrm{~s}, 1 \mathrm{H}), 8.74(\mathrm{~s}, 1 \mathrm{H}), 8.21(\mathrm{~d}, J=8.7,1 \mathrm{H})$, $7.88(\mathrm{~d}, J=8.2 \mathrm{~Hz}, 1 \mathrm{H}), 7.75-7.62(\mathrm{~m}, 1 \mathrm{H}), 7.54-7.45(\mathrm{~m}, 2 \mathrm{H}), 7.47-7.41(\mathrm{~m}, 1 \mathrm{H}), 7.40-7.31(\mathrm{~m}, 1 \mathrm{H}), 7.31-7.24(\mathrm{~m}, 2 \mathrm{H}) \mathrm{ppm}$; ${ }^{13} \mathrm{C}$ NMR $\left(101 \mathrm{MHz}, \mathrm{CDCl}_{3}\right) \delta=168.4,153.2,150.0,136.5,132.6,131.0,129.9,129.8(2 \mathrm{C}), 127.5,126.7,126.0,124.9,121.5$ (2C), 113.7, 107.4 ppm; HRMS (ESI): calcd. for $\mathrm{C}_{17} \mathrm{H}_{11} \mathrm{BrNaO}_{3}[\mathrm{M}+\mathrm{Na}]^{+}$: 364.9784 found: 364.9787.

\section{Step3:}

A sealed test tube charged with 2,2'-bipyridine (14.0 mg, $0.03 \mathrm{mmol}, 15 \mathrm{~mol} \%)$, 4-bromo-3-hydroxy-2-naphthoate (S-2) (205.9 mg, $0.6 \mathrm{mmol}$ ) and a stir bar was evacuated and filled with nitrogen (three cycles), and then $\mathrm{NiBr}_{2} \bullet$ diglyme (32.0 $\mathrm{mg}$, $0.03 \mathrm{mmol}, 15 \mathrm{~mol} \%$ ) and Zn-powder (150.0 mg, $0.8 \mathrm{mmol}, 4.0$ equiv) were added in the glovebox. Subsequently, DMA (1.2 $\mathrm{mL}$ ) was added to the mixture under nitrogen atmosphere. Then the reaction mixture was heated to $40{ }^{\circ} \mathrm{C}$ with oil bath and stirred at this temperature for $10 \mathrm{~h}$. The mixture was then filtered through a pad of Celite and concentrated under reduced pressure. The residue was purified through column chromatography on silica gel (petroleum ether/ethyl acetate $=10: 1$ ) to afford diphenyl 2,2'-dihydroxy-[1,1'-binaphthalene]-3,3'-dicarboxylate (1k) as a yellow solid (1.84 g, $32 \%$ yield); ${ }^{1} \mathrm{H}$ NMR $\left(400 \mathrm{MHz}, \mathrm{CDCl}_{3}\right) \delta=10.94(\mathrm{~s}, 1 \mathrm{H}), 10.14(\mathrm{~s}, 1 \mathrm{H}), 8.70(\mathrm{~d}, J=8.1 \mathrm{~Hz}, 2 \mathrm{H}), 8.18(\mathrm{~d}, J=8.6 \mathrm{~Hz}, 1 \mathrm{H}), 7.83(\mathrm{dd}, J=8.3,3.4$ $\mathrm{Hz}, 2 \mathrm{H}), 7.71-7.60(\mathrm{~m}, 2 \mathrm{H}), 7.54-7.50(\mathrm{~m}, 1 \mathrm{H}), 7.49(\mathrm{~d}, J=2.7 \mathrm{~Hz}, 1 \mathrm{H}), 7.47(\mathrm{~d}, J=3.2 \mathrm{~Hz}, 2 \mathrm{H}), 7.45(\mathrm{~d}, J=3.1 \mathrm{~Hz}, 1 \mathrm{H})$, 7.43-7.38 (m, 1H), 7.36-7.33 (m, 3H), 7.33-7.31 (m, 1H), 7.28-7.26 (m, 1H), 7.26-7.22 (m, 2H) ppm; ${ }^{13} \mathrm{C}$ NMR (126 MHz, $\left.\mathrm{CDCl}_{3}\right) \delta=168.7,168.4,156.5,153.2,150.2,150.0,138.3,136.5,133.2,132.6,131.0,129.9,129.84$ (2C), 129.75 (2C), 129.6, 129.4, 127.6, 127.2, 126.8, 126.6, 126.4, 126.0, 124.9, 124.2, 121.7 (2C), 121.6 (2C), 113.7, 113.6, 112.0, 107.4 ppm; HRMS (ESI): calcd. for $\mathrm{C}_{34} \mathrm{H}_{23} \mathrm{O}_{6}[\mathrm{M}+\mathrm{H}]^{+}: 527.1489$ found: 527.1493 .

\section{Preparation of Salicylate Esters 4a, 4c and 4d}
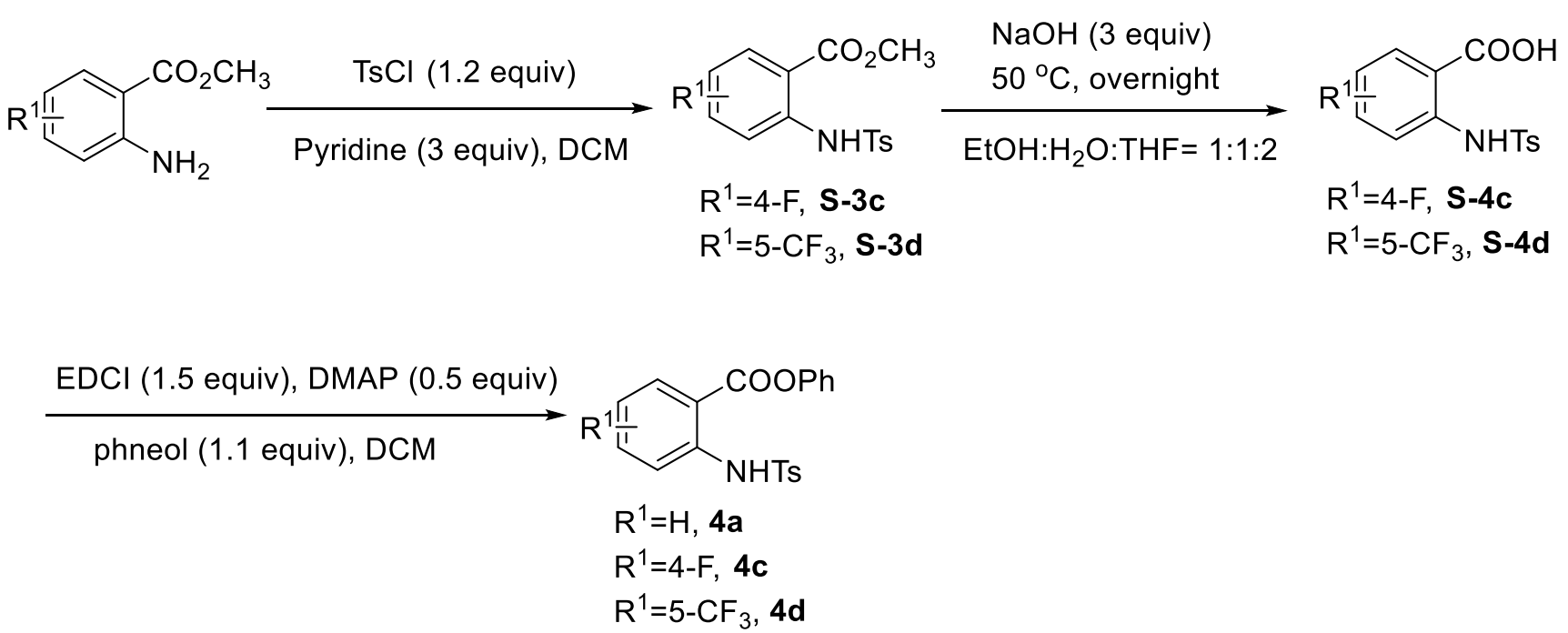

Step 1: To a solution of methyl 2-aminobenzoates (10.0 mmol, 1 equiv) in $20 \mathrm{~mL}$ of DCM under $\mathrm{N}_{2}$ atmosphere, were added pyridine ( $2.4 \mathrm{~mL}, 30 \mathrm{mmol}, 3$ equiv) and tosyl chloride ( $2.3 \mathrm{~g}, 12 \mathrm{mmol}, 1.2$ equiv). After the mixture was stirred at room temperature for $1 \mathrm{~h}$, the reaction was quenched by addition of water. The mixture was extracted with EtOAc, and the combined organic layers were washed with brine and dried over $\mathrm{Na}_{2} \mathrm{SO}_{4}$. After removal of the solvent, the residue was purified by column chromatography on silica gel (petroleum ether / EtOAc=10:1) to give $\mathbf{S - 3 c}$ and $\mathbf{S - 3 d}$.

Step2: S-3 (8 mmol, 1 equiv) and $\mathrm{NaOH}\left(0.96 \mathrm{~g}, 24 \mathrm{mmol}, 3\right.$ equiv) were dissolved in $\mathrm{H}_{2} \mathrm{O}$ (10 mL), EtOH (10 mL) and THF 
$(20 \mathrm{~mL})$. The reaction mixture was stirred for overnight with oil bath at $50^{\circ} \mathrm{C}$. The organic solvent was then removed under vacuum. The residue was extracted with ethyl acetate, The water layer was acidified with concentrated $\mathrm{HCl}$ until $\mathrm{pH}=2$ and extracted with ethyl acetate. The combined organic layers were concentrated under vacuum to afford $\mathbf{S}-\mathbf{4} \mathbf{c}$ and $\mathbf{S}-\mathbf{4 d}$.

Step3: S-4 ( 8 mmol, 1 equiv), ${ }^{\text {a }}$ EDCI ( $2.3 \mathrm{~g}, 24 \mathrm{mmol}, 1.5$ equiv), DMAP (488.8 mg, 4 mmol, 0.5 equiv) and phenol (828.2 $\mathrm{mg}, 8.8 \mathrm{mmol}, 1.1$ equiv) were dissolved in DCM $(20 \mathrm{~mL})$. The reaction mixture was stirred for overnight at room temperature. The reaction was quenched by addition of water. The mixture was extracted with DCM and combined organic layers were washed with brine and dried over $\mathrm{Na}_{2} \mathrm{SO}_{4}$, After removal of the solvent, the residue was purified by column chromatography on silica gel (petroleum ether / EtOAc=10:1) to give $\mathbf{4 a}, \mathbf{4 c}, \mathbf{4 d}$.

${ }^{a} \mathbf{S}-\mathbf{4 a}$ was prepared according to the method reported in the literature. ${ }^{2,3}$

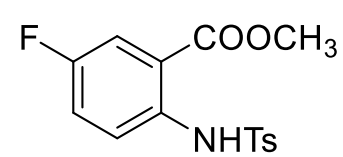

S-3c

Methyl 5-fluoro-2-((4-methylphenyl)sulfonamido)benzoate (S-3c) was isolated through column chromatography on silica gel with petroleum ether to the mixture eluent of petroleum ether and ethyl acetate $(10: 1)$ as a white solid $\left(2.70 \mathrm{~g}, 84 \%\right.$ yield). ${ }^{1} \mathrm{H}$ NMR $\left(400 \mathrm{MHz}, \mathrm{CDCl}_{3}\right) \delta=10.29(\mathrm{~s}, 1 \mathrm{H})$, 7.73-7.65 (m, 3H), $7.57(\mathrm{dd}, J=9.0,3.1 \mathrm{~Hz}, 1 \mathrm{H}), 7.23-7.17(\mathrm{~m}, 3 \mathrm{H}), 3.86(\mathrm{~s}, 3 \mathrm{H}), 2.37$ (s, 3H) ppm; ${ }^{13} \mathrm{C}$ NMR $\left(101 \mathrm{MHz}, \mathrm{CDCl}_{3}\right) \delta=167.1(\mathrm{~d}, J=2.6 \mathrm{~Hz}), 157.9(\mathrm{~d}, J=244.5 \mathrm{~Hz}), 144.0,136.6(\mathrm{~d}, J=$ $2.7 \mathrm{~Hz}), 136.0,129.6(2 \mathrm{C}), 127.2(2 \mathrm{C}), 121.8,121.7(\mathrm{~d}, J=14.5 \mathrm{~Hz}), 117.7$ (d, $J=7.2 \mathrm{~Hz}), 117.2(\mathrm{~d}, J=24.3 \mathrm{~Hz}), 52.6,21.5$ ppm; ${ }^{19} \mathrm{~F}$ NMR (376 MHz, $\left.\mathrm{CDCl}_{3}\right) \delta=-118.00--118.86$ (m, $\left.1 \mathrm{~F}\right)$ ppm; HRMS (ESI): calcd. for $\mathrm{C}_{15} \mathrm{H}_{14} \mathrm{FNO}_{4} \mathrm{SNa}[\mathrm{M}+\mathrm{Na}]^{+}$: 346.0520 found: 346.0520 .

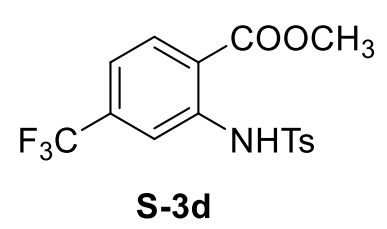

Methyl 2-((4-methylphenyl)sulfonamido)-4-(trifluoromethyl)benzoate (S-3d) was isolated through column chromatography on silica gel with petroleum ether to the mixture eluent of petroleum ether and ethyl acetate (10:1) as a white solid (1.80 g, $82 \%$ yield). ${ }^{1} \mathrm{H}$ NMR (400 MHz, $\left.\mathrm{CDCl}_{3}\right) \delta=10.71(\mathrm{~s}, 1 \mathrm{H}), 8.03(\mathrm{dd}, J=8.3,0.9 \mathrm{~Hz}, 1 \mathrm{H}), 7.98(\mathrm{~d}, J=1.6 \mathrm{~Hz}, 1 \mathrm{H}), 7.77(\mathrm{~d}, J=8.4$ $\mathrm{Hz}, 2 \mathrm{H}), 7.26-7.23(\mathrm{~m}, 3 \mathrm{H}), 3.93(\mathrm{~s}, 3 \mathrm{H}), 2.38(\mathrm{~s}, 3 \mathrm{H}) \mathrm{ppm} ;{ }^{13} \mathrm{C} \mathrm{NMR}\left(101 \mathrm{MHz}, \mathrm{CDCl}_{3}\right) \delta=$ $167.4,144.4,141.0,135.9,135.8(\mathrm{q}, J=32.3 \mathrm{~Hz}), 131.9,129.8(2 \mathrm{C}), 127.4(2 \mathrm{C}), 123.0(\mathrm{q}, J=273.3 \mathrm{~Hz}), 119.0(\mathrm{q}, J=$ $3.7 \mathrm{~Hz}), 118.0,115.5$ (q, $J=4.0 \mathrm{~Hz}), 52.9,21.6 \mathrm{ppm} ;{ }^{19} \mathrm{~F} \mathrm{NMR}\left(376 \mathrm{MHz}, \mathrm{CDCl}_{3}\right) \delta=-63.72$ (s, 3F) ppm; HRMS (ESI): calcd. for $\mathrm{C}_{16} \mathrm{H}_{14} \mathrm{~F}_{3} \mathrm{NO}_{4} \mathrm{SNa}[\mathrm{M}+\mathrm{Na}]^{+}: 396.0488$ found: 396.0490 .<smiles>Nc1ccc(F)cc1C(=O)O</smiles>

S-4c

5-Fluoro-2-((4-methylphenyl)sulfonamido)benzoic acid (S-4c) was isolated through column chromatography on silica gel with petroleum ether to the mixture eluent of petroleum ether and ethyl acetate (2:1) as a white solid (2.42 g, $98 \%$ yield). ${ }^{1} \mathrm{H}$ NMR (400 MHz, DMSO- $\left.d_{6}\right) \delta=7.67-7.56(\mathrm{~m}$, $3 \mathrm{H}), 7.46$ (dd, $J=9.1,4.8 \mathrm{~Hz}, 1 \mathrm{H}), 7.36-7.19$ (m, 3H), 2.31 (s, 3H) ppm; ${ }^{13} \mathrm{C}$ NMR (126 MHz, DMSO$\left.d_{6}\right) \delta=168.9,157.0(\mathrm{~d}, J=244.5 \mathrm{~Hz}), 144.6,136.5(\mathrm{~d}, J=3.3 \mathrm{~Hz}), 136.0,130.4(2 \mathrm{C}), 127.3(2 \mathrm{C})$, $122.0(\mathrm{~d}, J=2.9 \mathrm{~Hz}), 121.9(\mathrm{~d}, J=11.9 \mathrm{~Hz}), 119.8(\mathrm{~d}, J=7.1 \mathrm{~Hz}), 117.8(\mathrm{~d}, J=24.0 \mathrm{~Hz}), 21.4 \mathrm{ppm} ;{ }^{19} \mathrm{~F}$ NMR $(471 \mathrm{MHz}$, DMSO- $\left.d_{6}\right) \delta=-118.01$ - $-118.46(\mathrm{~m}, 1 \mathrm{~F})$; HRMS (ESI): calcd. for $\mathrm{C}_{14} \mathrm{H}_{12} \mathrm{FNO}_{4} \mathrm{SNa}[\mathrm{M}+\mathrm{Na}]^{+}: 332.0363$ found: 330.0367 .

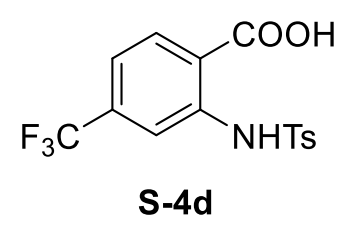

2-((4-Methylphenyl)sulfonamido)-4-(trifluoromethyl)benzoic acid (S-4d) was isolated through column chromatography on silica gel with petroleum ether to the mixture eluent of petroleum ether and ethyl acetate (2:1) as a white solid (2.81 g, $98 \%$ yield). ${ }^{1} \mathrm{H}$ NMR (400 MHz, DMSO- $\left.d_{6}\right) \delta=8.08$ $(\mathrm{d}, J=8.1 \mathrm{~Hz}, 1 \mathrm{H}), 7.76-7.63(\mathrm{~m}, 3 \mathrm{H}), 7.33(\mathrm{~d}, J=8.0 \mathrm{~Hz}, 2 \mathrm{H}), 7.25(\mathrm{~d}, J=8.1 \mathrm{~Hz}, 1 \mathrm{H}), 2.31(\mathrm{~s}$, $3 \mathrm{H}) \mathrm{ppm} ;{ }^{13} \mathrm{C}$ NMR $\left(101 \mathrm{MHz}, \mathrm{DMSO}-d_{6}\right) \delta=168.7,143.5,143.4,137.9,132.8,132.3$ (q, $J=31.8$ $\mathrm{Hz}), 130.1$ (2C), 126.9 (2C), 124.5, 124.1 (q, $J=272.9 \mathrm{~Hz}), 117.7$ (q, $J=3.9 \mathrm{~Hz}), 114.2$ (q, $J=4.0 \mathrm{~Hz}), 21.3 ;{ }^{19} \mathrm{~F}$ NMR $(376$ $\left.\mathrm{MHz}, \mathrm{DMSO}-d_{6}\right) \delta=-61.98(\mathrm{~s}, 3 \mathrm{~F}) \mathrm{ppm}$. HRMS (ESI): calcd. for $\mathrm{C}_{15} \mathrm{H}_{12} \mathrm{~F}_{3} \mathrm{NO}_{4} \mathrm{SNa}[\mathrm{M}+\mathrm{Na}]^{+}: 382.0331$ found: 382.0341. 
1-(2-Hydroxyphenyl)nonan-1-one (4a) was isolated through column chromatography on silica gel with petroleum ether to the mixture eluent of petroleum ether and ethyl acetate (10:1) as a white solid $(2.20 \mathrm{~g}$, $60 \%$ yield). ${ }^{1} \mathrm{H}$ NMR $\left(400 \mathrm{MHz}, \mathrm{CDCl}_{3}\right) \delta=10.44(\mathrm{~s}, 1 \mathrm{H}), 8.16(\mathrm{dd}, J=8.0,1.7 \mathrm{~Hz}, 1 \mathrm{H}), 7.79-7.70(\mathrm{~m}$, $3 \mathrm{H}), 7.57-7.47(\mathrm{~m}, 1 \mathrm{H}), 7.46-7.40(\mathrm{~m}, 2 \mathrm{H}), 7.34-7.25(\mathrm{~m}, 1 \mathrm{H}), 7.22(\mathrm{~d}, J=8.1 \mathrm{~Hz}, 2 \mathrm{H}), 7.16-7.06(\mathrm{~m}$, $3 \mathrm{H}), 2.36(\mathrm{~s}, 3 \mathrm{H}) \mathrm{ppm} ;{ }^{13} \mathrm{C} \mathrm{NMR}\left(101 \mathrm{MHz}, \mathrm{CDCl}_{3}\right) \delta=166.8,150.1,144.1,141.2,136.4,135.3,131.7$, 129.74 (2C), 129.69 (2C), 127.4 (2C), 126.5, 123.1, 121.6 (2C), 119.2, 115.1, 21.6 ppm; HRMS (ESI): calcd. for $\mathrm{C}_{20} \mathrm{H}_{17} \mathrm{NO}_{4} \mathrm{SNa}[\mathrm{M}+\mathrm{Na}]^{+}: 390.0770$ found: 390.0779 .

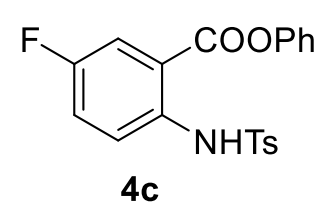

Phenyl 5-fluoro-2-((4-methylphenyl)sulfonamido)benzoate (4c) was isolated through column chromatography on silica gel with petroleum ether to the mixture eluent of petroleum ether and ethyl acetate $(10: 1)$ as a white solid $\left(2.64 \mathrm{~g}, 86 \%\right.$ yield). ${ }^{1} \mathrm{H}$ NMR $\left(400 \mathrm{MHz}, \mathrm{CDCl}_{3}\right) \delta=10.09(\mathrm{~s}, 1 \mathrm{H})$, 7.86-7.76 (m, 2H), 7.72-7.65 (m, 2H), 7.49-7.42 (m, 2H), 7.35-7.28 (m, 2H), 7.24 (d, J=8.1 Hz, 2H), 7.13-7.04 (m, 2H), 2.39 (s, 3H) ppm; ${ }^{13} \mathrm{C} \mathrm{NMR}\left(101 \mathrm{MHz}, \mathrm{CDCl}_{3}\right) \delta=165.5(\mathrm{~d}, J=2.6 \mathrm{~Hz}), 158.0(\mathrm{~d}$, $J=244.9 \mathrm{~Hz}), 149.9,144.1,137.3$ (d, $J=2.7 \mathrm{~Hz}), 136.1,129.7$ (4C), $127.3(2 \mathrm{C}), 126.6,122.5$ (d, $J=22.5 \mathrm{~Hz}), 122.1(\mathrm{~d}, J=$ $7.6 \mathrm{~Hz}), 121.4(2 \mathrm{C}), 117.6(\mathrm{~d}, J=24.5 \mathrm{~Hz}), 117.0(\mathrm{~d}, J=7.3 \mathrm{~Hz}), 21.6 \mathrm{ppm} ;{ }^{19} \mathrm{~F}$ NMR $\left(376 \mathrm{MHz}, \mathrm{CDCl}_{3}\right) \delta=-114.76--$ 125.43 (m, 1F) ppm; HRMS (ESI): calcd. for $\mathrm{C}_{20} \mathrm{H}_{16} \mathrm{FNO}_{4} \mathrm{SNa}$ [M+Na] $]^{+}: 408.0676$ found: 408.0682

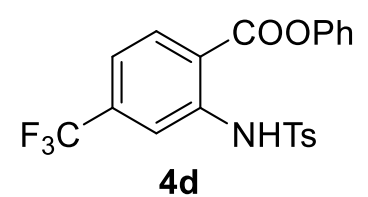

Phenyl 2-((4-methylphenyl)sulfonamido)-4-(trifluoromethyl)benzoate (4d) was isolated through column chromatography on silica gel with petroleum ether to the mixture eluent of petroleum ether and ethyl acetate (10:1) as a white solid (2.34 g, $68 \%$ yield). ${ }^{1} \mathrm{H}$ NMR $\left(400 \mathrm{MHz}, \mathrm{CDCl}_{3}\right) \delta=10.51$ $(\mathrm{s}, 1 \mathrm{H}), 8.33-8.24(\mathrm{~m}, 1 \mathrm{H}), 8.06(\mathrm{~s}, 1 \mathrm{H}), 7.81-7.74(\mathrm{~m}, 2 \mathrm{H}), 7.49-7.42(\mathrm{~m}, 2 \mathrm{H}), 7.38-7.30(\mathrm{~m}, 2 \mathrm{H})$, 7.29-7.22 (m, 2H), 7.18- 7.07 (m, 2H), 2.39 (s, 3H) ppm; $\left.{ }^{13} \mathrm{C} \mathrm{NMR} \mathrm{(101} \mathrm{MHz,} \mathrm{CDCl}_{3}\right) \delta=165.8$, 149.8, 144.6, 141.7, 136.4 (q, $J=33.2 \mathrm{~Hz}), 135.9,132.4,129.9$ (2C), 129.8 (2C), $127.4(2 \mathrm{C}), 126.8,123.0$ (q, $J=276.7 \mathrm{~Hz})$, $121.4(2 \mathrm{C}), 119.1$ (q, $J=3.6 \mathrm{~Hz}), 117.3,115.6$ (q, $J=4.0 \mathrm{~Hz}), 21.6 \mathrm{ppm} ;{ }^{19} \mathrm{~F}$ NMR $\left(376 \mathrm{MHz}, \mathrm{CDCl}_{3}\right) \delta=-63.75(\mathrm{~s}, 3 \mathrm{~F}) \mathrm{ppm}$; HRMS (ESI): calcd. for $\mathrm{C}_{21} \mathrm{H}_{16} \mathrm{~F}_{3} \mathrm{NO}_{4} \mathrm{SNa}$ [M+Na] $]^{+}: 458.0644$ found: 458.0649

\section{Preparation of Salicylate Ester $4 b$}

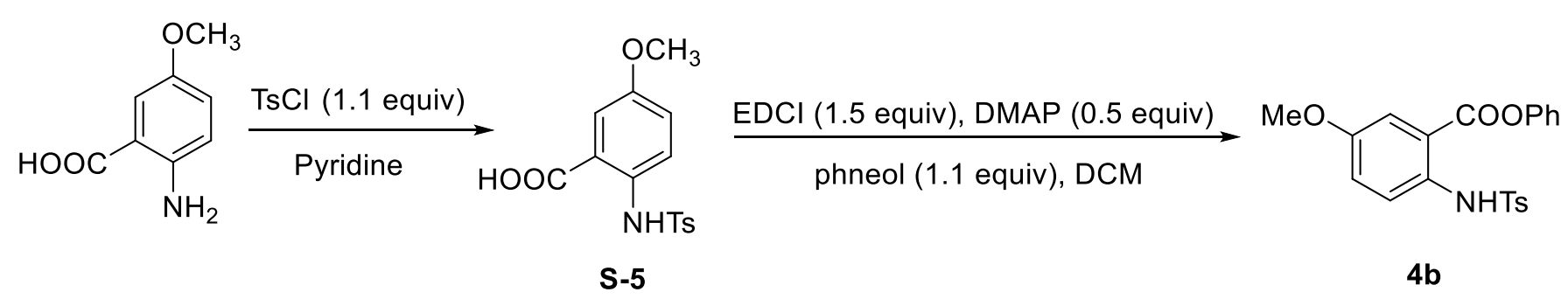

To a solution of a 2-amino-5-methoxybenzoic acid (1.67 g, $10.0 \mathrm{mmol}, 1$ equiv) in dry pyridine $(20 \mathrm{~mL})$, tosyl chloride (2.09 $\mathrm{g}, 11 \mathrm{mmol}, 1.1$ equiv) was added at $0{ }^{\circ} \mathrm{C}$ under nitrogen atmosphere. After $10 \mathrm{~h}$, the reaction was quenched with ice water, and the aqueous layer was extracted with DCM. The organic layers were combined, washed with $\mathrm{HCl} 10 \%$ (5 mL), brine (10 $\mathrm{mL}$ ), dried over $\mathrm{MgSO}_{4}$, filtered and concentrated under reduced pressure. The residue was purified by column chromatography on silica gel (petroleum ether / $\mathrm{EtOAc}=1: 1$ ) to give $\mathbf{S - 5}$.<smiles>COc1ccc(NC(=O)c2ccccc2)c(C(=O)O)c1</smiles>

S-5

5-Methoxy-2-((4-methylphenyl)sulfonamido)benzoic acid (S-5) was isolated through column chromatography on silica gel with petroleum ether to the mixture eluent of petroleum ether and ethyl acetate (1:1) as a white solid ( $2.60 \mathrm{~g}, 81 \%$ yield). ${ }^{1} \mathrm{H}$ NMR $\left(500 \mathrm{MHz}, \mathrm{DMSO}-d_{6}\right) \delta=10.53$ (s, 1H), 7.59 (d, $J=8.4 \mathrm{~Hz}, 2 \mathrm{H}), 7.47$ (d, $J=9.0 \mathrm{~Hz}, 1 \mathrm{H}), 7.36-7.30$ (m, 3H), 7.18 (dd, $J=9.0,3.2$ 
$\mathrm{Hz}, 1 \mathrm{H}), 3.72$ (s, 3H), 2.33 (s, 3H) ppm; ${ }^{13} \mathrm{C}$ NMR (126 MHz, DMSO-d $\left.)\right) \delta=169.6,155.6,144.3,136.2,133.1,130.3(2 \mathrm{C})$, 127.3 (2C), 122.3, 121.0, 119.8, 115.5, 55.9, 21.4 ppm; HRMS (ESI): calcd. for $\mathrm{C}_{15} \mathrm{H}_{15} \mathrm{NO}_{5} \mathrm{SNa}[\mathrm{M}+\mathrm{Na}]^{+}: 344.0563$ found: 344.0568 .<smiles>COc1ccc(NS)c(C(=O)Oc2ccccc2)c1</smiles>

4b

Phenyl 5-methoxy-2-((4-methylphenyl)sulfonamido)benzoate (4b) was isolated through column chromatography on silica gel with petroleum ether to the mixture eluent of petroleum ether and ethyl acetate (10:1) as a white solid $\left(2.77 \mathrm{~g}, 87 \%\right.$ yield). ${ }^{1} \mathrm{H}$ NMR $\left(400 \mathrm{MHz}, \mathrm{CDCl}_{3}\right) \delta=9.82(\mathrm{~s}$, $1 \mathrm{H}), 7.74(\mathrm{~d}, J=9.1 \mathrm{~Hz}, 1 \mathrm{H}), 7.67-7.61(\mathrm{~m}, 2 \mathrm{H}), 7.59(\mathrm{~d}, J=3.1 \mathrm{~Hz}, 1 \mathrm{H}), 7.48-7.41(\mathrm{~m}, 2 \mathrm{H}), 7.34$ $-7.28(\mathrm{~m}, 1 \mathrm{H}), 7.23-7.19(\mathrm{~m}, 2 \mathrm{H}), 7.14(\mathrm{dd}, J=9.1,3.1 \mathrm{~Hz}, 1 \mathrm{H}), 7.10-7.00(\mathrm{~m}, 2 \mathrm{H}), 3.82(\mathrm{~s}, 3 \mathrm{H})$, $2.38(\mathrm{~s}, 3 \mathrm{H}) \mathrm{ppm} ;{ }^{13} \mathrm{C} \mathrm{NMR}\left(101 \mathrm{MHz}, \mathrm{CDCl}_{3}\right) \delta=166.1,155.6,150.0,143.8,136.2,134.1,129.6(2 \mathrm{C}), 129.5(2 \mathrm{C}), 127.3$ (2C), 126.5, 122.9, 121.7, 121.5 (2C), 117.4, 115.1, 55.7, 21.6 ppm. HRMS (ESI): calcd. for $\mathrm{C}_{21} \mathrm{H}_{19} \mathrm{NO}_{5} \mathrm{SNa}[\mathrm{M}+\mathrm{Na}]^{+}$: 420.0876 found:420.0887.

\section{Preparation of Alkyl Bromides}<smiles>COC(=O)CCCCCBr</smiles>

2a<smiles>CCCCC(CC)CBr</smiles>

$2 e$

2i<smiles>ClCCCCCBr</smiles><smiles>CCCCCCCCBr</smiles>

$2 b$<smiles>BrCC1CCCCC1</smiles>

2c<smiles>BrCCC1CCCCC1</smiles>

2d<smiles>BrCCPc1ccccc1</smiles>

$2 f$<smiles>BrCCCCc1ccccc1</smiles>

$2 \mathrm{~g}$<smiles>FCCCCCBr</smiles>

$2 h$<smiles>COc1ccc(OCCCCCCBr)cc1</smiles>

$2 \mathbf{j}$<smiles>S[O+]CCCCCCBr</smiles>

2k

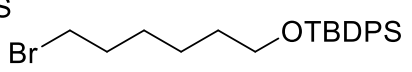

2I<smiles>O=C(CCCCCBr)OC1CCC2(CC1)OCCO2</smiles>

$2 m$<smiles>CC(C)(O)OCCCCCCBr</smiles>

$2 q$<smiles>CCCC(Br)CCc1ccccc1</smiles>

$2 u$<smiles>O=C(OCCCCCBr)c1ccc2ccccc2c1</smiles>

$2 r$<smiles>CCC[In]C1CCC(C)C[C@H]1Br</smiles>

$2 v$<smiles>O=C(CCCCCBr)OCC(F)(F)F</smiles><smiles>O=C(CCCCCBr)Oc1ccccc1</smiles>

20

$2 p$<smiles>BrC1CCCCC1</smiles>

2t

$2 s$<smiles>COc1ccc(CCC(C)Br)cc1</smiles>

$2 x$

Compounds $\mathbf{2 a - i ,} \mathbf{2 p}, \mathbf{2 s}, \mathbf{2 t}$ and $\mathbf{2 w}$ are commercially available. Compounds $\mathbf{2} \mathbf{j}^{4}, \mathbf{2} \mathbf{k}^{5}, \mathbf{2} \mathbf{l}^{6}, \mathbf{2} \mathbf{n}^{7}, \mathbf{2 0}{ }^{7}, \mathbf{2} \mathbf{q}^{7}, \mathbf{2} \mathbf{u}^{8}, \mathbf{2 v}{ }^{7}, \mathbf{2} \mathbf{x}^{9}$ are known and their NMR-data are consistent with these reported in the literature. 


\section{Preparation of Alkyl Bromide 2m and 2r.}

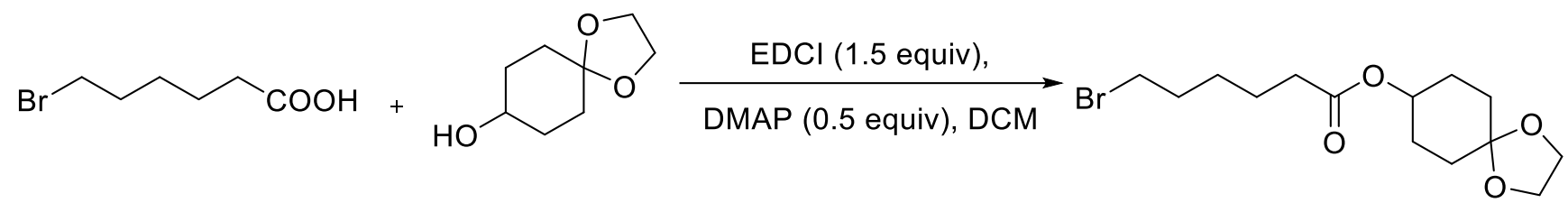

$2 m$

6-Bromohexanoic acid (2.1 g, $11.0 \mathrm{mmol}, 1.1$ equiv), EDCI (2.9 g, $15 \mathrm{mmol}, 1.5$ equiv), DMAP (610.0 mg, $5 \mathrm{mmol}, 0.5$ equiv) and 1,4-dioxa-spiro[4.5]decan-8-ol (1.58 g, $10.0 \mathrm{mmol}, 1.0$ equiv) were dissolved in DCM (20 mL). The reaction mixture was stirred for overnight at room temperature, before it was quenched by addition of water. The mixture was extracted with DCM and combined organic layers were washed with brine and dried over $\mathrm{Na}_{2} \mathrm{SO}_{4}$, After removal of the solvent, the residue was purified by column chromatography on silica gel (petroleum ether / EtOAc $=10: 1$ ) to give 1,4dioxaspiro[4.5]Decan-8-yl 6-bromohexanoate $(\mathbf{2 m})$ as a colorless oil $\left(2.74 \mathrm{~g}, 82 \%\right.$ yield). ${ }^{1} \mathrm{H}$ NMR $\left(500 \mathrm{MHz}, \mathrm{CDCl}_{3}\right) \delta=$ 5.00-4.65 (m, 1H), 4.00-3.82 (m, 4H), $3.41(\mathrm{t}, J=6.8 \mathrm{~Hz}, 2 \mathrm{H}), 2.32(\mathrm{t}, J=7.4 \mathrm{~Hz}, 2 \mathrm{H}), 1.91-1.84(\mathrm{~m}, 4 \mathrm{H}), 1.82-1.71(\mathrm{~m}, 4 \mathrm{H})$, $1.69-1.56$ (m, 4H), 1.52-1.43 (m, 2H) ppm; $\left.{ }^{13} \mathrm{C} \mathrm{NMR} \mathrm{(126} \mathrm{MHz,} \mathrm{CDCl}_{3}\right) \delta=172.8,107.9,69.9,64.29,64.27,34.3,33.5,32.3$, 31.2 (2C), 28.3 (2C), 27.6, 24.1 ppm; HRMS (ESI): calcd. for $\mathrm{C}_{14} \mathrm{H}_{24} \mathrm{BrO}_{4}[\mathrm{M}+\mathrm{H}]^{+}: 335.0852$ found:335.0856.
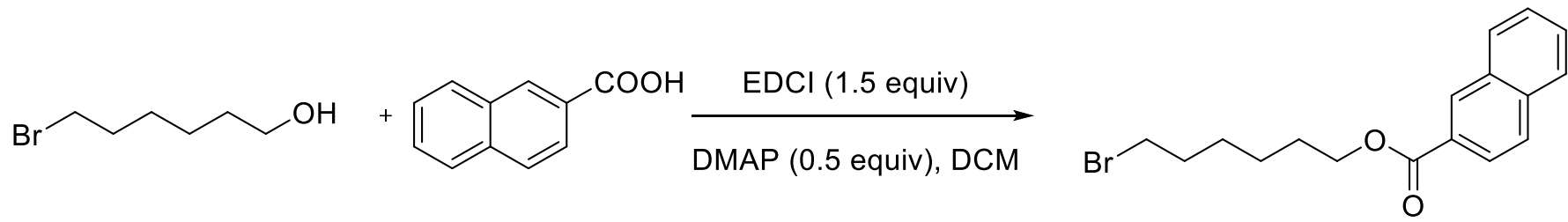

$2 \mathbf{r}$

6-Bromohexan-1-ol (1.8 g, 10.0 mmol, 1.0 equiv), EDCI (2.9 g, 15 mmol, 1.5 equiv), DMAP (610.0 mg, 5 mmol, 0.5 equiv) and 2-naphthoic acid (1.9 g, $11.0 \mathrm{mmol}, 1.1$ equiv) were dissolved in DCM $(20 \mathrm{~mL})$. The reaction mixture was stirred for overnight at room temperature., before it was quenched by addition of water. The mixture was extracted with DCM and combined organic layers were washed with brine and dried over $\mathrm{Na}_{2} \mathrm{SO}_{4}$, After removal of the solvent, the residue was purified by column chromatography on silica gel (petroleum ether / $\mathrm{EtOAc}=10: 1)$ to give 6-bromohexyl 2-naphthoate (2r) as a white solid $\left(2.68 \mathrm{~g}, 80 \%\right.$ yield). ${ }^{1} \mathrm{H}$ NMR $\left(500 \mathrm{MHz}, \mathrm{CDCl}_{3}\right) \delta=8.60(\mathrm{~s}, 1 \mathrm{H}), 8.06(\mathrm{~d}, J=8.5 \mathrm{~Hz}, 1 \mathrm{H}), 7.96(\mathrm{~d}, J=8.1 \mathrm{~Hz}, 1 \mathrm{H})$, $7.88(\mathrm{~d}, J=8.6 \mathrm{~Hz}, 2 \mathrm{H}), 7.63-7.48(\mathrm{~m}, 2 \mathrm{H}), 4.38(\mathrm{t}, J=6.6 \mathrm{~Hz}, 2 \mathrm{H}), 3.42(\mathrm{t}, J=6.8 \mathrm{~Hz}, 2 \mathrm{H}), 1.92-1.82(\mathrm{~m}, 4 \mathrm{H}), 1.60-1.44$ (m, 4H) ppm; ${ }^{13} \mathrm{C}$ NMR $\left(126 \mathrm{MHz}, \mathrm{CDCl}_{3}\right) \delta=166.8,135.5,132.5,131.0,129.4,128.2,128.2,127.8,127.7,126.7,125.3$, 65.0, 33.8, 32.7, 28.7, 27.9, 25.4 ppm; HRMS (ESI): calcd. for $\mathrm{C}_{17} \mathrm{H}_{20} \mathrm{BrO}_{2}[\mathrm{M}+\mathrm{H}]^{+}: 335.0641$ found:335.0635.

\section{General Procedure for the Ni-Catalyzed Cross-coupling of Phenolic Esters with Alkyl Bromides}<smiles></smiles>

$X=0, N T s$

1 or 4<smiles>[R]C([R])Br</smiles>

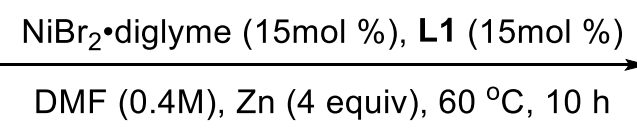

DMF (0.4M), Zn (4 equiv), $60^{\circ} \mathrm{C}, 10 \mathrm{~h}$<smiles>[Y]c1c[14c]([R3])ccc1C(=O)C([R])[R]</smiles>

3 or 5<smiles>c1cnc2c(c1)ccc1cccnc12</smiles>

L1 
A sealed reaction tube charged with the phenolic esters 1 or 4 ( $0.2 \mathrm{mmol}, 1$ equiv), ligand $\mathbf{L 1}$ (5.4 $\mathrm{mg}, 0.03 \mathrm{mmol}, 15 \mathrm{~mol} \%)$ and a stir bar was evacuated and filled with nitrogen (three cycles). In a nitrogen-filled glovebox, $\mathrm{NiBr}_{2} \bullet$ diglyme $(10.6 \mathrm{mg}$, $0.03 \mathrm{mmol}, 15 \mathrm{~mol} \%$ ) and $\mathrm{Zn}$-powder ( $52 \mathrm{mg}, 0.8 \mathrm{mmol}, 4.0$ equiv) were added to the mixture. The reaction tube was then sealed and removed from the glove box. Subsequently, DMF $(0.5 \mathrm{~mL})$ was added to the mixture under nitrogen atmosphere. Then the reaction mixture was heated to $60^{\circ} \mathrm{C}$ with a heating block, before alkyl bormides 2 ( $0.3 \mathrm{mmol}, 1.5$ equiv) ${ }^{\mathrm{a}}$ were added. After stirring at this temperature for $10 \mathrm{~h}$, the mixture was then filtered through a pad of Celite and concentrated under reduced pressure. The residue was purified through column chromatography on silica gel (petroleum ether/ethyl acetate) to afford the corresponding products 3 or $\mathbf{5}$. ${ }^{\text {a }} 3$ Equiv of the alkyl bromide was used in the case of $\mathbf{3 k b}$.

\section{Analytical Data for the Products 3 and 5}

The products $\mathbf{3 a b},{ }^{10} \mathbf{3 a c},{ }^{11} \mathbf{3 a f},{ }^{12} \mathbf{3} \mathbf{a} \mathbf{i}^{13}$ and $\mathbf{3} \mathbf{a} \mathbf{t}^{14}$ are known compounds and their NMR-data are consistent with these reported in the literature.

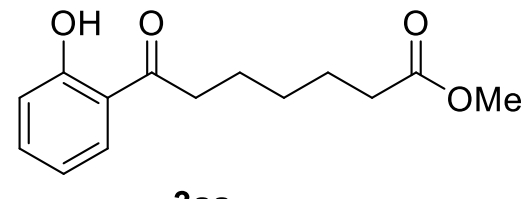

3aa

Methyl 7-(2-hydroxyphenyl)-7-oxoheptanoate (3aa) was isolated through column chromatography on silica gel with petroleum ether to the mixture eluent of petroleum ether and ethyl acetate (10:1) as a colorless oil (46 mg, $92 \%$ yield). ${ }^{1} \mathrm{H}$ NMR (400 MHz, $\left.\mathrm{CDCl}_{3}\right) \delta=12.36(\mathrm{~s}, 1 \mathrm{H}), 7.75(\mathrm{dd}, J=8.1,1.7 \mathrm{~Hz}, 1 \mathrm{H}), 7.51-7.42(\mathrm{~m}, 1 \mathrm{H}), 7.01-6.95$ (m, 1H), 6.93-6.85 (m, 1H), $3.67(\mathrm{~s}, 3 \mathrm{H}), 3.00(\mathrm{t}, J=7.4 \mathrm{~Hz}, 2 \mathrm{H}), 2.34(\mathrm{t}, J=7.4 \mathrm{~Hz}, 2 \mathrm{H}), 1.82-1.62(\mathrm{~m}, 4 \mathrm{H}), 1.48-1.36(\mathrm{~m}$, 2H) ppm; ${ }^{13} \mathrm{C}$ NMR $\left(101 \mathrm{MHz}, \mathrm{CDCl}_{3}\right) \delta=206.5,174.1,162.5,136.3,129.9,119.3,118.9,118.5,51.6,38.0,33.8,28.7,24.7$, 24.0 ppm; HRMS (ESI): calcd. for $\mathrm{C}_{14} \mathrm{H}_{19} \mathrm{O}_{4}[\mathrm{M}+\mathrm{H}]^{+}: 251.1278$ found: 251.1261 .

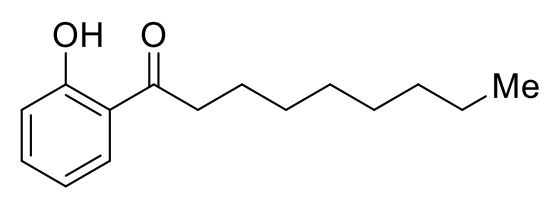

$3 a b$

1-(2-Hydroxyphenyl)nonan-1-one (3ab) was isolated through column chromatography on silica gel with petroleum ether to the mixture eluent of petroleum ether and ethyl acetate $(10: 1)$ as a colorless oil $\left(40 \mathrm{mg}, 82 \%\right.$ yield). ${ }^{1} \mathrm{H} \mathrm{NMR}\left(500 \mathrm{MHz}, \mathrm{CDCl}_{3}\right) \delta=$ $12.41(\mathrm{~s}, 1 \mathrm{H}), 7.76(\mathrm{dd}, J=8.0,1.6 \mathrm{~Hz}, 1 \mathrm{H}), 7.49-7.42(\mathrm{~m}, 1 \mathrm{H}), 6.98$ (dd, $J=8.4,1.1$ $\mathrm{Hz}, 1 \mathrm{H}), 6.93-6.87(\mathrm{~m}, 1 \mathrm{H}), 2.98(\mathrm{t}, J=7.5 \mathrm{~Hz}, 2 \mathrm{H}), 1.79-1.68(\mathrm{~m}, 2 \mathrm{H}), 1.44-1.20$ (m, $10 \mathrm{H}), 0.88(\mathrm{t}, J=6.8 \mathrm{~Hz}, 3 \mathrm{H}) . \mathrm{ppm} ;{ }^{13} \mathrm{C} \mathrm{NMR}\left(126 \mathrm{MHz}, \mathrm{CDCl}_{3}\right) \delta=207.0,162.5,136.2,130.0,119.4,118.8,118.5,38.4$, $31.9,29.42,29.35,29.2,24.6,22.7,14.1 \mathrm{ppm}$;

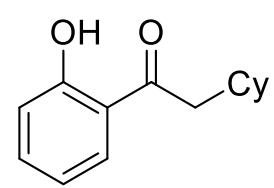

3ac

2-Cyclohexyl-1-(2-hydroxyphenyl)ethan-1-one (3ac) was isolated through column chromatography on silica gel with petroleum ether to the mixture eluent of petroleum ether and ethyl acetate (20:1) as a colorless oil (40 mg, $92 \%$ yield). ${ }^{1} \mathrm{H}$ NMR $\left(500 \mathrm{MHz}, \mathrm{CDCl}_{3}\right) \delta=12.50(\mathrm{~s}, 1 \mathrm{H}), 7.75$ (dd, $J=7.9,1.7$ $\mathrm{Hz}, 1 \mathrm{H}), 7.50-7.43(\mathrm{~m}, 1 \mathrm{H}), 6.98(\mathrm{~d}, J=8.4 \mathrm{~Hz}, 1 \mathrm{H}), 6.89(\mathrm{t}, J=7.6 \mathrm{~Hz}, 1 \mathrm{H}), 2.83(\mathrm{~d}, J=6.8 \mathrm{~Hz}, 2 \mathrm{H})$, 2.01-1.93 (m, 1H), 1.83-1.62 (m, 5H), 1.33-1.24 (m, 2H), 1.22-1.13 (m, $1 \mathrm{H}), 1.04(\mathrm{dd}, J=12.1,3.3 \mathrm{~Hz}$, 2H) ppm; ${ }^{13} \mathrm{C}$ NMR $\left(126 \mathrm{MHz}, \mathrm{CDCl}_{3}\right) \delta=206.8,162.7,136.3,130.3,119.7,118.8,118.6,45.9,35.0,33.4$ (2C), 26.2, 26.1 (2C) $\mathrm{ppm}$.

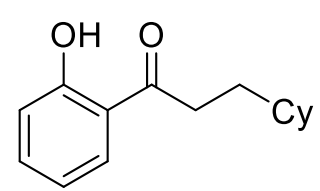

3ad

3-Cyclohexyl-1-(2-hydroxyphenyl)propan-1-one (3ad) was isolated through column chromatography on silica gel with petroleum ether to the mixture eluent of petroleum ether and ethyl acetate (20:1) as a yellow oil (42 mg, $90 \%$ yield). ${ }^{1} \mathrm{H}$ NMR $\left(500 \mathrm{MHz}, \mathrm{CDCl}_{3}\right) \delta=12.41(\mathrm{~s}, 1 \mathrm{H}), 7.76(\mathrm{dd}, J=8.1,1.6$ $\mathrm{Hz}, 1 \mathrm{H}), 7.47-7.42(\mathrm{~m}, 1 \mathrm{H}), 6.98(\mathrm{dd}, J=8.5,1.1 \mathrm{~Hz}, 1 \mathrm{H}), 6.92-6.86(\mathrm{~m}, 1 \mathrm{H}), 3.05-2.96(\mathrm{~m}, 2 \mathrm{H})$, $1.79-1.71(\mathrm{~m}, 4 \mathrm{H}), 1.69-1.60(\mathrm{~m}, 3 \mathrm{H}), 1.39-1.12(\mathrm{~m}, 4 \mathrm{H}), 1.01-0.89(\mathrm{~m}, 2 \mathrm{H}) \mathrm{ppm} ;{ }^{13} \mathrm{C}$ NMR $(126$ $\left.\mathrm{MHz}, \mathrm{CDCl}_{3}\right) \delta=207.4,162.5,136.2,130.0,119.3,118.9,118.6,37.4,36.0,33.2$ (2C), 32.0, 26.5, 26.3 (2C) ppm; HRMS 
<smiles>CCCCC(=O)c1ccccc1O</smiles>

3ae

3-Ethyl-1-(2-hydroxyphenyl)heptan-1-one (3ae) was isolated through column chromatography on silica gel with petroleum ether to the mixture eluent of petroleum ether and ethyl acetate (20:1) as a yellow oil (41 mg, $87 \%$ yield). ${ }^{1} \mathrm{H}$ NMR $\left(500 \mathrm{MHz}, \mathrm{CDCl}_{3}\right) \delta=12.50(\mathrm{~s}, 1 \mathrm{H}), 7.77(\mathrm{dd}, J=8.1$, $1.6 \mathrm{~Hz}, 1 \mathrm{H}), 7.50-7.40(\mathrm{~m}, 1 \mathrm{H}), 6.98(\mathrm{~d}, J=8.4 \mathrm{~Hz}, 1 \mathrm{H}), 6.89(\mathrm{t}, J=7.6 \mathrm{~Hz}, 1 \mathrm{H}), 2.89(\mathrm{~d}, J=6.8$ $\mathrm{Hz}, 2 \mathrm{H}), 2.07-2.00(\mathrm{~m}, 1 \mathrm{H}), 1.43-1.35(\mathrm{~m}, 3 \mathrm{H}), 1.34-1.24(\mathrm{~m}, 5 \mathrm{H}), 0.91(\mathrm{t}, J=7.5 \mathrm{~Hz}, 3 \mathrm{H}), 0.89$ (t, $J=6.9 \mathrm{~Hz}, 3 \mathrm{H}) \mathrm{ppm} ;{ }^{13} \mathrm{C} \mathrm{NMR}\left(126 \mathrm{MHz}, \mathrm{CDCl}_{3}\right) \delta=207.3,162.6,136.2,130.1,119.7,118.8,118.6,42.7,36.1,33.2,28.9$, 26.4, 23.0, 14.1, 10.9 ppm; HRMS (ESI): calcd. for $\mathrm{C}_{15} \mathrm{H}_{23} \mathrm{O}_{2}[\mathrm{M}+\mathrm{H}]^{+}: 235.1693$, found: 235.1693.<smiles>O=C(Cc1ccccc1)c1ccccc1O</smiles>

$119.0,118.6,40.1,30.0 \mathrm{ppm}$.

1-(2-Hydroxyphenyl)-3-phenylpropan-1-one (3af) was isolated through column chromatography on silica gel (petroleum ether: $\mathrm{EtOAc}=20: 1)$ as a colorless oil $\left(32 \mathrm{mg}, 71 \%\right.$ yield). ${ }^{1} \mathrm{H}$ NMR $\left(400 \mathrm{MHz}, \mathrm{CDCl}_{3}\right)$ $\delta=12.30(\mathrm{~s}, 1 \mathrm{H}), 7.74(\mathrm{dd}, J=8.1,1.7 \mathrm{~Hz}, 1 \mathrm{H}), 7.49-7.43(\mathrm{~m}, 1 \mathrm{H}), 7.35-7.28(\mathrm{~m}, 2 \mathrm{H}), 7.27-7.18(\mathrm{~m}, 3 \mathrm{H})$, $6.98(\mathrm{dd}, J=8.4,1.2 \mathrm{~Hz}, 1 \mathrm{H}), 6.89-6.83(\mathrm{~m}, 1 \mathrm{H}), 3.33(\mathrm{t}, J=7.3 \mathrm{~Hz}, 2 \mathrm{H}), 3.07$ (t, $J=7.4 \mathrm{~Hz}, 2 \mathrm{H}) \mathrm{ppm}$; ${ }^{13} \mathrm{C}$ NMR $\left(101 \mathrm{MHz}, \mathrm{CDCl}_{3}\right) \delta=205.4,162.5,140.7,136.4,129.8,128.6$ (2C), 128.4 (2C), 126.4, 119.3,<smiles>O=C(CCCBr)c1ccccc1O</smiles>

3ag

1-(2-Hydroxyphenyl)-5-phenylpentan-1-one (3ag) was isolated through column chromatography on silica gel (petroleum ether: EtOAc $=20: 1)$ as a colorless oil ( $40 \mathrm{mg}, 78 \%$ yield). ${ }^{1} \mathrm{H}$ NMR (400 $\left.\mathrm{MHz}, \mathrm{CDCl}_{3}\right) \delta=12.38(\mathrm{~s}, 1 \mathrm{H}), 7.72(\mathrm{~d}, J=8.0 \mathrm{~Hz}, 1 \mathrm{H}), 7.45(\mathrm{~s}, 1 \mathrm{H}), 7.28(\mathrm{t}, J=7.4 \mathrm{~Hz}, 2 \mathrm{H}), 7.20$ $-7.13(\mathrm{~m}, 3 \mathrm{H}), 6.97(\mathrm{~d}, J=8.4 \mathrm{~Hz}, 1 \mathrm{H}), 6.87(\mathrm{t}, J=7.6 \mathrm{~Hz}, 1 \mathrm{H}), 2.99$ (t, $J=7.0 \mathrm{~Hz}, 2 \mathrm{H}), 2.67(\mathrm{t}, J$ $=7.3 \mathrm{~Hz}, 2 \mathrm{H}), 1.86-1.63(\mathrm{~m}, 4 \mathrm{H}) \mathrm{ppm} ;{ }^{13} \mathrm{C} \mathrm{NMR}\left(101 \mathrm{MHz}, \mathrm{CDCl}_{3}\right) \delta=206.6,162.5,142.1,136.3$, 130.0, 128.42 (2C), 128.39 (2C), 125.9, 119.3, 118.9, 118.6, 38.2, 35.8, 31.0, 24.1 ppm; HRMS (ESI): calcd. for $\mathrm{C}_{17} \mathrm{H}_{19} \mathrm{O}_{2}$ $[\mathrm{M}+\mathrm{H}]^{+}: 255.1380$, found: 255.1378 .

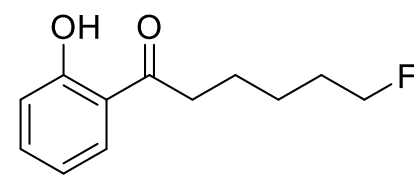

$3 a h$

6-Fluoro-1-(2-hydroxyphenyl)hexan-1-one (3ah) was isolated through column chromatography on silica gel with petroleum ether to the mixture eluent of petroleum ether and ethyl acetate (20:1) as a yellow oil (35 mg, $83 \%$ yield). ${ }^{1} \mathrm{H}$ NMR $\left(500 \mathrm{MHz}, \mathrm{CDCl}_{3}\right) \delta=12.36$ (s, 1H), $7.76(\mathrm{~d}, J=8.0 \mathrm{~Hz}, 1 \mathrm{H}), 7.47(\mathrm{t}, J=7.8 \mathrm{~Hz}, 1 \mathrm{H}), 6.98(\mathrm{~d}, J=8.4 \mathrm{~Hz}, 1 \mathrm{H}), 6.90$ (t, $J=$ $7.6 \mathrm{~Hz}, 1 \mathrm{H}), 4.47$ (dt, $J=47.3,6.0 \mathrm{~Hz}, 2 \mathrm{H}), 3.02$ (t, $J=7.3 \mathrm{~Hz}, 2 \mathrm{H}), 1.83-1.76(\mathrm{~m}, 3 \mathrm{H}), 1.76-$ $1.71(\mathrm{~m}, 1 \mathrm{H}), 1.57-1.47(\mathrm{~m}, 2 \mathrm{H}) \mathrm{ppm} ;{ }^{13} \mathrm{C}$ NMR $\left(126 \mathrm{MHz}, \mathrm{CDCl}_{3}\right) \delta=206.5,162.5,136.3,129.9,119.3,118.9,118.6,84.5$ $\left(\mathrm{C}-\mathrm{F},{ }^{1} J_{\mathrm{C}-\mathrm{F}}=164.4 \mathrm{~Hz}\right), 83.2\left(\mathrm{C}-\mathrm{F},{ }^{1} J_{\mathrm{C}-\mathrm{F}}=164.4 \mathrm{~Hz}\right), 38.1,30.4\left(\mathrm{C}-\mathrm{F},{ }^{2} J_{\mathrm{C}-\mathrm{F}}=19.4 \mathrm{~Hz}\right), 30.2\left(\mathrm{C}-\mathrm{F},{ }^{2} J_{\mathrm{C}-\mathrm{F}}=19.4 \mathrm{~Hz}\right), 25.03$ $\left(\mathrm{C}-\mathrm{F},{ }^{3} J_{\mathrm{C}-\mathrm{F}}=5.4 \mathrm{~Hz}\right), 24.99\left(\mathrm{C}-\mathrm{F},{ }^{3} J_{\mathrm{C}-\mathrm{F}}=5.4 \mathrm{~Hz}\right), 24.0 \mathrm{ppm} ;{ }^{19} \mathrm{~F}$ NMR $\left(376 \mathrm{MHz}, \mathrm{CDCl}_{3}\right) \delta=-218.25--218.73(\mathrm{~m}, 1 \mathrm{~F})$; HRMS (ESI): calcd. for $\mathrm{C}_{12} \mathrm{H}_{16} \mathrm{FO}_{2}[\mathrm{M}+\mathrm{H}]^{+}: 211.1129$, found:211.1125.

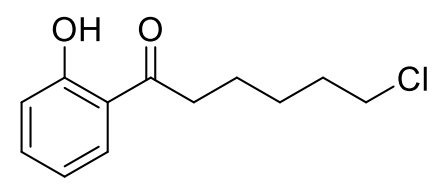

3ai

6-Chloro-1-(2-hydroxyphenyl)hexan-1-one (3ai) was isolated through column chromatography on silica gel with petroleum ether to the mixture eluent of petroleum ether and ethyl acetate (20:1) as a colorless oil (35 mg, $77 \%$ yield). ${ }^{1} \mathrm{H}$ NMR $\left(400 \mathrm{MHz}, \mathrm{CDCl}_{3}\right)$ $\delta=12.34(\mathrm{~s}, 1 \mathrm{H}), 7.76(\mathrm{dd}, J=8.0,1.7 \mathrm{~Hz}, 1 \mathrm{H}), 7.55-7.41(\mathrm{~m}, 1 \mathrm{H}), 6.98(\mathrm{~d}, J=8.4 \mathrm{~Hz}, 1 \mathrm{H})$, $6.90(\mathrm{t}, J=7.6 \mathrm{~Hz}, 1 \mathrm{H}), 3.56(\mathrm{t}, J=6.6 \mathrm{~Hz}, 2 \mathrm{H}), 3.02(\mathrm{t}, J=7.3 \mathrm{~Hz}, 2 \mathrm{H}), 1.88-1.70(\mathrm{~m}, 4 \mathrm{H})$, 1.61-1.51 (m, 2H) ppm; ${ }^{13} \mathrm{C}$ NMR $\left(101 \mathrm{MHz}, \mathrm{CDCl}_{3}\right) \delta=206.3,162.5,136.3,129.9,119.3,118.9,118.6,44.8,38.0,32.4$, 26.6, $23.6 \mathrm{ppm}$; 


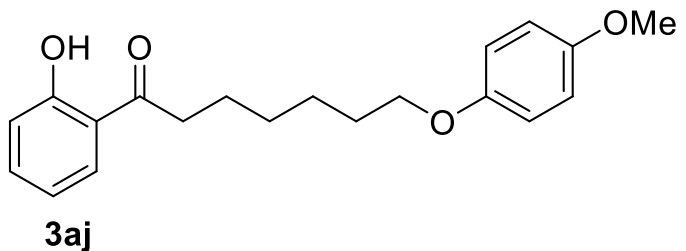

3aj
1-(2-Hydroxyphenyl)-7-(4-methoxyphenoxy)heptan-1-one

(3aj) was isolated through column chromatography on silica gel with petroleum ether to the mixture eluent of petroleum ether and ethyl acetate $(5: 1)(53 \mathrm{mg}, 81 \%$ yield). ${ }^{1} \mathrm{H}$ NMR $\left(400 \mathrm{MHz}, \mathrm{CDCl}_{3}\right) \delta=12.39(\mathrm{~s}, 1 \mathrm{H}), 7.76(\mathrm{dd}, J=8.0,1.7$ $\mathrm{Hz}, 1 \mathrm{H}), 7.46$ (t, $J=1.5 \mathrm{~Hz}, 1 \mathrm{H}), 6.98$ (dd, $J=8.4,1.2 \mathrm{~Hz}, 1 \mathrm{H}), 6.90(\mathrm{~d}, J$ $=8.2 \mathrm{~Hz}, 1 \mathrm{H}), 6.82(\mathrm{~s}, 4 \mathrm{H}), 3.91(\mathrm{t}, J=6.4 \mathrm{~Hz}, 2 \mathrm{H}), 3.76(\mathrm{~s}, 3 \mathrm{H}), 3.00(\mathrm{t}, J$

$=7.4 \mathrm{~Hz}, 2 \mathrm{H}), 1.83-1.72(\mathrm{~m}, 4 \mathrm{H}), 1.57-1.42(\mathrm{~m}, 4 \mathrm{H}) \mathrm{ppm} ;{ }^{13} \mathrm{C}$ NMR $\left(101 \mathrm{MHz}, \mathrm{CDCl}_{3}\right) \delta=206.8,162.5,153.7,153.2,136.3$, 130.0, 119.3, 118.9, 118.6, 115.4 (2C), 114.6 (2C), 68.4, 55.8, 38.2, 29.2, 29.1, 26.0, 24.4 ppm; HRMS (ESI): calcd. for $\mathrm{C}_{20} \mathrm{H}_{25} \mathrm{O}_{4}[\mathrm{M}+\mathrm{H}]^{+}: 329.1747$, found: 329.1750 .

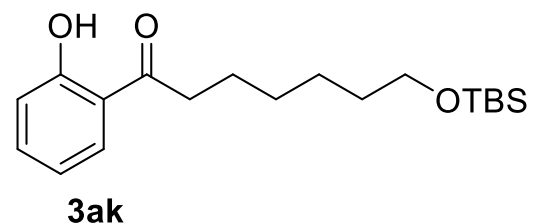

$3 a k$

7-((tert-Butyldimethylsilyl)oxy)-1-(2-hydroxyphenyl)heptan-1-one (3ak) was isolated through column chromatography on silica gel with petroleum ether to the mixture eluent of petroleum ether and ethyl acetate (10:1) as a colorless oil (66 mg, $98 \%$ yield). ${ }^{1} \mathrm{H}$ NMR $\left(400 \mathrm{MHz}, \mathrm{CDCl}_{3}\right) \delta=12.41(\mathrm{~s}, 1 \mathrm{H}), 7.76(\mathrm{dd}, J=8.0,1.7 \mathrm{~Hz}, 1 \mathrm{H}), 7.49-7.42$ $(\mathrm{m}, 1 \mathrm{H}), 7.00-6.96(\mathrm{~m}, 1 \mathrm{H}), 6.93-6.86(\mathrm{~m}, 1 \mathrm{H}), 3.61(\mathrm{t}, J=6.4 \mathrm{~Hz}, 2 \mathrm{H}), 2.99(\mathrm{t}, J=7.4$ $\mathrm{Hz}, 2 \mathrm{H}), 1.84-1.66(\mathrm{~m}, 2 \mathrm{H}), 1.60-1.48(\mathrm{~m}, 2 \mathrm{H}), 1.47-1.34(\mathrm{~m}, 4 \mathrm{H}), 0.89(\mathrm{~s}, 9 \mathrm{H}), 0.05(\mathrm{~s}, 6 \mathrm{H}) ;{ }^{13} \mathrm{C}$ NMR $\left(101 \mathrm{MHz}, \mathrm{CDCl}_{3}\right)$ $\delta=206.9,162.5,136.2,130.0,119.3,118.8,118.5,63.1,38.3,32.7,29.1,26.0(3 \mathrm{C}), 25.7,24.5,18.4,-5.6(2 \mathrm{C}) \mathrm{ppm}$; HRMS (ESI): calcd. for $\mathrm{C}_{19} \mathrm{H}_{33} \mathrm{O}_{3} \mathrm{Si}[\mathrm{M}+\mathrm{H}]^{+}: 337.2193$, found: 337.2189 .

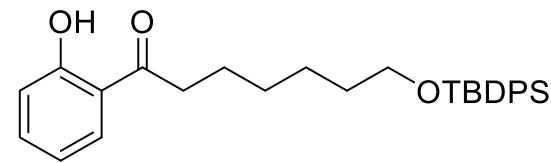

3al

7-((tert-Butyldiphenylsilyl)oxy)-1-(2-hydroxyphenyl)heptan-1-one (3al) was isolated through column chromatography on silica gel with petroleum ether to the mixture eluent of petroleum ether and ethyl acetate (10:1) as a colorless oil ( $83 \mathrm{mg}, 90 \%$ yield). ${ }^{1} \mathrm{H}$ NMR $\left(400 \mathrm{MHz}, \mathrm{CDCl}_{3}\right) \delta=12.42(\mathrm{~s}, 1 \mathrm{H}), 7.74(\mathrm{dd}, J=8.1,1.7 \mathrm{~Hz}, 1 \mathrm{H}), 7.67(\mathrm{dd}$, $J=7.4,1.9 \mathrm{~Hz}, 4 \mathrm{H}), 7.45-7.32(\mathrm{~m}, 7 \mathrm{H}), 7.00-6.96(\mathrm{~m}, 1 \mathrm{H}), 6.90-6.84(\mathrm{~m}, 1 \mathrm{H}), 3.67$ $(\mathrm{t}, J=6.3 \mathrm{~Hz}, 2 \mathrm{H}), 2.95(\mathrm{t}, J=7.5 \mathrm{~Hz}, 2 \mathrm{H}), 1.75-1.68(\mathrm{~m}, 2 \mathrm{H}), 1.63-1.51(\mathrm{~m}, 2 \mathrm{H}), 1.47-1.32(\mathrm{~m}, 4 \mathrm{H}), 1.05(\mathrm{~s}, 9 \mathrm{H}) \mathrm{ppm} ;{ }^{13} \mathrm{C}$ NMR $\left(101 \mathrm{MHz}, \mathrm{CDCl}_{3}\right) \delta=206.9,162.6,136.2,135.6$ (4C), 134.2, 134.1, 130.0, 129.6, 129.5, 127.6 (4C), 119.4, 118.9, 118.6, 63.8, 38.3, 2.4, 29.1, 26.9 (3C), 25.7, 24.5, 19.3 ppm; HRMS (ESI): calcd. for $\mathrm{C}_{29} \mathrm{H}_{37} \mathrm{O}_{3} \mathrm{Si}[\mathrm{M}+\mathrm{H}]^{+}: 461.2506$, found: 461.2536 .

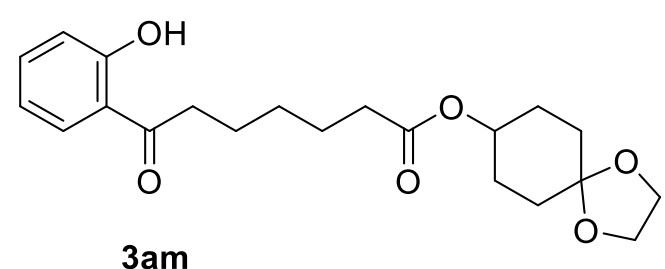

3am
1,4-Dioxaspiro[4.5]decan-8-yl 7-(2-hydroxyphenyl)-7-oxoheptanoate (3am) was isolated through column chromatography on silica gel with petroleum ether to the mixture eluent of petroleum ether and ethyl acetate (5:1) as a white solid (53 mg, $71 \%$ yield). ${ }^{1} \mathrm{H}$ NMR (400 MHz, $\left.\mathrm{CDCl}_{3}\right) \delta=12.36(\mathrm{~s}, 1 \mathrm{H}), 7.76$ (dd, $J=8.1,1.7 \mathrm{~Hz}, 1 \mathrm{H}), 7.49-7.42(\mathrm{~m}, 1 \mathrm{H}), 6.98(\mathrm{dd}, J=8.4,1.2 \mathrm{~Hz}, 1 \mathrm{H})$, 6.93-6.86 (m, 1H), 4.94-4.81 (m, 1H), 3.97-3.87 (m, 4H), $3.00(\mathrm{t}, J=7.4 \mathrm{~Hz}$,

$2 \mathrm{H}), 2.33(\mathrm{t}, J=7.4 \mathrm{~Hz}, 2 \mathrm{H}), 1.92-1.70(\mathrm{~m}, 8 \mathrm{H}), 1.72-1.55(\mathrm{~m}, 4 \mathrm{H}), 1.48-1.38(\mathrm{~m}, 2 \mathrm{H}) \mathrm{ppm} ;{ }^{13} \mathrm{C} \mathrm{NMR}(101 \mathrm{MHz}, \mathrm{CDCl} 3) \delta=$ 206.5, 173.1 162.5, 136.3, 129.9, 118.9, 118.5, 108.0, 70.0, 64.4 (2C), 64.3 (2C), 38.0, 34.5, 31.3, 28.7, 28.4 (2C), 24.8, 24.0 ppm; HRMS (ESI): calcd. for $\mathrm{C}_{21} \mathrm{H}_{29} \mathrm{O}_{6}[\mathrm{M}+\mathrm{H}]^{+}: 377.1959$, found: 377.1963 .

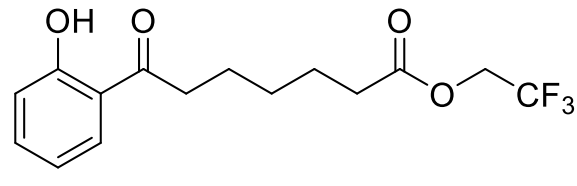

3an

2,2,2-Trifluoroethyl 7-(2-hydroxyphenyl)-7-oxoheptanoate (3an) was isolated through column chromatography on silica gel with petroleum ether to the mixture eluent of petroleum ether and ethyl acetate (20:1) as a colorless oil (49 $\mathrm{mg}, 77 \%$ yield). ${ }^{1} \mathrm{H}$ NMR $\left(500 \mathrm{MHz}, \mathrm{CDCl}_{3}\right) \delta=12.35(\mathrm{~s}, 1 \mathrm{H}), 7.75(\mathrm{dd}, J=8.1,1.6 \mathrm{~Hz}$, $1 \mathrm{H}), 7.50-7.44(\mathrm{~m}, 1 \mathrm{H}), 6.98(\mathrm{dd}, J=8.4,1.1 \mathrm{~Hz}, 1 \mathrm{H}), 6.93-6.86(\mathrm{~m}, 1 \mathrm{H}), 4.47(\mathrm{q}$, $J=8.5 \mathrm{~Hz}, 2 \mathrm{H}), 3.01(\mathrm{t}, J=7.4 \mathrm{~Hz}, 2 \mathrm{H}), 2.46(\mathrm{t}, J=7.5 \mathrm{~Hz}, 2 \mathrm{H}), 1.83-1.69(\mathrm{~m}, 4 \mathrm{H}), 1.48-1.39(\mathrm{~m}, 2 \mathrm{H}) \mathrm{ppm} ;{ }^{13} \mathrm{C}$ NMR $(126$ $\left.\mathrm{MHz}, \mathrm{CDCl}_{3}\right) \delta=206.4,171.9,162.5,136.3,129.9,123.0$ (q, $\left.J=277.4 \mathrm{~Hz}\right), 119.3,118.9,118.6,60.2(\mathrm{q}, J=36.6 \mathrm{~Hz}), 37.9$, 
33.4, 28.5, 24.5, $23.8 \mathrm{ppm} ;{ }^{19} \mathrm{~F}$ NMR (376 MHz, $\left.\mathrm{CDCl}_{3}\right) \delta=-73.78--73.92$ (m, 3F)ppm; HRMS (ESI): calcd. for $\mathrm{C}_{15} \mathrm{H}_{17} \mathrm{O}_{4} \mathrm{~F}_{3} \mathrm{Na}[\mathrm{M}+\mathrm{Na}]^{+}: 341.0971$, found:341.0979.

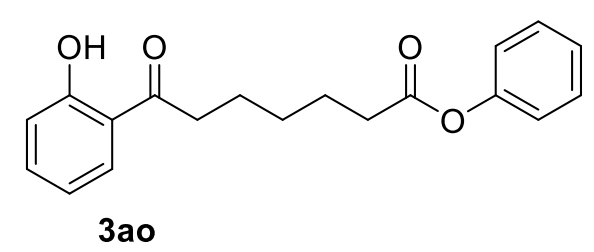

Phenyl 7-(2-hydroxyphenyl)-7-oxoheptanoate (3ao) was isolated through column chromatography on silica gel with petroleum ether to the mixture eluent of petroleum ether and ethyl acetate (20:1) (49 mg, $78 \%$ yield). ${ }^{1} \mathrm{H}$ NMR (500 MHz, $\left.\mathrm{CDCl}_{3}\right) \delta=12.37(\mathrm{~s}, 1 \mathrm{H}), 7.76(\mathrm{dd}, J=8.0,1.6 \mathrm{~Hz}, 1 \mathrm{H}), 7.49-7.44(\mathrm{~m}, 1 \mathrm{H}), 7.40-$ $7.35(\mathrm{~m}, 2 \mathrm{H}), 7.24-7.18(\mathrm{~m}, 1 \mathrm{H}), 7.11-7.05(\mathrm{~m}, 2 \mathrm{H}), 6.99(\mathrm{dd}, J=8.4,1.1 \mathrm{~Hz}, 1 \mathrm{H})$, 6.92-6.87 (m, 1H), 3.03 (t, $J=7.3 \mathrm{~Hz}, 2 \mathrm{H}), 2.60(\mathrm{t}, J=7.4 \mathrm{~Hz}, 2 \mathrm{H}), 1.86-1.76(\mathrm{~m}$, 4H), 1.60-1.48 (m, 2H) ppm; ${ }^{13} \mathrm{C}$ NMR $\left(126 \mathrm{MHz}, \mathrm{CDCl}_{3}\right) \delta=206.5,172.1,162.5,150.7,136.3,130.0,129.5(2 \mathrm{C}), 125.8$, 121.6 (2C), 119.3, 118.9, 118.6, 38.0, 34.2, 29.0, 24.7, 23.9 ppm; HRMS (ESI): calcd. for $\mathrm{C}_{19} \mathrm{H}_{21} \mathrm{O}_{4}[\mathrm{M}+\mathrm{H}]^{+}: 313.1434$, found: 313.1438 .

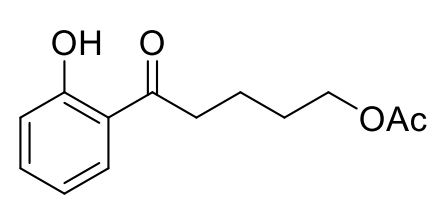

$3 a p$

5-(2-Hydroxyphenyl)-5-oxopentyl acetate (3ap) was isolated through column chromatography on silica gel with petroleum ether to the mixture eluent of petroleum ether and ethyl acetate (10:1) as a colorless oil (34 mg, $73 \%$ yield). ${ }^{1} \mathrm{H}$ NMR (400 MHz, $\left.\mathrm{CDCl}_{3}\right) \delta=12.32(\mathrm{~s}, 1 \mathrm{H})$, $7.76(\mathrm{dd}, J=8.0,1.7 \mathrm{~Hz}, 1 \mathrm{H}), 7.52-7.44(\mathrm{~m}, 1 \mathrm{H}), 6.98(\mathrm{dd}, J=8.5,1.2 \mathrm{~Hz}, 1 \mathrm{H}), 6.94-6.86(\mathrm{~m}$, $1 \mathrm{H}), 4.12(\mathrm{t}, J=6.3 \mathrm{~Hz}, 2 \mathrm{H}), 3.04$ (t, $J=7.1 \mathrm{~Hz}, 2 \mathrm{H}), 2.05$ (s, 3H), 1.91-1.71 (m, 4H) ppm;

${ }^{13} \mathrm{C}$ NMR $\left(101 \mathrm{MHz}, \mathrm{CDCl}_{3}\right) \delta=206.1,171.2,162.5,136.4,129.8,119.3,118.9,118.6,64.0$, 37.6, 28.1, 21.0, 20.7 ppm; HRMS (ESI): calcd. for $\mathrm{C}_{13} \mathrm{H}_{16} \mathrm{O}_{4}[\mathrm{M}+\mathrm{Na}]^{+}: 259.0941$, found:259.0947.

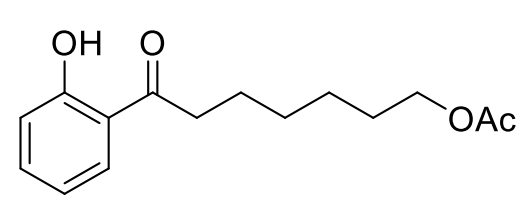

$3 a q$

7-(2-Hydroxyphenyl)-7-oxoheptyl acetate (3aq) was isolated through column chromatography on silica gel with petroleum ether to the mixture eluent of petroleum ether and ethyl acetate (10:1) as a colorless oil (47 mg, $89 \%$ yield). ${ }^{1} \mathrm{H}$ NMR (400 MHz, $\left.\mathrm{CDCl}_{3}\right) \delta=12.38(\mathrm{~s}, 1 \mathrm{H}), 7.76(\mathrm{dd}, J=8.1,1.7 \mathrm{~Hz}, 1 \mathrm{H}), 7.50-7.42(\mathrm{~m}, 1 \mathrm{H}), 6.98(\mathrm{dd}, J$ $=8.4,1.1 \mathrm{~Hz}, 1 \mathrm{H}), 6.94-6.86(\mathrm{~m}, 1 \mathrm{H}), 4.07(\mathrm{t}, J=6.7 \mathrm{~Hz}, 2 \mathrm{H}), 3.00(\mathrm{t}, J=7.4 \mathrm{~Hz}, 2 \mathrm{H})$, $2.05(\mathrm{~s}, 3 \mathrm{H}), 1.85-1.58(\mathrm{~m}, 4 \mathrm{H}), 1.51-1.37(\mathrm{~m}, 4 \mathrm{H}) \mathrm{ppm} ;{ }^{13} \mathrm{C} \mathrm{NMR}\left(101 \mathrm{MHz}, \mathrm{CDCl}_{3}\right) \delta=206.7,171.2,162.5,136.3,129.9$, 119.3, 118.9, 118.6, 64.4, 38.2, 28.9, 28.5, 25.8, 24.3, 21.0 ppm; HRMS (ESI): calcd. for $\mathrm{C}_{15} \mathrm{H}_{21} \mathrm{O}_{4}[\mathrm{M}+\mathrm{H}]^{+}: 265.1434$, found:265.1439.

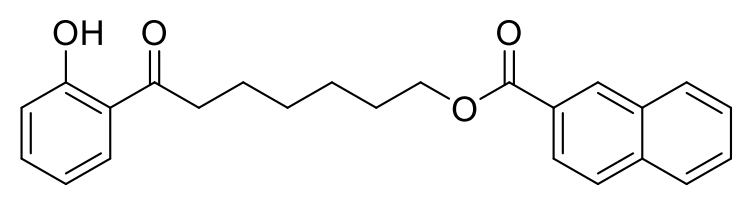

3ar

7-(2-Hydroxyphenyl)-7-oxoheptyl 2-naphthoate (3ar) was isolated through column chromatography on silica gel with petroleum ether to the mixture eluent of petroleum ether and ethyl acetate (10:1) as a colorless oil (48 mg, $64 \%$ yield). ${ }^{1} \mathrm{H}$ NMR (400 MHz, $\left.\mathrm{CDCl}_{3}\right) \delta=12.39(\mathrm{~s}, 1 \mathrm{H})$, $8.60(\mathrm{~d}, J=1.6 \mathrm{~Hz}, 1 \mathrm{H}), 8.06(\mathrm{dd}, J=8.6,1.7 \mathrm{~Hz}, 1 \mathrm{H}), 7.95(\mathrm{~d}, J=8.0$ $\mathrm{Hz}, 1 \mathrm{H}), 7.90-7.85(\mathrm{~m}, 2 \mathrm{H}), 7.74(\mathrm{dd}, J=8.1,1.7 \mathrm{~Hz}, 1 \mathrm{H}), 7.61-7.50(\mathrm{~m}, 2 \mathrm{H}), 7.49-7.41(\mathrm{~m}, 1 \mathrm{H}), 6.98(\mathrm{dd}, J=8.4,1.1 \mathrm{~Hz}$, $1 \mathrm{H}), 6.90-6.81(\mathrm{~m}, 1 \mathrm{H}), 4.39(\mathrm{t}, J=6.6 \mathrm{~Hz}, 2 \mathrm{H}), 3.00(\mathrm{t}, J=7.4 \mathrm{~Hz}, 2 \mathrm{H}), 1.91-1.73(\mathrm{~m}, 4 \mathrm{H}), 1.61-1.42(\mathrm{~m}, 4 \mathrm{H}) \mathrm{ppm} ;{ }^{13} \mathrm{C}$ NMR $\left(101 \mathrm{MHz}, \mathrm{CDCl}_{3}\right) \delta=206.7,166.8,162.5,136.3,135.5,132.5,131.0,130.0,129.4,128.22,128.15,127.8,127.7$, 126.6, 125.3, 119.3, 118.9, 118.6, 65.1, 38.2, 29.0, 28.7, 26.0, 24.3 ppm; HRMS (EI): calcd. for $\mathrm{C}_{24} \mathrm{H}_{25} \mathrm{O}_{4}[\mathrm{M}+\mathrm{H}]^{+}: 377.1747$, found: 377.1753 .

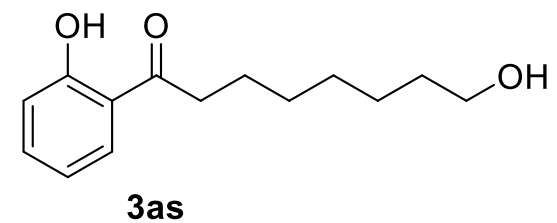

8-Hydroxy-1-(2-hydroxyphenyl)octan-1-one (3as) was isolated through column chromatography on silica gel with petroleum ether to the mixture eluent of petroleum ether and ethyl acetate (10:1) as a colorless oil (24 mg, $51 \%$ yield). ${ }^{1} \mathrm{H}$ NMR (500 $\left.\mathrm{MHz}, \mathrm{CDCl}_{3}\right) \delta=12.39(\mathrm{~s}, 1 \mathrm{H}), 7.77(\mathrm{dd}, J=8.1,1.7 \mathrm{~Hz}, 1 \mathrm{H}), 7.50-7.43(\mathrm{~m}, 1 \mathrm{H}), 6.98$ (dd, $J=8.4,1.1 \mathrm{~Hz}, 1 \mathrm{H}), 6.94-6.87(\mathrm{~m}, 1 \mathrm{H}), 3.65(\mathrm{t}, J=6.6 \mathrm{~Hz}, 2 \mathrm{H}), 2.99$ (t, $J=7.4$ 
$\mathrm{Hz}, 2 \mathrm{H}), 1.81-1.70(\mathrm{~m}, 2 \mathrm{H}), 1.57(\mathrm{q}, J=6.8 \mathrm{~Hz}, 2 \mathrm{H}), 1.44-1.34(\mathrm{~m}, 6 \mathrm{H}) \mathrm{ppm} ;{ }^{13} \mathrm{C}$ NMR $\left(126 \mathrm{MHz}, \mathrm{CDCl}_{3}\right) \delta=206.9,162.5$, 136.2, 130.0, 119.3, 118.9, 118.6, 63.0, 38.3, 32.7, 29.24, 29.21, 25.6, 24.4 ppm; HRMS (ESI): calcd. for $\mathrm{C}_{14} \mathrm{H}_{21} \mathrm{O}_{3}[\mathrm{M}+\mathrm{H}]^{+}$: 237.1485, found: 237.1487 .<smiles>O=C(Cl)c1ccccc1O</smiles>

3at

Cyclohexyl(2-hydroxyphenyl)methanone (3at) was isolated through column chromatography on silica gel (petroleum ether: $\mathrm{EtOAc}=20: 1)$ as a colorless oil $\left(26 \mathrm{mg}, 64 \%\right.$ yield). ${ }^{1} \mathrm{H}$ NMR $\left(400 \mathrm{MHz}, \mathrm{CDCl}_{3}\right) \delta=12.59$ (s, 1H), 7.81-7.74 (m, 1H), 7.49-7.42 (m, 1H), $6.99(\mathrm{~d}, J=8.4 \mathrm{~Hz}, 1 \mathrm{H}), 6.90(\mathrm{t}, J=7.6 \mathrm{~Hz}, 1 \mathrm{H}), 3.38-3.23$ (m, 1H), 1.93-1.83 (m, 4H), 1.80-1.71 (m, 1H), $1.55(\mathrm{dd}, J=12.1,3.4 \mathrm{~Hz}, 2 \mathrm{H}), 1.47-1.36(\mathrm{~m}, 2 \mathrm{H}), 1.34-1.20$ $(\mathrm{m}, 1 \mathrm{H}) \mathrm{ppm} ;{ }^{13} \mathrm{C} \mathrm{NMR}\left(101 \mathrm{MHz}, \mathrm{CDCl}_{3}\right) \delta=210.2,163.2,136.2,129.8,118.78,118.76,118.2,45.3,29.6$ (2C), $25.9,25.8(2 \mathrm{C}) \mathrm{ppm}$;

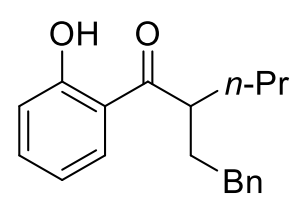

3au

1-(2-Hydroxyphenyl)-2-phenethylpentan-1-one (3au) was isolated through column chromatography on silica gel (petroleum ether: EtOAc $=20: 1)$ as a colorless oil $(28 \mathrm{mg}, 50 \%$ yield $) .{ }^{1} \mathrm{H}$ NMR $(500 \mathrm{MHz}$, $\left.\mathrm{CDCl}_{3}\right) \delta=12.69(\mathrm{~s}, 1 \mathrm{H}), 7.60(\mathrm{dd}, J=8.1,1.5 \mathrm{~Hz}, 1 \mathrm{H}), 7.51-7.44(\mathrm{~m}, 1 \mathrm{H}), 7.29-7.23(\mathrm{~m}, 2 \mathrm{H}), 7.22-$ $7.16(\mathrm{~m}, 1 \mathrm{H}), 7.15-7.09(\mathrm{~m}, 2 \mathrm{H}), 7.00(\mathrm{dd}, J=8.4,1.1 \mathrm{~Hz}, 1 \mathrm{H}), 6.90-6.81(\mathrm{~m}, 1 \mathrm{H}), 3.53-3.36(\mathrm{~m}, 1 \mathrm{H})$, 2.67-2.52 (m, 2H), 2.19-2.04 (m, 1H), 1.86-1.73 (m, 2H), 1.60-1.47 (m, 1H), 1.29 (q, $J=7.6 \mathrm{~Hz}, 2 \mathrm{H})$, $0.87(\mathrm{t}, J=7.3 \mathrm{~Hz}, 3 \mathrm{H}) \mathrm{ppm} ;{ }^{13} \mathrm{C} \mathrm{NMR}\left(101 \mathrm{MHz}, \mathrm{CDCl}_{3}\right) \delta=210.6,163.1,141.5,136.4,129.9,128.5(2 \mathrm{C}), 128.4$ (2C), 126.0, 119.6, 118.8, 118.7, 44.4, 34.9, 34.0, 33.7, 20.7, 14.2 ppm; HRMS (ESI): calcd. for $\mathrm{C}_{19} \mathrm{H}_{23} \mathrm{O}_{2}[\mathrm{M}+\mathrm{H}]^{+}: 283.1693$ found: 283.1697.<smiles>CCCC1CCC(C)CC1C(=O)c1ccccc1O</smiles>

$3 a v$

(2-Hydroxyphenyl)((1R,2S,5R)-2-isopropyl-5-methylcyclohexyl)methanone (3av) was isolated through column chromatography on silica gel (petroleum ether: $\mathrm{EtOAc}=5: 1)$ as a colorless oil $(37 \mathrm{mg}$, $71 \%$ yield). ${ }^{1} \mathrm{H}$ NMR $\left(500 \mathrm{MHz}, \mathrm{CDCl}_{3}\right) \delta=12.78(\mathrm{~s}, 1 \mathrm{H}), 7.85(\mathrm{dd}, J=8.1,1.5 \mathrm{~Hz}, 1 \mathrm{H}), 7.50-7.45$ (m, 1H), $7.00(\mathrm{dd}, J=8.4,1.1 \mathrm{~Hz}, 1 \mathrm{H}), 6.94-6.87(\mathrm{~m}, 1 \mathrm{H}), 3.40(\mathrm{~d}, J=3.3 \mathrm{~Hz}, 1 \mathrm{H}), 1.90-1.82(\mathrm{~m}, 2 \mathrm{H})$, $1.83-1.75(\mathrm{~m}, 2 \mathrm{H}), 1.61(\mathrm{~s}, 1 \mathrm{H}), 1.55-1.42(\mathrm{~m}, 1 \mathrm{H}), 1.20-1.10(\mathrm{~m}, 2 \mathrm{H}), 1.08-0.97(\mathrm{~m}, 1 \mathrm{H}), 0.89(\mathrm{~d}, J=$ $6.8 \mathrm{~Hz}, 3 \mathrm{H}), 0.73(\mathrm{~d}, J=6.9 \mathrm{~Hz}, 3 \mathrm{H}) 0.73(\mathrm{~d}, J=6.9 \mathrm{~Hz}, 3 \mathrm{H}) \mathrm{ppm} ;{ }^{13} \mathrm{C} \mathrm{NMR}\left(101 \mathrm{MHz}, \mathrm{CDCl}_{3}\right) \delta=$ 211.0, 163.2, 136.4 129.6, 119.2, 118.9, 118.8, 47.4, 43.7, 40.2, 34.6, 32.7, 28.9, 24.1, 22.3, 21.5, 16.6 ppm; HRMS (ESI): calcd. for $\mathrm{C}_{17} \mathrm{H}_{25} \mathrm{O}_{2}[\mathrm{M}+\mathrm{H}]^{+}: 261.1849$ found: 261.1852 .

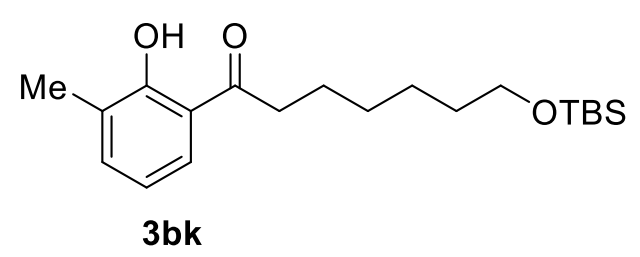

7-((tert-Butyldimethylsilyl)oxy)-1-(2-hydroxy-3-methylphenyl)heptan-1-one (3bk) was isolated through column chromatography on silica gel (petroleum ether: $\mathrm{EtOAc}=20: 1)$ as a colorless oil $(30 \mathrm{mg}, 43 \%$ yield $) .{ }^{1} \mathrm{H} \mathrm{NMR}(400 \mathrm{MHz}$, $\left.\mathrm{CDCl}_{3}\right) \delta=12.71(\mathrm{~s}, 1 \mathrm{H}), 7.65-7.55(\mathrm{~m}, 1 \mathrm{H}), 7.33(\mathrm{~d}, J=7.3 \mathrm{~Hz}, 1 \mathrm{H}), 6.79(\mathrm{t}, J$ $=7.7 \mathrm{~Hz}, 1 \mathrm{H}), 3.61(\mathrm{t}, J=6.4 \mathrm{~Hz}, 2 \mathrm{H}), 2.98(\mathrm{t}, J=7.5 \mathrm{~Hz}, 2 \mathrm{H}), 2.26(\mathrm{~s}, 3 \mathrm{H})$, $1.84-1.69(\mathrm{~m}, 2 \mathrm{H}), 1.59-1.48(\mathrm{~m}, 2 \mathrm{H}), 1.44-1.35(\mathrm{~m}, 4 \mathrm{H}), 0.89(\mathrm{~s}, 9 \mathrm{H}), 0.05(\mathrm{~s}$, 6H) ppm; ${ }^{13} \mathrm{C} \mathrm{NMR}\left(101 \mathrm{MHz}, \mathrm{CDCl}_{3}\right) \delta=207.1,161.0,136.9,127.56,127.55,118.6,118.1,63.1,38.4,32.7,29.1,26.0$ (3C), 25.7, 24.7, 18.9, 15.54, -5.3 (2C) ppm; HRMS (ESI): calcd. for $\mathrm{C}_{20} \mathrm{H}_{35} \mathrm{O}_{3} \mathrm{Si}[\mathrm{M}+\mathrm{H}]^{+}: 351.2350$ found: 351.2352 .

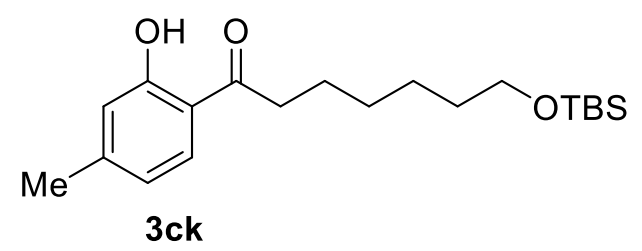

0.05 (s, 6H) ppm; ${ }^{13} \mathrm{C}$ NMR $\left(101 \mathrm{MHz}, \mathrm{CDCl}_{3}\right) \delta=206.3,162.7,147.8,129.9,120.1,118.5,117.1,63.1,38.1,32.7,29.1$, 26.0 (3C), 25.7, 24.7, 21.9, 18.4, -5.3 (2C) ppm; HRMS (ESI): calcd. for $\mathrm{C}_{20} \mathrm{H}_{35} \mathrm{O}_{3} \mathrm{Si}[\mathrm{M}+\mathrm{H}]^{+}: 351.2350$ found: 351.2342.

7-((tert-Butyldimethylsilyl)oxy)-1-(2-hydroxy-4-methylphenyl)heptan-1-one (3ck) was isolated through column chromatography on silica gel(petroleum ether: $\mathrm{EtOAc}=20: 1)$ as a colorless oil $(56 \mathrm{mg}, 80 \%$ yield $) .{ }^{1} \mathrm{H}$ NMR $(400 \mathrm{MHz}$, $\left.\mathrm{CDCl}_{3}\right) \delta=12.43(\mathrm{~s}, 1 \mathrm{H}), 7.63(\mathrm{~d}, J=8.2 \mathrm{~Hz}, 1 \mathrm{H}), 6.78(\mathrm{~d}, J=1.5 \mathrm{~Hz}, 1 \mathrm{H}), 6.70$ (dd, $J=8.2,1.7 \mathrm{~Hz}, 1 \mathrm{H}), 3.61(\mathrm{t}, J=6.4 \mathrm{~Hz}, 2 \mathrm{H}), 2.94(\mathrm{t}, J=7.5 \mathrm{~Hz}, 2 \mathrm{H}), 2.34$ (s, 3H), 1.80-1.64 (m, 2H), 1.62-1.47 (m, 2H), 1.46-1.31 (m, 4H), $0.89(\mathrm{~s}, 9 \mathrm{H})$, 


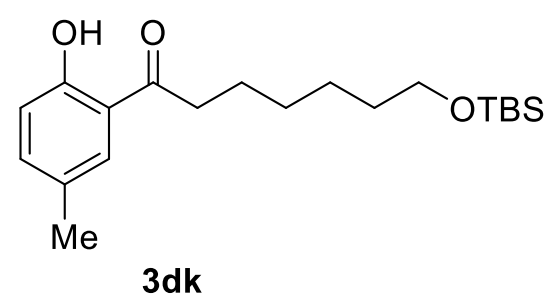

$3 \mathbf{d k}$

was isolated through column chromatography on silica gel (petroleum ether: EtOAc= $20: 1)$ as a colorless oil $\left(68 \mathrm{mg}, 97 \%\right.$ yield). ${ }^{1} \mathrm{H}$ NMR $\left(400 \mathrm{MHz}, \mathrm{CDCl}_{3}\right) \delta=12.22(\mathrm{~s}$, $1 \mathrm{H}), 7.63-7.49(\mathrm{~m}, 1 \mathrm{H}), 7.31-7.23(\mathrm{~m}, 1 \mathrm{H}), 6.88(\mathrm{~d}, J=8.4 \mathrm{~Hz}, 1 \mathrm{H}), 3.61(\mathrm{t}, J=6.5$ $\mathrm{Hz}, 2 \mathrm{H}), 2.97$ (t, $J=7.4 \mathrm{~Hz}, 2 \mathrm{H}), 2.31(\mathrm{~s}, 3 \mathrm{H}), 1.84-1.68(\mathrm{~m}, 2 \mathrm{H}), 1.66-1.50(\mathrm{~m}, 2 \mathrm{H})$, $1.46-1.28(\mathrm{~m}, 4 \mathrm{H}), 0.90(\mathrm{~s}, 9 \mathrm{H}), 0.05(\mathrm{~s}, 6 \mathrm{H}) \mathrm{ppm} ;{ }^{13} \mathrm{C} \mathrm{NMR}\left(101 \mathrm{MHz}, \mathrm{CDCl}_{3}\right) \delta=$ 206.7, 160.4, 137.2, 129.7, 127.9, 119.0, 118.3, 63.1, 38.2, 32.7, 29.1, 26.0, 25.7, 24.4, 20.6 (3C), 18.4, -5.6 (2C) ppm; HRMS (ESI): calcd. for $\mathrm{C}_{20} \mathrm{H}_{35} \mathrm{O}_{3} \mathrm{Si}[\mathrm{M}+\mathrm{H}]^{+}: 351.2350$ found: 351.2356 .

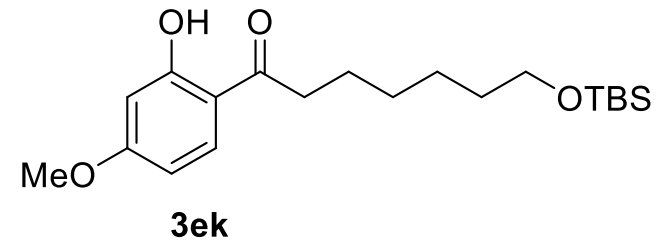

$\left.\mathrm{MHz}, \mathrm{CDCl}_{3}\right) \delta=205.1,165.9,165.4,131.6,113.5,107.6,100.9,63.1,55.6,38.0,32.7$
(2C) ppm; HRMS (ESI): calcd. for $\mathrm{C}_{20} \mathrm{H}_{35} \mathrm{O}_{4} \mathrm{Si}[\mathrm{M}+\mathrm{H}]^{+}: 367.2299$ found: 367.2300 .

7-((tert-Butyldimethylsilyl)oxy)-1-(2-hydroxy-4-methoxyphenyl)heptan-1-one (3ek) was isolated through column chromatography on silica gel (petroleum ether: $\mathrm{EtOAc}=10: 1)$ as a colorless oil $(31 \mathrm{mg}, 42 \%$ yield $) .{ }^{1} \mathrm{H}$ NMR $(400 \mathrm{MHz}$, $\left.\mathrm{CDCl}_{3}\right) \delta=12.90(\mathrm{~s}, 1 \mathrm{H}), 7.80-7.59(\mathrm{~m}, 1 \mathrm{H}), 6.56-6.30(\mathrm{~m}, 2 \mathrm{H}), 3.84(\mathrm{~s}, 3 \mathrm{H})$, $3.61(\mathrm{t}, J=6.5 \mathrm{~Hz}, 2 \mathrm{H}), 2.90$ (t, $J=7.5 \mathrm{~Hz}, 2 \mathrm{H}), 1.80-1.67(\mathrm{~m}, 2 \mathrm{H}), 1.57-1.46$ $(\mathrm{m}, 2 \mathrm{H}), 1.44-1.35(\mathrm{~m}, 4 \mathrm{H}), 0.89(\mathrm{~s}, 9 \mathrm{H}), 0.05(\mathrm{~s}, 6 \mathrm{H}) \mathrm{ppm} ;{ }^{13} \mathrm{C}$ NMR $(101$

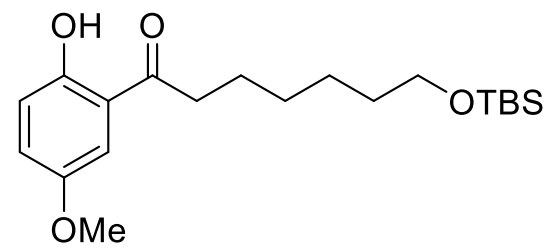

3fk

7-((tert-Butyldimethylsilyl)oxy)-1-(2-hydroxy-5-methoxyphenyl)heptan-1-one

(3fk) was isolated through column chromatography on silica gel (petroleum ether: EtOAc= $10: 1)$ as a colorless oil (54 mg, $74 \%$ yield). $\left.{ }^{1} \mathrm{H} \mathrm{NMR} \mathrm{(400} \mathrm{MHz,} \mathrm{CDCl}_{3}\right) \delta=11.98$ (s, $1 \mathrm{H}), 7.20(\mathrm{~d}, J=3.0 \mathrm{~Hz}, 1 \mathrm{H}), 7.10(\mathrm{dd}, J=9.1,3.1 \mathrm{~Hz}, 1 \mathrm{H}), 6.92(\mathrm{~d}, J=9.0 \mathrm{~Hz}, 1 \mathrm{H})$, $3.80(\mathrm{~s}, 3 \mathrm{H}), 3.61(\mathrm{t}, J=6.5 \mathrm{~Hz}, 2 \mathrm{H}), 2.96(\mathrm{t}, J=7.4 \mathrm{~Hz}, 2 \mathrm{H}), 1.81-1.70(\mathrm{~m}, 2 \mathrm{H}), 1.63-$ $1.48(\mathrm{~m}, 2 \mathrm{H}), 1.48-1.35(\mathrm{~m}, 4 \mathrm{H}), 0.89(\mathrm{~s}, 9 \mathrm{H}), 0.05(\mathrm{~s}, 6 \mathrm{H}) \mathrm{ppm} ;{ }^{13} \mathrm{C} \mathrm{NMR}(101 \mathrm{MHz}$, $\left.\mathrm{CDCl}_{3}\right) \delta=206.3,156.8,151.6,123.8,119.3,118.8,112.9,63.1,56.0,38.3,32.7,29.1,26.0(3 \mathrm{C}), 25.7,24.3,18.4,-5.3(2 \mathrm{C})$ ppm. HRMS (ESI): calcd. for $\mathrm{C}_{20} \mathrm{H}_{35} \mathrm{O}_{4} \mathrm{Si}[\mathrm{M}+\mathrm{H}]^{+}: 367.2299$ found: 367.2303 .

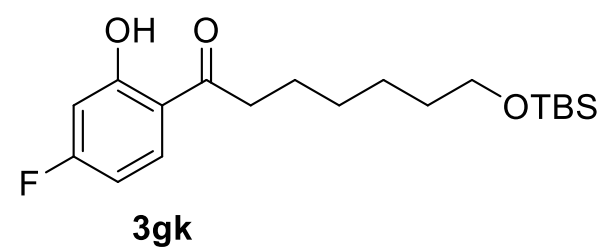

7-((tert-Butyldimethylsilyl)oxy)-1-(4-fluoro-2-hydroxyphenyl)heptan-1-one (3gk) was isolated through column chromatography on silica gel (petroleum ether: EtOAc $=20: 1)$ as a colorless oil $(63 \mathrm{mg}, 89 \%$ yield $) .{ }^{1} \mathrm{H} \mathrm{NMR}\left(400 \mathrm{MHz}, \mathrm{CDCl}_{3}\right)$ $\delta=12.73(\mathrm{~d}, J=1.5 \mathrm{~Hz}, 1 \mathrm{H}), 7.82-7.70(\mathrm{~m}, 1 \mathrm{H}), 6.71-6.52(\mathrm{~m}, 2 \mathrm{H}), 3.61(\mathrm{t}, J=$ $6.5 \mathrm{~Hz}, 2 \mathrm{H}), 2.94(\mathrm{t}, J=7.4 \mathrm{~Hz}, 2 \mathrm{H}), 1.80-1.67(\mathrm{~m}, 2 \mathrm{H}), 1.57-1.49(\mathrm{~m}, 2 \mathrm{H}), 1.45-$ $1.33(\mathrm{~m}, 4 \mathrm{H}), 0.89(\mathrm{~s}, 9 \mathrm{H}), 0.05(\mathrm{~s}, 6 \mathrm{H}) \mathrm{ppm} ;{ }^{13} \mathrm{C} \mathrm{NMR}\left(101 \mathrm{MHz}, \mathrm{CDCl}_{3}\right) \delta=$ $205.7,168.5\left(\mathrm{C}-\mathrm{F},{ }^{1} J_{\mathrm{C}-\mathrm{F}}=256.3 \mathrm{~Hz}\right), 166.0\left(\mathrm{C}-\mathrm{F},{ }^{1} J_{\mathrm{C}-\mathrm{F}}=256.3 \mathrm{~Hz}\right), 165.2\left(\mathrm{C}-\mathrm{F},{ }^{3} J_{\mathrm{C}-\mathrm{F}}=14.3 \mathrm{~Hz}\right), 165.0\left(\mathrm{C}-\mathrm{F},{ }^{3} J \mathrm{C}-\mathrm{F}=14.3\right.$ $\mathrm{Hz}), 132.4\left(\mathrm{C}-\mathrm{F},{ }^{3} J_{\mathrm{C}-\mathrm{F}}=11.8 \mathrm{~Hz}\right), 132.3\left(\mathrm{C}-\mathrm{F},{ }^{3} J_{\mathrm{C}-\mathrm{F}}=11.8 \mathrm{~Hz}\right), 116.50\left(\mathrm{C}-\mathrm{F},{ }^{4} J_{\mathrm{C}-\mathrm{F}}=2.3 \mathrm{~Hz}\right), 116.47\left(\mathrm{C}-\mathrm{F},{ }^{4} J \mathrm{C}-\mathrm{F}=2.3 \mathrm{~Hz}\right)$, $107.2\left(\mathrm{C}-\mathrm{F},{ }^{2} J_{\mathrm{C}-\mathrm{F}}=22.7 \mathrm{~Hz}\right), 107.0\left(\mathrm{C}-\mathrm{F},{ }^{2} J_{\mathrm{C}-\mathrm{F}}=22.7 \mathrm{~Hz}\right), 105.2\left(\mathrm{C}-\mathrm{F},{ }^{2} J_{\mathrm{C}-\mathrm{F}}=23.5 \mathrm{~Hz}\right), 104.9\left(\mathrm{C}-\mathrm{F},{ }^{2} J_{\mathrm{C}-\mathrm{F}}=23.5 \mathrm{~Hz}\right), 63.1$, 38.3, 32.6, 29.1 , 26.0 (3C), 25.7 , 24.5 , 18.4 , -5.7 (2C) ppm; ${ }^{19} \mathrm{~F} \mathrm{NMR}\left(376 \mathrm{MHz}, \mathrm{CDCl}_{3}\right) \delta=-93.83--108.03(\mathrm{~m}, 1 \mathrm{~F}) \mathrm{ppm}$; HRMS (ESI): calcd. for $\mathrm{C}_{19} \mathrm{H}_{32} \mathrm{FO}_{3} \mathrm{Si}[\mathrm{M}+\mathrm{H}]^{+}: 355.2099$ found:355.2105

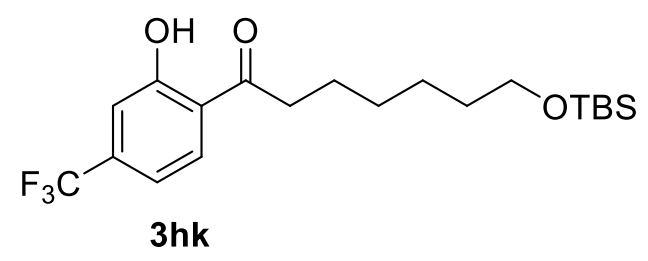

7-((tert-Butyldimethylsilyl)oxy)-1-(2-hydroxy-4(trifluoromethyl)phenyl)heptan-

1-one (3hk) was isolated through column chromatography on silica gel (petroleum ether: $\mathrm{EtOAc}=20: 1)$ as a colorless oil $\left(65 \mathrm{mg}, 80 \%\right.$ yield). ${ }^{1} \mathrm{H}$ NMR $\left(400 \mathrm{MHz}, \mathrm{CDCl}_{3}\right) \delta=12.42(\mathrm{~s}, 1 \mathrm{H}), 7.88(\mathrm{~d}, J=8.3 \mathrm{~Hz}, 1 \mathrm{H}), 7.38-7.19(\mathrm{~m}$, 1H), $7.13(\mathrm{dd}, J=8.4,1.7 \mathrm{~Hz}, 1 \mathrm{H}), 3.62$ (t, $J=6.4 \mathrm{~Hz}, 2 \mathrm{H}), 3.02(\mathrm{t}, J=7.4 \mathrm{~Hz}$, $2 \mathrm{H}), 1.84-1.72(\mathrm{~m}, 2 \mathrm{H}), 1.59-1.50(\mathrm{~m}, 2 \mathrm{H}), 1.47-1.35(\mathrm{~m}, 4 \mathrm{H}), 0.89(\mathrm{~s}, 9 \mathrm{H})$, $0.05(\mathrm{~s}, 6 \mathrm{H}) \mathrm{ppm} ;{ }^{13} \mathrm{C} \mathrm{NMR}\left(101 \mathrm{MHz}, \mathrm{CDCl}_{3}\right) \delta=206.6,162.3,137.6\left(\mathrm{C}-\mathrm{F},{ }^{2} J_{\mathrm{C}-\mathrm{F}}=33.0 \mathrm{~Hz}\right), 137.3\left(\mathrm{C}-\mathrm{F},{ }^{2} J \mathrm{C}-\mathrm{F}=33.0 \mathrm{~Hz}\right)$, 
$136.9\left(\mathrm{C}-\mathrm{F},{ }^{2} J_{\mathrm{C}-\mathrm{F}}=33.0 \mathrm{~Hz}\right), 136.6\left(\mathrm{C}-\mathrm{F},{ }^{2} J_{\mathrm{C}-\mathrm{F}}=33.0 \mathrm{~Hz}\right), 130.7,127.1\left(\mathrm{C}-\mathrm{F},{ }^{1} J_{\mathrm{C}-\mathrm{F}}=273.7 \mathrm{~Hz}\right), 124.4\left(\mathrm{C}-\mathrm{F},{ }^{1} J_{\mathrm{C}-\mathrm{F}}=273.7\right.$ $\mathrm{Hz}), 121.7\left(\mathrm{C}-\mathrm{F},{ }^{1} J_{\mathrm{C}-\mathrm{F}}=273.7 \mathrm{~Hz}\right), 121.2,119.0\left(\mathrm{C}-\mathrm{F},{ }^{1} J_{\mathrm{C}-\mathrm{F}}=273.7 \mathrm{~Hz}\right), 116.10\left(\mathrm{C}-\mathrm{F},{ }^{3} J_{\mathrm{C}-\mathrm{F}}=4.0 \mathrm{~Hz}\right), 116.06\left(\mathrm{C}-\mathrm{F},{ }^{3} J_{\mathrm{C}-\mathrm{F}}=\right.$ $4.0 \mathrm{~Hz}), 116.02\left(\mathrm{C}-\mathrm{F},{ }^{3} J_{\mathrm{C}-\mathrm{F}}=4.0 \mathrm{~Hz}\right), 115.98\left(\mathrm{C}-\mathrm{F},{ }^{3} J_{\mathrm{C}-\mathrm{F}}=4.0 \mathrm{~Hz}\right), 115.24\left(\mathrm{C}-\mathrm{F},{ }^{3} J_{\mathrm{C}-\mathrm{F}}=3.6 \mathrm{~Hz}\right), 115.21\left(\mathrm{C}-\mathrm{F},{ }^{3} J_{\mathrm{C}-\mathrm{F}}=3.6\right.$ $\mathrm{Hz}), 115.21\left(\mathrm{C}-\mathrm{F},{ }^{3} J_{\mathrm{C}-\mathrm{F}}=3.6 \mathrm{~Hz}\right), 115.17\left(\mathrm{C}-\mathrm{F},{ }^{3} J_{\mathrm{C}-\mathrm{F}}=3.6 \mathrm{~Hz}\right), 63.1,38.6,32.6,29.0,26.0(3 \mathrm{C}), 25.6,24.2,18.4,-5.3(2 \mathrm{C})$ ppm; ${ }^{19} \mathrm{~F}$ NMR (376 MHz, $\mathrm{CDCl}_{3}$ ) $\delta=-63.91$ (s, 3F) ppm. HRMS (ESI): calcd. for $\mathrm{C}_{20} \mathrm{H}_{32} \mathrm{FO}_{3} \mathrm{Si}[\mathrm{M}+\mathrm{H}]^{+}: 405.2067$ found: 405.2068

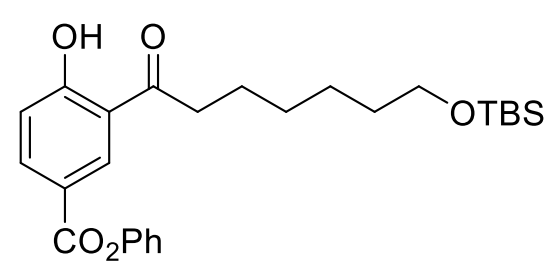

3ik

Phenyl 3-(7-((tert-butyldimethylsilyl)oxy)heptanoyl)-4-hydroxybenzoate (3ik) was isolated through column chromatography on silica gel (petroleum ether: EtOAc $=20: 1$ ) as a colorless oil (65 mg, $71 \%$ yield). ${ }^{1} \mathrm{H}$ NMR $\left(500 \mathrm{MHz}, \mathrm{CDCl}_{3}\right) \delta=12.92(\mathrm{~s}, 1 \mathrm{H})$, $8.66(\mathrm{t}, J=2.0 \mathrm{~Hz}, 1 \mathrm{H}), 8.36-8.20(\mathrm{~m}, 1 \mathrm{H}), 7.50-7.40(\mathrm{~m}, 2 \mathrm{H}), 7.35-7.18(\mathrm{~m}, 3 \mathrm{H})$, $7.13-7.03(\mathrm{~m}, 1 \mathrm{H}), 3.61(\mathrm{t}, J=6.5 \mathrm{~Hz}, 2 \mathrm{H}), 3.09(\mathrm{t}, J=7.4 \mathrm{~Hz}, 2 \mathrm{H}), 1.85-1.73(\mathrm{~m}$, $2 \mathrm{H}), 1.60-1.51(\mathrm{~m}, 2 \mathrm{H}), 1.49-1.37(\mathrm{~m}, 4 \mathrm{H}), 0.88(\mathrm{~s}, 9 \mathrm{H}), 0.04(\mathrm{~s}, 6 \mathrm{H}) \mathrm{ppm} ;{ }^{13} \mathrm{C} \mathrm{NMR}$ $\left(126 \mathrm{MHz}, \mathrm{CDCl}_{3}\right) \delta=206.9,166.7,164.2,150.9,137.4,133.2,129.6$ (2C), 126.0, 121.7 (2C), 120.3, 119.04, 118.97, 63.1, 38.3, 32.7, 29.0, 26.0 (3C), 25.7, 24.2, 18.4, -5.3 (2C) ppm. HRMS (ESI): calcd. for $\mathrm{C}_{26} \mathrm{H}_{37} \mathrm{O}_{5} \mathrm{Si}[\mathrm{M}+\mathrm{H}]^{+}: 457.2405$ found: 457.2414

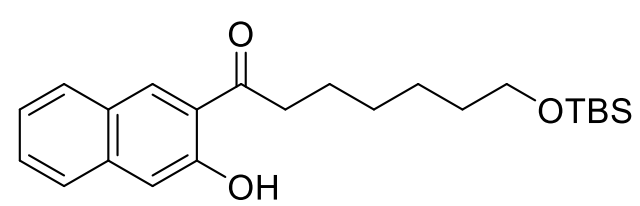

$3 \mathbf{j k}$

7-((tert-Butyldimethylsilyl)oxy)-1-(3-hydroxynaphthalen-2-yl)heptan-1-one (3jk) was isolated through column chromatography on silica gel (petroleum ether: $\mathrm{EtOAc}=20: 1)$ as a white solid (46 mg, $60 \%$ yield). ${ }^{1} \mathrm{H}$ NMR (400 MHz, $\left.\mathrm{CDCl}_{3}\right) \delta=11.70(\mathrm{~s}, 1 \mathrm{H}), 8.37(\mathrm{~s}, 1 \mathrm{H}), 7.81(\mathrm{~d}, J=8.3 \mathrm{~Hz}, 1 \mathrm{H}), 7.67(\mathrm{~d}, J=8.4$ $\mathrm{Hz}, 1 \mathrm{H}), 7.51(\mathrm{t}, J=7.6 \mathrm{~Hz}, 1 \mathrm{H}), 7.33$ (t, $J=7.6 \mathrm{~Hz}, 1 \mathrm{H}), 7.27$ (d, $J=8.5 \mathrm{~Hz}$, $1 \mathrm{H}), 3.62(\mathrm{t}, J=6.4 \mathrm{~Hz}, 2 \mathrm{H}), 3.17(\mathrm{t}, J=7.4 \mathrm{~Hz}, 2 \mathrm{H}), 1.88-1.76(\mathrm{~m}, 2 \mathrm{H}), 1.64-$ $1.50(\mathrm{~m}, 2 \mathrm{H}), 1.52-1.39(\mathrm{~m}, 4 \mathrm{H}), 0.90(\mathrm{~s}, 9 \mathrm{H}), 0.05(\mathrm{~s}, 6 \mathrm{H}) \mathrm{ppm} ;{ }^{13} \mathrm{C} \mathrm{NMR}\left(101 \mathrm{MHz}, \mathrm{CDCl}_{3}\right) \delta=207.1,157.2,138.1,132.7$, 129.6, 129.4, 126.8, 126.2, 124.0, 121.0, 112.3, 63.1, 38.5, 32.7, 29.1, 26.0 (3C), 25.7, 24.6, 18.4, -5.2 (2C) ppm; HRMS (ESI): calcd. for $\mathrm{C}_{23} \mathrm{H}_{35} \mathrm{O}_{3} \mathrm{Si}[\mathrm{M}+\mathrm{H}]^{+}: 387.2350$ found: 387.2357 .

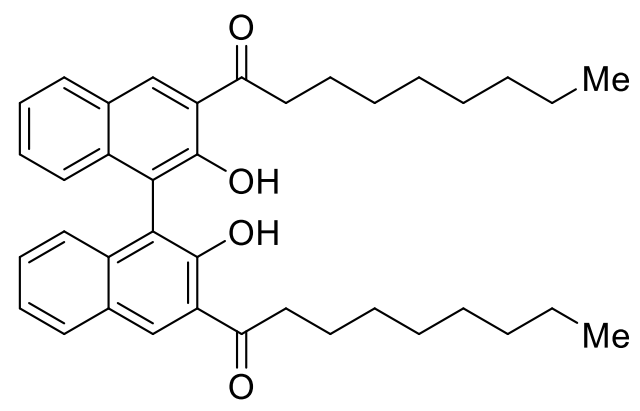

$3 \mathbf{k b}$

\section{1,1'-(2,2'-Dihydroxy-[1,1'-binaphthalene]-3,3'-diyl)bis(nonan-1-one)}

(3kb) was isolated through column chromatography on silica gel (petroleum ether: EtOAc $=20: 1)$ as a yellow solid (55 mg, $48 \%$ yield). ${ }^{1} \mathrm{H}$ NMR $\left(500 \mathrm{MHz}, \mathrm{CDCl}_{3}\right)$ $\delta=11.70(\mathrm{~s}, 2 \mathrm{H}), 8.35(\mathrm{~s}, 2 \mathrm{H}), 7.79(\mathrm{~d}, J=8.2 \mathrm{~Hz}, 2 \mathrm{H}), 7.65(\mathrm{~d}, J=8.3 \mathrm{~Hz}, 2 \mathrm{H})$, 7.49 (t, $J=7.6 \mathrm{~Hz}, 2 \mathrm{H}), 7.31(\mathrm{t}, J=7.6 \mathrm{~Hz}, 2 \mathrm{H}), 3.13(\mathrm{t}, J=7.5 \mathrm{~Hz}, 4 \mathrm{H}), 1.83$ $1.72(\mathrm{~m}, 4 \mathrm{H}), 1.46-1.39(\mathrm{~m}, 4 \mathrm{H}), 1.37-1.24(\mathrm{~m}, 16 \mathrm{H}), 0.89(\mathrm{t}, J=6.6 \mathrm{~Hz}, 6 \mathrm{H})$ ppm; ${ }^{13} \mathrm{C} \mathrm{NMR}\left(126 \mathrm{MHz}, \mathrm{CDCl}_{3}\right) \delta=207.2(2 \mathrm{C}), 157.3(2 \mathrm{C}), 138.1$ (2C), 132.7 (2C), 129.6 (2C), 129.4 (2C), 126.8 (2C), 126.2 (2C), 124.0 (2C), 121.0 (2C), 112.3 (2C), 38.5 (2C), 31.9 (2C), 29.5 (2C), 29.4 (2C), 29.2 (2C), 24.6 (2C), 22.7 (2C), 14.1 (2C) ppm; HRMS (ESI): calcd. for $\mathrm{C}_{38} \mathrm{H}_{47} \mathrm{O}_{4}[\mathrm{M}+\mathrm{H}]^{+}: 567.3469$

found: 567.3469 .

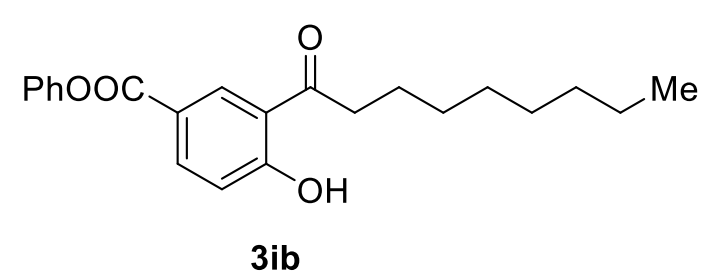

Phenyl 4-hydroxy-3-nonanoylbenzoate (3ib) was isolated through column chromatography on silica gel (petroleum ether: EtOAc $=20: 1$ ) as a colorless oil (87 mg, $86 \%$ yield). ${ }^{1} \mathrm{H}$ NMR (500 MHz, $\left.\mathrm{CDCl}_{3}\right) \delta=12.93(\mathrm{~s}, 1 \mathrm{H}), 8.65$ $(\mathrm{d}, J=2.1 \mathrm{~Hz}, 1 \mathrm{H}), 8.28(\mathrm{dd}, J=8.8,2.2 \mathrm{~Hz}, 1 \mathrm{H}), 7.44(\mathrm{t}, J=7.9 \mathrm{~Hz}, 2 \mathrm{H})$, $7.28(\mathrm{t}, J=7.5 \mathrm{~Hz}, 1 \mathrm{H}), 7.21(\mathrm{dd}, J=7.4,1.6 \mathrm{~Hz}, 2 \mathrm{H}), 7.07(\mathrm{~d}, J=8.8 \mathrm{~Hz}$, $1 \mathrm{H}), 3.08$ (t, $J=7.4 \mathrm{~Hz}, 2 \mathrm{H}), 1.85-1.70(\mathrm{~m}, 2 \mathrm{H}), 1.45-1.37$ (m, 2H), 1.39$1.22(\mathrm{~m}, 8 \mathrm{H}), 0.88(\mathrm{t}, J=6.8 \mathrm{~Hz}, 3 \mathrm{H}) \mathrm{ppm} ;{ }^{13} \mathrm{C} \mathrm{NMR}\left(126 \mathrm{MHz}, \mathrm{CDCl}_{3}\right) \delta=207.0,166.7,164.1,150.8,137.4,133.2,129.5$ (2C), 126.0, 121.7 (2C), 120.3, 119.00, 118.95, 38.4, 31.8, 29.4, 29.2, 29.1, 24.2, 22.7, 14.1 ppm; HRMS (ESI): calcd. for 


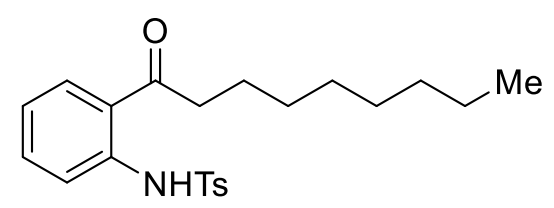

$5 \mathrm{ab}$

4-Methyl-N-(2-nonanoylphenyl)benzenesulfonamide (5ab) was isolated through column chromatography on silica gel (petroleum ether: EtOAc $=10: 1$ ) as a white solid (40 mg, $52 \%$ yield). ${ }^{1} \mathrm{H}$ NMR $\left(400 \mathrm{MHz}, \mathrm{CDCl}_{3}\right) \delta=11.45(\mathrm{~s}, 1 \mathrm{H}), 7.80(\mathrm{dd}, J=7.9$, $1.5 \mathrm{~Hz}, 1 \mathrm{H}), 7.75-7.66(\mathrm{~m}, 3 \mathrm{H}), 7.49-7.38(\mathrm{~m}, 1 \mathrm{H}), 7.21(\mathrm{~d}, J=8.0 \mathrm{~Hz}, 2 \mathrm{H}), 7.06(\mathrm{t}, J$ $=7.5 \mathrm{~Hz}, 1 \mathrm{H}), 2.87(\mathrm{t}, J=7.4 \mathrm{~Hz}, 2 \mathrm{H}), 2.36(\mathrm{~s}, 3 \mathrm{H}), 1.65-1.52(\mathrm{~m}, 2 \mathrm{H}), 1.36-1.25(\mathrm{~m}$, $10 \mathrm{H}), 0.89$ (t, $J=6.8 \mathrm{~Hz}, 3 \mathrm{H}) \mathrm{ppm} ;{ }^{13} \mathrm{C} \mathrm{NMR}\left(101 \mathrm{MHz}, \mathrm{CDCl}_{3}\right) \delta=204.8,143.7$, 139.9, 136.6, 134.5, 131.0, 129.6 (2C), 127.2 (2C), 122.7, 122.5, 119.6, 39.7, 31.8, 29.4, 29.21, 29.15, 24.5, 22.7, 21.5, 14.1 ppm; HRMS (ESI): calcd. for $\mathrm{C}_{22} \mathrm{H}_{30} \mathrm{NO}_{3} \mathrm{~S}[\mathrm{M}+\mathrm{H}]^{+}: 388.1941$ found: 388.1945 .<smiles>O=C(CCl)c1ccccc1NC(F)(F)F</smiles>

5 ac

$\mathrm{N}$-(2-(2-cyclohexylacetyl)phenyl)-4-methylbenzenesulfonamide (5ac) was isolated through column chromatography on silica gel (petroleum ether: $\mathrm{EtOAc}=10: 1)$ as a white solid (38 $\mathrm{mg}, 51 \%$ yield). ${ }^{1} \mathrm{H}$ NMR $\left(400 \mathrm{MHz}, \mathrm{CDCl}_{3}\right) \delta=11.51(\mathrm{~s}, 1 \mathrm{H}), 7.79(\mathrm{dd}, J=8.0,1.5 \mathrm{~Hz}, 1 \mathrm{H}), 7.76-7.68(\mathrm{~m}, 3 \mathrm{H}), 7.49-7.40$ $(\mathrm{m}, 1 \mathrm{H}), 7.20(\mathrm{~d}, J=8.1 \mathrm{~Hz}, 2 \mathrm{H}), 7.11-7.02(\mathrm{~m}, 1 \mathrm{H}), 2.73(\mathrm{~d}, J=6.8 \mathrm{~Hz}, 2 \mathrm{H}), 2.35(\mathrm{~s}, 3 \mathrm{H}), 1.91-1.77(\mathrm{~m}$, $1 \mathrm{H}), 1.73-1.56(\mathrm{~m}, 4 \mathrm{H}), 1.30-1.10(\mathrm{~m}, 4 \mathrm{H}), 1.01-0.85(\mathrm{~m}, 2 \mathrm{H}) \mathrm{ppm} ;{ }^{13} \mathrm{C} \mathrm{NMR}\left(101 \mathrm{MHz}, \mathrm{CDCl}_{3}\right) \delta=$ 204.5, 143.7, 140.1, 136.7, 134.6, 131.2, 129.6 (2C), 127.2 (2C), 122.7, 122.6, 119.6, 47.3, 34.8, 33.3 (2C), 26.2, 26.1 (2C), 21.5 ppm; HRMS (ESI): calcd. for $\mathrm{C}_{21} \mathrm{H}_{25} \mathrm{NO}_{3} \mathrm{SNa}[\mathrm{M}+\mathrm{Na}]^{+}$: 394.1447 found: 394.1445 .

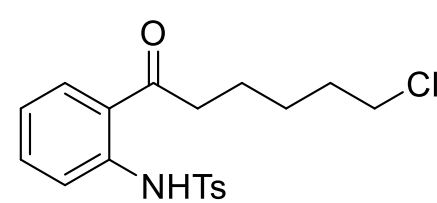

5ai

$N-(2-(6-c h l o r o h e x a n o y l)$ phenyl)-4-methylbenzenesulfonamide (5ai) was isolated through column chromatography on silica gel (petroleum ether: $\mathrm{EtOAc}=10: 1)$ as a white solid (46 mg, $61 \%$ yield). ${ }^{1} \mathrm{H}$ NMR $\left(500 \mathrm{MHz}, \mathrm{CDCl}_{3}\right) \delta=11.41(\mathrm{~s}, 1 \mathrm{H}), 7.80(\mathrm{dd}, J=8.1,1.5 \mathrm{~Hz}, 1 \mathrm{H})$, $7.73(\mathrm{~s}, 1 \mathrm{H}), 7.73-7.67(\mathrm{~m}, 2 \mathrm{H}), 7.49-7.41(\mathrm{~m}, 1 \mathrm{H}), 7.22(\mathrm{~d}, J=8.0 \mathrm{~Hz}, 2 \mathrm{H}), 7.06(\mathrm{t}, J=7.7$ $\mathrm{Hz}, 1 \mathrm{H}), 3.56(\mathrm{t}, J=6.6 \mathrm{~Hz}, 2 \mathrm{H}), 2.91(\mathrm{t}, J=7.3 \mathrm{~Hz}, 2 \mathrm{H}), 2.36(\mathrm{~s}, 3 \mathrm{H}), 1.85-1.75(\mathrm{~m}, 2 \mathrm{H})$, 1.69-1.62 (m, 2H), 1.53-1.44 (m, 2H) ppm; $\left.{ }^{13} \mathrm{C} \mathrm{NMR} \mathrm{(126} \mathrm{MHz,} \mathrm{CDCl}_{3}\right) \delta=204.1,143.8$, 140.0, 136.7, 134.7, 130.9, 129.6 (2C), 127.3 (2C), 122.7, 122.4, 119.6, 44.8, 39.4, 32.4, 26.4, 23.5, 21.5 ppm; HRMS (ESI): calcd. for $\mathrm{C}_{19} \mathrm{H}_{22} \mathrm{ClNO}_{3} \mathrm{SNa}[\mathrm{M}+\mathrm{Na}]^{+}: 402.0901$ found: 402.0904

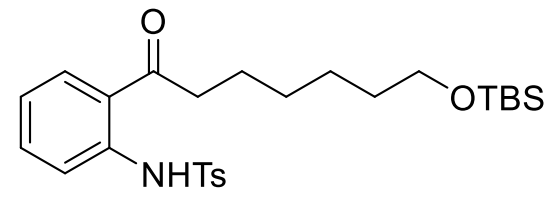

5ak

$N-(2-(7-(($ tert-butyldimethylsilyl)oxy)heptanoyl)phenyl)-4-methylbenzenesulfonamide (5ak) was isolated through column chromatography on silica gel (petroleum ether: EtOAc $=10: 1)$ as a white solid $(55 \mathrm{mg}, 56 \%$ yield $) .{ }^{1} \mathrm{H} \mathrm{NMR}\left(400 \mathrm{MHz}, \mathrm{CDCl}_{3}\right) \delta=$ $11.45(\mathrm{~s}, 1 \mathrm{H}), 7.80(\mathrm{dd}, J=8.1,1.5 \mathrm{~Hz}, 1 \mathrm{H}), 7.75-7.65(\mathrm{~m}, 3 \mathrm{H}), 7.49-7.39(\mathrm{~m}, 1 \mathrm{H})$, 7.25- $7.17(\mathrm{~m}, 2 \mathrm{H}), 7.11-7.02(\mathrm{~m}, 1 \mathrm{H}), 3.61(\mathrm{t}, J=6.5 \mathrm{~Hz}, 2 \mathrm{H}), 2.87(\mathrm{t}, J=7.4 \mathrm{~Hz}$, $2 \mathrm{H}), 2.35(\mathrm{~s}, 3 \mathrm{H}), 1.68-1.58(\mathrm{~m}, 2 \mathrm{H}), 1.57-1.47(\mathrm{~m}, 2 \mathrm{H}), 1.38-1.31(\mathrm{~m}, 4 \mathrm{H}), 0.90(\mathrm{~s}$, 9H), 0.06 (s, 6H) ppm; ${ }^{13} \mathrm{C}$ NMR (101 MHz, $\left.\mathrm{CDCl}_{3}\right) \delta=204.7,143.8,140.0,136.6,134.6,131.0,129.6$ (2C), 127.3 (2C), 122.7, 122.4, 119.6, 63.1, 39.6, 32.7, 29.0, 26.0 (3C), 25.7, 24.4, 21.5, 18.4, -5.2 (2C) ppm; HRMS (ESI): calcd. for $\mathrm{C}_{26} \mathrm{H}_{40} \mathrm{NO}_{4} \mathrm{SSi}[\mathrm{M}+\mathrm{H}]^{+}: 490.2442$ found: 490.2437

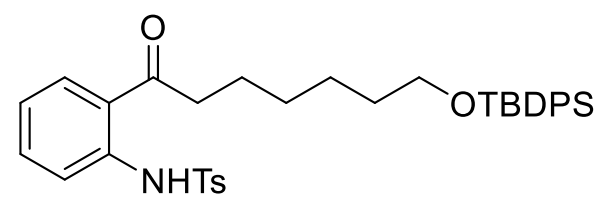

5al

N-(2-(7-((tert-butyldiphenylsilyl)oxy)heptanoyl)phenyl)-4-methylbenzenesulfonamide (5al) was isolated through column chromatography on silica gel (petroleum ether: $\mathrm{EtOAc}=10: 1)$ as a white solid $(75 \mathrm{mg}, 61 \%$ yield $) .{ }^{1} \mathrm{H}$ NMR $(400 \mathrm{MHz}$, $\left.\mathrm{CDCl}_{3}\right) \delta=11.46(\mathrm{~s}, 1 \mathrm{H}), 7.78(\mathrm{dd}, J=8.0,1.6 \mathrm{~Hz}, 1 \mathrm{H}), 7.75-7.63(\mathrm{~m}, 7 \mathrm{H}), 7.48-$ $7.41(\mathrm{~m}, 2 \mathrm{H}), 7.43-7.33(\mathrm{~m}, 5 \mathrm{H}), 7.19(\mathrm{~d}, J=8.0 \mathrm{~Hz}, 2 \mathrm{H}), 7.05(\mathrm{~s}, 1 \mathrm{H}), 3.67(\mathrm{t}, J$ $=6.4 \mathrm{~Hz}, 2 \mathrm{H}), 2.84(\mathrm{t}, J=7.4 \mathrm{~Hz}, 2 \mathrm{H}), 2.33(\mathrm{~s}, 3 \mathrm{H}), 1.64-1.53(\mathrm{~m}, 4 \mathrm{H}), 1.42-1.33$ (m, 2H), $1.35-1.22(\mathrm{~m}, 2 \mathrm{H}), 1.05(\mathrm{~s}, 9 \mathrm{H}) \mathrm{ppm} ;{ }^{13} \mathrm{C} \mathrm{NMR}\left(101 \mathrm{MHz}, \mathrm{CDCl}_{3}\right) \delta=204.6,143.7,139.9,136.6,135.5(4 \mathrm{C}), 134.6$, 134.0, 130.9, 129.6 (2C), 129.5 (2C), 127.6 (4C), 127.2 (2C), 122.7 (2C), 122.4, 119.6, 63.8, 39.6, 32.3, 28.9, 26.9 (3C), 25.6, 


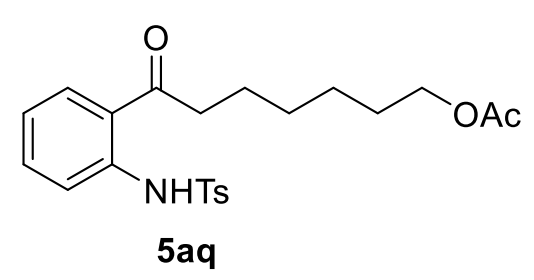

7-(2-((4-Methylphenyl)sulfonamido)phenyl)-7-oxoheptyl acetate (5aq) was isolated through column chromatography on silica gel (petroleum ether: $\mathrm{EtOAc}=10: 1)$ as a white solid (50 mg, $60 \%$ yield). ${ }^{1} \mathrm{H}$ NMR $\left(500 \mathrm{MHz}, \mathrm{CDCl}_{3}\right) \delta=11.45(\mathrm{~s}, 1 \mathrm{H}), 7.80$ (dd, $J=$ 8.1, $1.5 \mathrm{~Hz}, 1 \mathrm{H}), 7.75-7.66(\mathrm{~m}, 3 \mathrm{H}), 7.44(\mathrm{~d}, J=1.6 \mathrm{~Hz}, 1 \mathrm{H}), 7.22(\mathrm{~d}, J=7.9 \mathrm{~Hz}, 2 \mathrm{H})$, 7.08-7.02 (m, 1H), 4.07 (t, $J=6.7 \mathrm{~Hz}, 2 \mathrm{H}), 2.89$ (t, $J=7.3 \mathrm{~Hz}, 2 \mathrm{H}), 2.36(\mathrm{~s}, 3 \mathrm{H}), 2.05$ $(\mathrm{s}, 3 \mathrm{H}), 1.70-1.61(\mathrm{~m}, 4 \mathrm{H}), 1.43-1.29(\mathrm{~m}, 4 \mathrm{H}) \mathrm{ppm} ;{ }^{13} \mathrm{C} \mathrm{NMR}\left(126 \mathrm{MHz}, \mathrm{CDCl}_{3}\right) \delta=$ 204.4, 171.2, 143.8, 140.0, 136.7, 134.6, 131.0, 129.6 (2C), 127.3 (2C), 122.7, 122.3, 119.5, 64.4, 39.5, 28.8, 28.5, 25.8, 24.2, 21.5, 21.0 ppm; HRMS (ESI): calcd. for $\mathrm{C}_{22} \mathrm{H}_{27} \mathrm{NO}_{5} \mathrm{SNa}[\mathrm{M}+\mathrm{Na}]^{+}: 440.1502$ found: 440.1506

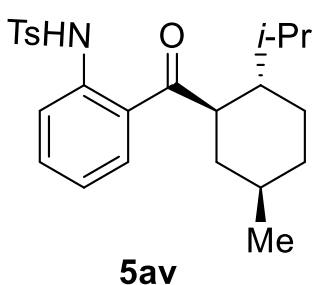

5 av

$N-(2-((1 R, 2 S, 5 R)-2-i s o p r o p y l-5-m e t h y l c y c l o h e x a n e-1-c a r b o n y l) p h e n y l)-4-$ methylbenzenesulfonamide (5av) was isolated through column chromatography on silica gel (petroleum ether: EtOAc $=10: 1)$ as a white solid (56 mg, $68 \%$ yield). ${ }^{1} \mathrm{H}$ NMR $\left(500 \mathrm{MHz}, \mathrm{CDCl}_{3}\right)$ $\delta=11.55(\mathrm{~s}, 1 \mathrm{H}), 7.86(\mathrm{dd}, J=8.1,1.5 \mathrm{~Hz}, 1 \mathrm{H}), 7.78(\mathrm{dd}, J=8.4,1.1 \mathrm{~Hz}, 1 \mathrm{H}), 7.72-7.65(\mathrm{~m}, 2 \mathrm{H})$, $7.47(\mathrm{~d}, J=1.5 \mathrm{~Hz}, 1 \mathrm{H}), 7.18(\mathrm{~d}, J=8.1 \mathrm{~Hz}, 2 \mathrm{H}), 7.10(\mathrm{~d}, J=7.1 \mathrm{~Hz}, 1 \mathrm{H}), 3.28(\mathrm{~d}, J=3.2 \mathrm{~Hz}, 1 \mathrm{H})$, $2.34(\mathrm{~s}, 3 \mathrm{H}), 1.82-1.71(\mathrm{~m}, 3 \mathrm{H}), 1.61-1.52(\mathrm{~m}, 1 \mathrm{H}), 1.44-1.34(\mathrm{~m}, 2 \mathrm{H}), 1.08(\mathrm{dd}, J=12.5,3.1 \mathrm{~Hz}$, $1 \mathrm{H}), 1.03-0.93(\mathrm{~m}, 1 \mathrm{H}), 0.91(\mathrm{~d}, J=12.6 \mathrm{~Hz}, 1 \mathrm{H}), 0.86(\mathrm{~d}, J=3.2 \mathrm{~Hz}, 3 \mathrm{H}), 0.85(\mathrm{~d}, J=3.5 \mathrm{~Hz}, 3 \mathrm{H}), 0.61(\mathrm{~d}, J=7.0 \mathrm{~Hz}, 3 \mathrm{H})$ ppm; ${ }^{13} \mathrm{C}$ NMR $\left(126 \mathrm{MHz}, \mathrm{CDCl}_{3}\right) \delta=208.8,143.6,140.4,136.8,134.6,130.4,129.6$ (2C), $127.2(2 \mathrm{C}), 123.0,122.8,120.3$, 48.9, 43.8, 40.2, 34.5, 32.6, 28.8, 24.0, 22.3, 21.6, 21.5, 16.5 ppm; HRMS (ESI): calcd. for $\mathrm{C}_{24} \mathrm{H}_{31} \mathrm{NO}_{3} \mathrm{SNa}[\mathrm{M}+\mathrm{Na}]^{+}: 436.1917$ found: 436.1926.

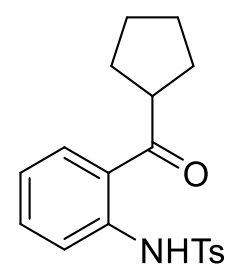

5aw

$N$-(2-(cyclopentanecarbonyl)phenyl)-4-methylbenzenesulfonamide (5aw) was isolated through column chromatography on silica gel (petroleum ether: EtOAc $=10: 1)$ as a white solid (35 mg, $51 \%$ yield). ${ }^{1} \mathrm{H} \mathrm{NMR}$ $\left(500 \mathrm{MHz}, \mathrm{CDCl}_{3}\right) \delta=11.41(\mathrm{~s}, 1 \mathrm{H}), 7.81(\mathrm{dd}, J=8.1,1.4 \mathrm{~Hz}, 1 \mathrm{H}), 7.73-7.65(\mathrm{~m}, 3 \mathrm{H}), 7.48-7.41(\mathrm{~m}, 1 \mathrm{H})$, $7.20(\mathrm{~d}, J=8.0 \mathrm{~Hz}, 2 \mathrm{H}), 7.11-7.04(\mathrm{~m}, 1 \mathrm{H}), 3.75-3.46(\mathrm{~m}, 1 \mathrm{H}), 2.35(\mathrm{~s}, 3 \mathrm{H}), 1.90-1.76(\mathrm{~m}, 2 \mathrm{H}), 1.75-1.53$ $(\mathrm{m}, 6 \mathrm{H}) \mathrm{ppm} ;{ }^{13} \mathrm{C} \mathrm{NMR}\left(126 \mathrm{MHz}, \mathrm{CDCl}_{3}\right) \delta=207.0,143.7,140.2,136.7,134.3,131.2,129.6(2 \mathrm{C}), 127.2$ (2C), 122.9, 122.7, 120.2, 47.1, 30.1 (2C), 26.3 (2C), 21.5 ppm; HRMS (ESI): calcd. for $\mathrm{C}_{19} \mathrm{H}_{21} \mathrm{NO}_{3} \mathrm{SNa}$ $[\mathrm{M}+\mathrm{Na}]^{+}: 366.1134$ found: 366.1147 .

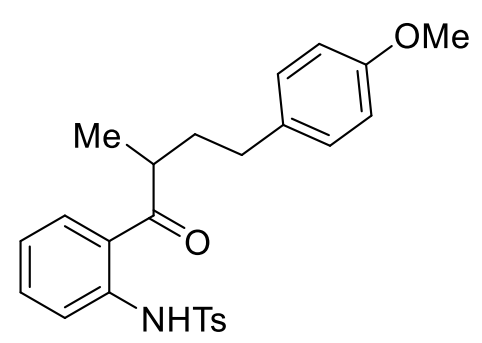

5 ax

$\mathrm{N}$-(2-(4-(4-methoxyphenyl)-2-methylbutanoyl)phenyl)-4-methylbenzenesulfonamide (5ax) was isolated through column chromatography on silica gel (petroleum ether: EtOAc=10:1) as a white solid (54 mg, $62 \%$ yield). ${ }^{1} \mathrm{H}$ NMR $\left(500 \mathrm{MHz}, \mathrm{CDCl}_{3}\right) \delta=11.45(\mathrm{~s}, 1 \mathrm{H}), 7.74$ (d, $J=8.4 \mathrm{~Hz}, 1 \mathrm{H}), 7.70$ (d, $J=8.0 \mathrm{~Hz}, 2 \mathrm{H}), 7.60$ (d, $J=8.0 \mathrm{~Hz}, 1 \mathrm{H}), 7.45$ (t, $J=7.9 \mathrm{~Hz}$, $1 \mathrm{H}), 7.16(\mathrm{~d}, J=7.9 \mathrm{~Hz}, 2 \mathrm{H}), 7.03-6.94(\mathrm{~m}, 3 \mathrm{H}), 6.80(\mathrm{~d}, J=8.1 \mathrm{~Hz}, 2 \mathrm{H}), 3.78(\mathrm{~s}, 3 \mathrm{H})$, 3.41-3.32 (m, 1H), 2.50-2.40 (m, 2H), $2.27(\mathrm{~s}, 3 \mathrm{H}), 2.04-1.92(\mathrm{~m}, 1 \mathrm{H}), 1.64-1.50(\mathrm{~m}, 1 \mathrm{H})$, $1.09(\mathrm{~d}, J=6.8 \mathrm{~Hz}, 3 \mathrm{H}) \mathrm{ppm} ;{ }^{13} \mathrm{C}$ NMR $\left(126 \mathrm{MHz}, \mathrm{CDCl}_{3}\right) \delta=208.4,158.0,143.8,140.4$, 136.7, 134.6, 133.4, 130.7, 129.6 (2C), 129.4 (2C), 127.2 (2C), 122.8, 122.1, 120.2, 113.8 (2C), 55.3, 40.3, 35.5, 32.4, 21.5, 17.4 ppm; HRMS (ESI): calcd. for $\mathrm{C}_{25} \mathrm{H}_{27} \mathrm{NO}_{4} \mathrm{SNa}[\mathrm{M}+\mathrm{Na}]^{+}: 460.1553$ found: 460.1559.<smiles>COc1ccc(N)c(C(=O)CCCCCC[O+]S)c1</smiles>

5bk
N-(2-(7-((tert-butyldimethylsilyl)oxy)heptanoyl)-4-methoxyphenyl)-4-methylbenzenesulfonamide (5bk) was isolated through column chromatography on silica gel (petroleum ether: $\mathrm{EtOAc}=5: 1)$ as a white solid $(76 \mathrm{mg}, 73 \%$ yield). ${ }^{1} \mathrm{H}$ NMR $\left(400 \mathrm{MHz}, \mathrm{CDCl}_{3}\right) \delta=10.59(\mathrm{~s}, 1 \mathrm{H}), 7.68(\mathrm{~d}, J=9.0 \mathrm{~Hz}, 1 \mathrm{H}), 7.57$ (d, $J=8.4 \mathrm{~Hz}, 2 \mathrm{H}), 7.21(\mathrm{~d}, J=2.9 \mathrm{~Hz}, 1 \mathrm{H}), 7.20-7.13(\mathrm{~m}, 2 \mathrm{H}), 7.04$ (dd, $J=$ 9.1, $2.9 \mathrm{~Hz}, 1 \mathrm{H}), 3.80$ (s, 3H), $3.61(\mathrm{t}, J=6.5 \mathrm{~Hz}, 2 \mathrm{H}), 2.71(\mathrm{t}, J=7.4 \mathrm{~Hz}, 2 \mathrm{H})$, 
$2.34(\mathrm{~s}, 3 \mathrm{H}), 1.57-1.47(\mathrm{~m}, 4 \mathrm{H}), 1.38-1.26(\mathrm{~m}, 4 \mathrm{H}), 0.90(\mathrm{~s}, 9 \mathrm{H}), 0.06(\mathrm{~s}, 6 \mathrm{H}) \mathrm{ppm} ;{ }^{13} \mathrm{C} \mathrm{NMR}\left(101 \mathrm{MHz}, \mathrm{CDCl}_{3}\right) \delta=204.1$, 155.4, 143.5, 136.3, 132.2, 129.4 (2C), 127.2 (2C), 125.3, 123.5, 119.4, 115.8, 63.1, 55.7, 39.6, 32.7, 29.0, 26.0 (3C), 25.7, 24.2, 21.5, 18.4, -5.3 (2C); HRMS (ESI): calcd. for $\mathrm{C}_{27} \mathrm{H}_{41} \mathrm{NO}_{5} \mathrm{SSiNa}[\mathrm{M}+\mathrm{Na}]^{+}: 542.2367$ found: 542.2368 .

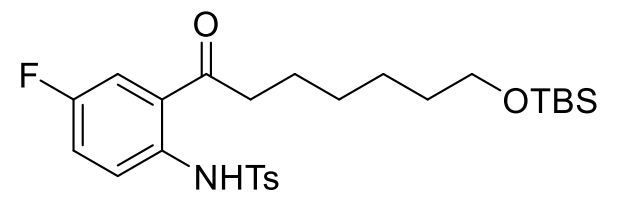

$5 \mathrm{ck}$

$N-(2-(7-(($ tert-Butyldimethylsilyl)oxy)heptanoyl)-4-fluorophenyl)-4-methylbenzenesulfonamide (5ck) was isolated through column chromatography on silica gel (petroleum ether: $\mathrm{EtOAc}=10: 1)$ as a white solid (64 mg, $63 \%$ yield). ${ }^{1} \mathrm{H}$ NMR $\left(400 \mathrm{MHz}, \mathrm{CDCl}_{3}\right) \delta=10.96(\mathrm{~s}, 1 \mathrm{H}), 7.73(\mathrm{dd}, J=9.2,4.9 \mathrm{~Hz}, 1 \mathrm{H}), 7.67-7.60(\mathrm{~m}$, 2H), $7.43(\mathrm{dd}, J=9.1,3.0 \mathrm{~Hz}, 1 \mathrm{H}), 7.24-7.16(\mathrm{~m}, 3 \mathrm{H}), 3.61$ (t, $J=6.5 \mathrm{~Hz}, 2 \mathrm{H})$, $2.77(\mathrm{t}, J=7.4 \mathrm{~Hz}, 2 \mathrm{H}), 2.36(\mathrm{~s}, 3 \mathrm{H}), 1.62-1.48(\mathrm{~m}, 4 \mathrm{H}), 1.39-1.28(\mathrm{~m}, 4 \mathrm{H}), 0.90$ (s, 9H), $0.06(\mathrm{~s}, 6 \mathrm{H}) \mathrm{ppm} ;{ }^{13} \mathrm{C}$ NMR $\left(101 \mathrm{MHz}, \mathrm{CDCl}_{3}\right) \delta=203.39\left(\mathrm{C}-\mathrm{F},{ }^{4} J_{\mathrm{C}-\mathrm{F}}=2.2 \mathrm{~Hz}\right), 203.37\left(\mathrm{C}-\mathrm{F},{ }^{4} J_{\mathrm{C}-\mathrm{F}}=2.2 \mathrm{~Hz}\right), 159.1$ $\left(\mathrm{C}-\mathrm{F},{ }^{1} J_{\mathrm{C}-\mathrm{F}}=244.7 \mathrm{~Hz}\right), 156.7\left(\mathrm{C}-\mathrm{F},{ }^{1} J_{\mathrm{C}-\mathrm{F}}=244.7 \mathrm{~Hz}\right), 143.9,136.2,135.8\left(\mathrm{C}-\mathrm{F},{ }^{4} J_{\mathrm{C}-\mathrm{F}}=2.6 \mathrm{~Hz}\right), 135.7\left(\mathrm{C}-\mathrm{F},{ }^{4} J_{\mathrm{C}-\mathrm{F}}=2.6 \mathrm{~Hz}\right)$, $129.6(2 \mathrm{C}), 127.2(2 \mathrm{C}), 124.3\left(\mathrm{C}-\mathrm{F},{ }^{3} J_{\mathrm{C}-\mathrm{F}}=5.8 \mathrm{~Hz}\right), 124.2\left(\mathrm{C}-\mathrm{F},{ }^{3} J_{\mathrm{C}-\mathrm{F}}=5.8 \mathrm{~Hz}\right), 122.7\left(\mathrm{C}-\mathrm{F},{ }^{3} J_{\mathrm{C}-\mathrm{F}}=7.4 \mathrm{~Hz}\right), 122.6\left(\mathrm{C}-\mathrm{F},{ }^{3} J_{\mathrm{C}-}\right.$ $\mathrm{F}=7.4 \mathrm{~Hz}), 121.7\left(\mathrm{C}-\mathrm{F},{ }^{2} J_{\mathrm{C}-\mathrm{F}}=22.4 \mathrm{~Hz}\right), 121.5\left(\mathrm{C}-\mathrm{F},{ }^{2} J_{\mathrm{C}-\mathrm{F}}=22.4 \mathrm{~Hz}\right), 116.9\left(\mathrm{C}-\mathrm{F},{ }^{2} J_{\mathrm{C}-\mathrm{F}}=23.4 \mathrm{~Hz}\right), 116.7\left(\mathrm{C}-\mathrm{F},{ }^{2} J_{\mathrm{C}-\mathrm{F}}=23.4\right.$ $\mathrm{Hz}), 63.0,39.7,32.6,28.9,26.0$ (3C), 25.6, 24.1, 21.5, 18.4, -5.3 (2C) ppm; ${ }^{19} \mathrm{~F}$ NMR $\left(376 \mathrm{MHz}, \mathrm{CDCl}_{3}\right) \delta=-111.55--$ 121.87 (m, 1F)ppm; HRMS (ESI): calcd. for $\mathrm{C}_{26} \mathrm{H}_{39} \mathrm{FNO}_{4} \mathrm{SSi}[\mathrm{M}+\mathrm{H}]^{+}: 508.2353$ found: 508.2353.<smiles>[NH3+]c1cc(C(F)(F)F)ccc1C(=O)CCCCCC[OH+]</smiles>

$5 d k$

N-(2-(7-((tert-Butyldimethylsilyl)oxy)heptanoyl)-5-(trifluoromethyl)phenyl)-4methylbenzenesulfonamide (5dk) was isolated through column chromatography on silica gel (petroleum ether: EtOAc $=10: 1)$ as a white solid (39 $\mathrm{mg}, 35 \%$ yield). ${ }^{1} \mathrm{H}$ NMR $\left(400 \mathrm{MHz}, \mathrm{CDCl}_{3}\right) \delta=11.44(\mathrm{~s}, 1 \mathrm{H}), 8.05-7.96(\mathrm{~m}, 1 \mathrm{H}), 7.91$ (d, $J=8.3 \mathrm{~Hz}, 1 \mathrm{H}), 7.80-7.69(\mathrm{~m}, 2 \mathrm{H}), 7.31-7.21(\mathrm{~m}, 3 \mathrm{H}), 3.61(\mathrm{t}, J=6.4 \mathrm{~Hz}$, 2H), 2.91 (t, $J=7.4 \mathrm{~Hz}, 2 \mathrm{H}), 2.37$ (s, 3H), 1.71-1.60 (m, 2H), 1.57-1.47 (m, 2H), 1.39-1.32 (m, 4H), $0.90(\mathrm{~s}, 9 \mathrm{H}), 0.05$ (s, 6H) ppm; ${ }^{13} \mathrm{C} \mathrm{NMR}\left(101 \mathrm{MHz}, \mathrm{CDCl}_{3}\right) \delta=204.0,144.3,140.4,136.1,135.8(\mathrm{C}-\mathrm{F}$, $\left.{ }^{2} J_{\mathrm{C}-\mathrm{F}}=33.2 \mathrm{~Hz}\right), 135.5\left(\mathrm{C}-\mathrm{F},{ }^{2} J_{\mathrm{C}-\mathrm{F}}=33.2 \mathrm{~Hz}\right), 131.5,129.8(2 \mathrm{C}), 127.3(2 \mathrm{C}), 124.3\left(\mathrm{C}-\mathrm{F},{ }^{1} J_{\mathrm{C}-\mathrm{F}}=274.7 \mathrm{~Hz}\right), 124.1,121.6$ $\left(\mathrm{C}-\mathrm{F},{ }^{1} J_{\mathrm{C}-\mathrm{F}}=274.7 \mathrm{~Hz}\right), 118.90\left(\mathrm{C}-\mathrm{F},{ }^{3} J_{\mathrm{C}-\mathrm{F}}=3.6 \mathrm{~Hz}\right), 118.87\left(\mathrm{C}-\mathrm{F},{ }^{3} J_{\mathrm{C}-\mathrm{F}}=3.6 \mathrm{~Hz}\right), 116.3\left(\mathrm{C}-\mathrm{F},{ }^{3} J_{\mathrm{C}-\mathrm{F}}=3.9 \mathrm{~Hz}\right), 116.2(\mathrm{C}-\mathrm{F}$, $\left.{ }^{3} J_{\mathrm{C}-\mathrm{F}}=3.9 \mathrm{~Hz}\right), 63.1,39.9,32.6,28.9,26.0(3 \mathrm{C}), 25.6,24.2,21.6,18.4,5.3(2 \mathrm{C}) \mathrm{ppm} ;{ }^{19} \mathrm{~F}$ NMR $\left(376 \mathrm{MHz}, \mathrm{CDCl}_{3}\right) \delta=-$ 63.76 (s, 3F)ppm; HRMS (ESI): calcd. for $\mathrm{C}_{27} \mathrm{H}_{39} \mathrm{~F}_{3} \mathrm{NO}_{4} \mathrm{SSi}[\mathrm{M}+\mathrm{H}]^{+}: 558.2321$ found: 558.2322 .

\section{Derivatizations of the Products}

\section{Synthesis of 2-Nonanoylphenyl Trifluoromethanesulfonate (6)}

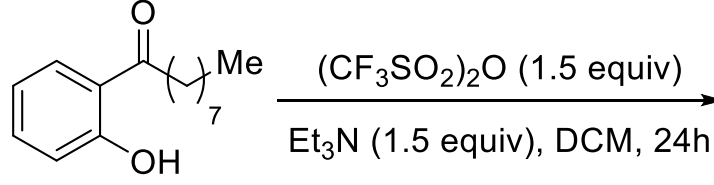

$3 a b$<smiles>CC(C)C(=O)c1ccccc1[O-]</smiles>

$6,92 \%$

The phenol 3ab (93.7 mg, $0.40 \mathrm{mmol}, 1$ equiv) was dissolved in DCM $(0.8 \mathrm{~mL})$, to which $\mathrm{Et}_{3} \mathrm{~N}(60.7 \mathrm{mg}, 0.60 \mathrm{mmol}, 1.5$ equiv) was added dropwise. After cooling to $0{ }^{\circ} \mathrm{C}$, trifluoromethanesulfonic anhydride ( $169.3 \mathrm{mg}, 0.60 \mathrm{mmol}, 1.5$ equiv) was added dropwise to the mixture. After stirring at room temperature for $24 \mathrm{~h}$, the reaction was quenched with water. The organic layer was separated and the aqueous phase was extracted with EtOAc. The combined organic phases were washed with water and saturated aqueous $\mathrm{NaCl}$ solution, dried over $\mathrm{Na}_{2} \mathrm{SO}_{4}$, filtered and concentrated under reduced pressure. The residue was purified through column chromatography on silica gel with the mixture eluent of petroleum ether and ethyl acetate (50:1) to 
give the title compound 6 as a yellow oil (134.8 mg, $92 \%$ yield). ${ }^{1} \mathrm{H}$ NMR $\left(400 \mathrm{MHz}, \mathrm{CDCl}_{3}\right) \delta=7.76(\mathrm{dd}, J=7.7,1.8 \mathrm{~Hz}$, $1 \mathrm{H}), 7.62-7.55(\mathrm{~m}, 1 \mathrm{H}), 7.51-7.44(\mathrm{~m}, 1 \mathrm{H}), 7.34(\mathrm{dd}, J=8.3,1.1 \mathrm{~Hz}, 1 \mathrm{H}), 2.93(\mathrm{t}, J=7.3 \mathrm{~Hz}, 2 \mathrm{H}), 1.78-1.66(\mathrm{~m}, 2 \mathrm{H}), 1.39-$ $1.23(\mathrm{~m}, 10 \mathrm{H}), 0.87(\mathrm{t}, J=7.0 \mathrm{~Hz}, 3 \mathrm{H}) \mathrm{ppm} ;{ }^{13} \mathrm{C} \mathrm{NMR}\left(101 \mathrm{MHz}, \mathrm{CDCl}_{3}\right) \delta=199.6,146.6,133.2,132.6,130.2,128.5,122.7$, 118.6 (q, $J=320.5 \mathrm{~Hz}$ ), 41.8, 31.8, 29.3, 29.1 (2C), 23.9, 22.6, 14.1 ppm; HRMS (ESI): calcd. for $\mathrm{C}_{16} \mathrm{H}_{21} \mathrm{~F}_{3} \mathrm{O}_{4} \mathrm{SNa}$ [M+Na] 389.1005 found: 389.1011 .

\section{Synthesis of 1-([1,1'-Biphenyl]-2-yl)nonan-1-one (7)}

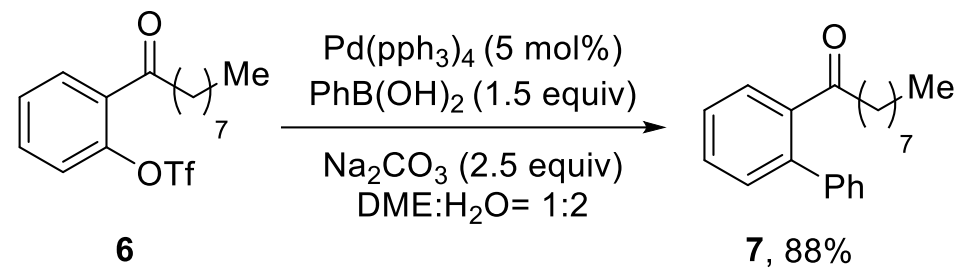

Under nitrogen, the triflate $6(73.3 \mathrm{mg}, 0.20 \mathrm{mmol}, 1.0$ equiv) and phenylboronic acid ( $36.6 \mathrm{mg}, 0.30 \mathrm{mmol}, 1.5 \mathrm{equiv})$ was dissolved in anhydrous 1,2-dimethoxyethane $(0.25 \mathrm{~mL})$ and water $(0.50 \mathrm{~mL})$, After addition of $\mathrm{Na}_{2} \mathrm{CO}_{3}(53.0 \mathrm{mg}, 0.50 \mathrm{mmol}$, 2.5 equiv) and $\mathrm{Pd}\left(\mathrm{PPh}_{3}\right)_{4}\left(11.6 \mathrm{mg}, 0.01 \mathrm{mmol}, 5 \mathrm{~mol} \%\right.$ ), the mixture was stirred at $100{ }^{\circ} \mathrm{C}$ (oil bath) for 12 hours. After cooling, the reaction mixture was passed through a plug of silica gel (washed with DCM) and the solvent was emoved under reduced pressure. Purification by column chromatography (petroleum ether) afforded the title compound 7 as a yellow oil (52 $\mathrm{mg}, 88 \%$ yield). ${ }^{1} \mathrm{H}$ NMR $\left(400 \mathrm{MHz}, \mathrm{CDCl}_{3}\right) \delta=7.44-7.36(\mathrm{~m}, 2 \mathrm{H}), 7.35-7.28(\mathrm{~m}, 5 \mathrm{H}), 7.27-7.17(\mathrm{~m}, 2 \mathrm{H}), 2.14(\mathrm{t}, J=7.5$ $\mathrm{Hz}, 2 \mathrm{H}), 1.38-1.24(\mathrm{~m}, 2 \mathrm{H}), 1.20-1.13(\mathrm{~m}, 2 \mathrm{H}), 1.11-1.04(\mathrm{~m}, 4 \mathrm{H}), 1.02-0.88(\mathrm{~m}, 4 \mathrm{H}), 0.78(\mathrm{t}, J=7.1 \mathrm{~Hz}, 3 \mathrm{H}) \mathrm{ppm} ;{ }^{13} \mathrm{C} \mathrm{NMR}$ $\left(126 \mathrm{MHz}, \mathrm{CDCl}_{3}\right) \delta=208.4,141.3,140.7,140.0,130.3,130.1,128.9$ (2C), 128.7 (2C), 127.8, 127.6, 127.5, 43.0, 31.8, 29.1, 29.04, 28.96, 24.5, 22.6, 14.1 ppm; HRMS (ESI): calcd. for $\mathrm{C}_{21} \mathrm{H}_{26} \mathrm{ONa}[\mathrm{M}+\mathrm{Na}]^{+}: 317.1876$ found: 317.1876 .

\section{Synthesis of 2-(6-Fluorohexanoyl)phenyl Trifluoromethanesulfonate (9)}<smiles>CC(F)(F)C(=O)c1ccccc1O</smiles>

3ah

$$
\underset{\mathrm{Et}_{3} \mathrm{~N}(2.0 \text { equiv }), \mathrm{DCM}, 12 \mathrm{~h}}{\stackrel{\left(\mathrm{CF}_{3} \mathrm{SO}_{2}\right)_{2} \mathrm{O}(2.0 \text { equiv })}{\longrightarrow}}
$$<smiles>O=C(c1ccccc1Br)C(F)(F)F</smiles>

$9,75 \%$

The phenol 3ah (90.4 mg, $0.43 \mathrm{mmol}, 1$ equiv) was dissolved in DCM $(0.8 \mathrm{~mL})$, to which $\mathrm{Et}_{3} \mathrm{~N}(87.0 \mathrm{mg}, 0.86 \mathrm{mmol}, 2.0$ equiv) was added dropwise. After cooling to $0{ }^{\circ} \mathrm{C}$, trifluoromethanesulfonic anhydride ( $242.6 \mathrm{mg}, 0.86 \mathrm{mmol}, 2.0$ equiv) was added dropwise. After stirring at room temperature for $12 \mathrm{~h}$, the reaction was quenched with water. The organic layer was separated and the aqueous phase was extracted with EtOAc. The combined organic phases were washed with water and saturated aqueous $\mathrm{NaCl}$ solution, dried over $\mathrm{Na}_{2} \mathrm{SO}_{4}$, filtered and concentrated under reduced pressure. The residue was purified through column chromatography on silica gel with the mixture eluent of petroleum ether and ethyl acetate (50:1) to give the title compound 9 as a yellow oil $\left(110.4 \mathrm{mg}, 75 \%\right.$ yield). ${ }^{1} \mathrm{H}$ NMR $\left(500 \mathrm{MHz}, \mathrm{CDCl}_{3}\right) \delta=7.77(\mathrm{dd}, J=7.7,1.8 \mathrm{~Hz}$, 1H), 7.64-7.56 (m, 1H), 7.51-7.43 (m, 1H), 7.34 (d, $J=8.2 \mathrm{~Hz}, 1 \mathrm{H}), 4.45$ (dt, $J=47.3,6.1 \mathrm{~Hz}, 2 \mathrm{H}), 2.97$ (t, $J=7.3 \mathrm{~Hz}, 2 \mathrm{H})$, $1.84-1.66(\mathrm{~m}, 4 \mathrm{H}), 1.58-1.41$ (m, 2H) ppm; ${ }^{13} \mathrm{C} \mathrm{NMR}\left(126 \mathrm{MHz}, \mathrm{CDCl}_{3}\right) \delta=199.2,146.6,133.4,132.4,130.2,128.6,122.7$, $122.4\left(\mathrm{C}-\mathrm{F},{ }^{1} J_{\mathrm{C}-\mathrm{F}}=321.3 \mathrm{~Hz}\right), 119.9\left(\mathrm{C}-\mathrm{F},{ }^{1} J_{\mathrm{C}-\mathrm{F}}=321.3 \mathrm{~Hz}\right), 117.3\left(\mathrm{C}-\mathrm{F},{ }^{1} J_{\mathrm{C}-\mathrm{F}}=321.3 \mathrm{~Hz}\right), 114.8\left(\mathrm{C}-\mathrm{F},{ }^{1} J_{\mathrm{C}-\mathrm{F}}=321.3 \mathrm{~Hz}\right)$, $84.5\left(\mathrm{C}-\mathrm{F},{ }^{1} J_{\mathrm{C}-\mathrm{F}}=164.4 \mathrm{~Hz}\right), 83.2\left(\mathrm{C}-\mathrm{F},{ }^{1} J_{\mathrm{C}-\mathrm{F}}=164.4 \mathrm{~Hz}\right), 41.5,30.3\left(\mathrm{C}-\mathrm{F},{ }^{2} J_{\mathrm{C}-\mathrm{F}}=19.4 \mathrm{~Hz}\right), 30.1\left(\mathrm{C}-\mathrm{F},{ }^{2} J_{\mathrm{C}-\mathrm{F}}=19.4 \mathrm{~Hz}\right)$, $24.8\left(\mathrm{C}-\mathrm{F},{ }^{3} J_{\mathrm{C}-\mathrm{F}}=5.4 \mathrm{~Hz}\right), 24.7\left(\mathrm{C}-\mathrm{F},{ }^{3} J_{\mathrm{C}-\mathrm{F}}=5.4 \mathrm{~Hz}\right), 23.4 \mathrm{ppm} ;{ }^{19} \mathrm{~F}$ NMR $\left(471 \mathrm{MHz}, \mathrm{CDCl}_{3}\right) \delta=-73.40(\mathrm{~s}, 3 \mathrm{~F}),-218.53(\mathrm{~s}$, 1F) ppm; HRMS (ESI): calcd. for $\mathrm{C}_{13} \mathrm{H}_{14} \mathrm{~F}_{4} \mathrm{O}_{4} \mathrm{SNa}[\mathrm{M}+\mathrm{Na}]^{+}: 365.0441$ found:365.0453. 


\section{Synthesis of (Z)-3-(5-Fluoropentylidene)isobenzofuran-1(3H)-one (10) ${ }^{15}$}

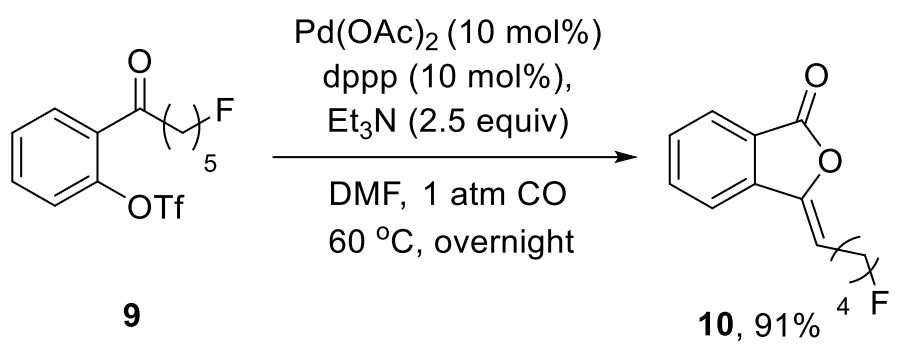

A mixture of the triflate 9 (102.7 mg, $0.3 \mathrm{mmol}, 1.0$ equiv), $\mathrm{Et}_{3} \mathrm{~N}$ (60.7 mg, $0.75 \mathrm{mmol}, 2.5$ equiv), $\mathrm{Pd}(\mathrm{OAc})_{2}(6.7 \mathrm{mg}, 0.03$ $\mathrm{mmol}, 10 \mathrm{~mol} \%$ ) and 1,3-bis(diphenylphosphino)propane (12.4 mg, $0.03 \mathrm{mmol}, 10 \mathrm{~mol} \%)$ in DMF (3.0 mL) was degassed by two freeze/pump/thaw cycles. The reaction vessel was filled with $\mathrm{CO}$ and stirred at $60{ }^{\circ} \mathrm{C}$ (oil bath) under a balloon of $\mathrm{CO}$ for $12 \mathrm{~h}$. The reaction mixture was diluted with brine and extracted with $\mathrm{Et}_{2} \mathrm{O}$. The combined organic phases were washed with brine, dried over $\mathrm{MgSO}_{4}$, filtered and evaporated under reduced pressure. Purification by flash column chromatography on silica gel (petroleum ether /ethyl acetate $=20: 1)$ afforded the title compound $\mathbf{1 0}$ as a colorless oil $(60.1 \mathrm{mg}, 91 \%$ yield). ${ }^{1} \mathrm{H}$ NMR $\left(400 \mathrm{MHz}, \mathrm{CDCl}_{3}\right) \delta=7.89(\mathrm{dd}, J=7.7,0.9 \mathrm{~Hz}, 1 \mathrm{H}), 7.72-7.62(\mathrm{~m}, 2 \mathrm{H}), 7.55-7.49(\mathrm{~m}, 1 \mathrm{H}), 5.64(\mathrm{t}, J=7.8 \mathrm{~Hz}, 1 \mathrm{H})$, $4.49(\mathrm{dt}, J=47.2,5.9 \mathrm{~Hz}, 2 \mathrm{H}), 2.53(\mathrm{q}, J=7.5 \mathrm{~Hz}, 2 \mathrm{H}), 1.86-1.78(\mathrm{~m}, 2 \mathrm{H}), 1.78-1.59(\mathrm{~m}, 2 \mathrm{H}) \mathrm{ppm} ;{ }^{13} \mathrm{C} \mathrm{NMR}(101 \mathrm{MHz}$, $\left.\mathrm{CDCl}_{3}\right) \delta=167.1,146.0,139.4,134.3,129.5,125.3,124.4,119.7,108.7,84.6\left(\mathrm{C}-\mathrm{F},{ }^{1} J_{\mathrm{C}-\mathrm{F}}=164.5 \mathrm{~Hz}\right), 82.3\left(\mathrm{C}-\mathrm{F},{ }^{1} J_{\mathrm{C}-\mathrm{F}}=\right.$ $164.5 \mathrm{~Hz}), 30.1\left(\mathrm{C}-\mathrm{F},{ }^{2} J_{\mathrm{C}-\mathrm{F}}=19.7 \mathrm{~Hz}\right), 30.0\left(\mathrm{C}-\mathrm{F},{ }^{2} J_{\mathrm{C}-\mathrm{F}}=19.7 \mathrm{~Hz}\right), 25.3,25.0\left(\mathrm{C}-\mathrm{F},{ }^{3} J_{\mathrm{C}-\mathrm{F}}=4.9 \mathrm{~Hz}\right), 24.9\left(\mathrm{C}-\mathrm{F},{ }^{3} J_{\mathrm{C}-\mathrm{F}}=4.9\right.$ $\mathrm{Hz}) \mathrm{ppm} ;{ }^{19} \mathrm{~F}$ NMR $\left(471 \mathrm{MHz}, \mathrm{CDCl}_{3}\right) \delta=-218.31--219.04$ (m, 1F); HRMS (ESI): calcd. for $\mathrm{C}_{13} \mathrm{H}_{13} \mathrm{FO}_{2} \mathrm{Na}[\mathrm{M}+\mathrm{Na}]^{+}$: 243.0792 found: 243.0798 .

\section{Synthesis of Phenyl 4-Methoxy-3-nonanoylbenzoate (14)}<smiles>CC(C)CCCCCCC(=O)c1cc(C(=O)Oc2ccccc2)ccc1O</smiles>

3ib<smiles>COc1ccc(C(=O)Oc2ccccc2)cc1C(=O)C(C)C</smiles>

$14,78 \%$

The phenol 3ib (101.6 mg, $0.3 \mathrm{mmol}, 1$ equiv), $\mathrm{K}_{2} \mathrm{CO}_{3}(165.9 \mathrm{mg}, 1.2 \mathrm{mmol}, 4$ equiv) was dissolved in DMF (0.6 mL), to which $\mathrm{CH}_{3} \mathrm{I}$ (127.7 mg, $0.9 \mathrm{mmol}, 3.0$ equiv) was added. After stirring at $55^{\circ} \mathrm{C}$ (oil bath) for $8 \mathrm{~h}$, the reaction was quenched with water. The mixture was extracted with EtOAc and the combined organic phases were washed with water, brine, dried over $\mathrm{Na}_{2} \mathrm{SO}_{4}$, filtered and concentrated under reduced pressure. The residue was purified through column chromatography on silica gel with the mixture eluent of petroleum ether and ethyl acetate (10:1) to give the title compound $\mathbf{1 4}$ as a colorless oil (86 mg, $78 \%$ yield). ${ }^{1} \mathrm{H}$ NMR $\left(500 \mathrm{MHz}, \mathrm{CDCl}_{3}\right) \delta=8.46(\mathrm{~d}, J=2.3 \mathrm{~Hz}, 1 \mathrm{H}), 8.27$ (dd, $\left.J=8.7,2.3 \mathrm{~Hz}, 1 \mathrm{H}\right), 7.42(\mathrm{t}, J=7.9$ $\mathrm{Hz}, 2 \mathrm{H}), 7.27(\mathrm{~d}, J=8.2 \mathrm{~Hz}, 1 \mathrm{H}), 7.20(\mathrm{~d}, J=7.5 \mathrm{~Hz}, 2 \mathrm{H}), 7.06(\mathrm{~d}, J=8.7 \mathrm{~Hz}, 1 \mathrm{H}), 3.99(\mathrm{~s}, 3 \mathrm{H}), 2.97(\mathrm{t}, J=7.4 \mathrm{~Hz}, 2 \mathrm{H})$, $1.76-1.65(\mathrm{~m}, 2 \mathrm{H}), 1.37-1.25(\mathrm{~m}, 10 \mathrm{H}), 0.88(\mathrm{t}, J=6.7 \mathrm{~Hz}, 3 \mathrm{H}) \mathrm{ppm} ;{ }^{13} \mathrm{C} \mathrm{NMR}\left(126 \mathrm{MHz}, \mathrm{CDCl}_{3}\right) \delta=202.4,164.4,162.0$, 150.9, 135.2, 132.5, 129.5 (2C), 129.1, 125.9, 122.1, 121.7 (2C), 111.5, 56.0, 43.7, 31.9, 29.5 29.4, 29.2 , 24.3, $22.7,14.1$ ppm; HRMS (ESI): calcd. for $\mathrm{C}_{23} \mathrm{H}_{29} \mathrm{O}_{4}[\mathrm{M}+\mathrm{H}]^{+}: 369.2060$ found:369.2072. 
<smiles>CNC(=O)c1cc(C(=O)Oc2ccccc2)ccc1OC</smiles>

$\mathrm{PhNH}_{2}$

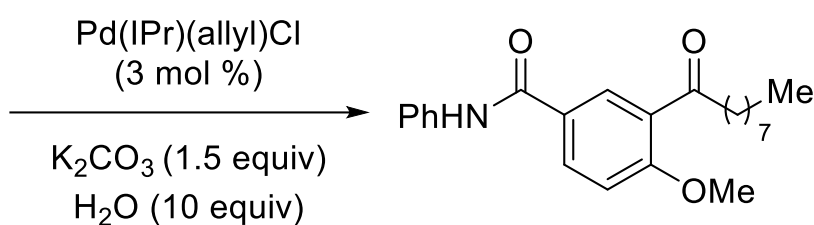

$11,87 \%$

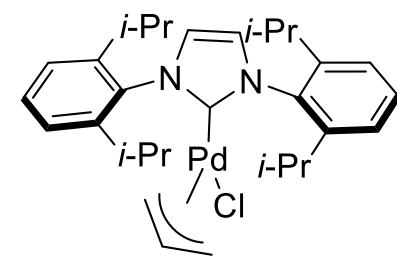

$\operatorname{Pd}(\operatorname{IPr})($ allyl $)(\mathrm{Cl})$

An oven dried screw-capped vial was charged with a magnetic stir bar, powdered $\mathrm{K}_{2} \mathrm{CO}_{3}(41.5 \mathrm{mg}, 0.3 \mathrm{mmol} 1.5$ equiv), $\operatorname{Pd}(\operatorname{IPr})($ allyl)Cl (3.44 mg, $0.06 \mathrm{mmol}, 3 \mathrm{~mol} \%$ ), the phenolic ester 14 (74.0 mg, $0.2 \mathrm{mmol}, 1$ equiv) and aniline (23.0 mg, 0.24 mmol, 1.2 equiv). The vial and contents were placed under vacuum and back-filled with $\mathrm{N}_{2}$ under a Schleck line three times. Dry toluene ( $1 \mathrm{~mL}, 0.2 \mathrm{M})$ and degassed water $(36.0 \mathrm{mg}, 2.0 \mathrm{mmol}, 10$ equiv) were then added successively under nitrogen. The vial was sealed and stirred vigorously at $110^{\circ} \mathrm{C}$ (oil bath) for $16 \mathrm{~h}$. After cooling to room temperature, the reaction mixture was diluted with ethyl acetate and filtered through a plug of silica gel (10 $\mathrm{mL}$ of EtOAc eluent). The crude mixture was concentrated in vacuo and was purified through column chromatography on silica gel (petroleum ether: EtOAc= 5:1) to give the title compound 11 as a white solid ( $87 \mathrm{mg}, 87 \%$ yield). ${ }^{1} \mathrm{H}$ NMR $\left(400 \mathrm{MHz}, \mathrm{CDCl}_{3}\right) \delta=8.20(\mathrm{~s}, 1 \mathrm{H}), 8.16$ $8.06(\mathrm{~m}, 2 \mathrm{H}), 7.65(\mathrm{~d}, J=8.0 \mathrm{~Hz}, 2 \mathrm{H}), 7.35(\mathrm{t}, J=7.8 \mathrm{~Hz}, 2 \mathrm{H}), 7.13(\mathrm{t}, J=7.4 \mathrm{~Hz}, 1 \mathrm{H}), 7.03(\mathrm{~d}, J=8.6 \mathrm{~Hz}, 1 \mathrm{H}), 3.95(\mathrm{~s}, 3 \mathrm{H})$, $2.96(\mathrm{t}, J=7.4 \mathrm{~Hz}, 2 \mathrm{H}), 1.74-1.55(\mathrm{~m}, 2 \mathrm{H}), 1.35-1.16(\mathrm{~m}, 10 \mathrm{H}), 0.88(\mathrm{t}, J=7.0 \mathrm{~Hz}, 3 \mathrm{H}) \mathrm{ppm} ;{ }^{13} \mathrm{C} \mathrm{NMR}\left(126 \mathrm{MHz}, \mathrm{CDCl}_{3}\right)$ $\delta=202.6,164.5,161.0,138.0,133.5,129.1$ (2C), 128.4, 128.0, 127.2 124.5, 120.3 (2 C), 112.0, 55.9, 43.9, 31.9, 29.5, 29.4, 29.2, 24.3, 22.7, 14.1 ppm; HRMS (ESI): calcd. for $\mathrm{C}_{23} \mathrm{H}_{30} \mathrm{NO}_{3}[\mathrm{M}+\mathrm{H}]^{+}: 368.2220$ found: 368.2217 .

\section{5 mmol-Scale Reaction of Ni-Catalyzed Cross-Coupling of Phenyl Salicylic Acid Ester with $n$-Octyl Bromide}

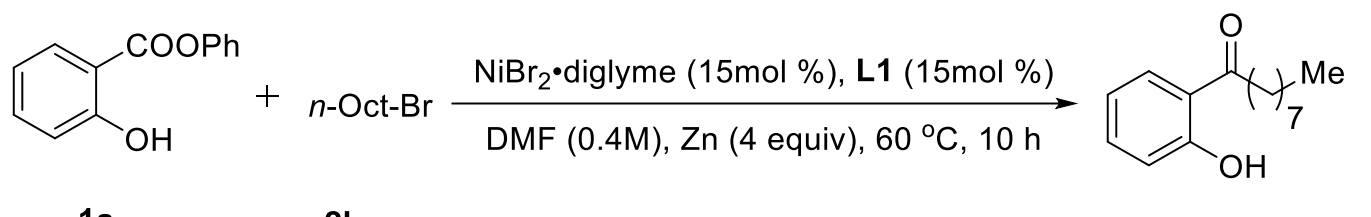

$1 \mathrm{a}$

2b
$3 \mathrm{ab}, 1.04 \mathrm{~g}, 88 \%$ yield

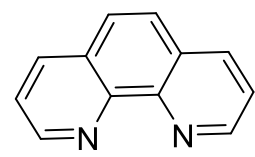

L1

A vial charged with the 1 a (1.1 g, $5.0 \mathrm{mmol}, 1$ equiv), ligand $\mathbf{L 1}$ (135.0 $\mathrm{mg}, 0.75 \mathrm{mmol}, 15 \mathrm{~mol} \%)$ and a stir bar was evacuated and filled with nitrogen (three cycles). In a nitrogen-filled glovebox, $\mathrm{NiBr}_{2} \bullet$ diglyme $(265.0 \mathrm{mg}, 0.75 \mathrm{mmol}, 15 \mathrm{~mol} \%)$ and Zn-powder (1.3 g, $20.0 \mathrm{mmol}, 4.0$ equiv) were added to the mixture. The reaction tube was then sealed and removed from the glove box. Subsequently, DMF $(10 \mathrm{~mL})$ was added to the mixture under nitrogen atmosphere. Then the reaction mixture was heated to $60{ }^{\circ} \mathrm{C}$ with a heating block, before alkyl bormides $2 \mathbf{b}$ ( $\left.1.45 \mathrm{~g}, 7.5 \mathrm{mmol}, 1.5 \mathrm{equiv}\right)$ were added. After stirring at this temperature for $10 \mathrm{~h}$, the mixture was then filtered through a pad of Celite and concentrated under reduced pressure. The residue was purified through column chromatography on silica gel (petroleum ether/ethyl acetate $=10: 1)$ to afford 3ab $(1.04$ g, $88 \%$ yield). 


\section{Control Experiments}<smiles>COc1ccccc1C(=O)Oc1ccccc1</smiles>

11

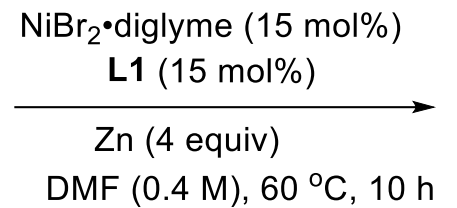

2b<smiles>COc1ccccc1C(=O)[Te]</smiles>

$3 \mathrm{lb}, 0 \%$

A sealed reaction tube charged with the phenolic esters 11 ( $45.7 \mathrm{mg}, 0.2 \mathrm{mmol}, 1$ equiv), ligand $\mathbf{L 1}$ (5.4 mg, $0.03 \mathrm{mmol}, 15$ mol\%) and a stir bar was evacuated and filled with nitrogen (three cycles). In a nitrogen-filled glovebox, $\mathrm{NiBr}_{2} \bullet$ diglyme (10.6 $\mathrm{mg}, 0.03 \mathrm{mmol}, 15 \mathrm{~mol} \%$ ) and $\mathrm{Zn}$-powder ( $52 \mathrm{mg}, 0.8 \mathrm{mmol}, 4.0$ equiv) were added to the mixture. The reaction tube was then sealed and removed from the glove box. Subsequently, DMF $(0.5 \mathrm{~mL})$ was added to the mixture under nitrogen atmosphere. Then the reaction mixture was heated to $60{ }^{\circ} \mathrm{C}$ with a heating block, before 1-bromooctane $2 \mathbf{b}(57.9 \mathrm{mg}, 0.3$ mmol, 1.5 equiv) was added. After stirring at this temperature for $10 \mathrm{~h}$, the mixture was then filtered through a pad of Celite and concentrated under reduced pressure. The residue was subjected to TLC and NMR analysis, indicating that the desired coupling product 3lb was not formed.<smiles>O=C(Oc1ccccc1)c1ccc(O)cc1</smiles>

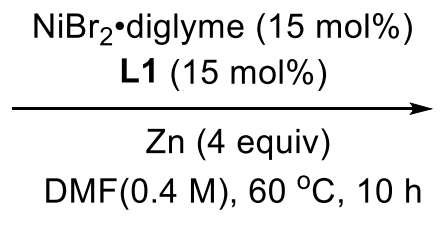<smiles>CC(C)C(=O)c1ccc(O)cc1</smiles>

A sealed reaction tube charged with the phenolic esters $1 \mathbf{m}$ ( $45.7 \mathrm{mg}, 0.2 \mathrm{mmol}, 1$ equiv), ligand $\mathbf{L 1}$ (5.4 mg, $0.03 \mathrm{mmol}, 15$ mol\%) and a stir bar was evacuated and filled with nitrogen (three cycles). In a nitrogen-filled glovebox, $\mathrm{NiBr}_{2} \bullet$ diglyme (10.6 $\mathrm{mg}, 0.03 \mathrm{mmol}, 15 \mathrm{~mol} \%$ ) and $\mathrm{Zn}$-powder ( $52 \mathrm{mg}, 0.8 \mathrm{mmol}, 4.0$ equiv) were added to the mixture. The reaction tube was then sealed and removed from the glove box. Subsequently, DMF $(0.5 \mathrm{~mL})$ was added to the mixture under nitrogen atmosphere. Then the reaction mixture was heated with a heating block, before 1-bromooctane $2 \mathbf{b}(57.9 \mathrm{mg}, 0.3 \mathrm{mmol}, 1.5$ equiv) was added. After stirring at this temperature for $10 \mathrm{~h}$, the mixture was then filtered through a pad of Celite and concentrated under reduced pressure. The residue was subjected to TLC and NMR analysis, indicating that the desired coupling product $3 \mathbf{m b}$ was not formed.<smiles>O=C(Oc1ccccc1)c1ccccc1O</smiles>

$1 \mathrm{a}$

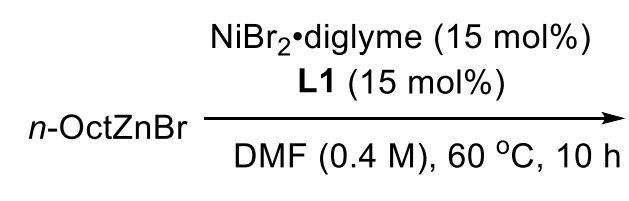

$\operatorname{DMF}(0.4 \mathrm{M}), 60^{\circ} \mathrm{C}, 10 \mathrm{~h}$<smiles>[Y][Y](=O)C(=O)c1ccccc1O</smiles>

$3 \mathrm{ab}, 0 \%$

A sealed reaction tube charged with the phenolic esters 1a ( $42.8 \mathrm{mg}, 0.2 \mathrm{mmol}, 1$ equiv), ligand $\mathbf{L 1}$ (5.4 mg, $0.03 \mathrm{mmol}, 15$ mol\%) and a stir bar was evacuated and filled with nitrogen (three cycles). In a nitrogen-filled glovebox, $\mathrm{NiBr}_{2} \bullet \operatorname{diglyme}(10.6$ $\mathrm{mg}, 0.03 \mathrm{mmol}, 15 \mathrm{~mol} \%$ ) was added to the mixture. The reaction tube was then sealed and removed from the glove box. Subsequently, DMF $(0.5 \mathrm{~mL})$ was added to the mixture under nitrogen atmosphere. Then the reaction mixture was heated to $60{ }^{\circ} \mathrm{C}$ with a heating block, before $n$-OctZnBr ${ }^{17}(0.72 \mathrm{~mL}, 0.83 \mathrm{M}$ in THF, 3 equiv $)$ was added. After stirring at this temperature for $10 \mathrm{~h}$, the mixture was then filtered through a pad of Celite and concentrated under reduced pressure. The residue was subjected to TLC and NMR analysis, indicating that the desired coupling product 3ab was not formed. 


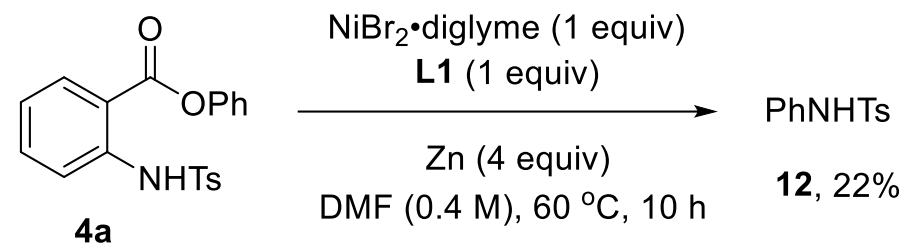

A sealed reaction tube charged with the phenolic esters $4 \mathbf{a}$ (73.5 mg, $0.2 \mathrm{mmol}, 1$ equiv), ligand $\mathbf{L 1}$ (36.0 mg, $0.2 \mathrm{mmol}, 1$ equiv) and a stir bar was evacuated and filled with nitrogen (three cycles). In a nitrogen-filled glovebox, $\mathrm{NiBr}_{2} \bullet \operatorname{diglyme}(71.0$ $\mathrm{mg}, 0.2 \mathrm{mmol}, 1$ equiv) and $\mathrm{Zn}$-powder ( $52 \mathrm{mg}, 0.8 \mathrm{mmol}, 4.0$ equiv) were added to the mixture. The reaction tube was then sealed and removed from the glove box. Subsequently, DMF $(0.5 \mathrm{~mL})$ was added to the mixture under nitrogen atmosphere. After stirring at $60{ }^{\circ} \mathrm{C}$ with a heating block for $10 \mathrm{~h}$, the mixture was then filtered through a pad of Celite and concentrated under reduced pressure. The residue was purified through column chromatography on silica gel (petroleum ether/ethyl acetate $=10: 1)$ to afford PhNHTs (12) as a colorless oil (11 mg, $22 \%$ yield). ${ }^{1} \mathrm{H} \mathrm{NMR}\left(400 \mathrm{MHz}, \mathrm{CDCl}_{3}\right) \delta=7.66(\mathrm{~d}, J=8.2 \mathrm{~Hz}, 2 \mathrm{H})$, 7.24-7.17 (m, 4H), 7.15-7.03 (m, 3H), $6.82(\mathrm{~s}, 1 \mathrm{H}), 2.37$ (s, 3H) ppm; ${ }^{13} \mathrm{C}$ NMR $\left(101 \mathrm{MHz}, \mathrm{CDCl}_{3}\right) \delta=143.9,136.5,136.1,129.6$ (2C), 129.3 (2C), 127.3 (2C), 125.3, 121.6 (2C), 21.5 ppm. HRMS (ESI): calcd. for $\mathrm{C}_{13} \mathrm{H}_{13} \mathrm{NO}_{2} \mathrm{SNa}[\mathrm{M}+\mathrm{Na}]^{+}: 270.0559$ found: 270.0566 .

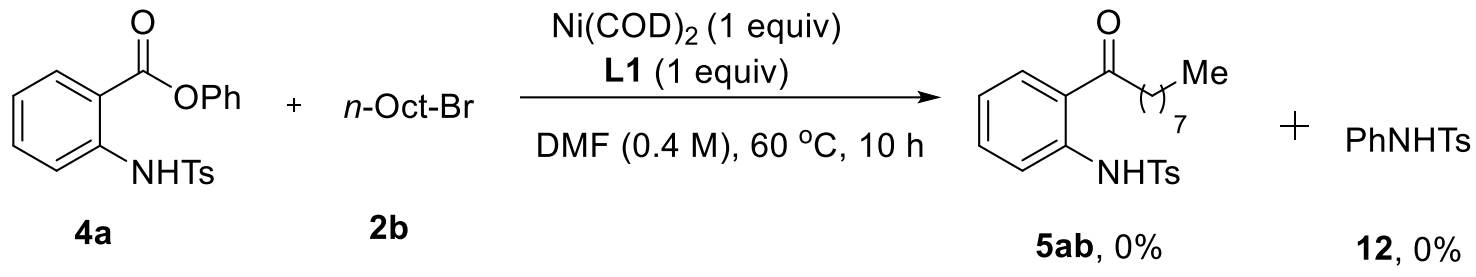

A sealed reaction tube charged with the phenolic esters $4 \mathbf{a}(73.5 \mathrm{mg}, 0.2 \mathrm{mmol}, 1$ equiv), ligand $\mathbf{L 1}$ (36.0 $\mathrm{mg}, 0.2 \mathrm{mmol}, 1$ equiv) and a stir bar was evacuated and filled with nitrogen (three cycles). In a nitrogen-filled glovebox, $\mathrm{Ni}(\mathrm{COD})_{2}(55.0 \mathrm{mg}$, $0.2 \mathrm{mmol}, 1$ equiv) was added to the mixture. The reaction tube was then sealed and removed from the glove box. Subsequently, DMF (0.5 mL) and 1-bromooctane $2 \mathbf{b}(57.9 \mathrm{mg}, 0.3 \mathrm{mmol}, 1.5$ equiv) were added to the mixture under nitrogen atmosphere. After stirring at $60{ }^{\circ} \mathrm{C}$ with a heating block for $10 \mathrm{~h}$, the mixture was then filtered through a pad of Celite and concentrated under reduced pressure. The residue was subjected to TLC and NMR analysis, indicating that neither of the desired coupling product $5 \mathbf{a b}$ and PhNHTs was formed.

\section{Radical Clock Experiment}
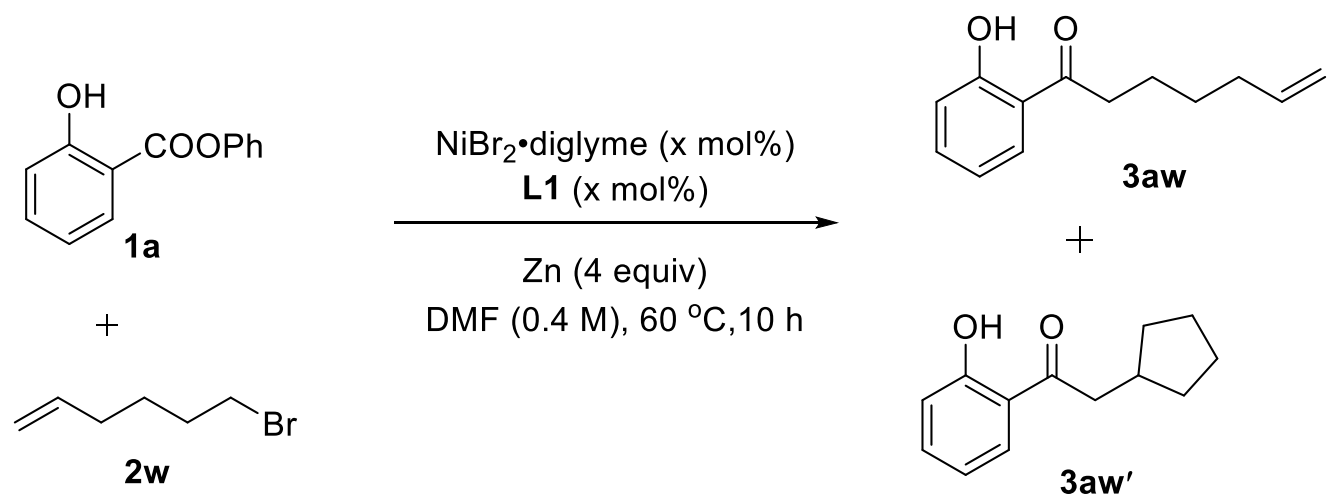


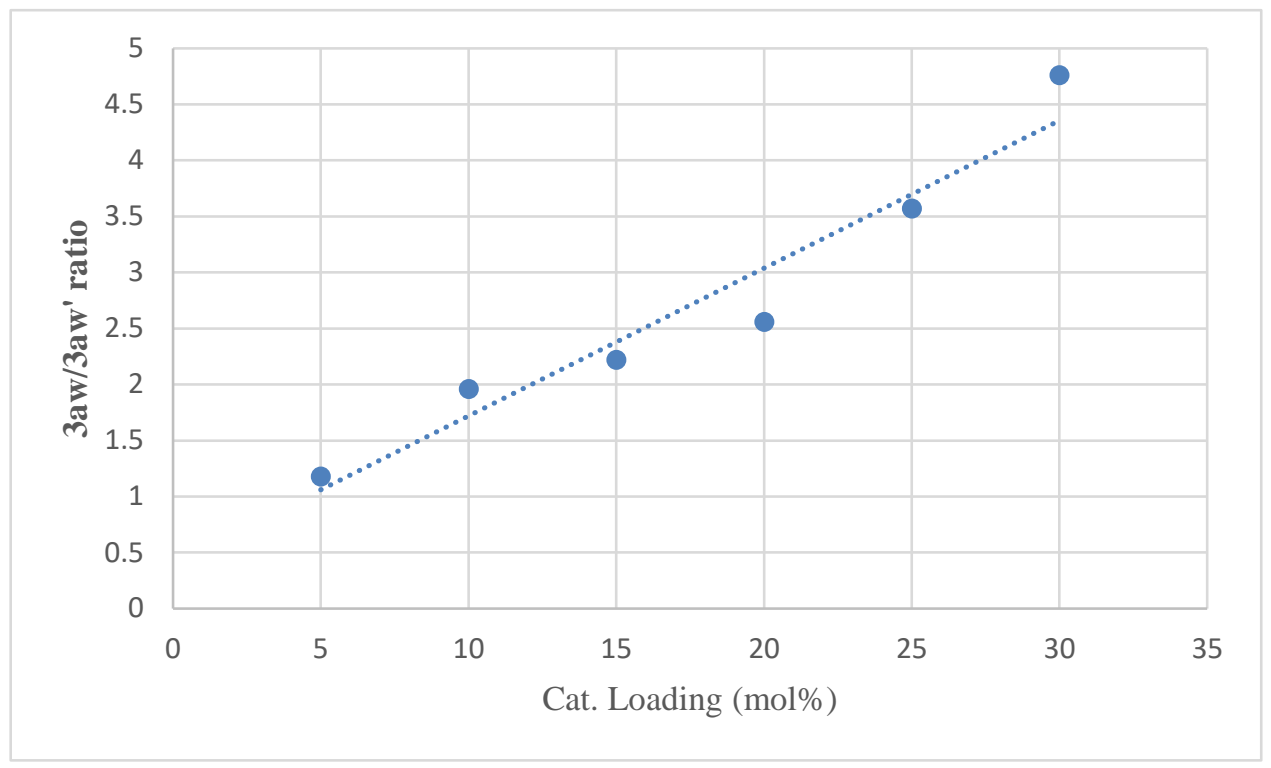

A sealed reaction tube charged with the phenolic esters 1a ( $42.8 \mathrm{mg}, 0.2 \mathrm{mmol}, 1$ equiv), ligand $\mathbf{L 1}$ (1.8 $\mathrm{mg}, 0.015 \mathrm{mmol}, 5$ mol\%) and a stir bar was evacuated and filled with nitrogen (three cycles). In a nitrogen-filled glovebox, $\mathrm{NiBr}_{2} \bullet \operatorname{diglyme}(3.5$ $\mathrm{mg}, 0.015 \mathrm{mmol}, 5 \mathrm{~mol} \%$ ) and $\mathrm{Zn}$-powder ( $52 \mathrm{mg}, 0.8 \mathrm{mmol}, 4.0$ equiv) were added to the mixture. The reaction tube was then sealed and removed from the glove box. Subsequently, DMF $(0.5 \mathrm{~mL})$ was added to the mixture under nitrogen atmosphere. Then the reaction mixture was heated to $60{ }^{\circ} \mathrm{C}$ with a heating block, before 1-bromooctane $2 \mathbf{w}$ ( $48.6 \mathrm{mg}, 0.3$ mmol, 1.5 equiv) was added. After stirring at this temperature for $10 \mathrm{~h}$, the mixture was then filtered through a pad of Celite and concentrated under reduced pressure. The residue was purified through column chromatography on silica gel (petroleum ether) to afford the corresponding products 3aw:3aw'=1:0.85.

A sealed reaction tube charged with the phenolic esters 1a ( $42.8 \mathrm{mg}, 0.2 \mathrm{mmol}, 1$ equiv), ligand $\mathbf{L} 1$ ( $3.6 \mathrm{mg}, 0.02 \mathrm{mmol}, 10$ mol\%) and a stir bar was evacuated and filled with nitrogen (three cycles). In a nitrogen-filled glovebox, $\mathrm{NiBr}_{2} \bullet \operatorname{diglyme}(7.0$ $\mathrm{mg}, 0.02 \mathrm{mmol}, 10 \mathrm{~mol} \%$ ) and $\mathrm{Zn}$-powder ( $52 \mathrm{mg}, 0.8 \mathrm{mmol}, 4.0$ equiv) were added to the mixture. The reaction tube was then sealed and removed from the glove box. Subsequently, DMF $(0.5 \mathrm{~mL})$ was added to the mixture under nitrogen atmosphere. Then the reaction mixture was heated to $60{ }^{\circ} \mathrm{C}$ with a heating block, before 1-bromooctane $2 \mathbf{w}$ ( $48.6 \mathrm{mg}, 0.3$ mmol, 1.5 equiv) was added. After stirring at this temperature for $10 \mathrm{~h}$, the mixture was then filtered through a pad of Celite and concentrated under reduced pressure. The residue was purified through column chromatography on silica gel (petroleum ether) to afford the corresponding products 3aw:3aw'= 1:0.51.

A sealed reaction tube charged with the phenolic esters 1a ( $42.8 \mathrm{mg}, 0.2 \mathrm{mmol}, 1$ equiv), ligand $\mathbf{L 1}$ (5.4mg, $0.03 \mathrm{mmol}, 15$ mol\%) and a stir bar was evacuated and filled with nitrogen (three cycles). In a nitrogen-filled glovebox, $\mathrm{NiBr}_{2} \bullet$ diglyme (10.6 $\mathrm{mg}, 0.015 \mathrm{mmol}, 15 \mathrm{~mol} \%$ ) and $\mathrm{Zn}$-powder (52 $\mathrm{mg}, 0.8 \mathrm{mmol}, 4.0$ equiv) were added to the mixture. The reaction tube was then sealed and removed from the glove box. Subsequently, DMF $(0.5 \mathrm{~mL})$ was added to the mixture under nitrogen atmosphere. Then the reaction mixture was heated to $60{ }^{\circ} \mathrm{C}$ with a heating block, before 1-bromooctane $2 \mathbf{w}$ ( $48.6 \mathrm{mg}, 0.3$ mmol, 1.5 equiv) was added. After stirring at this temperature for $10 \mathrm{~h}$, the mixture was then filtered through a pad of Celite and concentrated under reduced pressure. The residue was purified through column chromatography on silica gel (petroleum ether) to afford the corresponding products 3aw:3aw'= 1:0.45.

A sealed reaction tube charged with the phenolic esters 1a ( $42.8 \mathrm{mg}, 0.2 \mathrm{mmol}, 1$ equiv), ligand $\mathbf{L 1}$ (7.2 $\mathrm{mg}, 0.04 \mathrm{mmol}, 20$ mol\%) and a stir bar was evacuated and filled with nitrogen (three cycles). In a nitrogen-filled glovebox, $\mathrm{NiBr}_{2} \bullet \operatorname{diglyme}(14.0$ $\mathrm{mg}, 0.04 \mathrm{mmol}, 20 \mathrm{~mol} \%$ ) and $\mathrm{Zn}$-powder ( $52 \mathrm{mg}, 0.8 \mathrm{mmol}, 4.0$ equiv) were added to the mixture. The reaction tube was then sealed and removed from the glove box. Subsequently, DMF $(0.5 \mathrm{~mL})$ was added to the mixture under nitrogen 
atmosphere. Then the reaction mixture was heated to $60{ }^{\circ} \mathrm{C}$ with a heating block, before 1-bromooctane $2 \mathbf{w}$ ( $48.6 \mathrm{mg}, 0.3$ mmol, 1.5 equiv) was added. After stirring at this temperature for $10 \mathrm{~h}$, the mixture was then filtered through a pad of Celite and concentrated under reduced pressure. The residue was purified through column chromatography on silica gel (petroleum ether) to afford the corresponding products 3aw:3aw'= 1:0.39.

A sealed reaction tube charged with the phenolic esters 1a ( $42.8 \mathrm{mg}, 0.2 \mathrm{mmol}, 1$ equiv), ligand $\mathbf{L} 1$ ( $9.0 \mathrm{mg}, 0.05 \mathrm{mmol}, 25$ mol\%) and a stir bar was evacuated and filled with nitrogen (three cycles). In a nitrogen-filled glovebox, $\mathrm{NiBr}_{2} \bullet \operatorname{diglyme}(17.5$ $\mathrm{mg}, 0.015 \mathrm{mmol}, 25 \mathrm{~mol} \%$ ) and $\mathrm{Zn}$-powder (52 $\mathrm{mg}, 0.8 \mathrm{mmol}, 4.0$ equiv) were added to the mixture. The reaction tube was then sealed and removed from the glove box. Subsequently, DMF $(0.5 \mathrm{~mL})$ was added to the mixture under nitrogen atmosphere. Then the reaction mixture was heated to $60{ }^{\circ} \mathrm{C}$ with a heating block, before 1-bromooctane $\mathbf{2 w}$ ( $48.6 \mathrm{mg}, 0.3$ mmol, 1.5 equiv) was added. After stirring at this temperature for $10 \mathrm{~h}$, the mixture was then filtered through a pad of Celite and concentrated under reduced pressure. The residue was purified through column chromatography on silica gel (petroleum ether) to afford the corresponding products 3aw:3aw'= 1:0.28.

A sealed reaction tube charged with the phenolic esters 1a $(42.8 \mathrm{mg}, 0.2 \mathrm{mmol}, 1$ equiv), ligand $\mathbf{L 1}$ (10.8 $\mathrm{mg}, 0.06 \mathrm{mmol}, 30$ mol\%) and a stir bar was evacuated and filled with nitrogen (three cycles). In a nitrogen-filled glovebox, $\mathrm{NiBr}_{2} \bullet$ diglyme (121.0 mg, $0.06 \mathrm{mmol}, 30 \mathrm{~mol} \%$ ) and Zn-powder (52 mg, $0.8 \mathrm{mmol}, 4.0$ equiv) were added to the mixture. The reaction tube was then sealed and removed from the glove box. Subsequently, DMF $(0.5 \mathrm{~mL})$ was added to the mixture under nitrogen atmosphere. Then the reaction mixture was heated to $60{ }^{\circ} \mathrm{C}$ with a heating block, before 1-bromooctane $\mathbf{2 w}$ ( $48.6 \mathrm{mg}, 0.3$ mmol, 1.5 equiv) was added. After stirring at this temperature for $10 \mathrm{~h}$, the mixture was then filtered through a pad of Celite and concentrated under reduced pressure. The residue was purified through column chromatography on silica gel (petroleum ether) to afford the corresponding products 3aw:3aw'= 1:0.21.

Conpound 3aw: ${ }^{1} \mathrm{H}$ NMR $\left(400 \mathrm{MHz}, \mathrm{CDCl}_{3}\right) \delta=12.38(\mathrm{~s}, 1 \mathrm{H}), 7.76(\mathrm{dd}, J=8.1,1.8 \mathrm{~Hz}, 1 \mathrm{H}), 7.51-7.39(\mathrm{~m}, 1 \mathrm{H}), 6.98(\mathrm{dd}, J$ $=8.3,1.2 \mathrm{~Hz}, 1 \mathrm{H}), 6.92-6.85(\mathrm{~m}, 1 \mathrm{H}), 5.89-5.74(\mathrm{~m}, 1 \mathrm{H}), 5.09-4.85(\mathrm{~m}, 2 \mathrm{H}), 3.05-2.91(\mathrm{~m}, 2 \mathrm{H}), 2.23-2.02(\mathrm{~m}, 2 \mathrm{H}), 1.83-$ $1.69(\mathrm{~m}, 2 \mathrm{H}), 1.55-1.44(\mathrm{~m}, 2 \mathrm{H}) \mathrm{ppm}$.

Conpound 3aw': ' $\mathrm{H}$ NMR (400 MHz, $\left.\mathrm{CDCl}_{3}\right) \delta=12.40(\mathrm{~s}, 1 \mathrm{H}), 7.76(\mathrm{dd}, J=8.1,1.8 \mathrm{~Hz}, 1 \mathrm{H}), 7.51-7.39(\mathrm{~m}, 1 \mathrm{H}), 6.98(\mathrm{dd}, J$ $=8.3,1.2 \mathrm{~Hz}, 1 \mathrm{H}), 6.92-6.85(\mathrm{~m}, 1 \mathrm{H}), 3.05-2.91(\mathrm{~m}, 2 \mathrm{H}), 2.23-2.02(\mathrm{~m}, 1 \mathrm{H}), 1.83-1.54(\mathrm{~m}, 8 \mathrm{H}) \mathrm{ppm}$.

\section{References}

1. N. A. Serratore, C. B. Anderson, G. B. Frost, T. G. Hoang, S. J. Underwood, P. M. Gemmel, M. A. Hardy, C. J. Douglas, J. Am. Chem. Soc.2018, 140, 10025-10033

2. D. Alpers, K. P. Cole, C. R. J. Stephenson, Angew.Chem. Int.Ed. 2018, 57,12167-12170.

3. M. Shang, S.-Z. Sun, H.-X. Dai, J.-Q. Yu, J. Am. Chem. Soc.2014, 136, 3354-3357.

4. D. Gartner, A. L. Stein, S. Grupe, J. Arp, A. J. von Wangelin, Angew.Chem. Int.Ed. 2015, 54, 10545-10549.

5. F. Brunel, C. Lautard, F, Garzino, S. Giorgio, J. M. Raimundo, J. M. Bolla, M. Camplo, Bioorg. Med. Chem. Lett. 2016, $2,3770-3773$.

6. Y. Liu, L. Ji, M. Eno, S. Kudalkar, A.-L. Li, M. Schimpgen, O. Benchama, P. Morales, S. Xu, D. Hurst, S. Wu, K. A. Mohammad, J. T. Wood, N. Zvonok, D. P. Papahatjis, H. Zhou, C. Honrao, K. Mackie, P. Reggio, A. G. Hohmann, L. J. Marnett, A. Makriyannis, S. P. Nikas, J. Med. Chem. 2018, 61, 8639-8657.

7. D. Ding, H. Dong, C. Wang, iScience 2020, 23, 101017.

8. Z. Lin, Y. Lan, C. Wang, Org. Lett. 2019, 21, 8316-8322.

9. T. C. Atack, S. P. Cook, J. Am. Chem. Soc. 2016, 138, 6139-6142.

10. M. Von Delius, C. M. Le, V. M. Dong, J. Am. Chem. Soc.2012, 134, 15022-15032.

11. A. D. Alorati, A. D. Gibb, P. R. Mullens, Gavin. W. Stewart, Org. Proc. Res. Dev. 2012, 16, 1947-1952. 
12. M. Vimal, U. Pathak, A. K. Halve, Synth. Commun. 2019, 49, 2805-2814.

13. R. Perrone, F. Berardi, N. A. Colabufo, E. Lacivita, M. Leopoldo, V. Tortorella, J. Med. Chem. 2003, 46, 646-649.

14. L. Hu, Y. Zhang, Q.-W. Zhang, Q. Yin, X. Zhang, Angew. Chem. Int. Ed. 2020, 59, 5321-5325.

15. P. G. Cittani, G. Mastropietro, E. Morera, G. Ortar, Tetrahedron Lett. 1993, 34, 3763-3766.

16. T. B. Halima, J. K. Vandavasi, M. Shkoor, S. G. Newman, ACS Catal. 2017, 7, 2176-2180.

17. J. Pan, M. Zhang, S. Zhang, Org. Biomol. Chem. 2012, 10, 1060-1067. 


\section{NMR-Spectra}

${ }^{1} \mathrm{H}$ NMR-spectrum $\left(500 \mathrm{MHz}, \mathrm{CDCl}_{3}\right)$ of $\mathbf{1 i}$

屏品

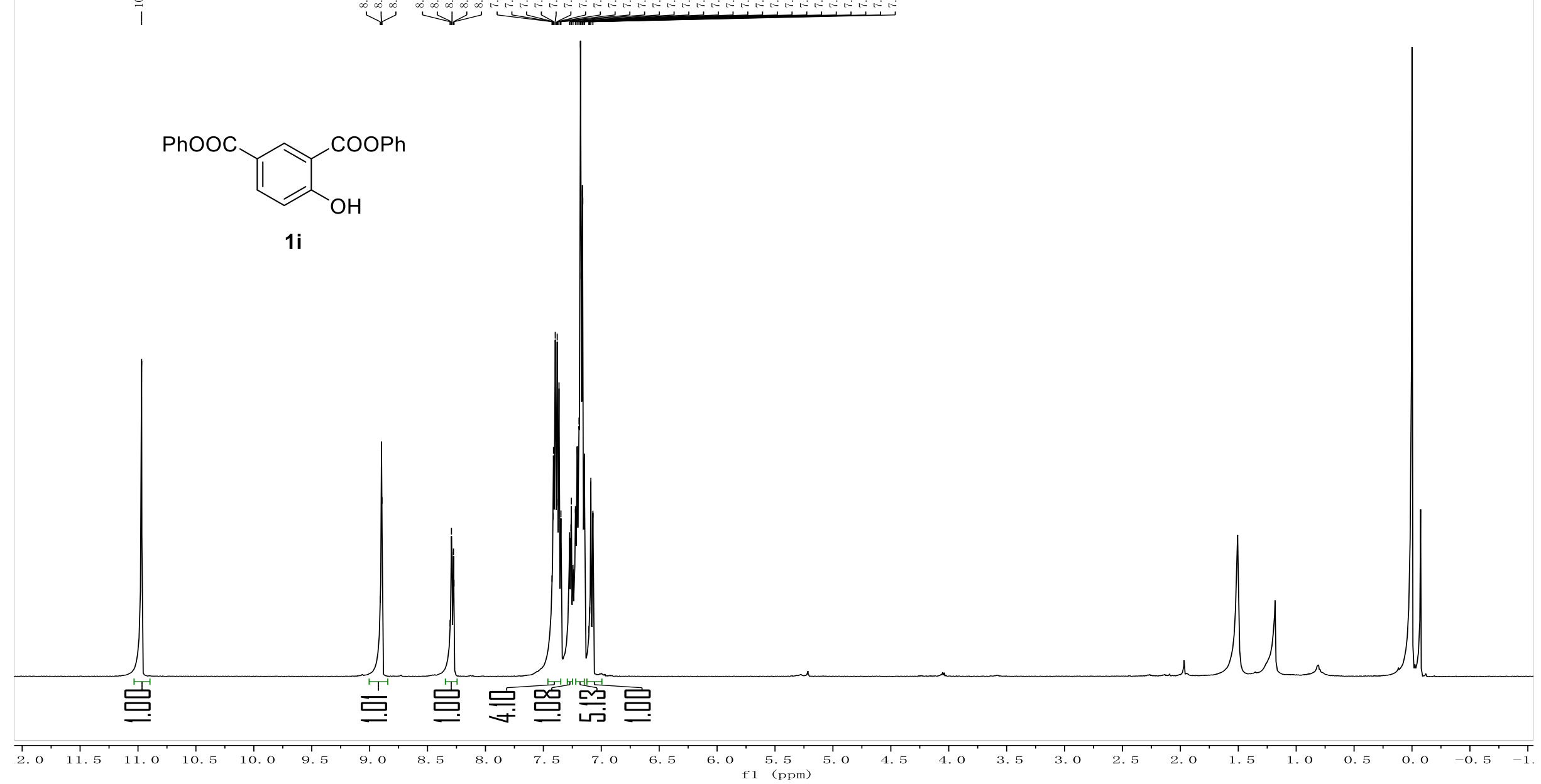

$1 \mathrm{i}$ 
${ }^{13} \mathrm{C}$ NMR-spectrum $\left(126 \mathrm{MHz}, \mathrm{CDCl}_{3}\right)$ of $\mathbf{1 i}$
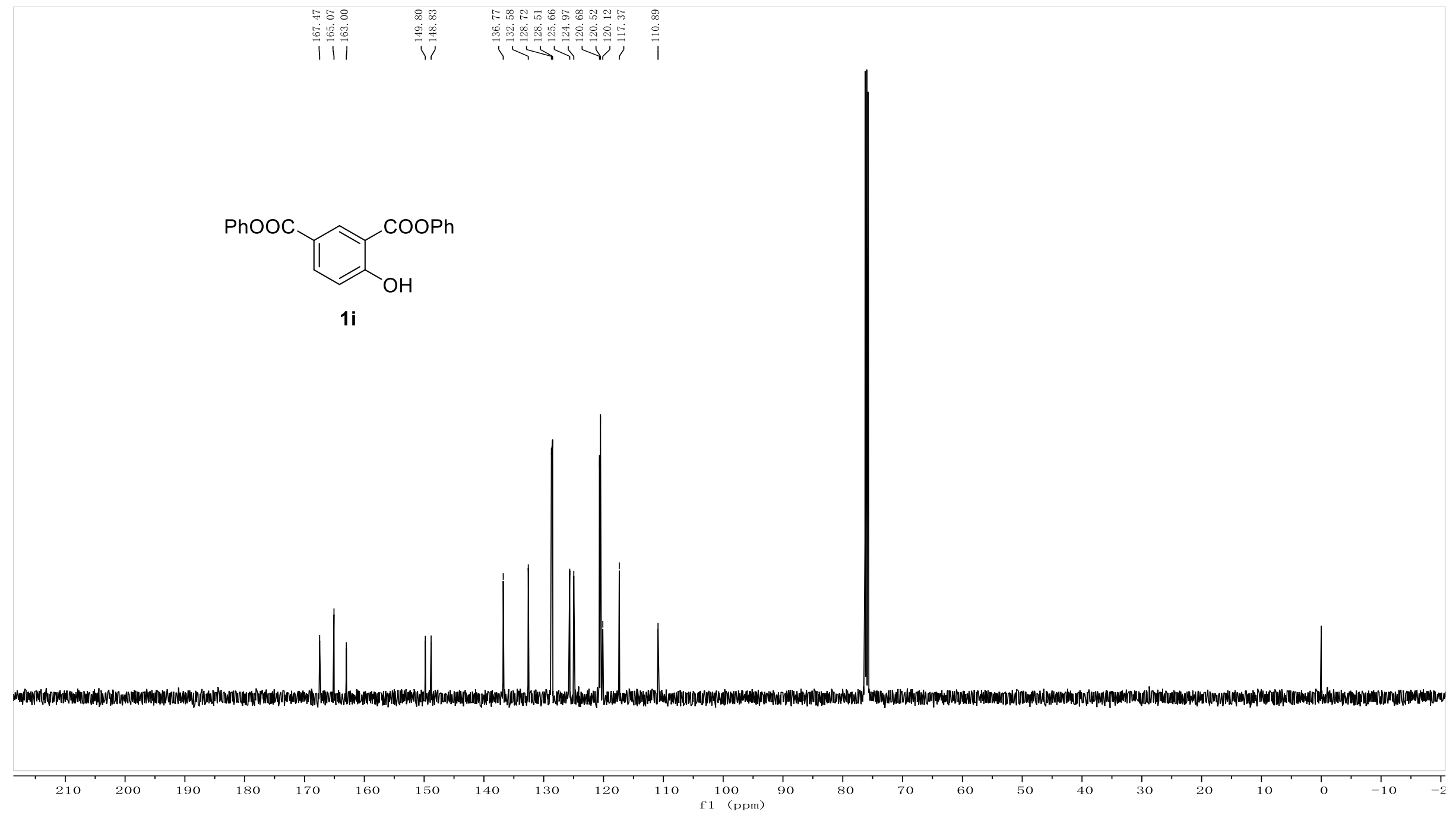
${ }^{1} \mathrm{H}$ NMR-spectrum $\left(400 \mathrm{MHz}, \mathrm{CDCl}_{3}\right)$ of $\mathbf{1} \mathbf{j}$

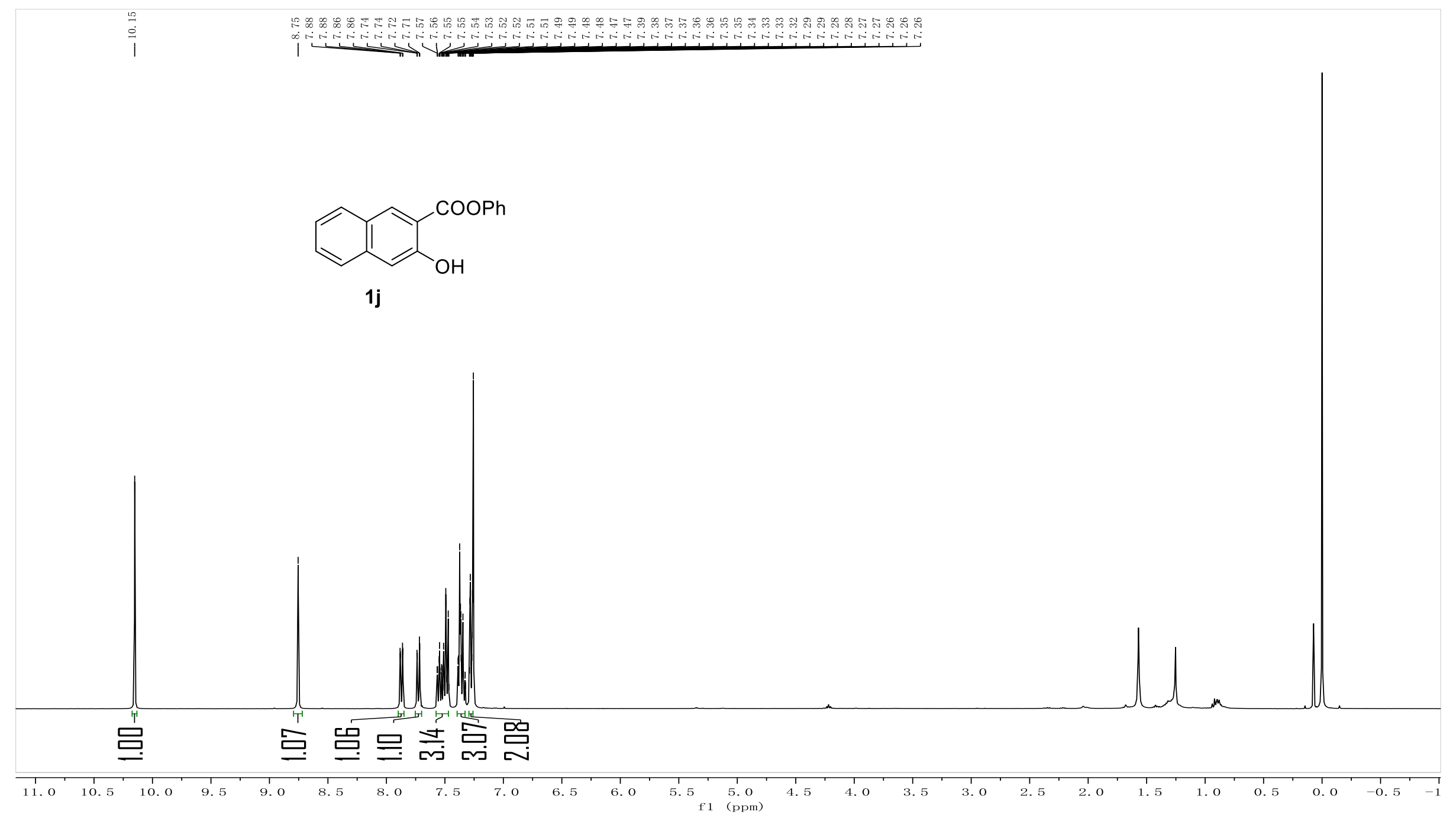


${ }^{13} \mathrm{C}$ NMR-spectrum $\left(101 \mathrm{MHz}, \mathrm{CDCl}_{3}\right)$ of $\mathbf{1 j}$
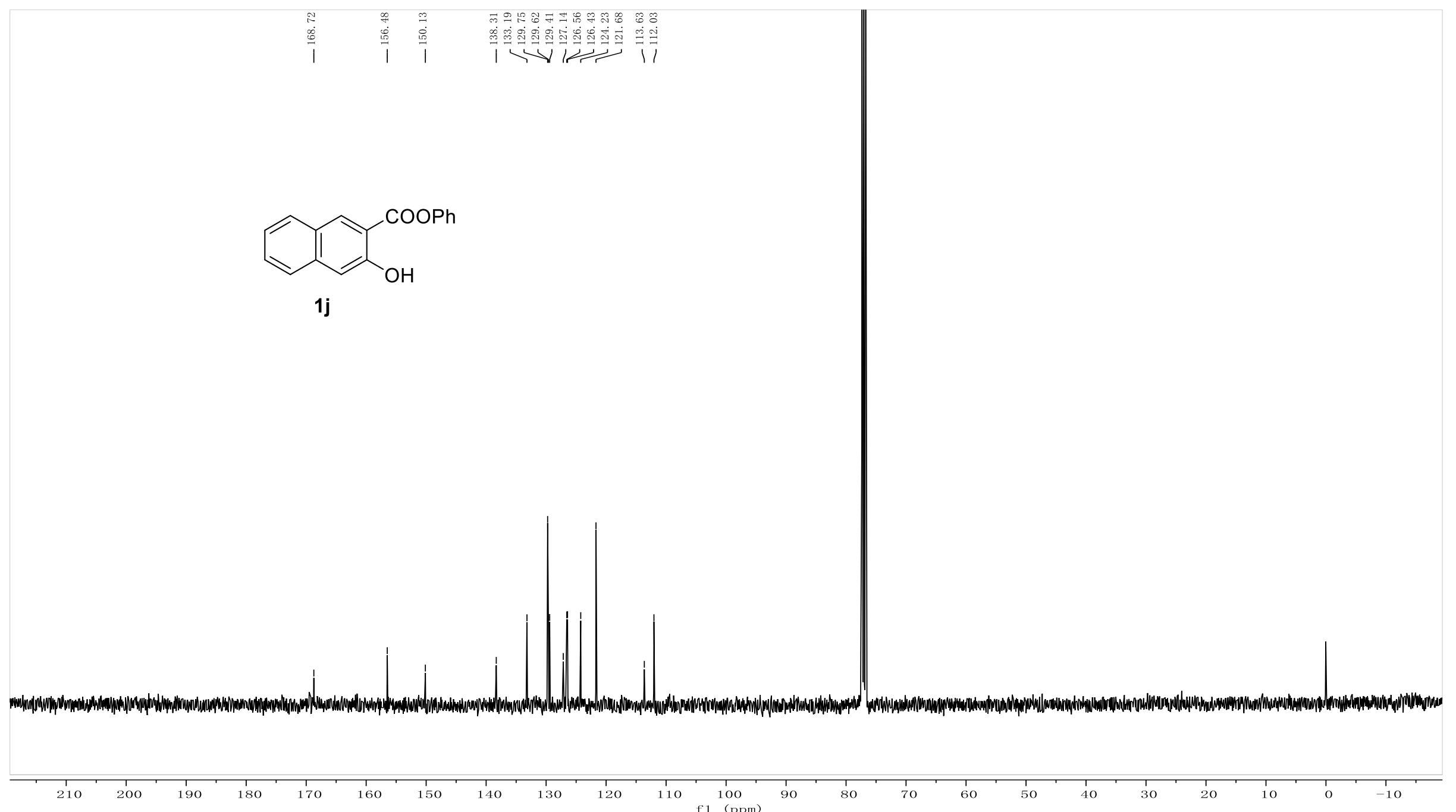
${ }^{1} \mathrm{H}$ NMR-spectrum (400 MHz, DMSO- $\left.d_{6}\right)$ of S-1

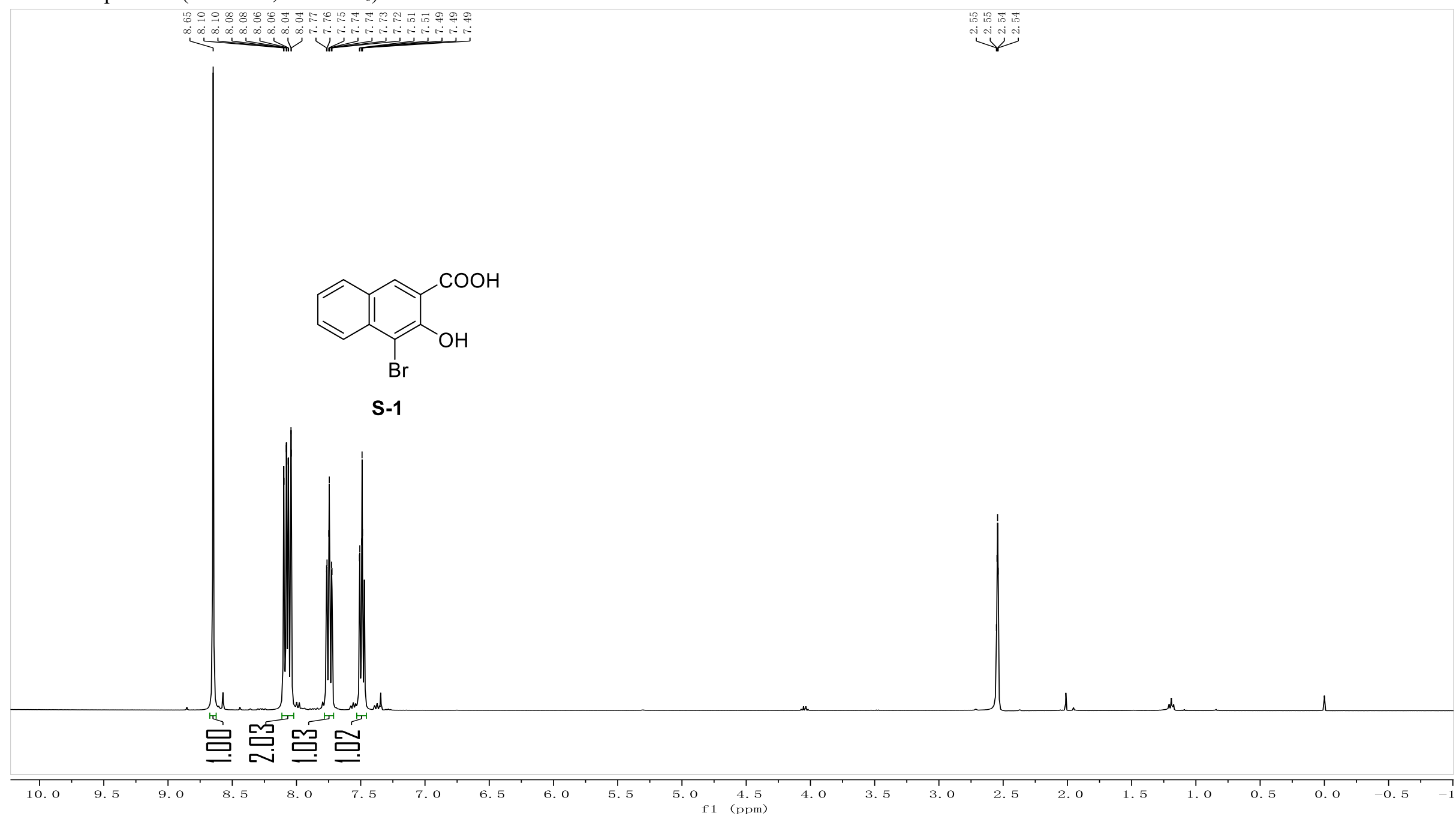


${ }^{13} \mathrm{C}$ NMR-spectrum (101 MHz, DMSO- $\left.d_{6}\right)$ of $\mathbf{S - 1}$

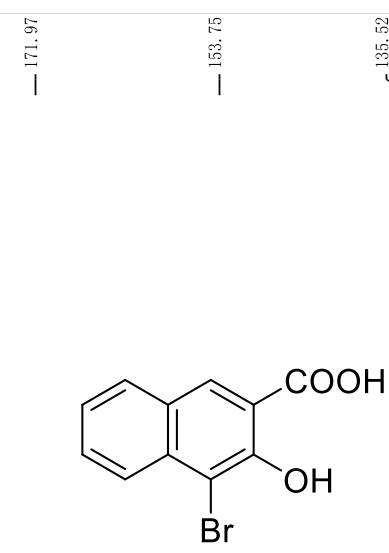

S-1

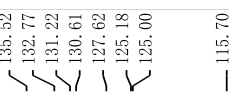

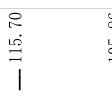

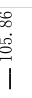

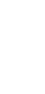

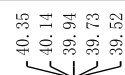

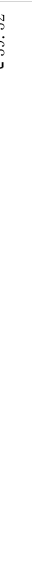


${ }^{1} \mathrm{H}$ NMR-spectrum $\left(400 \mathrm{MHz}, \mathrm{CDCl}_{3}\right)$ of $\mathbf{S}-\mathbf{2}$

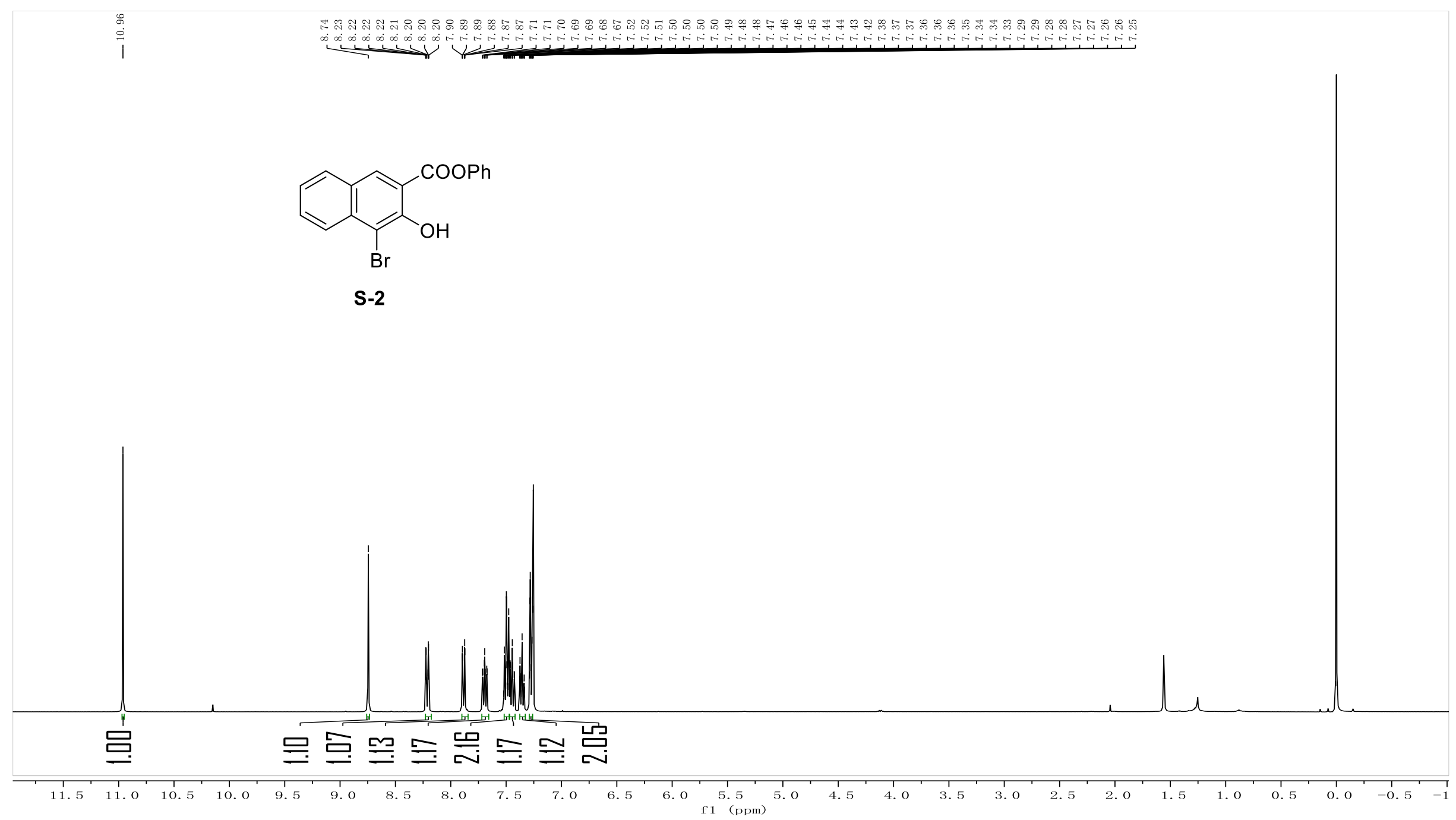


${ }^{13} \mathrm{C}$ NMR-spectrum $\left(101 \mathrm{MHz}, \mathrm{CDCl}_{3}\right)$ of $\mathbf{S - 2}$
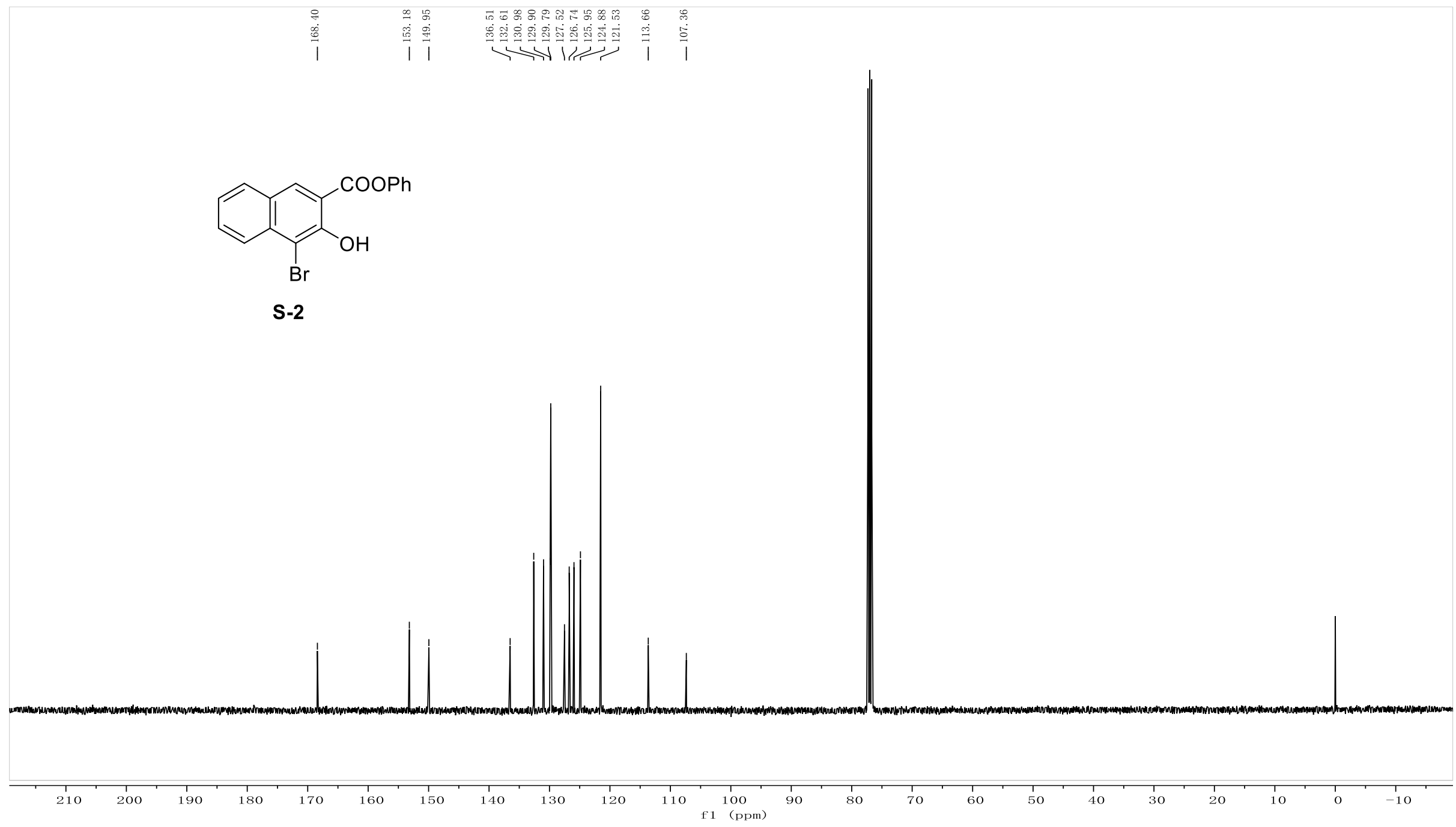
${ }^{1} \mathrm{H}$ NMR-spectrum $\left(400 \mathrm{MHz}, \mathrm{CDCl}_{3}\right)$ of $\mathbf{1 k}$

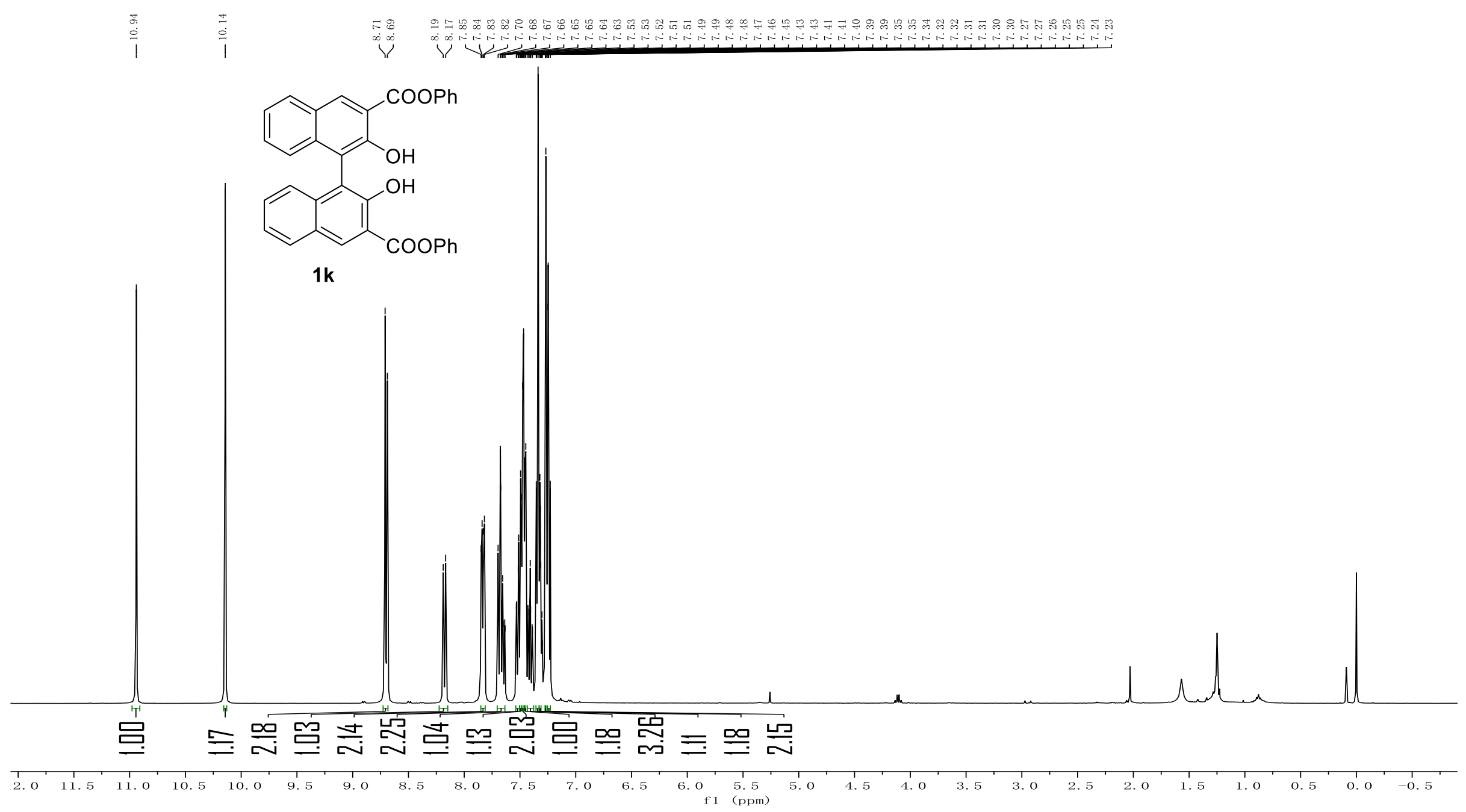


${ }^{13} \mathrm{C}$ NMR-spectrum $\left(126 \mathrm{MHz}, \mathrm{CDCl}_{3}\right)$ of $\mathbf{1} \mathbf{k}$
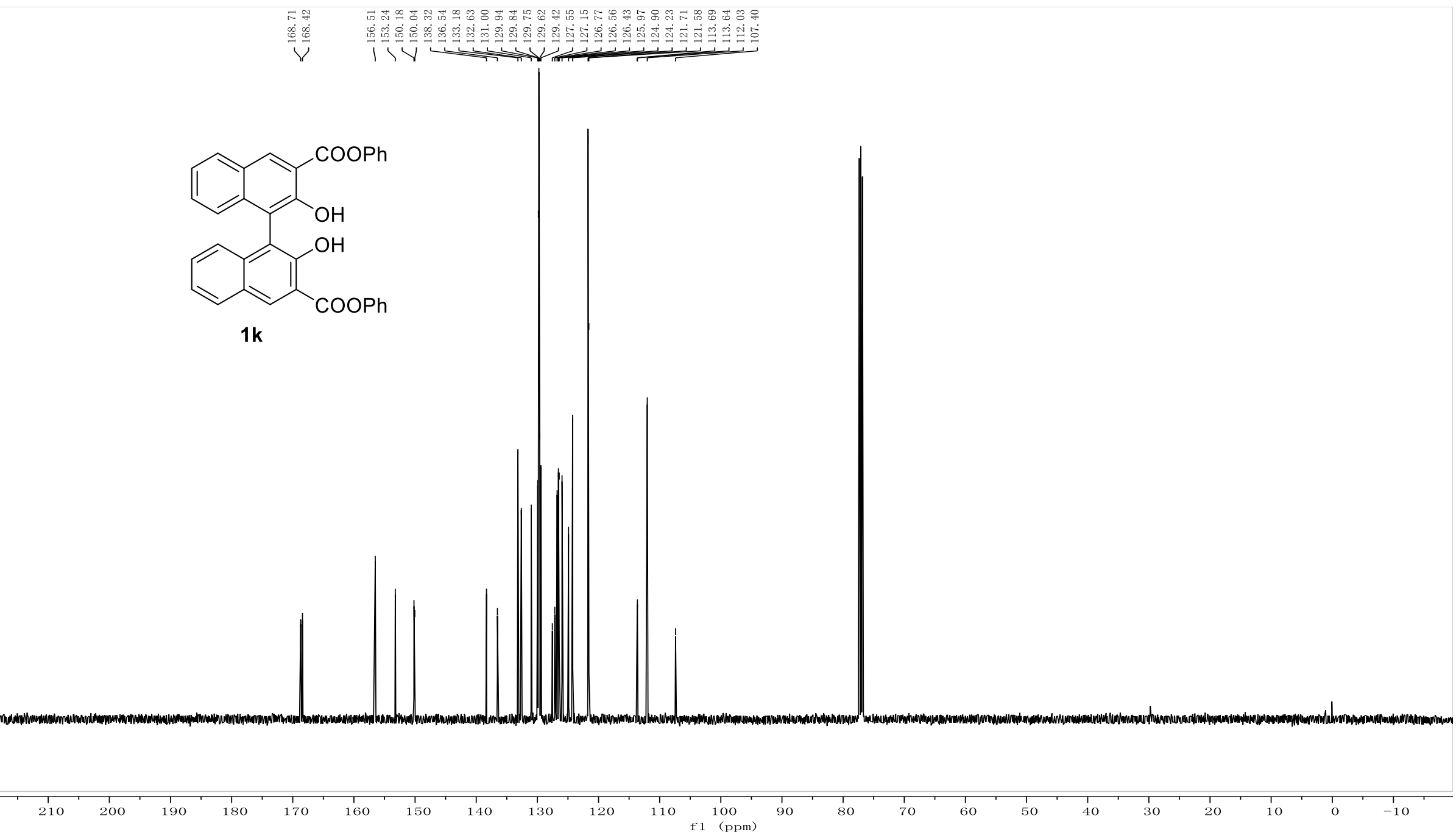
${ }^{1} \mathrm{H}$ NMR-spectrum $\left(400 \mathrm{MHz}, \mathrm{CDCl}_{3}\right)$ of $\mathbf{S - 3 c}$

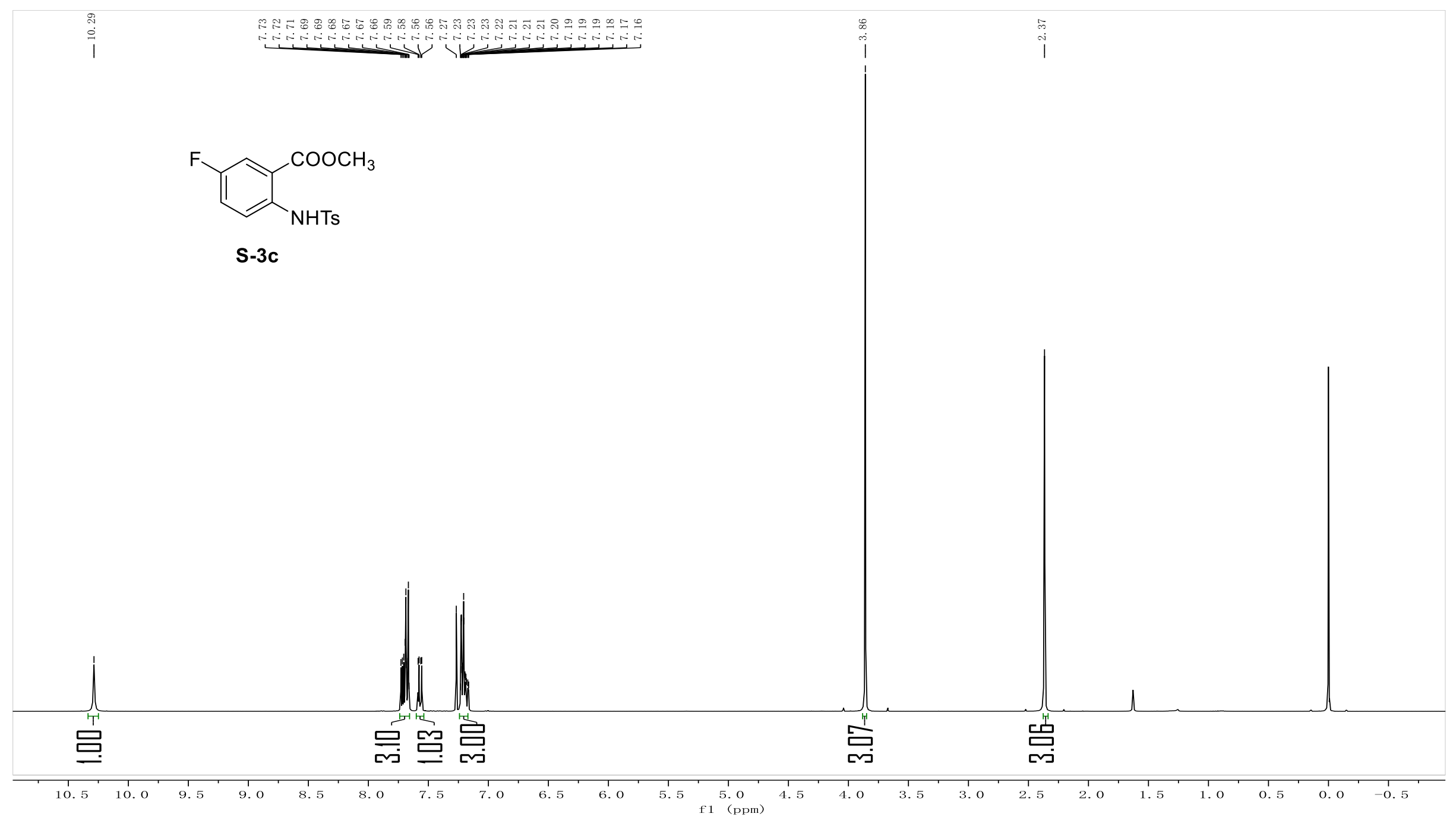


${ }^{13} \mathrm{C}$ NMR-spectrum $\left(101 \mathrm{MHz}, \mathrm{CDCl}_{3}\right)$ of $\mathbf{S - 3 c}$
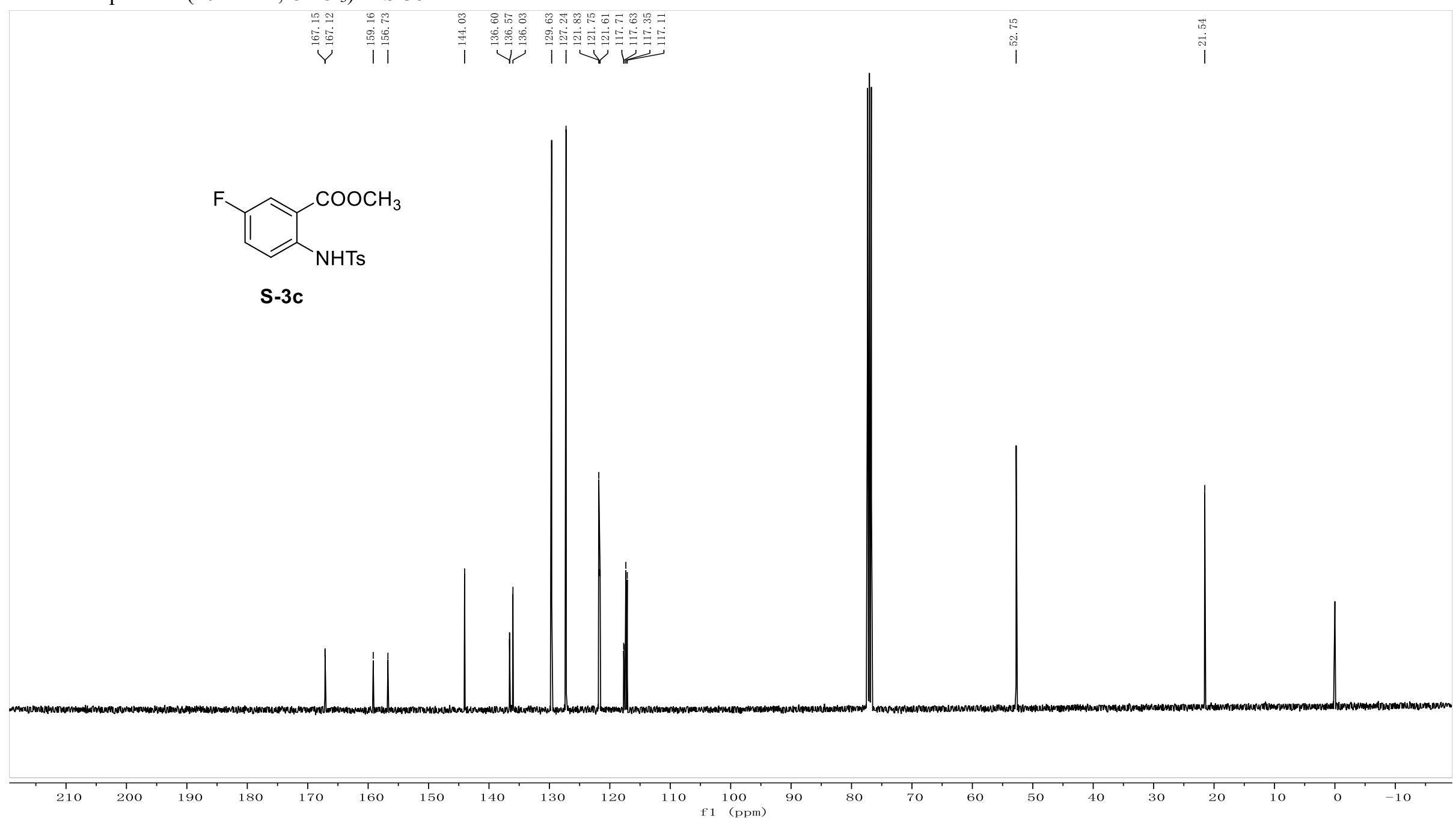
${ }^{19}$ F NMR-spectrum $\left(376 \mathrm{MHz}, \mathrm{CDCl}_{3}\right)$ of $\mathbf{S - 3 c}$

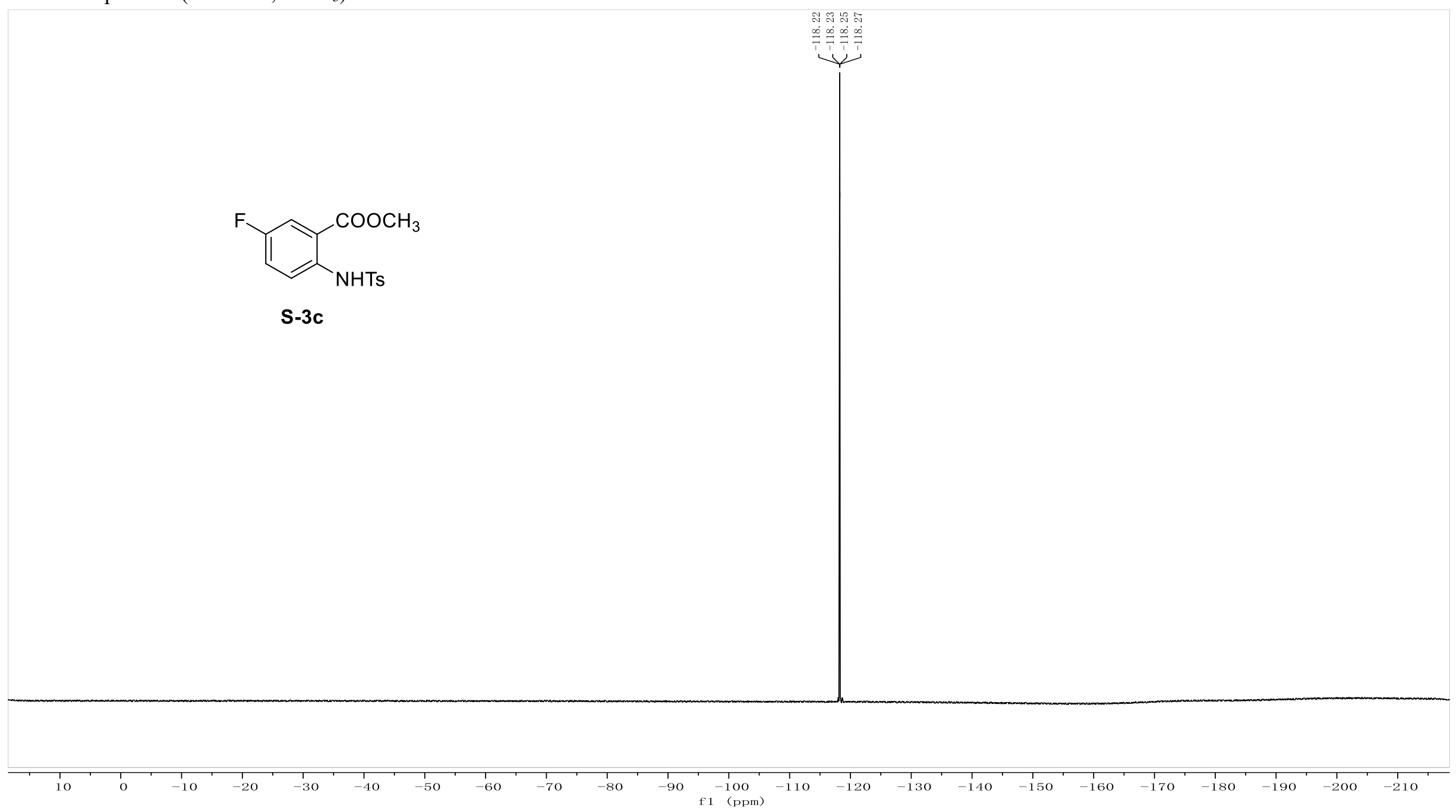


${ }^{1} \mathrm{H}$ NMR-spectrum $\left(400 \mathrm{MHz}, \mathrm{CDCl}_{3}\right)$ of $\mathbf{S - 3 d}$

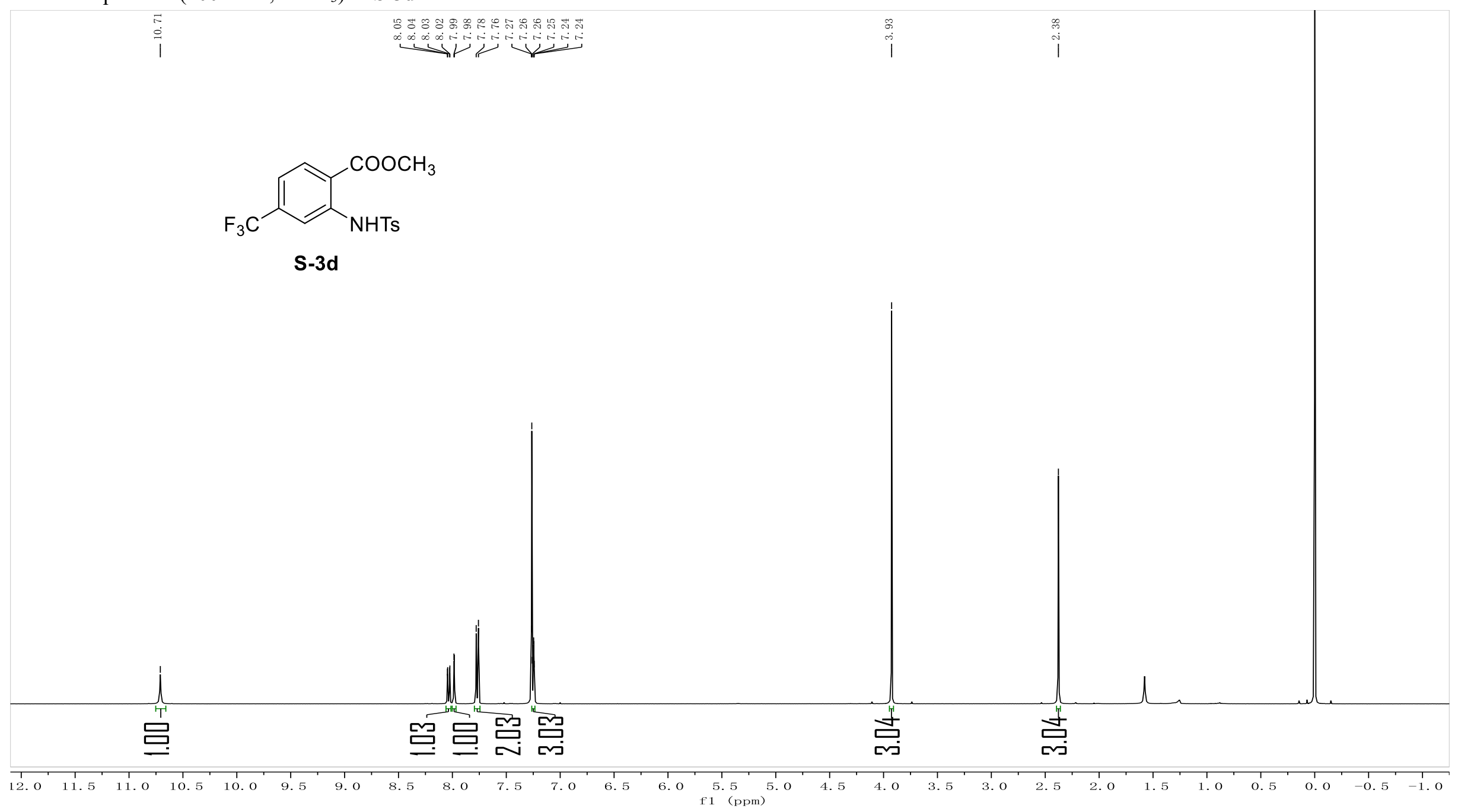


${ }^{13} \mathrm{C}$ NMR-spectrum $\left(101 \mathrm{MHz}, \mathrm{CDCl}_{3}\right)$ of $\mathbf{S}-\mathbf{3 d}$
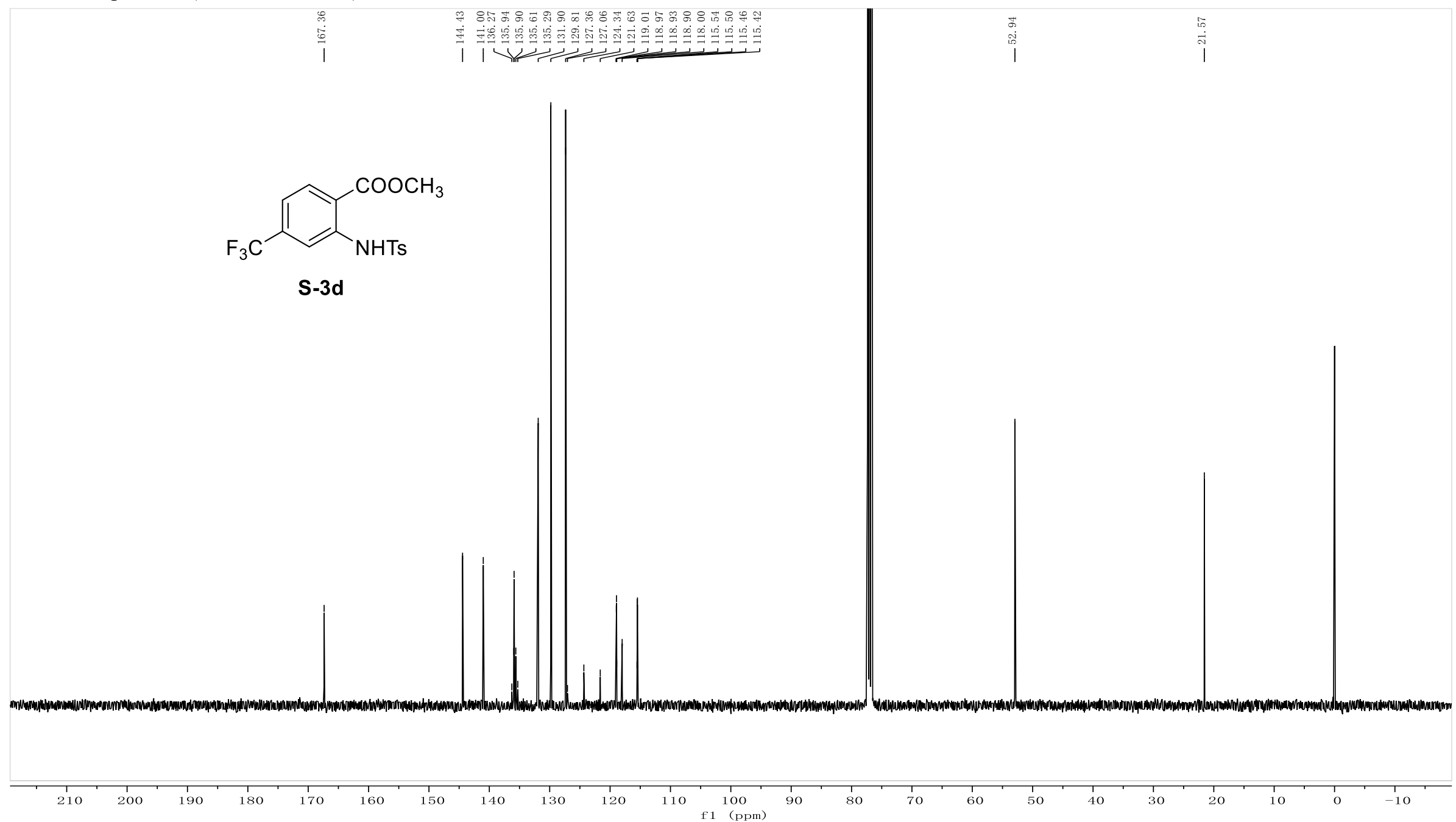
${ }^{19}$ F NMR-spectrum $\left(376 \mathrm{MHz}, \mathrm{CDCl}_{3}\right)$ of $\mathbf{S - 3 d}$

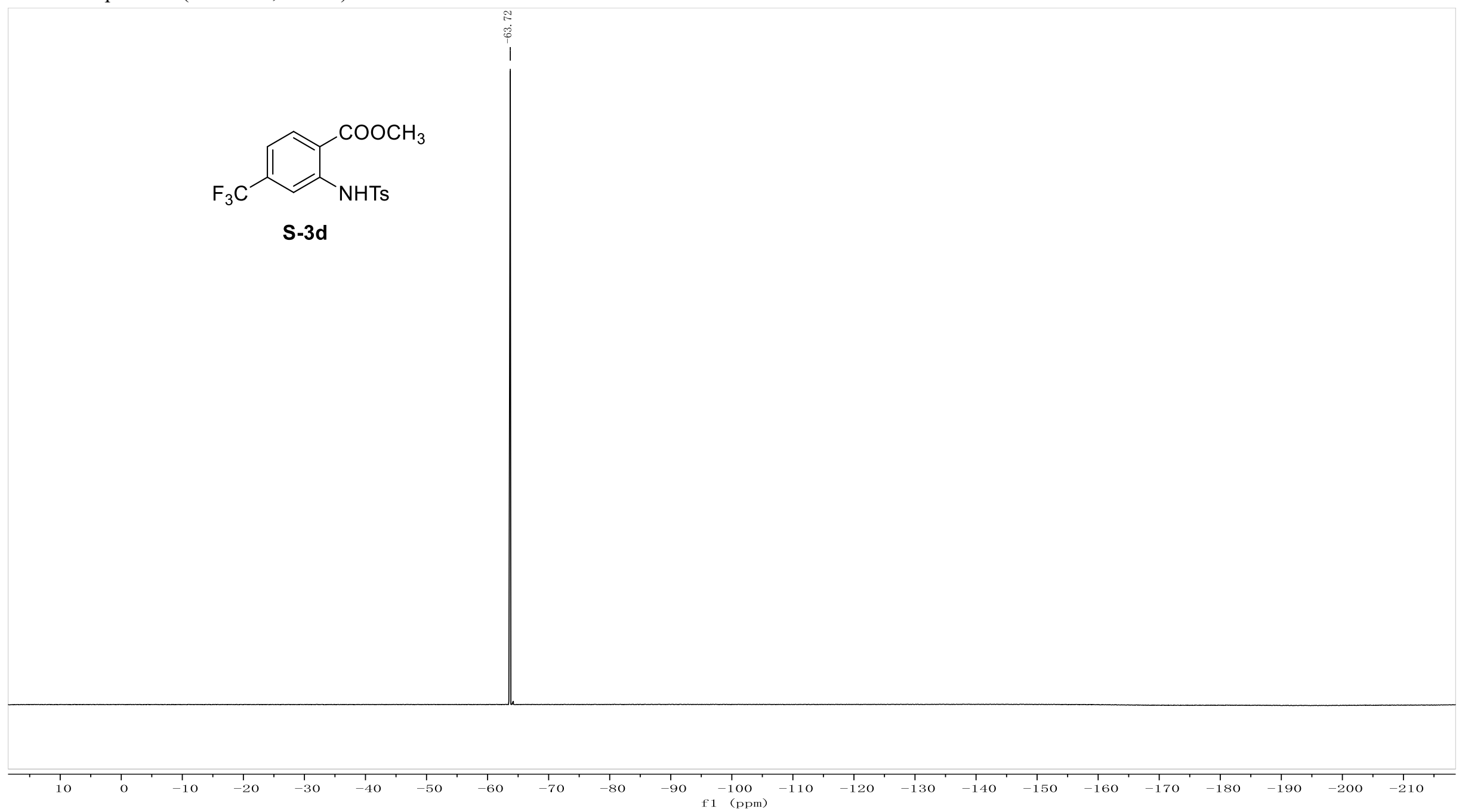


${ }^{1} \mathrm{H}$ NMR-spectrum (400 MHz, DMSO- $\left.d_{6}\right)$ of $\mathbf{S - 4 c}$

要

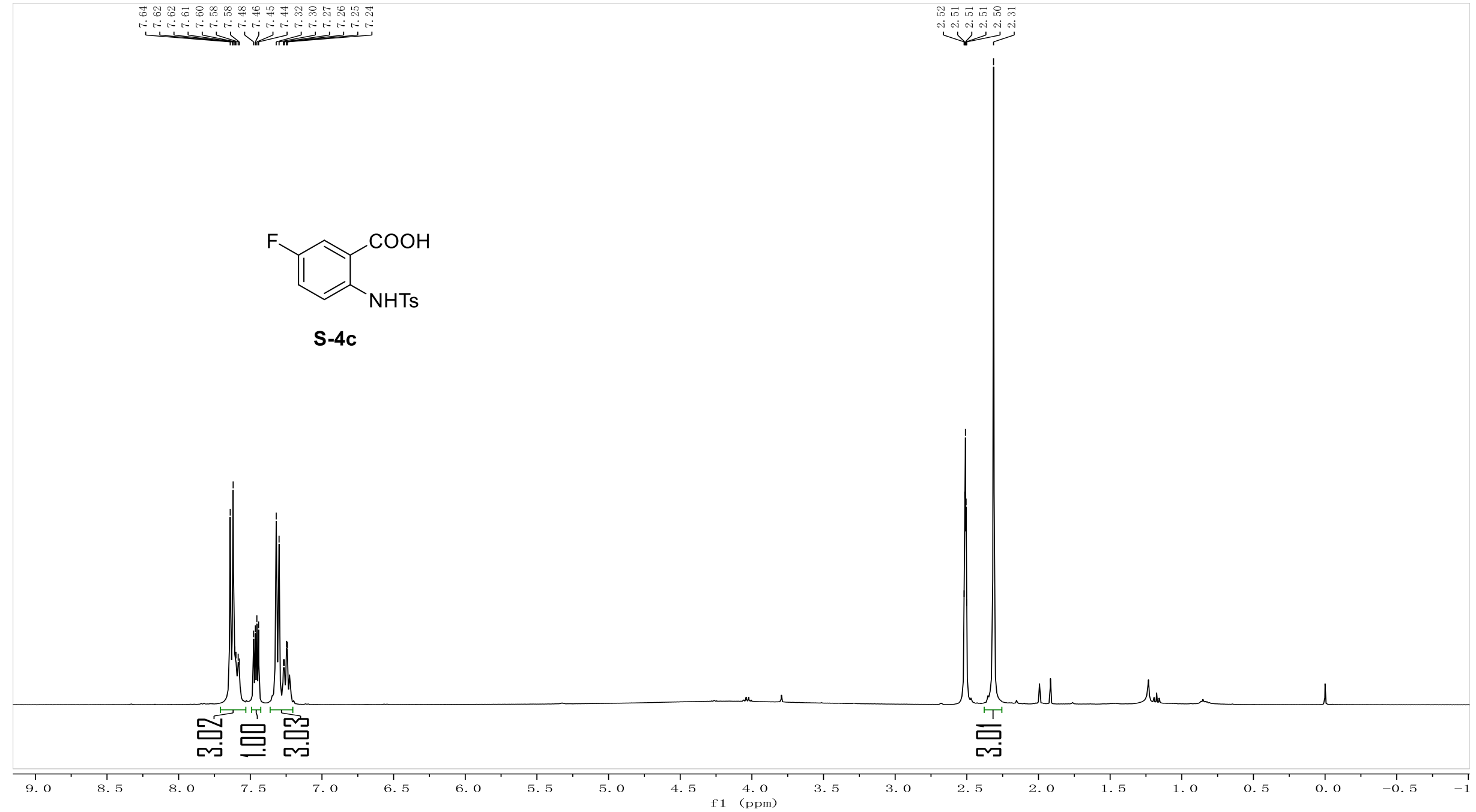




$$
\pm 1
$$


${ }^{19}$ F NMR-spectrum (471 MHz, DMSO- $\left.d_{6}\right)$ of $\mathbf{S}-\mathbf{4 c}$

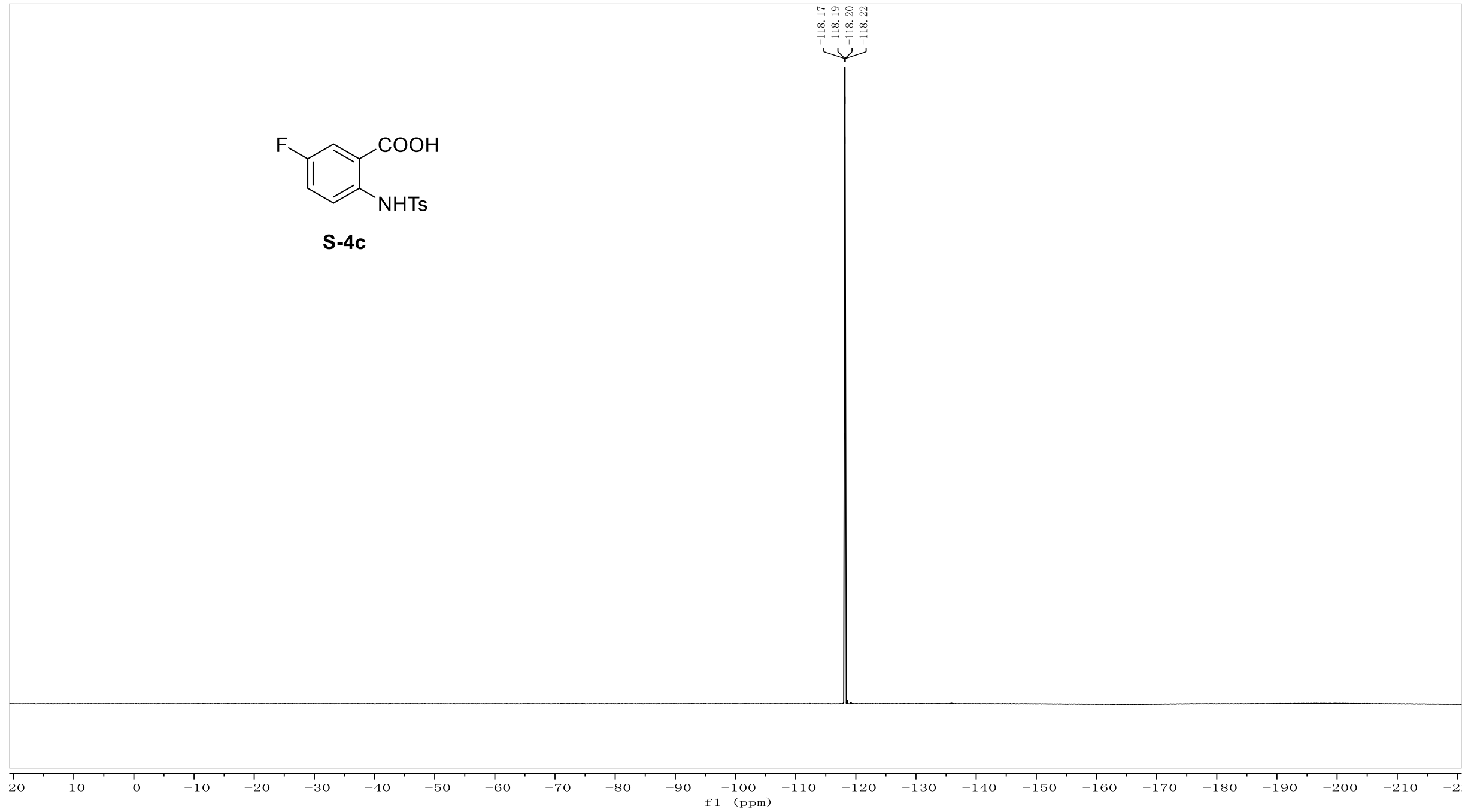


${ }^{1} \mathrm{H}$ NMR-spectrum (400 MHz, DMSO- $\left.d_{6}\right)$ of S-4d

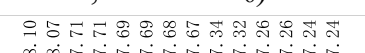
$\underbrace{\infty}$
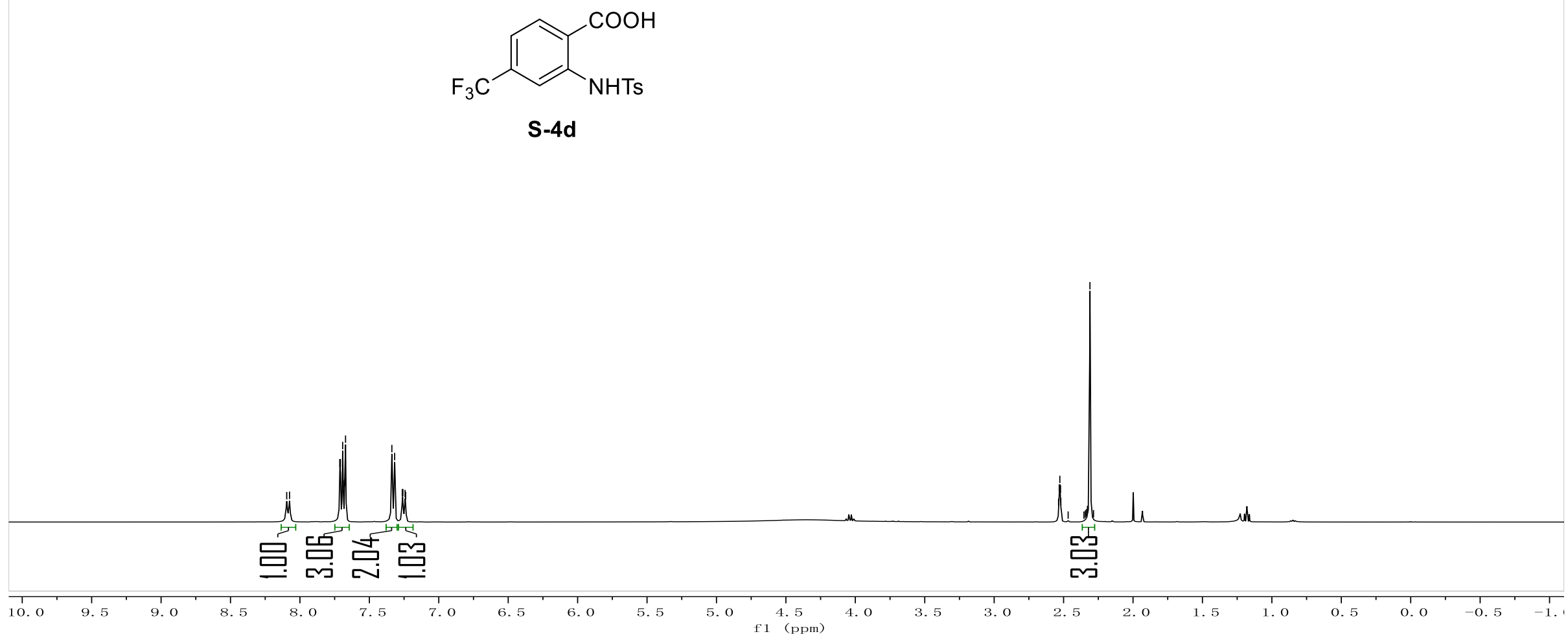
${ }^{13} \mathrm{C}$ NMR-spectrum (101 MHz, DMSO- $\left.d_{6}\right)$ of S-4d

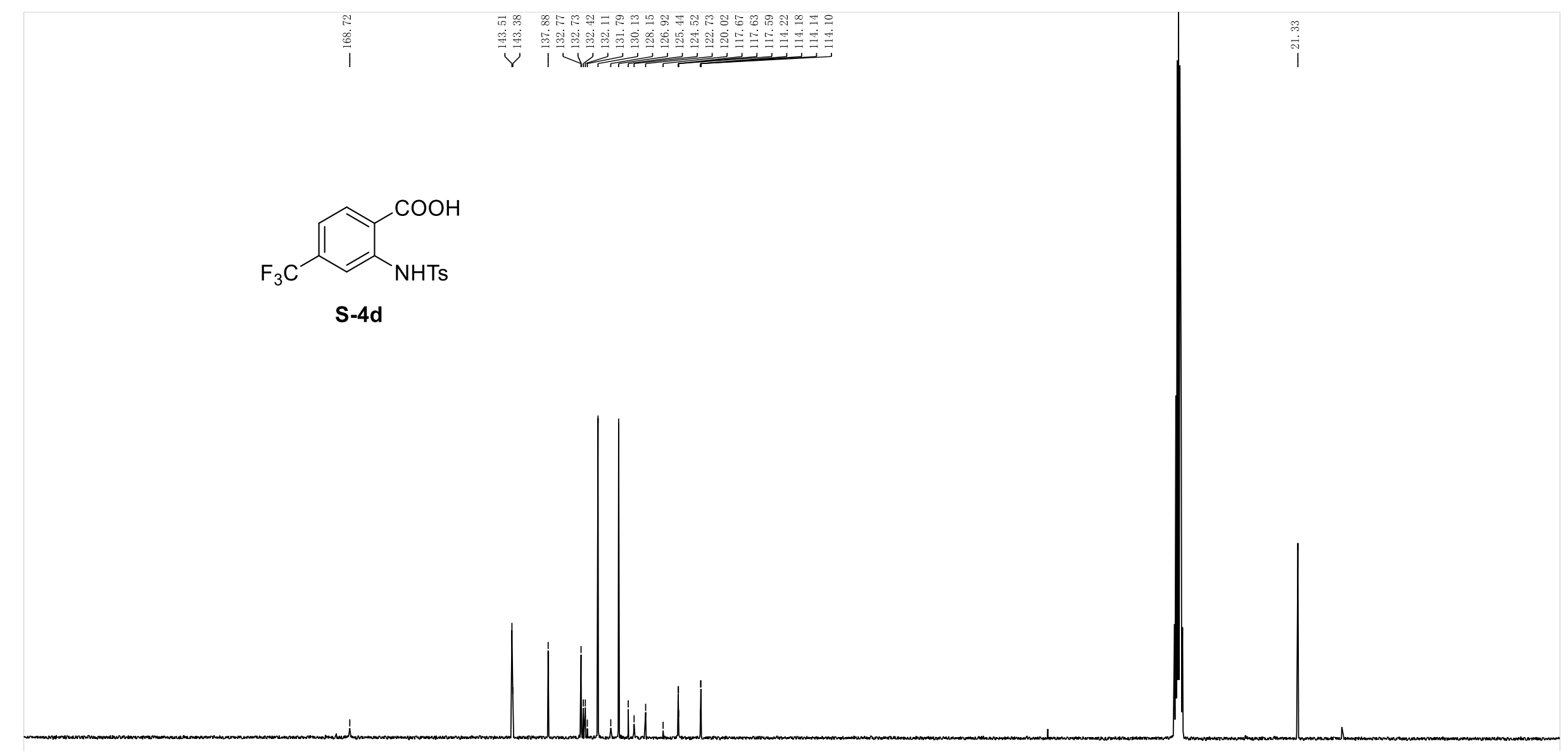

$90 \quad 80 \quad 70 \quad 10$

$50 \quad 40$

$30 \quad 20$ 
${ }^{19}$ F NMR-spectrum (376 MHz, DMSO- $d_{6}$ ) of S-4d

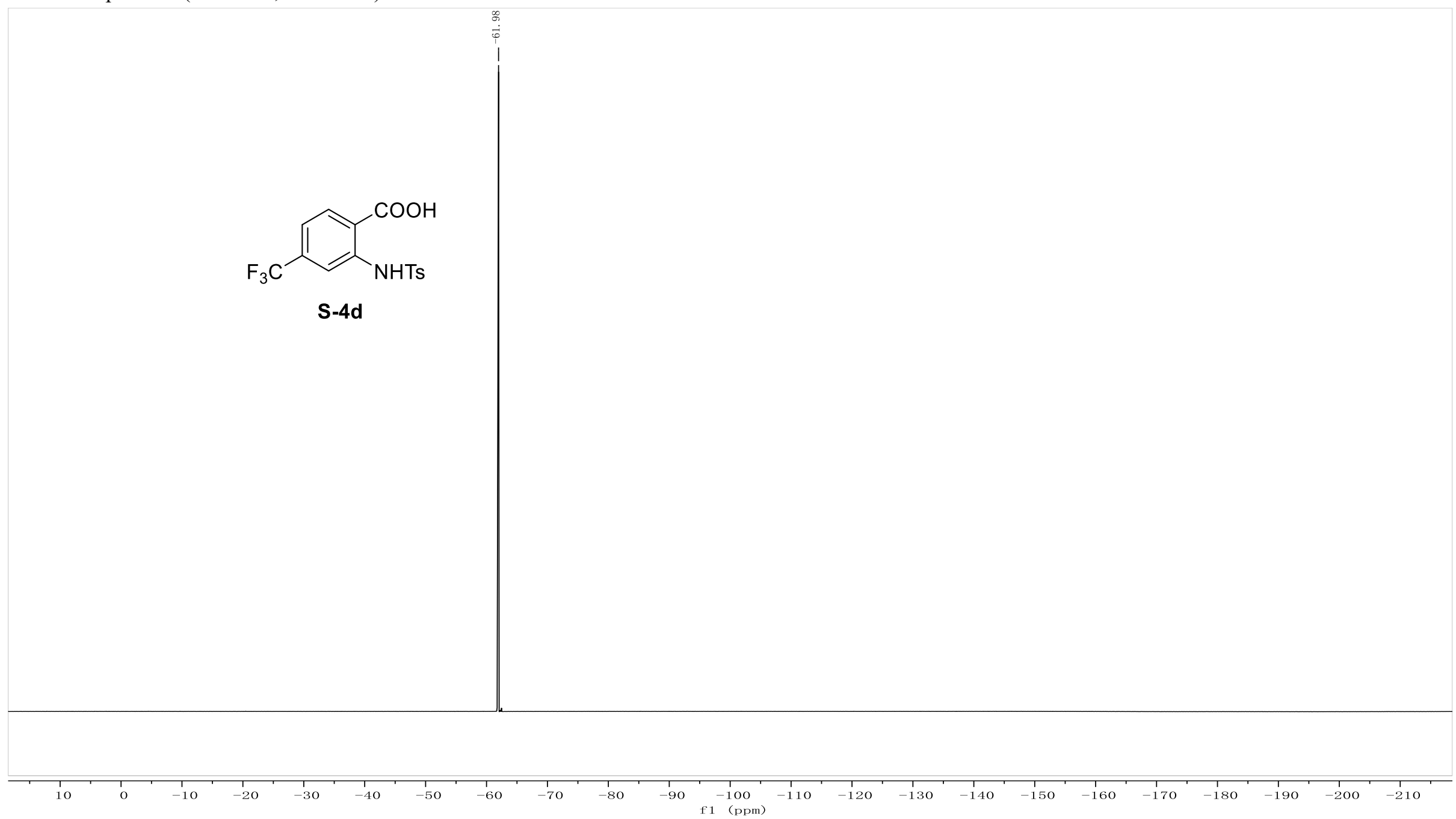


${ }^{1} \mathrm{H}$ NMR-spectrum (500 MHz, DMSO- $d_{6}$ ) of $\mathbf{S - 5}$

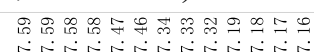

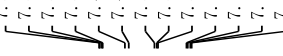

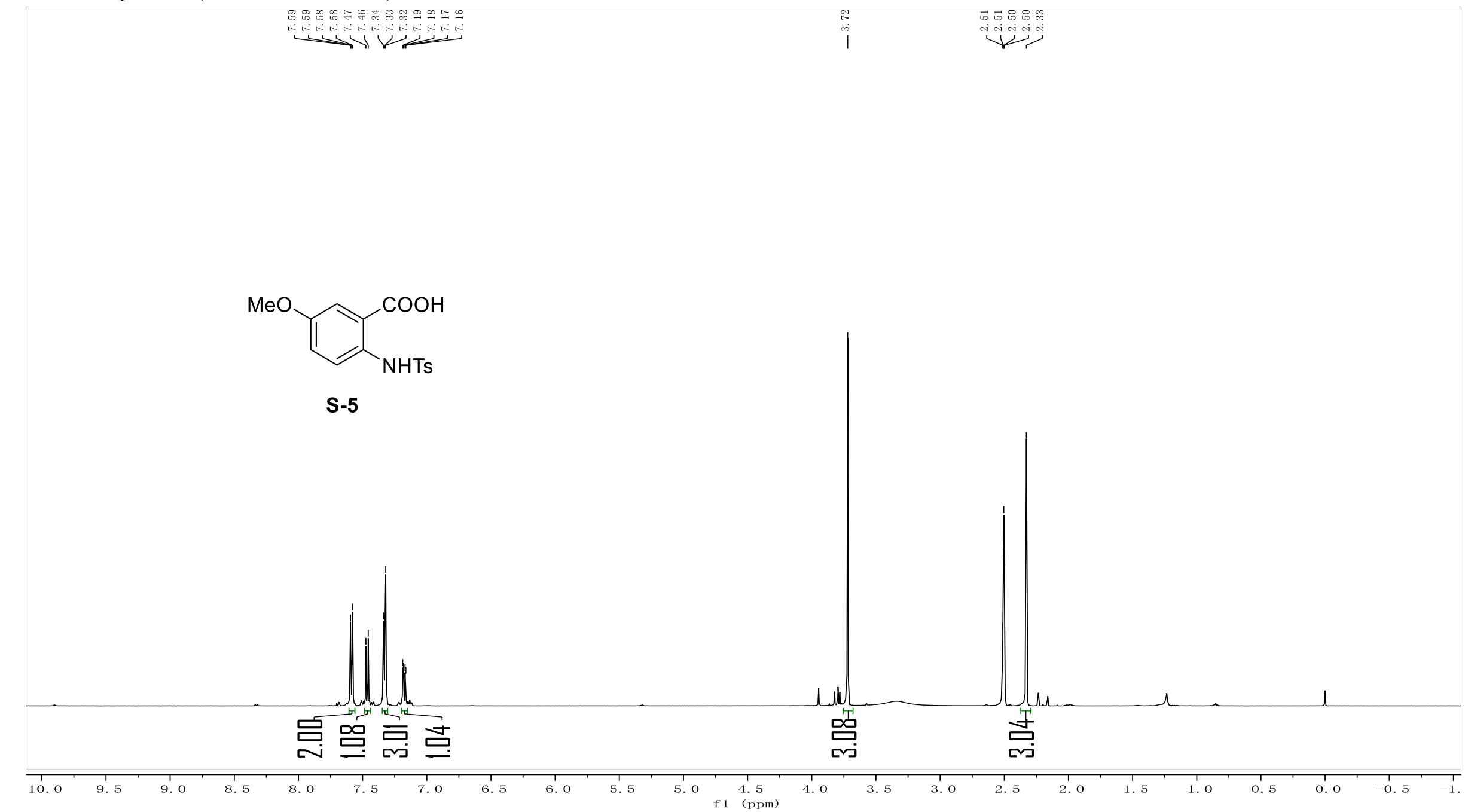


${ }^{13} \mathrm{C}$ NMR-spectrum $\left(126 \mathrm{MHz}, \mathrm{DMSO}-d_{6}\right)$ of $\mathbf{S - 5}$

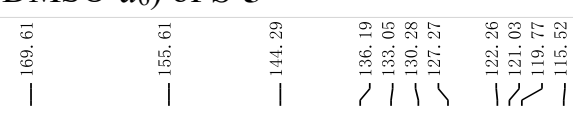

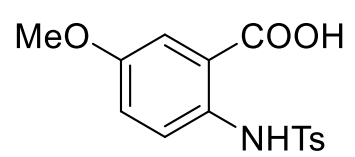

S-5

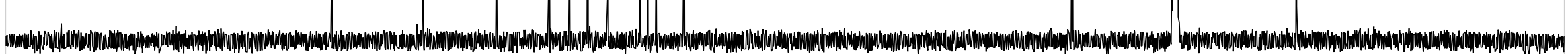
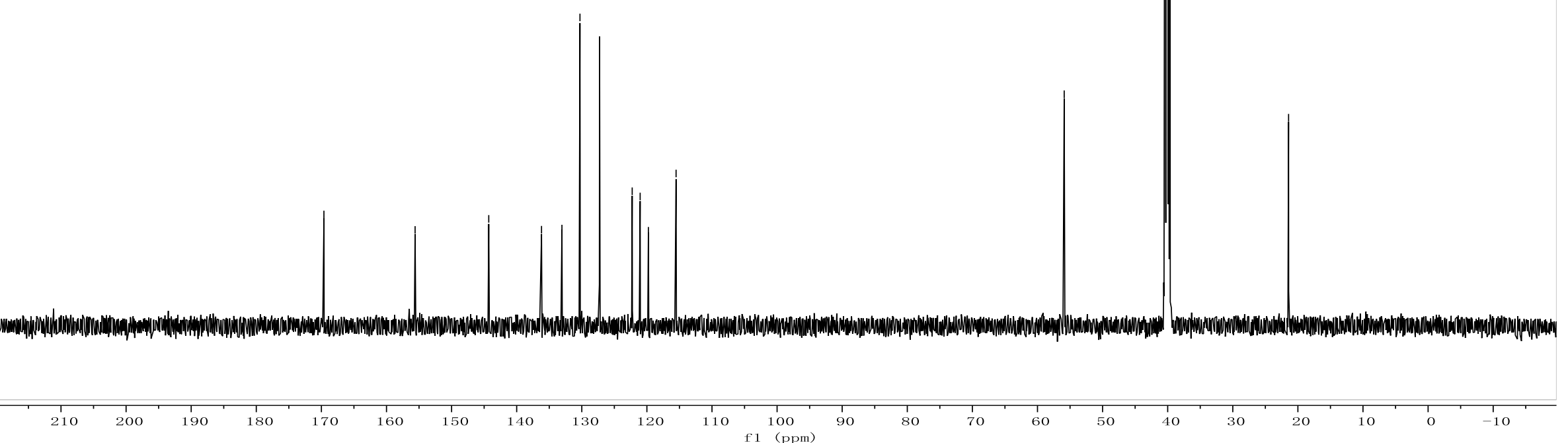
${ }^{1} \mathrm{H}$ NMR-spectrum $\left(400 \mathrm{MHz}, \mathrm{CDCl}_{3}\right)$ of $\mathbf{4 a}$

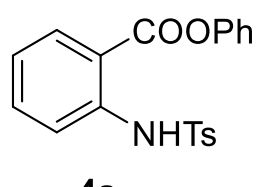

$4 a$

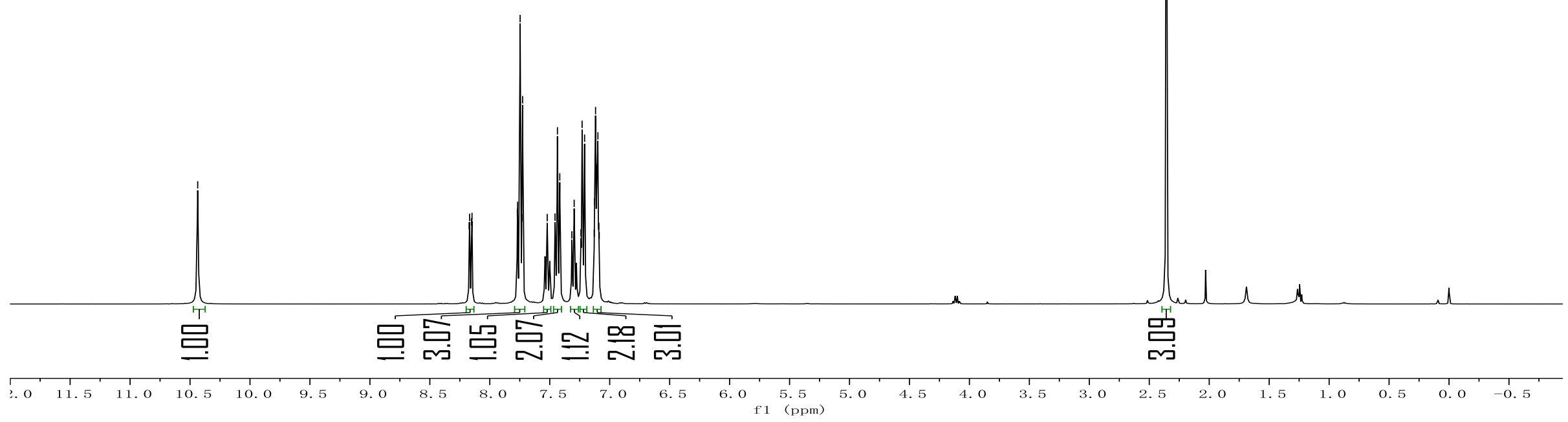


${ }^{13} \mathrm{C}$ NMR-spectrum $\left(101 \mathrm{MHz}, \mathrm{CDCl}_{3}\right)$ of $\mathbf{4 a}$
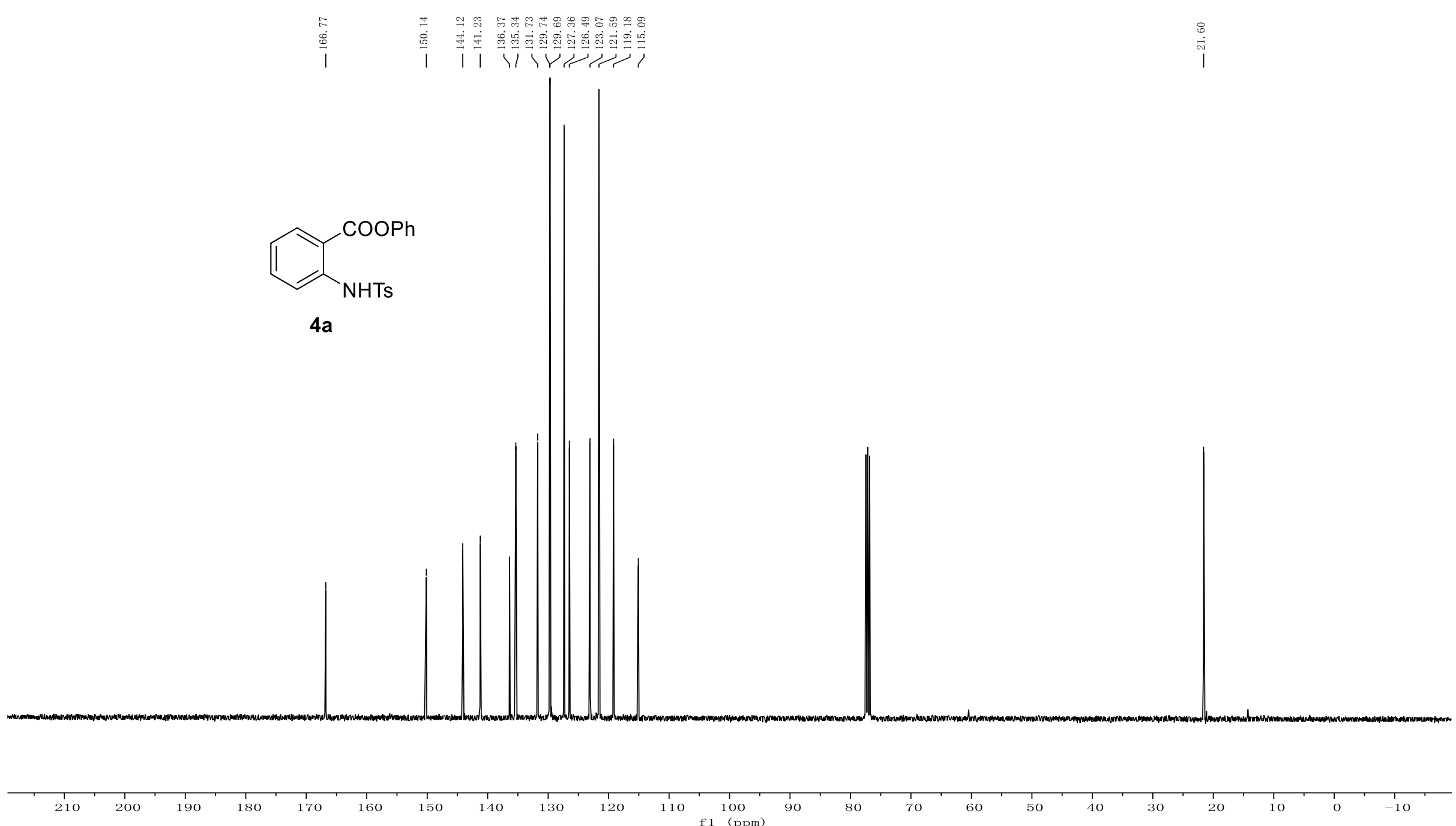
${ }^{1} \mathrm{H}$ NMR-spectrum $\left(400 \mathrm{MHz}, \mathrm{CDCl}_{3}\right)$ of $\mathbf{4 c}$

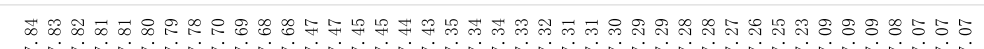

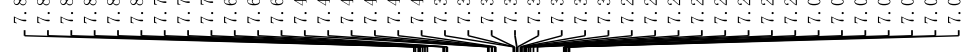

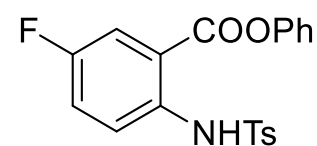

4c

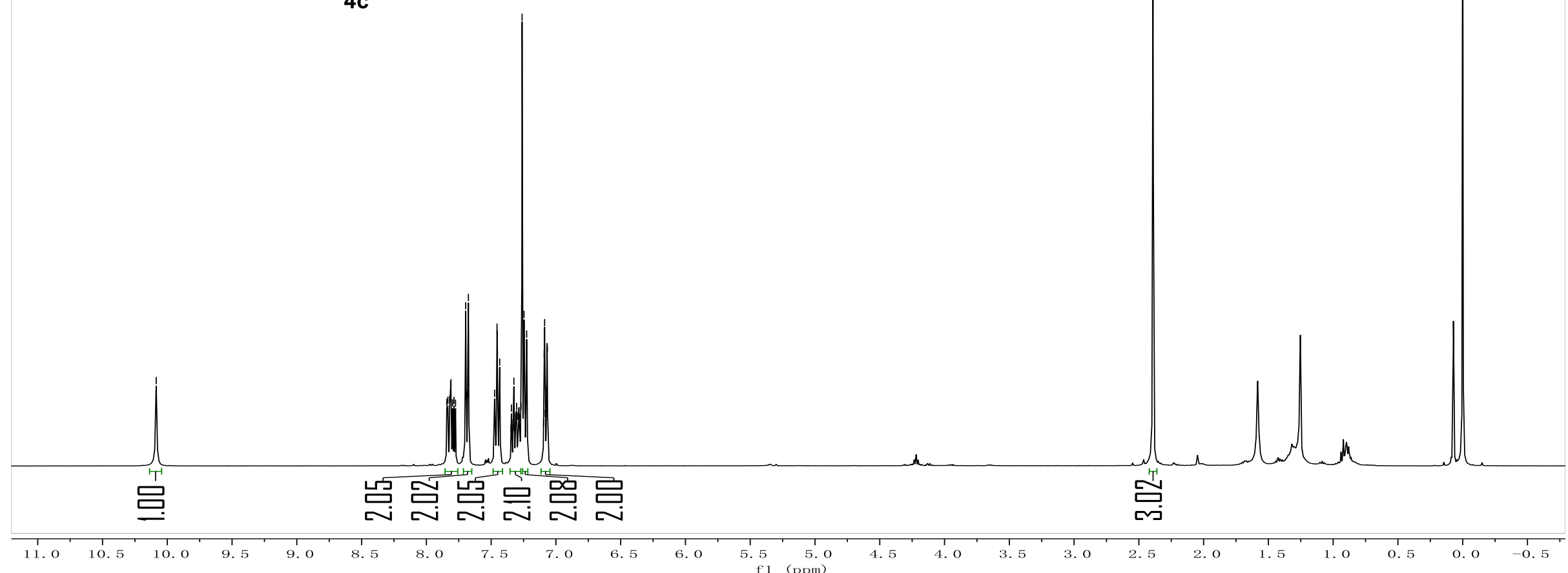


${ }^{13} \mathrm{C}$ NMR-spectrum $\left(101 \mathrm{MHz}, \mathrm{CDCl}_{3}\right)$ of $\mathbf{4 c}$

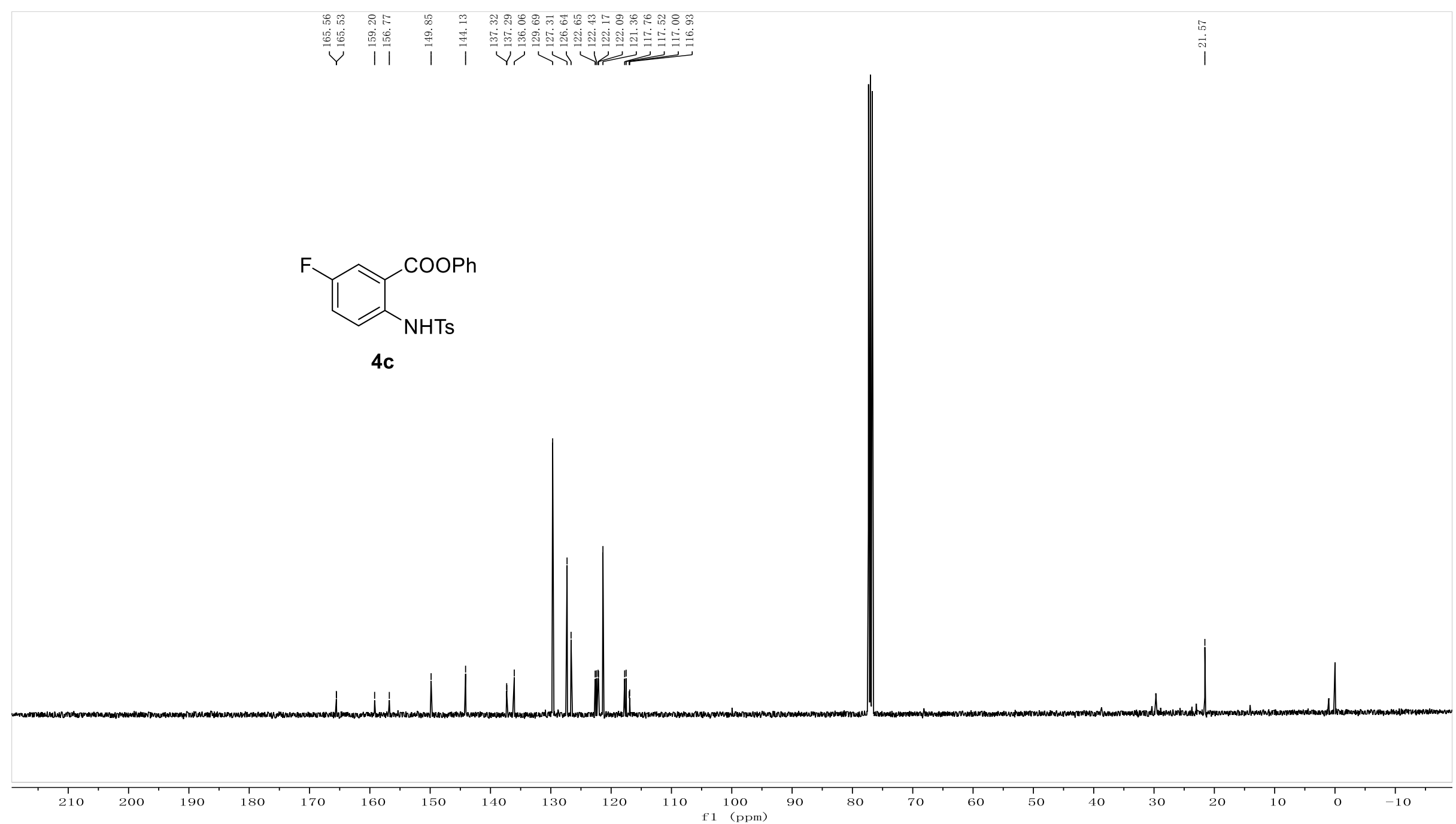


${ }^{19} \mathrm{~F}$ NMR-spectrum $\left(376 \mathrm{MHz}, \mathrm{CDCl}_{3}\right)$ of $\mathbf{4 c}$

$\therefore \therefore \infty$

音至今

12

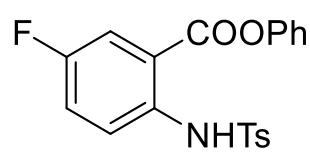

4c

NHTs

\section{㕷}

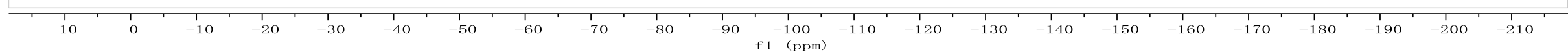


${ }^{1} \mathrm{H}$ NMR-spectrum $\left(400 \mathrm{MHz}, \mathrm{CDCl}_{3}\right)$ of $\mathbf{4 b}$

我

14

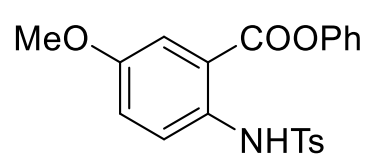

4b
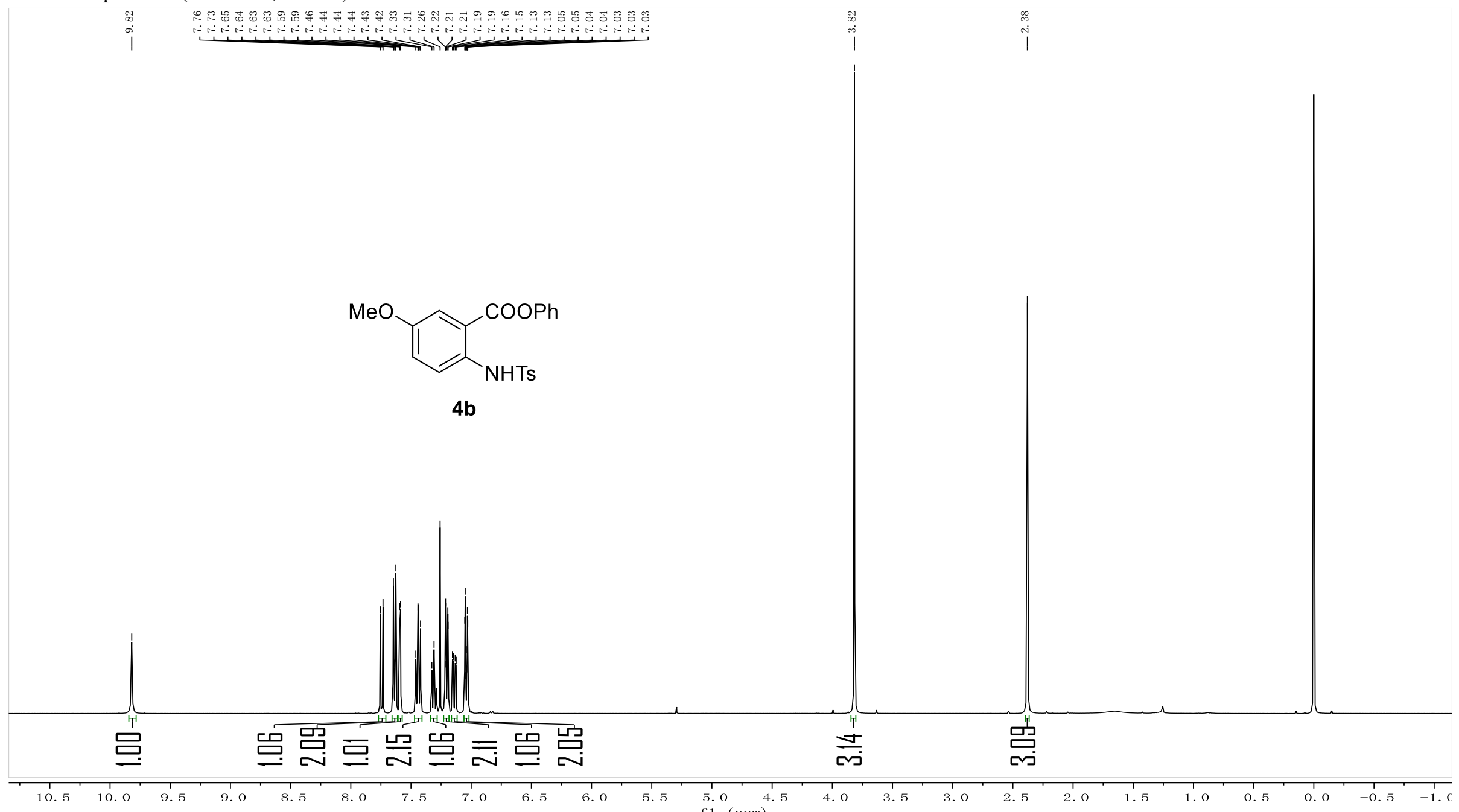
${ }^{13} \mathrm{C}$ NMR-spectrum $\left(101 \mathrm{MHz}, \mathrm{CDCl}_{3}\right)$ of $\mathbf{4 b}$

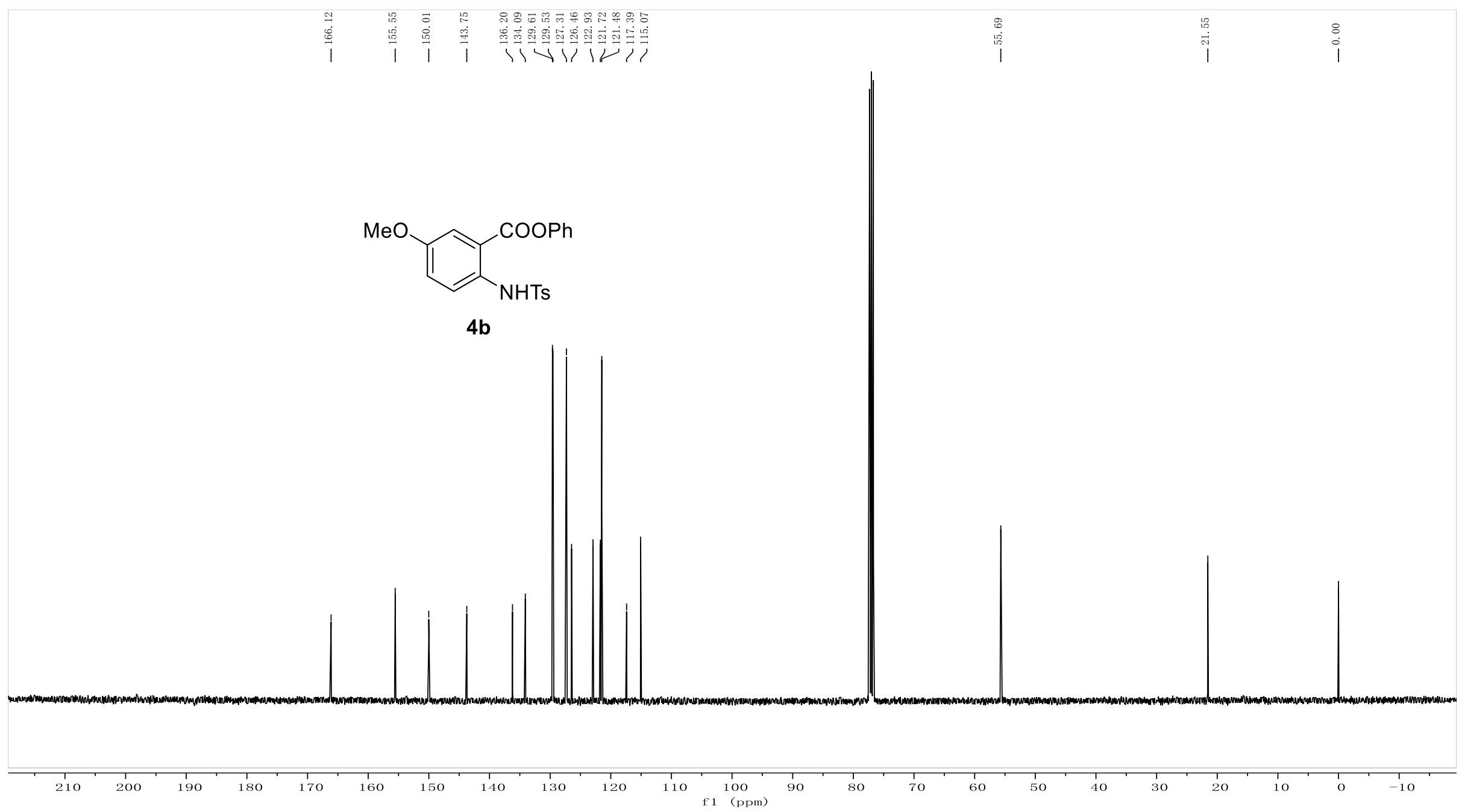

S58 
${ }^{1} \mathrm{H}$ NMR-spectrum $\left(400 \mathrm{MHz}, \mathrm{CDCl}_{3}\right)$ of $\mathbf{4 d}$

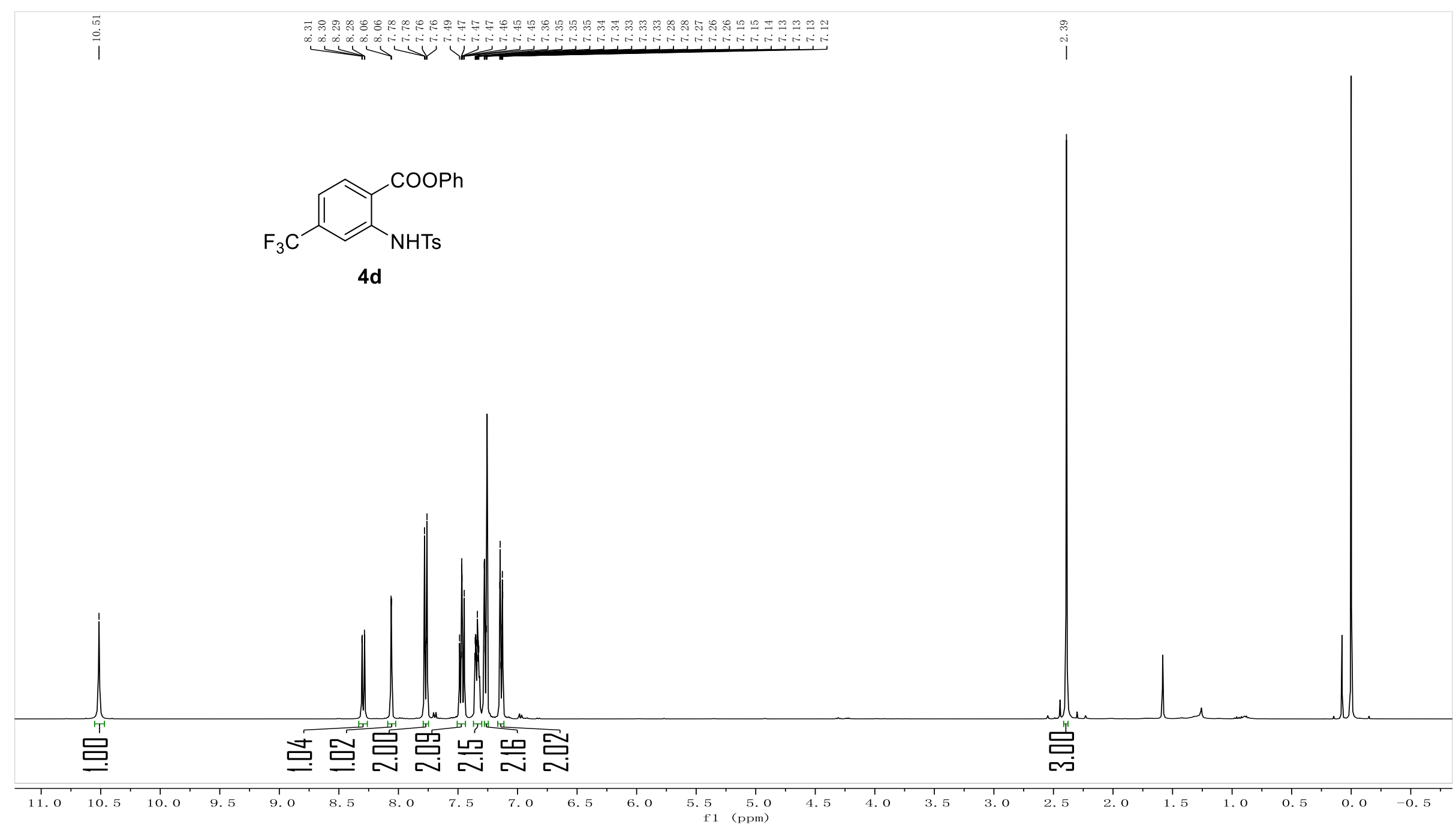


${ }^{13} \mathrm{C}$ NMR-spectrum $\left(101 \mathrm{MHz}, \mathrm{CDCl}_{3}\right)$ of $\mathbf{4 d}$

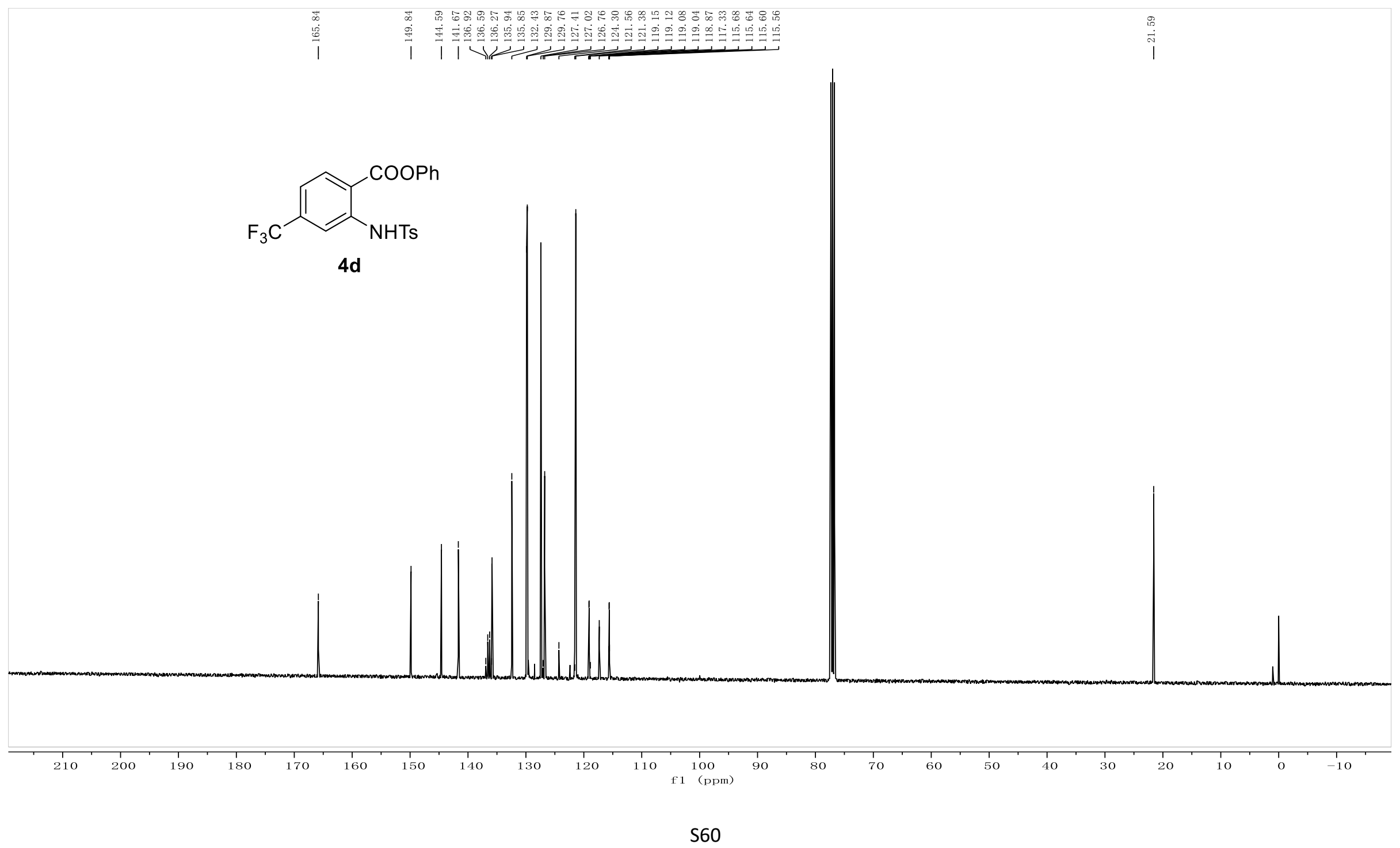


${ }^{19} \mathrm{~F}$ NMR-spectrum $\left(376 \mathrm{MHz}, \mathrm{CDCl}_{3}\right)$ of $\mathbf{4 d}$

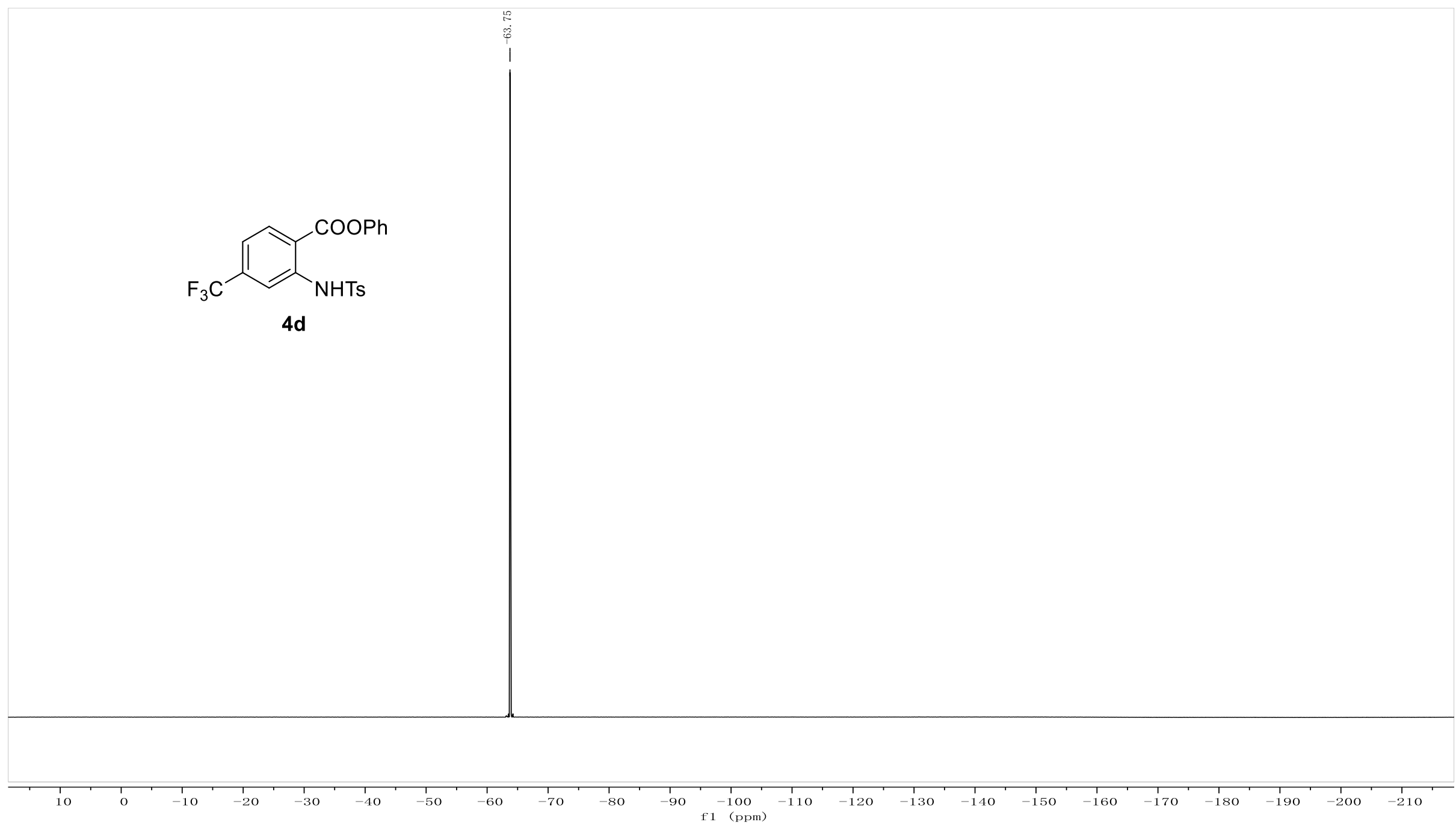


${ }^{1} \mathrm{H}$ NMR-spectrum $\left(500 \mathrm{MHz}, \mathrm{CDCl}_{3}\right)$ of $\mathbf{2 m}$

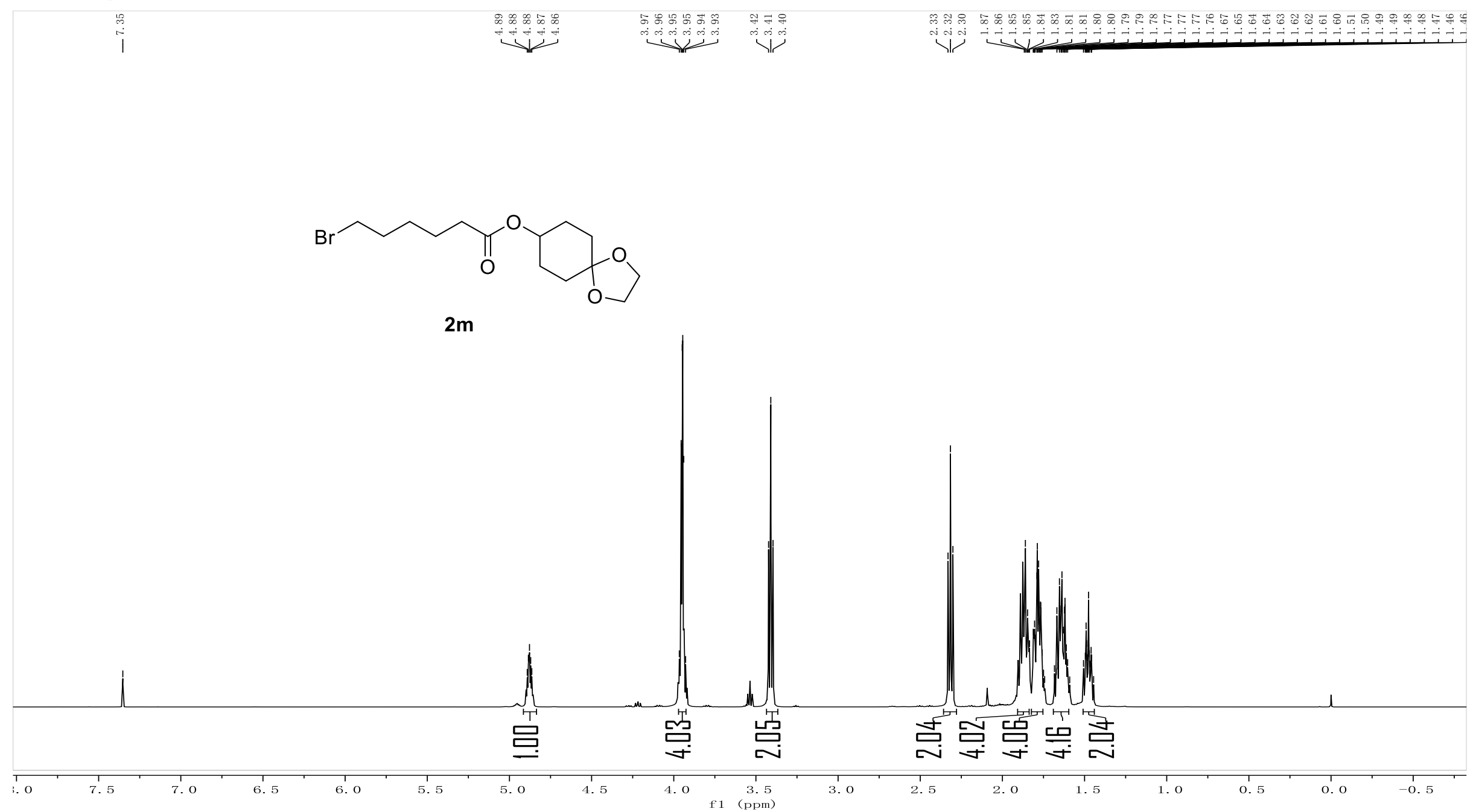


${ }^{13} \mathrm{C}$ NMR-spectrum $\left(126 \mathrm{MHz}, \mathrm{CDCl}_{3}\right)$ of $\mathbf{2 m}$
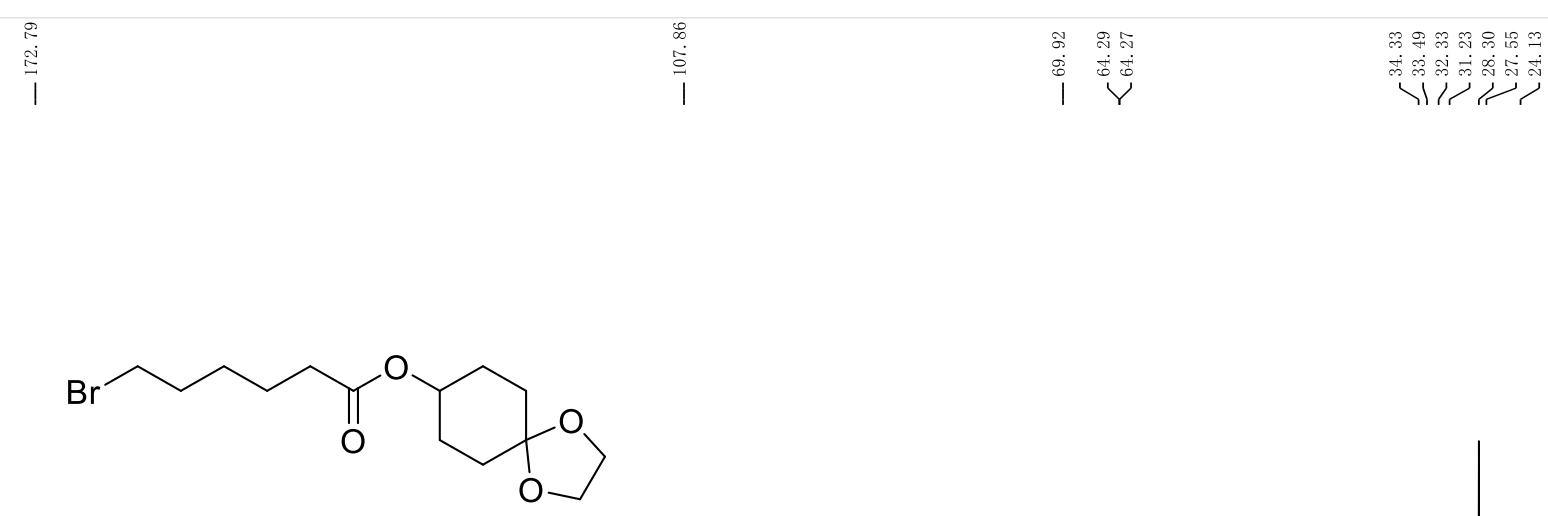

$2 m$

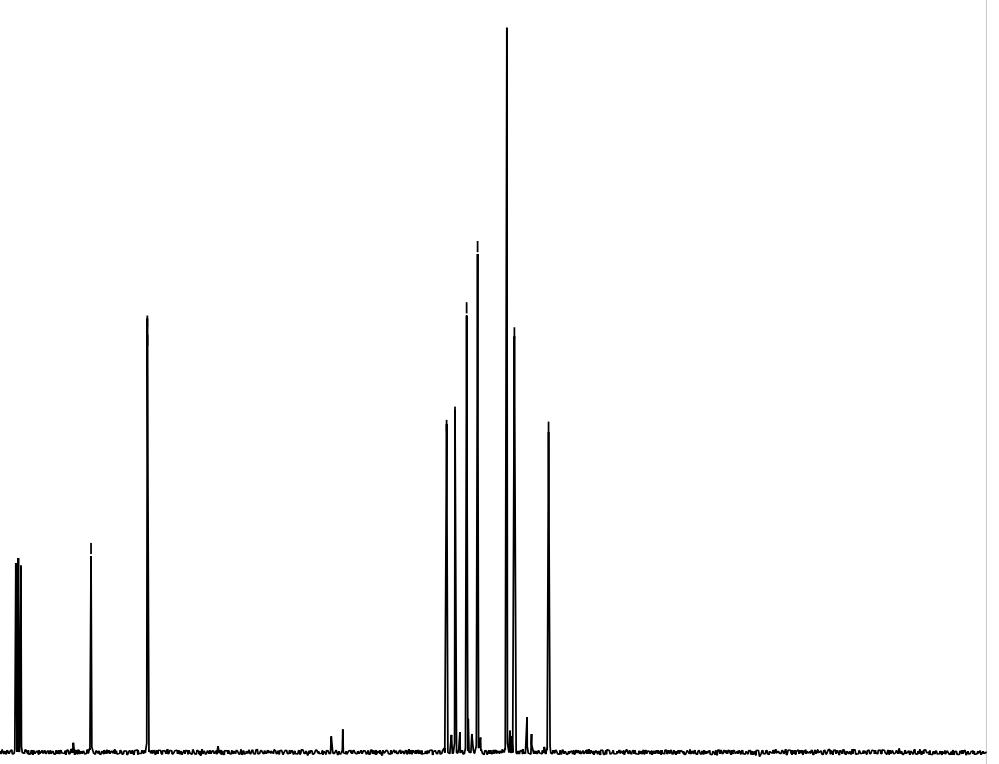


${ }^{1} \mathrm{H}$ NMR-spectrum $\left(500 \mathrm{MHz}, \mathrm{CDCl}_{3}\right)$ of $\mathbf{2 r}$

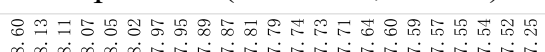
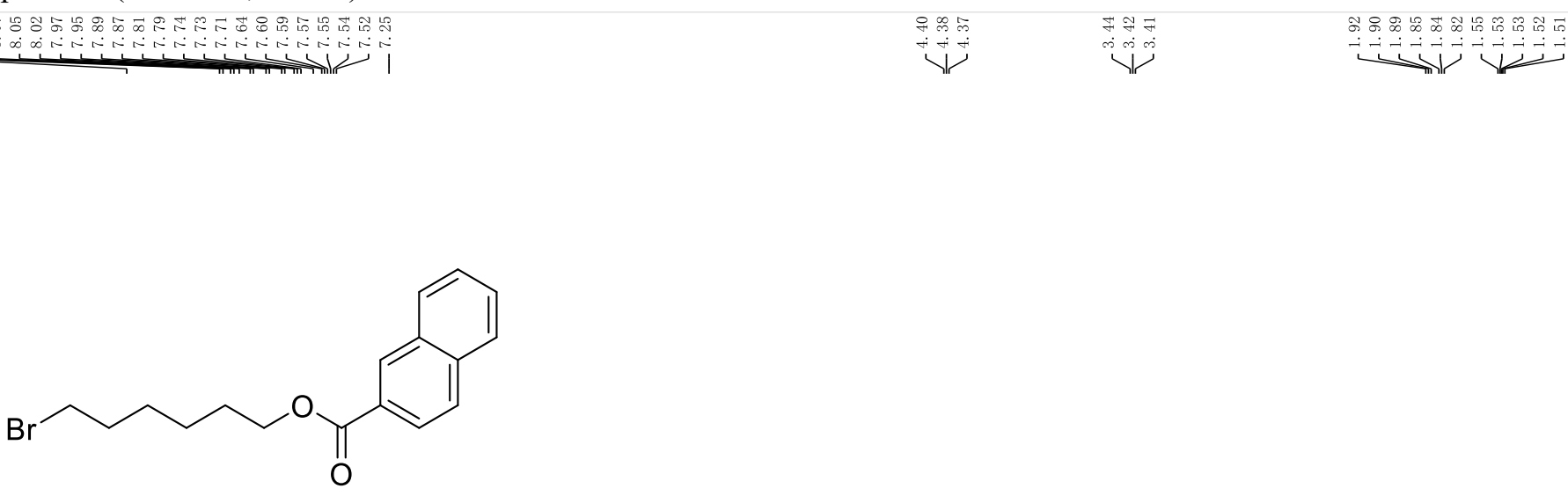

$2 r$

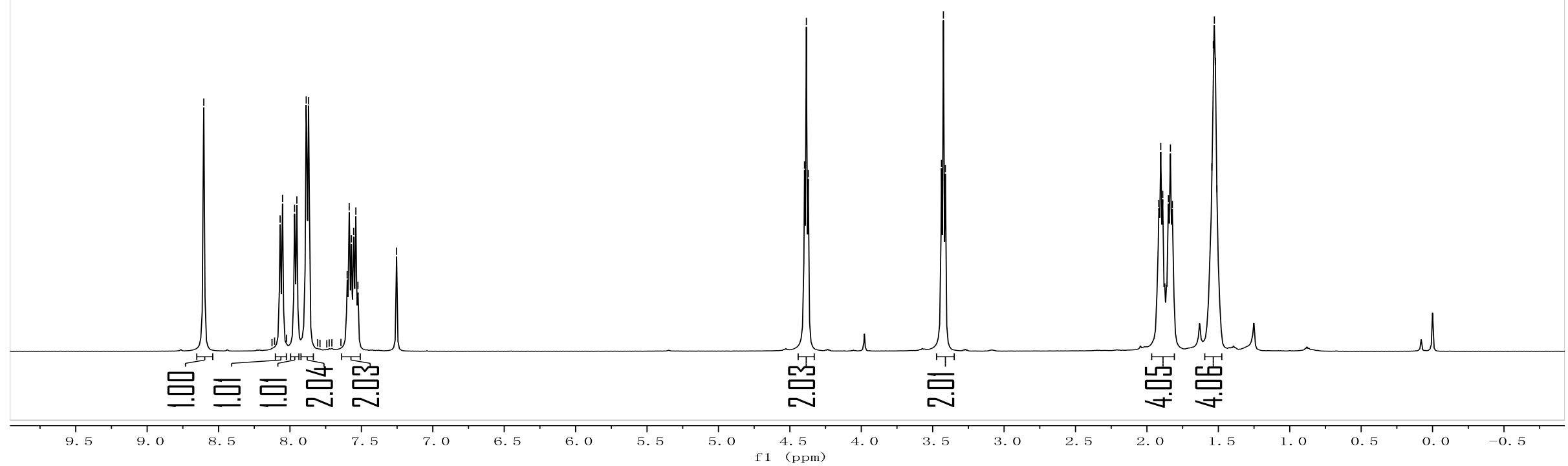


${ }^{13} \mathrm{C}$ NMR-spectrum $\left(101 \mathrm{MHz}, \mathrm{CDCl}_{3}\right)$ of $\mathbf{2 r}$
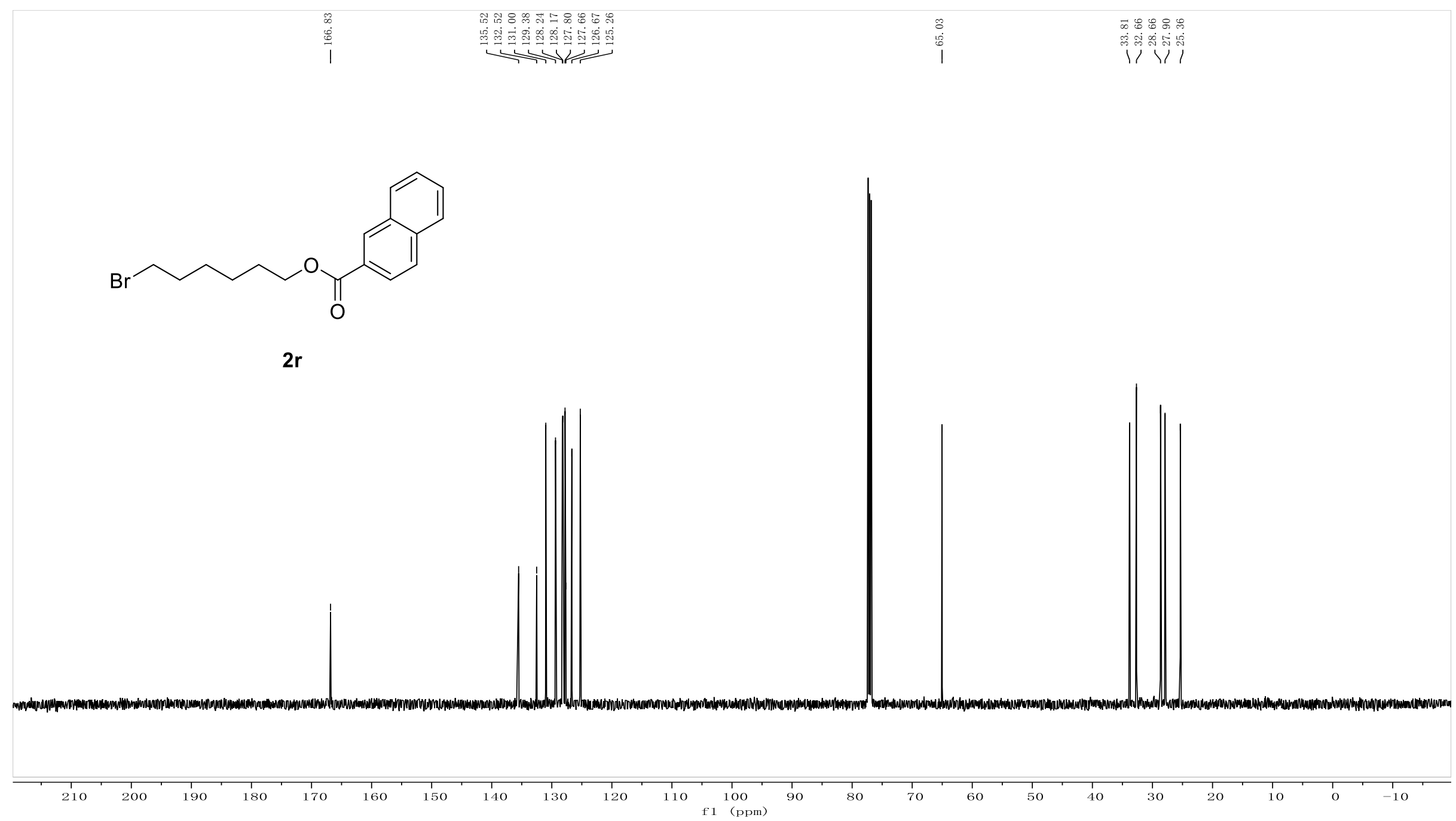
${ }^{1} \mathrm{H}$ NMR-spectrum $\left(400 \mathrm{MHz}, \mathrm{CDCl}_{3}\right)$ of $\mathbf{3 a a}$

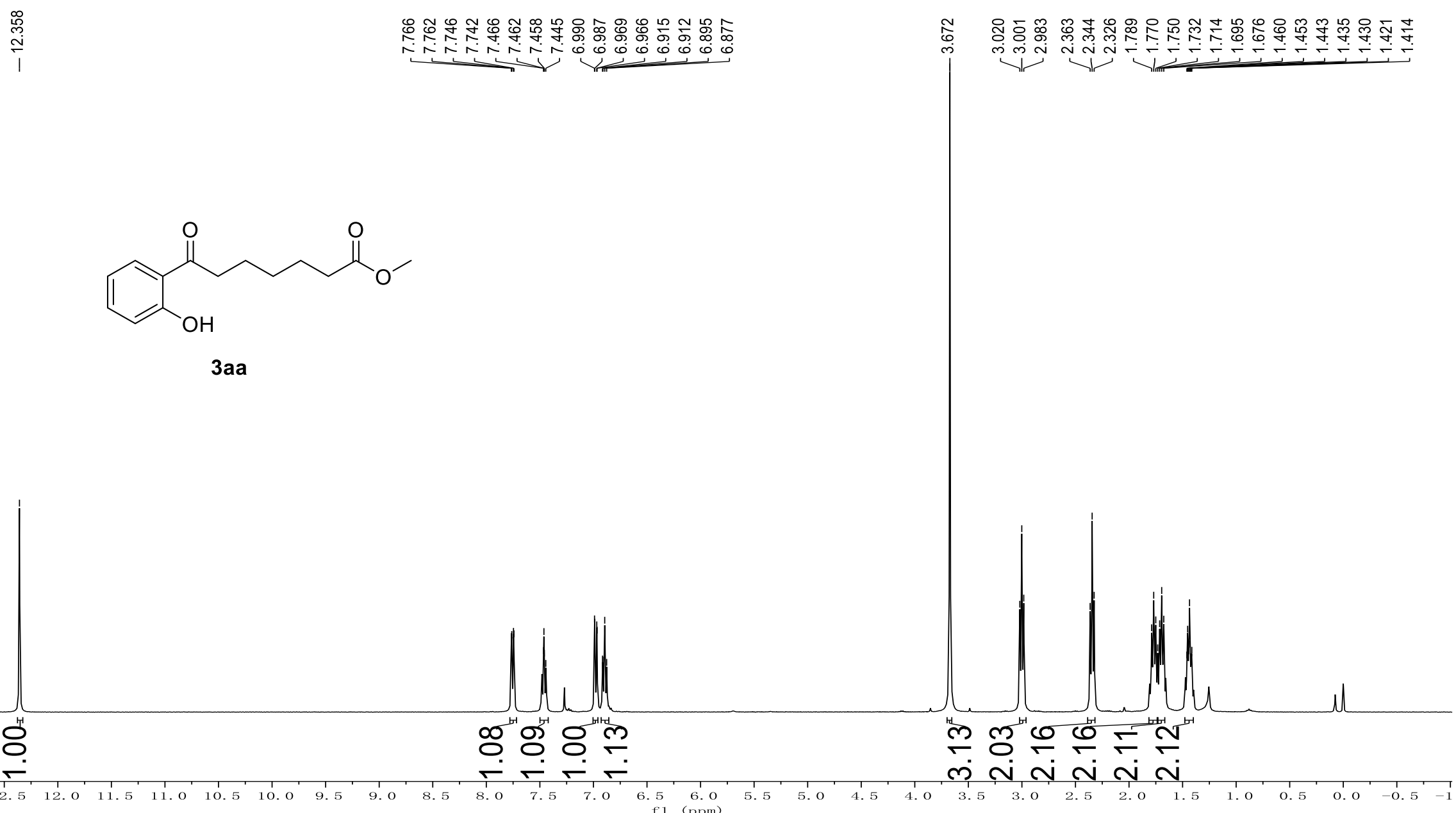


${ }^{13} \mathrm{C}$ NMR-spectrum $\left(101 \mathrm{MHz}, \mathrm{CDCl}_{3}\right)$ of $\mathbf{3 a a}$
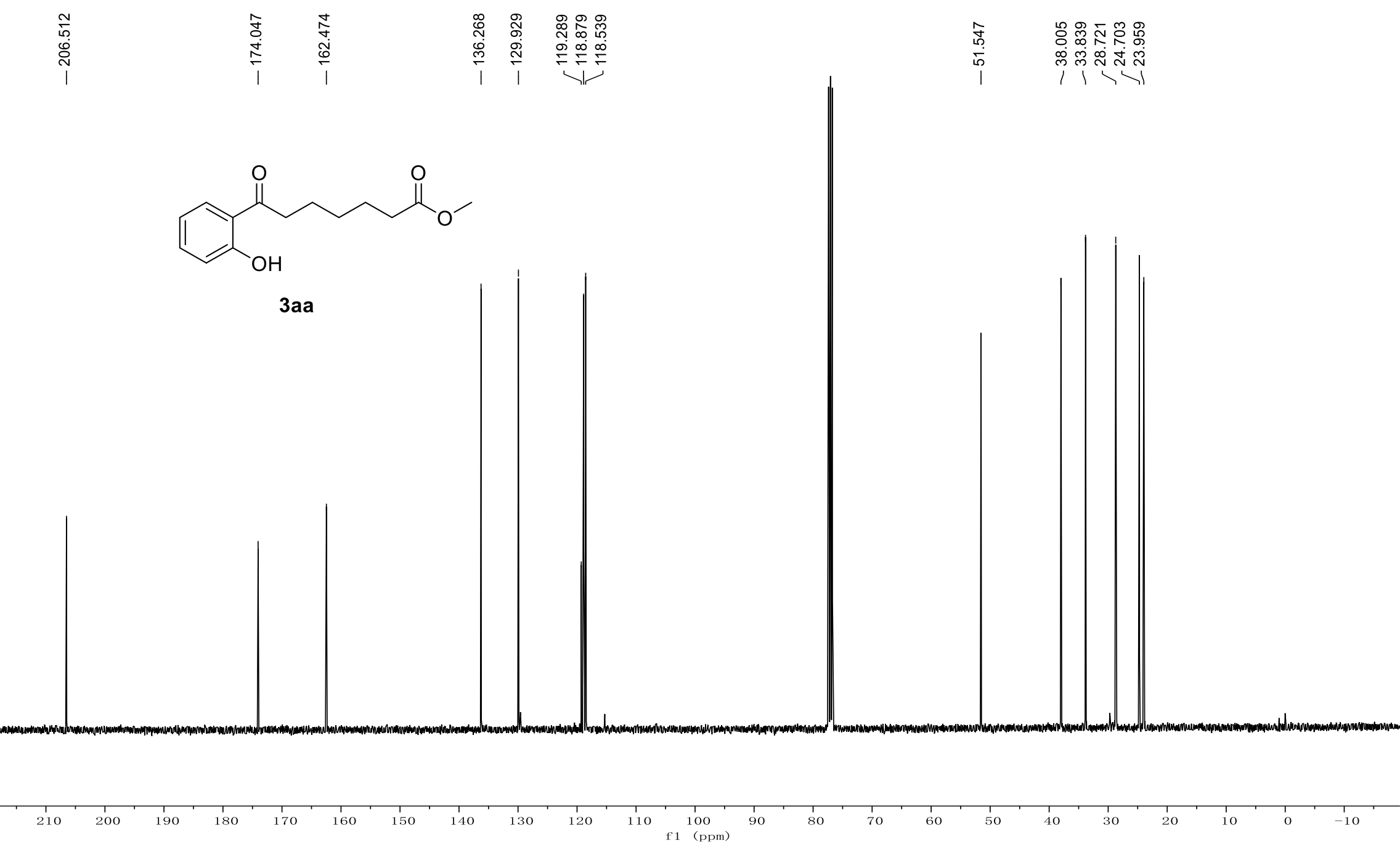
${ }^{1} \mathrm{H}$ NMR-spectrum $\left(500 \mathrm{MHz}, \mathrm{CDCl}_{3}\right)$ of $\mathbf{3 a b}$

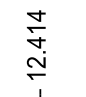

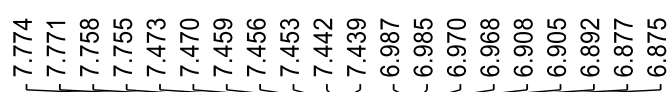

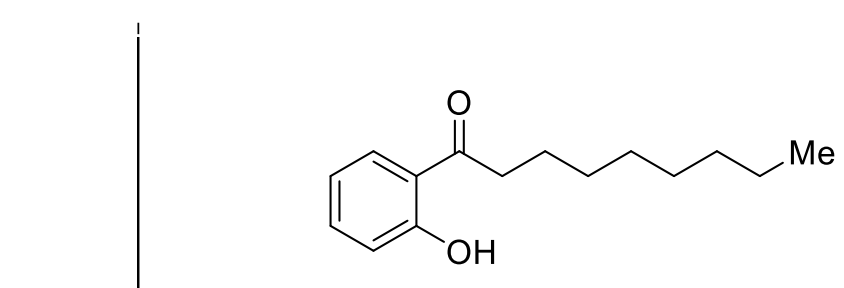

$3 a b$
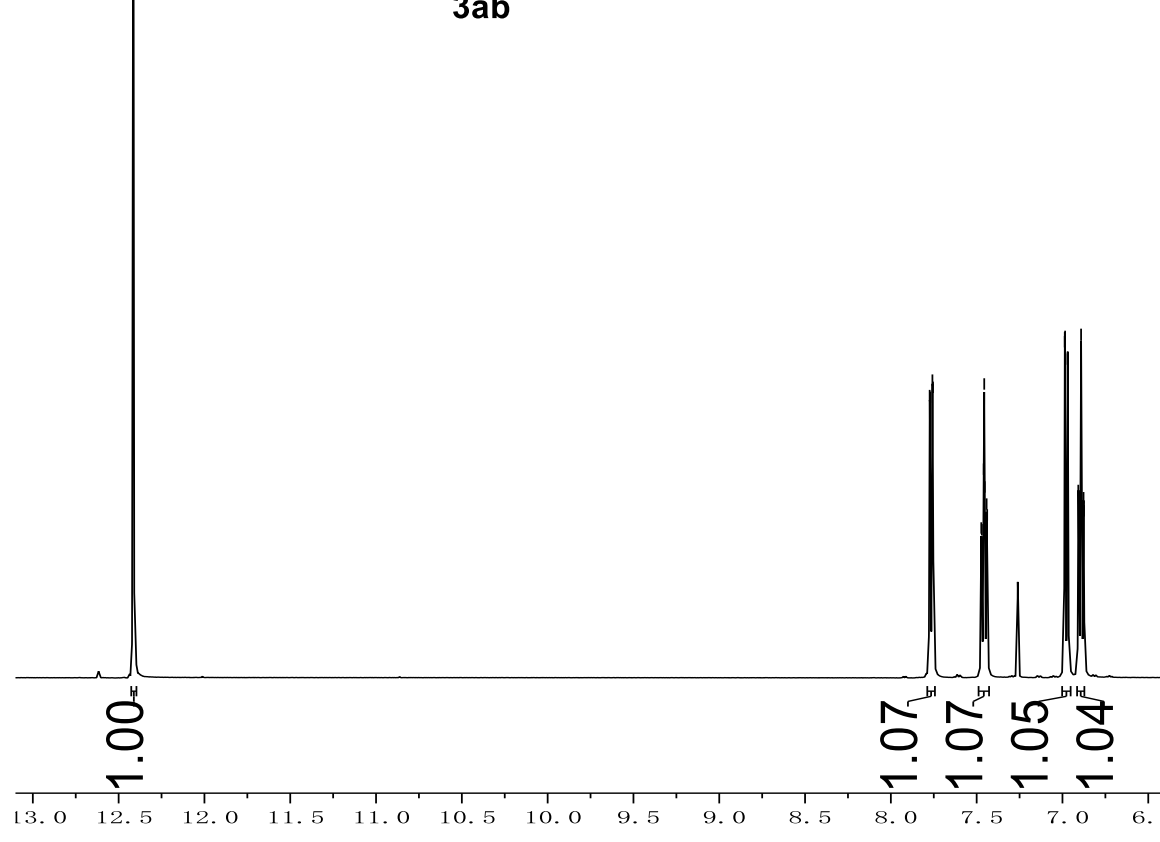

$\begin{array}{ll}5 & 6.0 \\ \mathrm{f} 1 & (\mathrm{ppm})\end{array}$ 
${ }^{13} \mathrm{C}$ NMR-spectrum $\left(126 \mathrm{MHz}, \mathrm{CDCl}_{3}\right)$ of $\mathbf{3 a b}$

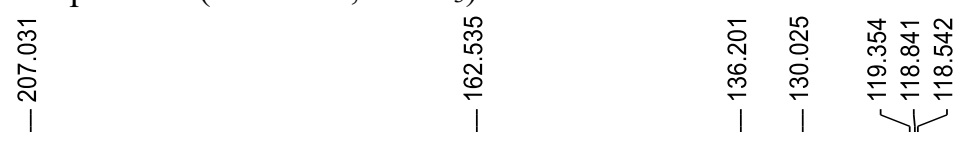

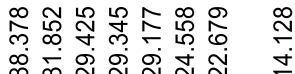

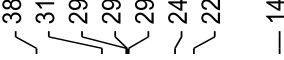

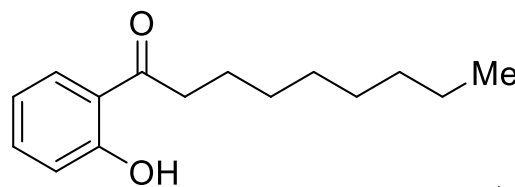

$3 a b$

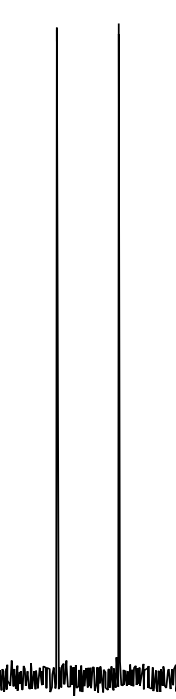

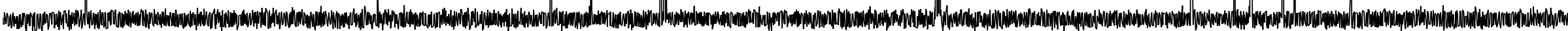
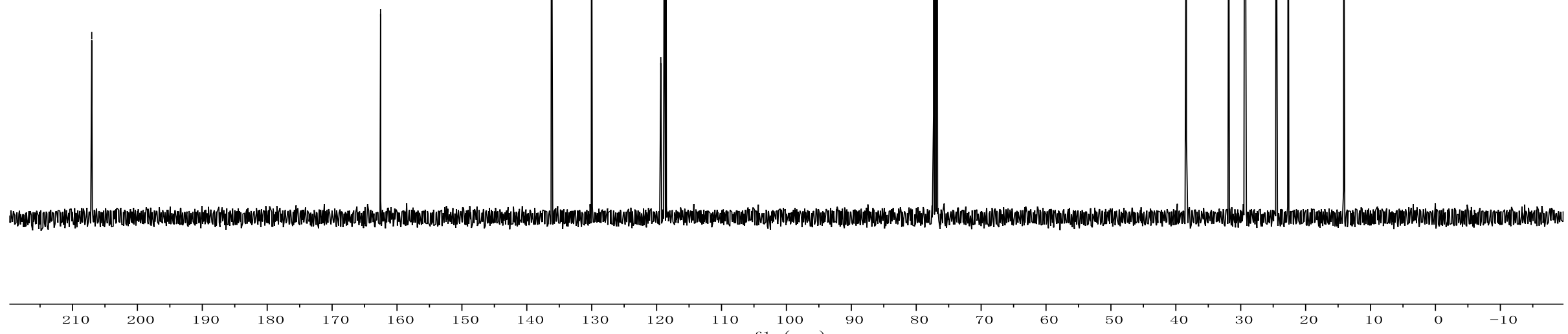
${ }^{1} \mathrm{H}$ NMR-spectrum $\left(500 \mathrm{MHz}, \mathrm{CDCl}_{3}\right)$ of $\mathbf{3 a c}$

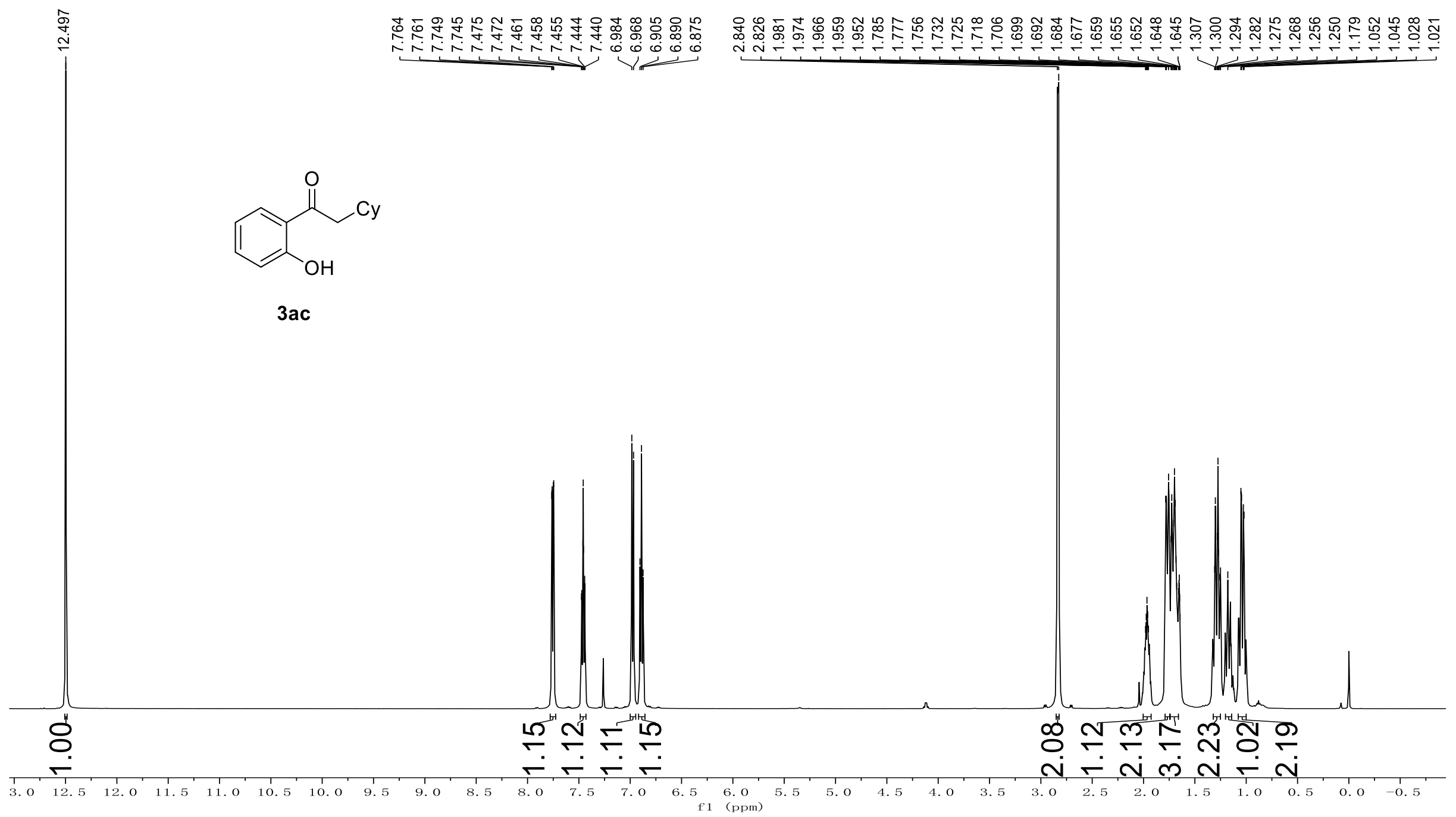


${ }^{13} \mathrm{C}$ NMR-spectrum $\left(126 \mathrm{MHz}, \mathrm{CDCl}_{3}\right)$ of $\mathbf{3 a c}$

品

i

।

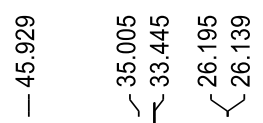
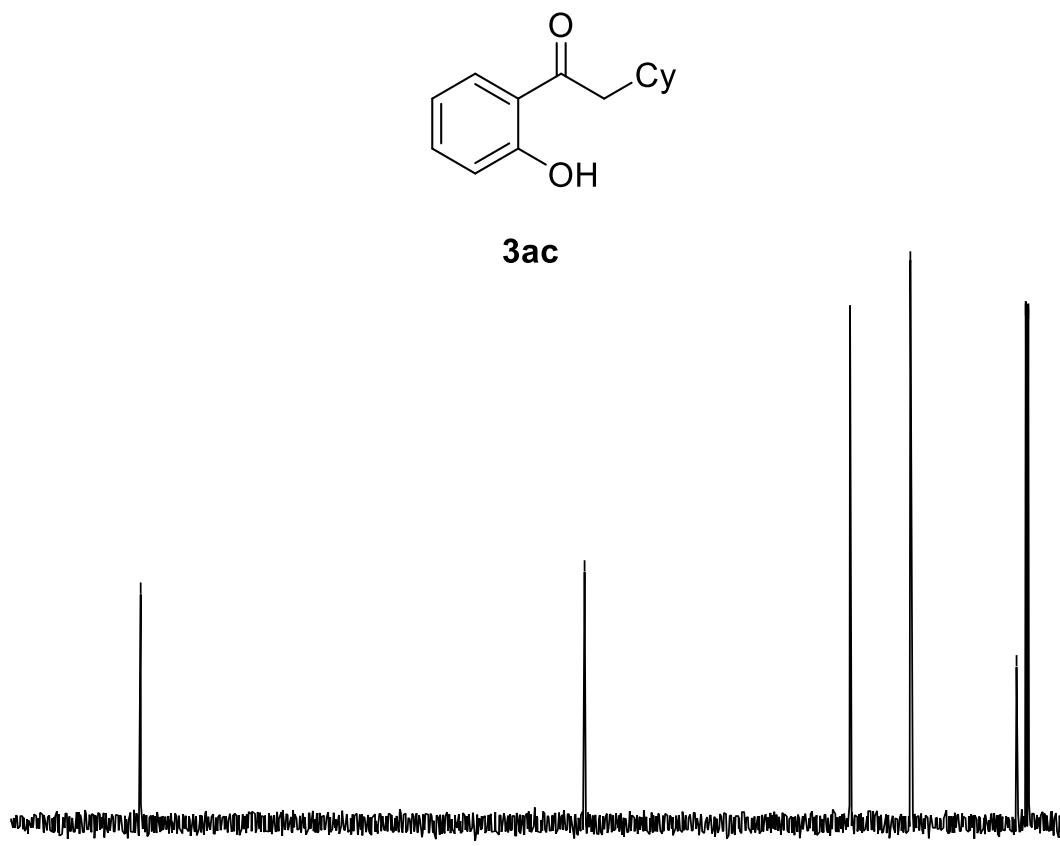

$3 a c$

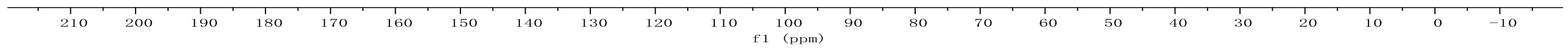

S71 


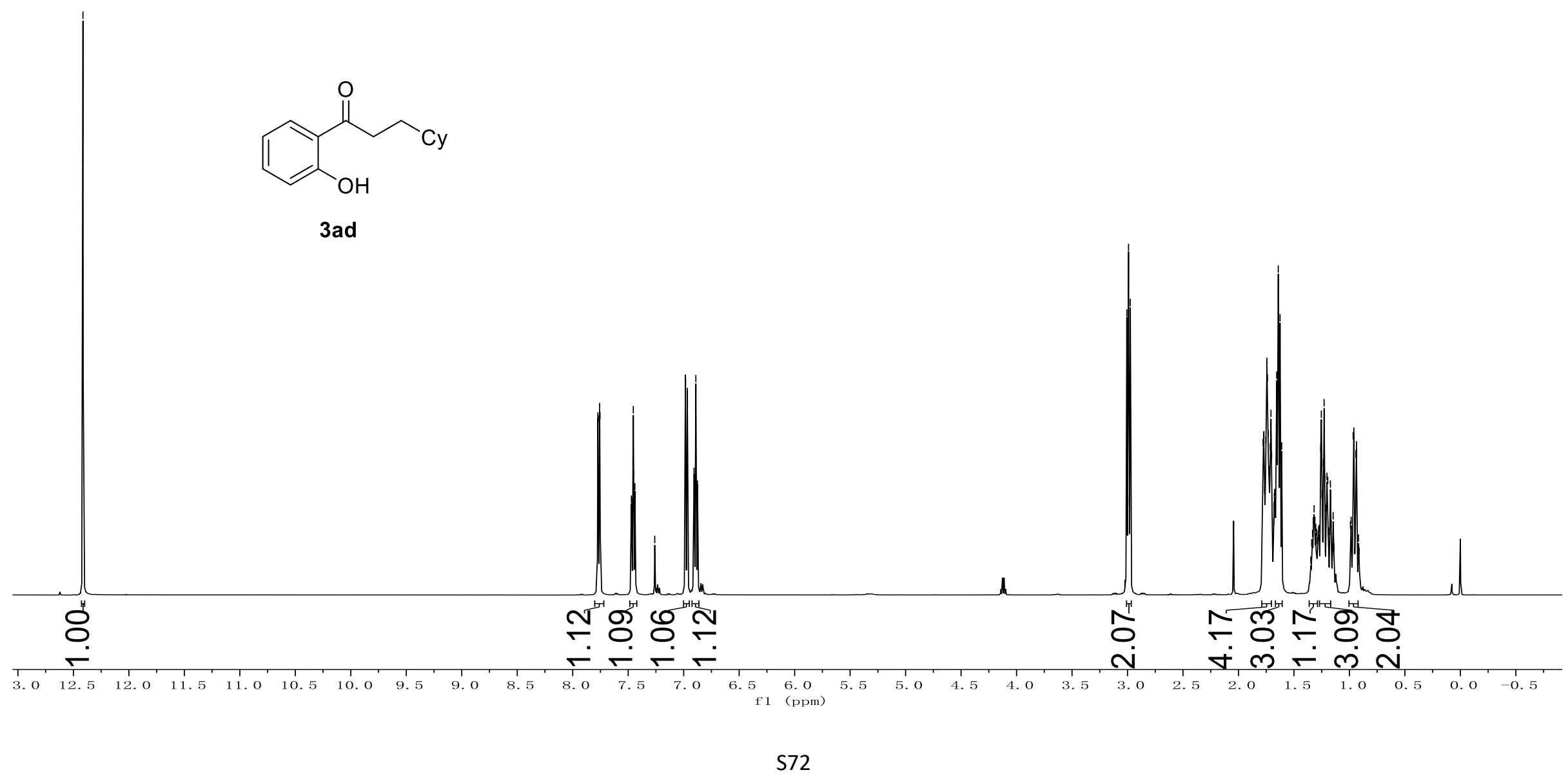


${ }^{13} \mathrm{C} \mathrm{NMR}$-spectrum $\left(126 \mathrm{MHz}, \mathrm{CDCl}_{3}\right)$ of $\mathbf{3 a d}$

惫

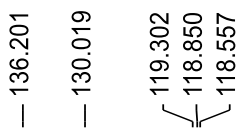

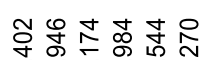

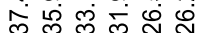

ला?

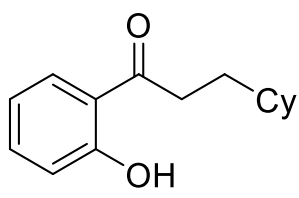

3ad

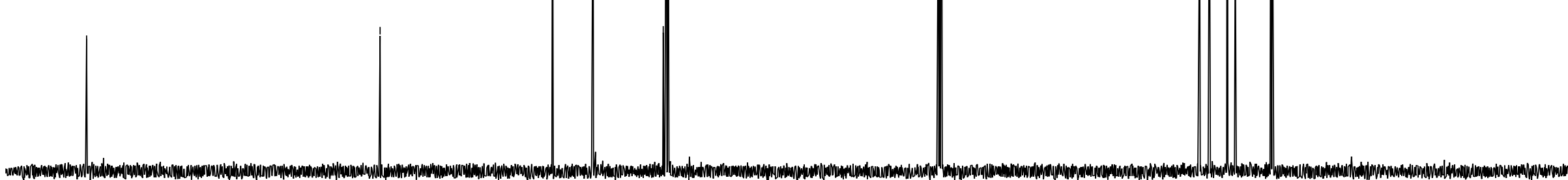

$210 \quad 200 \quad 190$
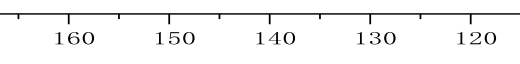

f1 $\stackrel{100}{(\mathrm{ppm})}$ 
${ }^{1} \mathrm{H}$ NMR-spectrum $\left(500 \mathrm{MHz}, \mathrm{CDCl}_{3}\right)$ of $\mathbf{3 a e}$

兌

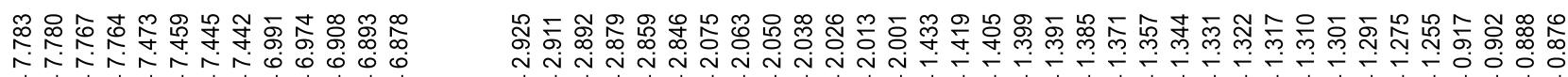

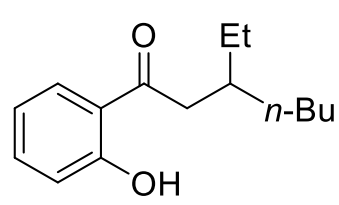

3ae

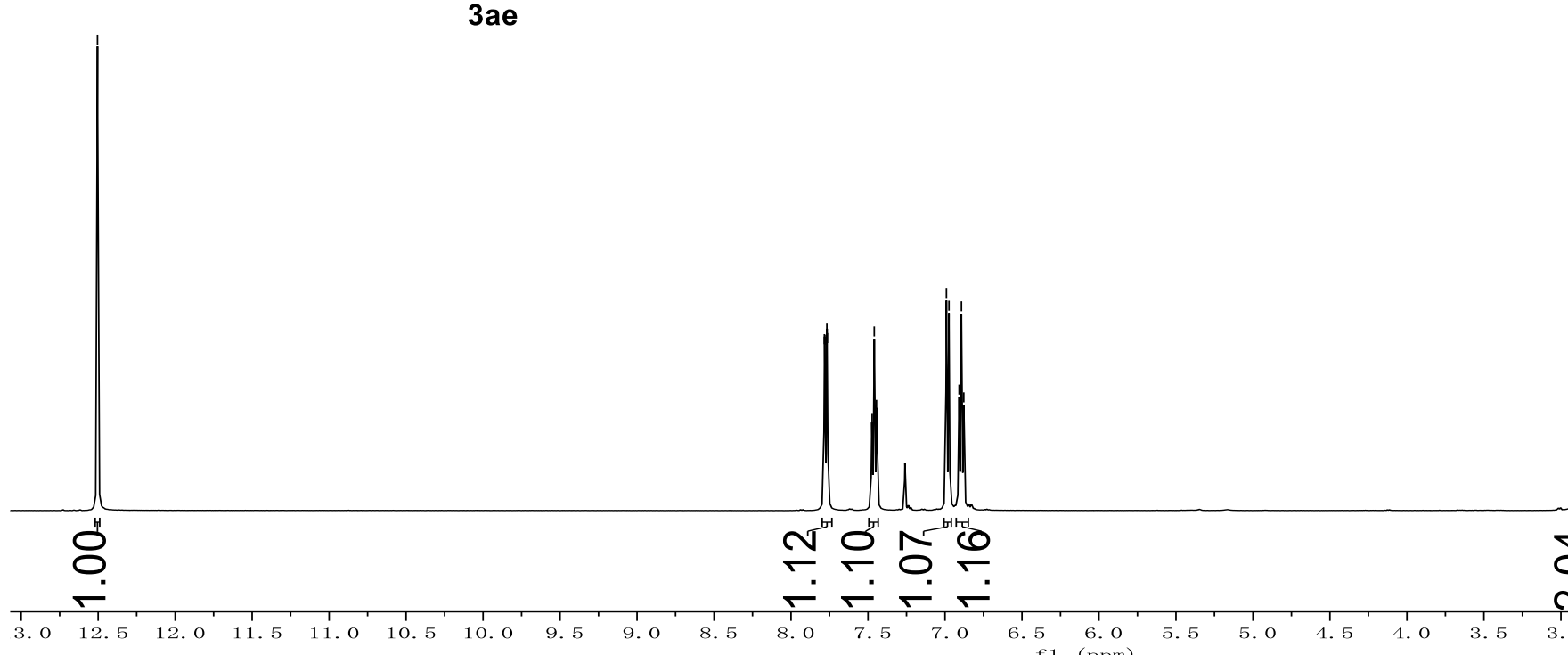
४े 
${ }^{13} \mathrm{C}$ NMR-spectrum $\left(126 \mathrm{MHz}, \mathrm{CDCl}_{3}\right)$ of $\mathbf{3 a e}$

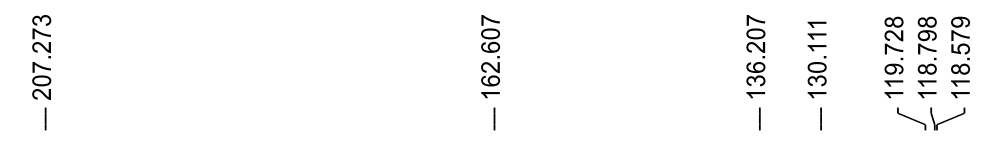

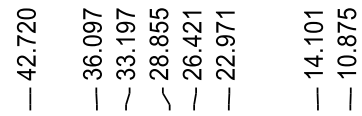

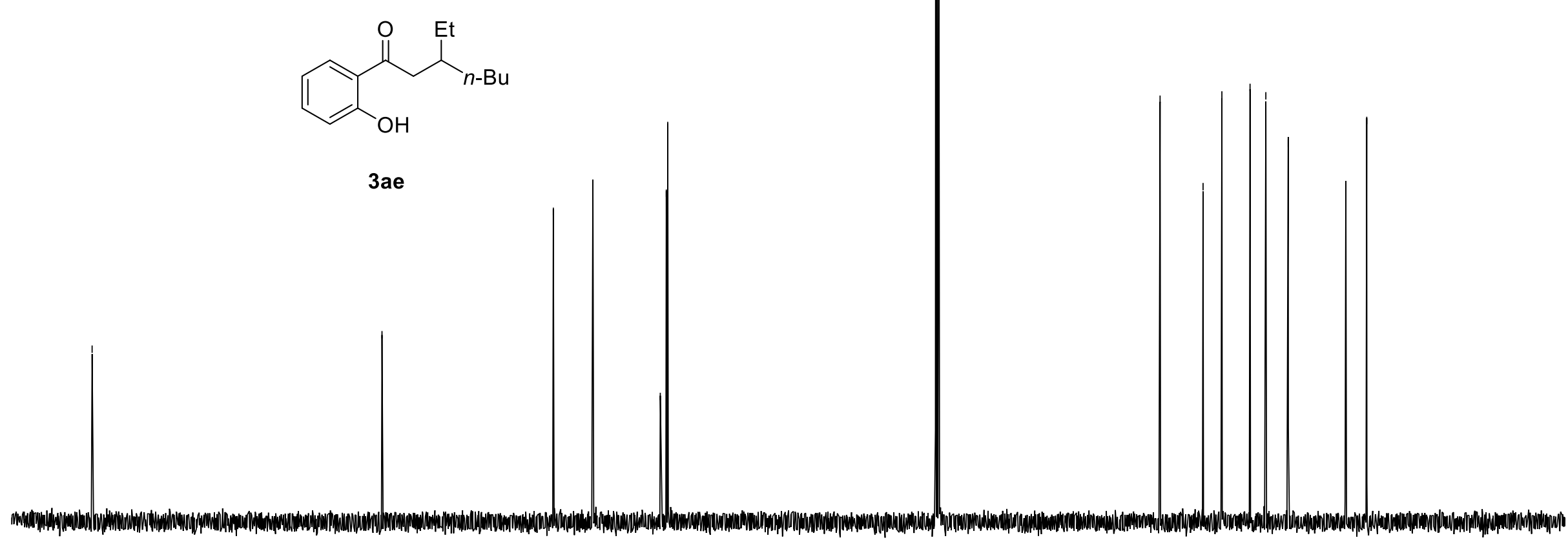

3ae

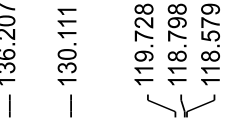

$\begin{array}{rrrr}1 & 1 & & \\ 210 & 200 & 190 & 180\end{array}$
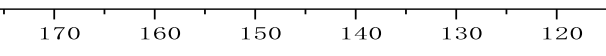

110

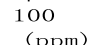


${ }^{1} \mathrm{H}$ NMR-spectrum $\left(400 \mathrm{MHz}, \mathrm{CDCl}_{3}\right.$ ) of $\mathbf{3 a f}$

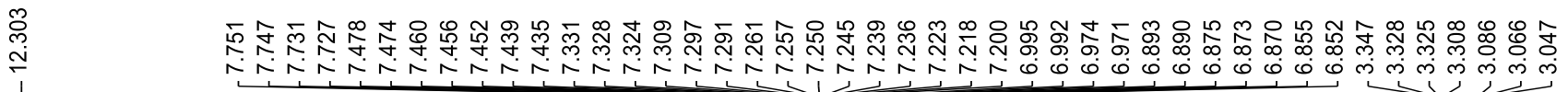
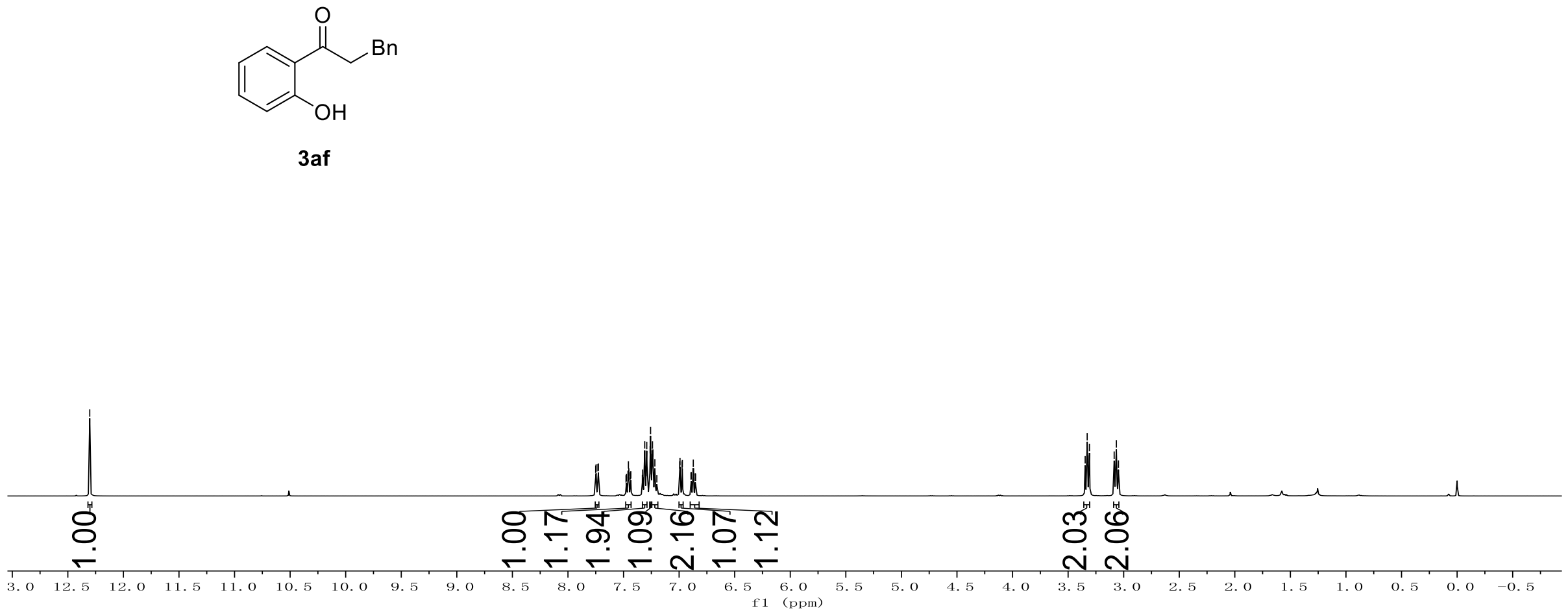
${ }^{13} \mathrm{C}$ NMR-spectrum $\left(101 \mathrm{MHz}, \mathrm{CDCl}_{3}\right)$ of $\mathbf{3 a f}$

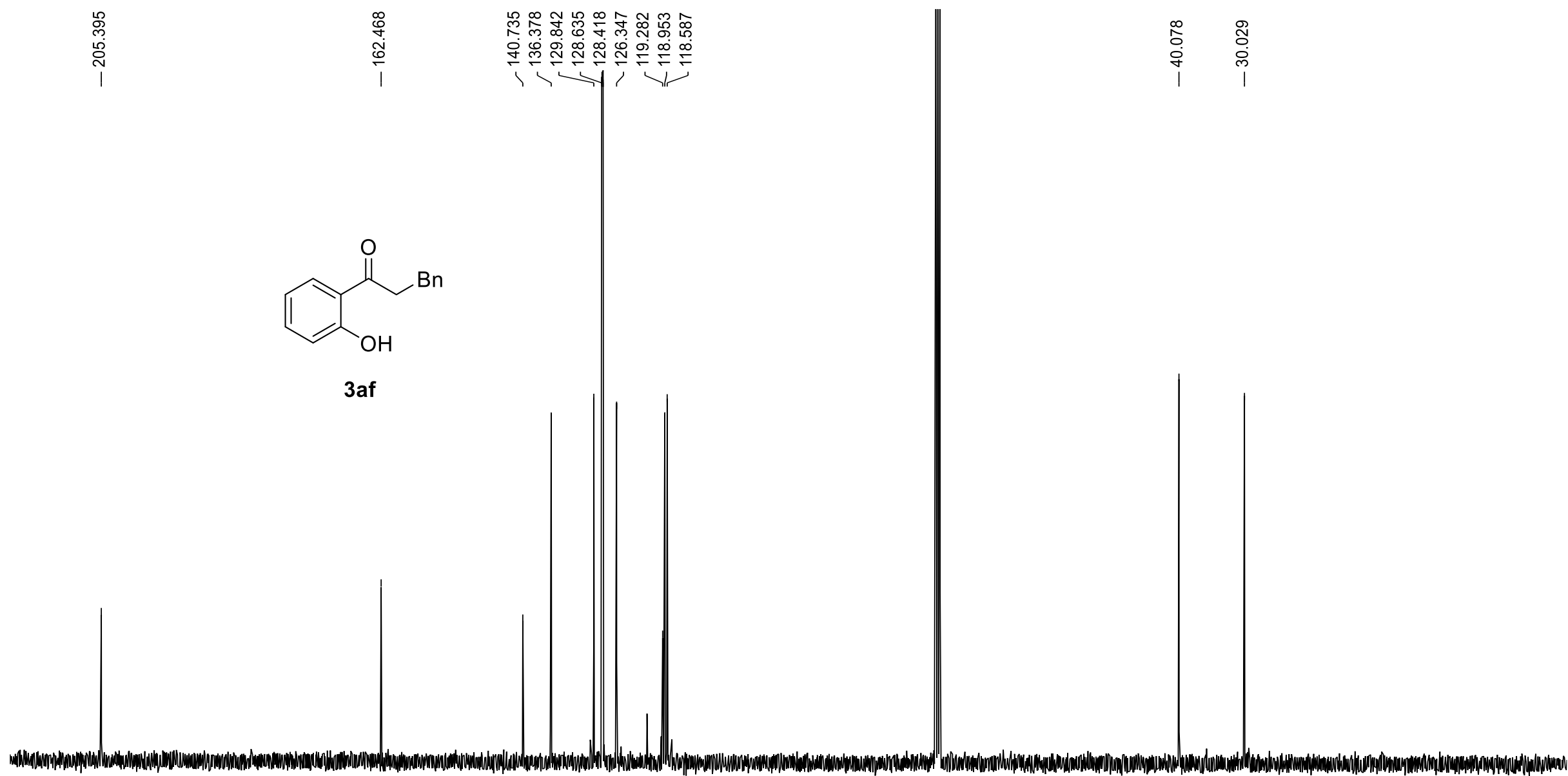


${ }^{1} \mathrm{H}$ NMR-spectrum $\left(400 \mathrm{MHz}, \mathrm{CDCl}_{3}\right)$ of $\mathbf{3 a h}$

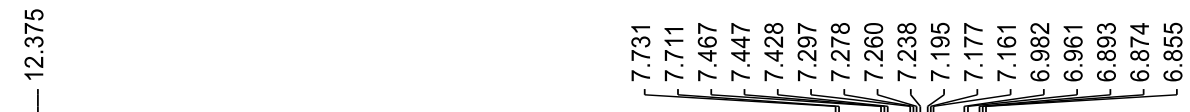

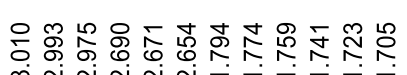

$\underbrace{m}$
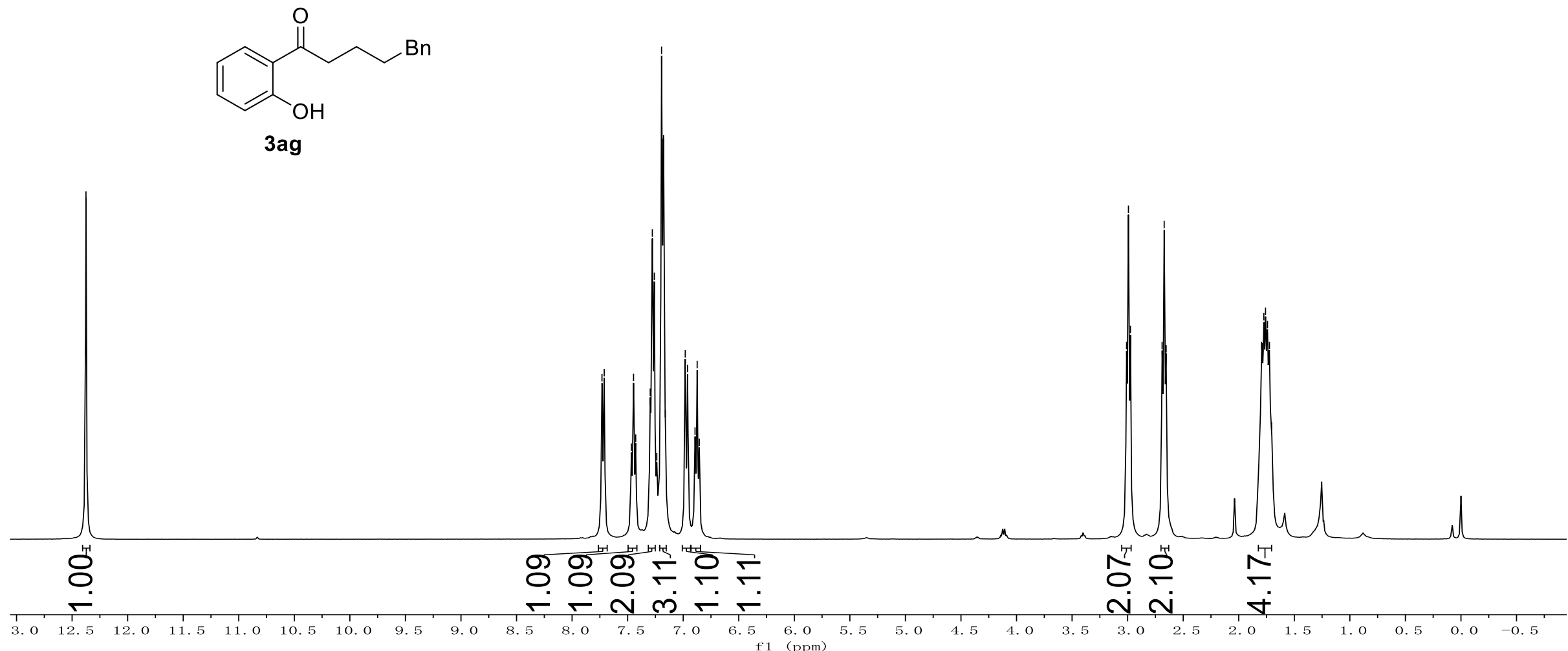
${ }^{13} \mathrm{C}$ NMR-spectrum $\left(101 \mathrm{MHz}, \mathrm{CDCl}_{3}\right)$ of $\mathbf{3 a h}$

옳

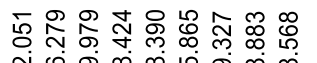

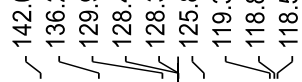

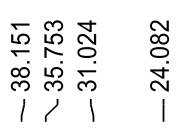

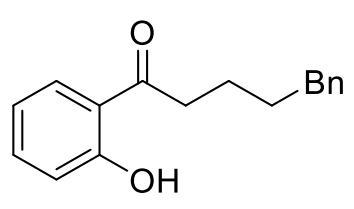

3 ag

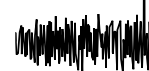
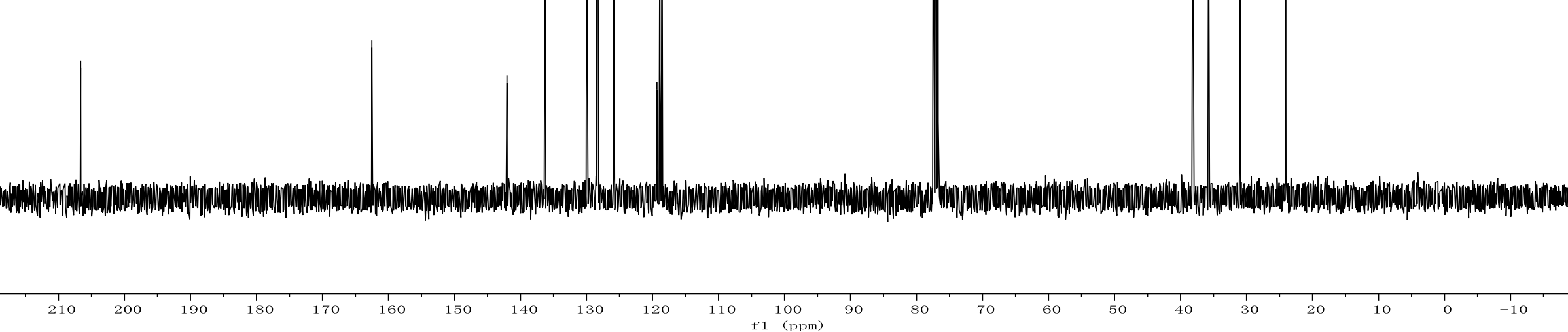
${ }^{1} \mathrm{H}$ NMR-spectrum $\left(500 \mathrm{MHz}, \mathrm{CDCl}_{3}\right)$ of $\mathbf{3 a h}$

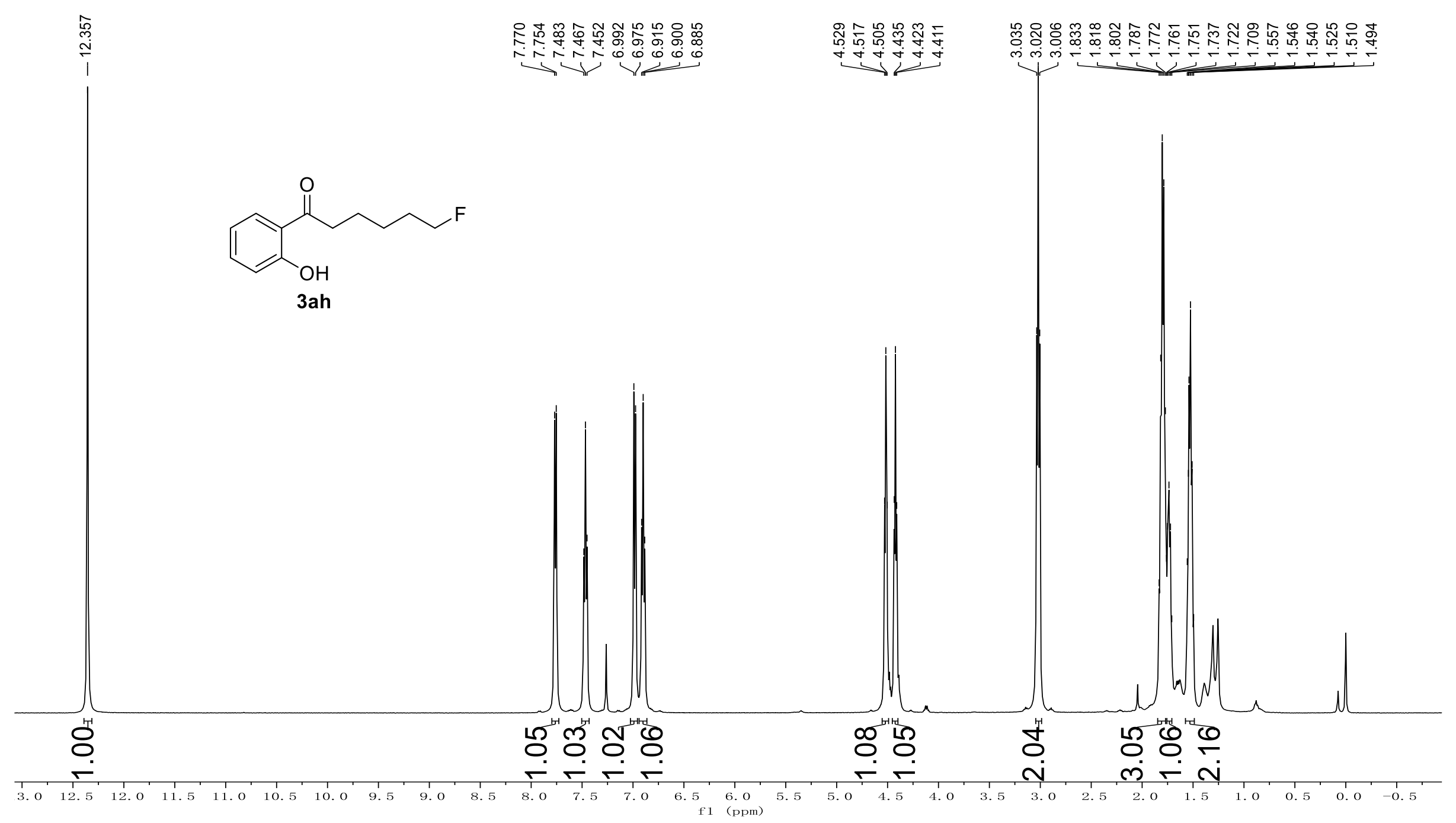


${ }^{13} \mathrm{C}$ NMR-spectrum $\left(126 \mathrm{MHz}, \mathrm{CDCl}_{3}\right)$ of $\mathbf{3 a h}$

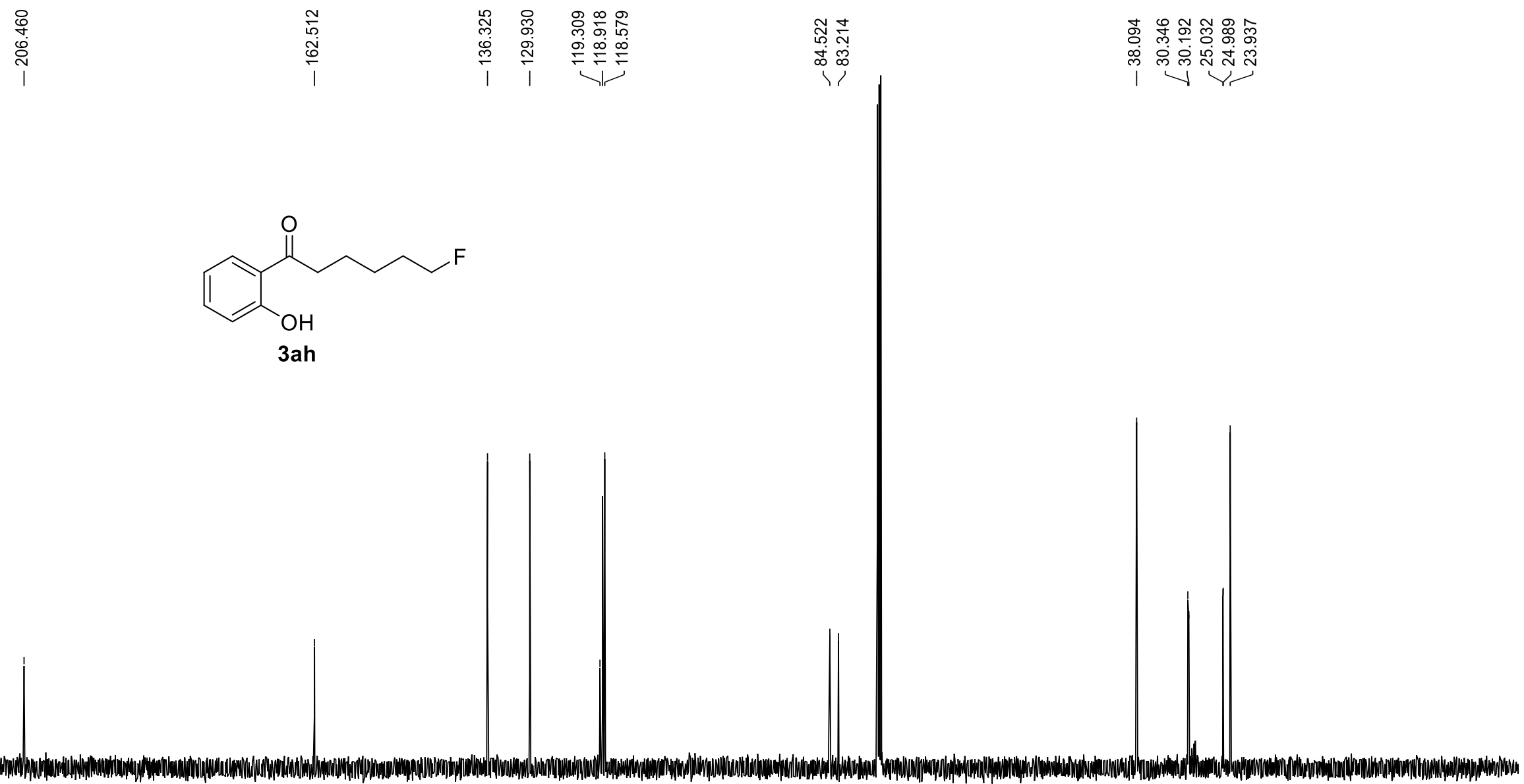

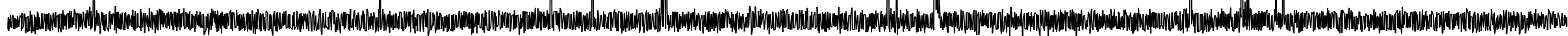

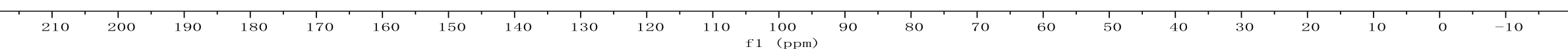


${ }^{19} \mathrm{~F}$ NMR-spectrum $\left(376 \mathrm{MHz}, \mathrm{CDCl}_{3}\right)$ of $\mathbf{3 a h}$

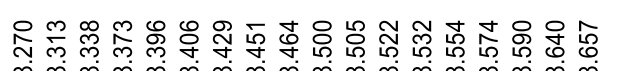

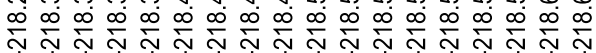
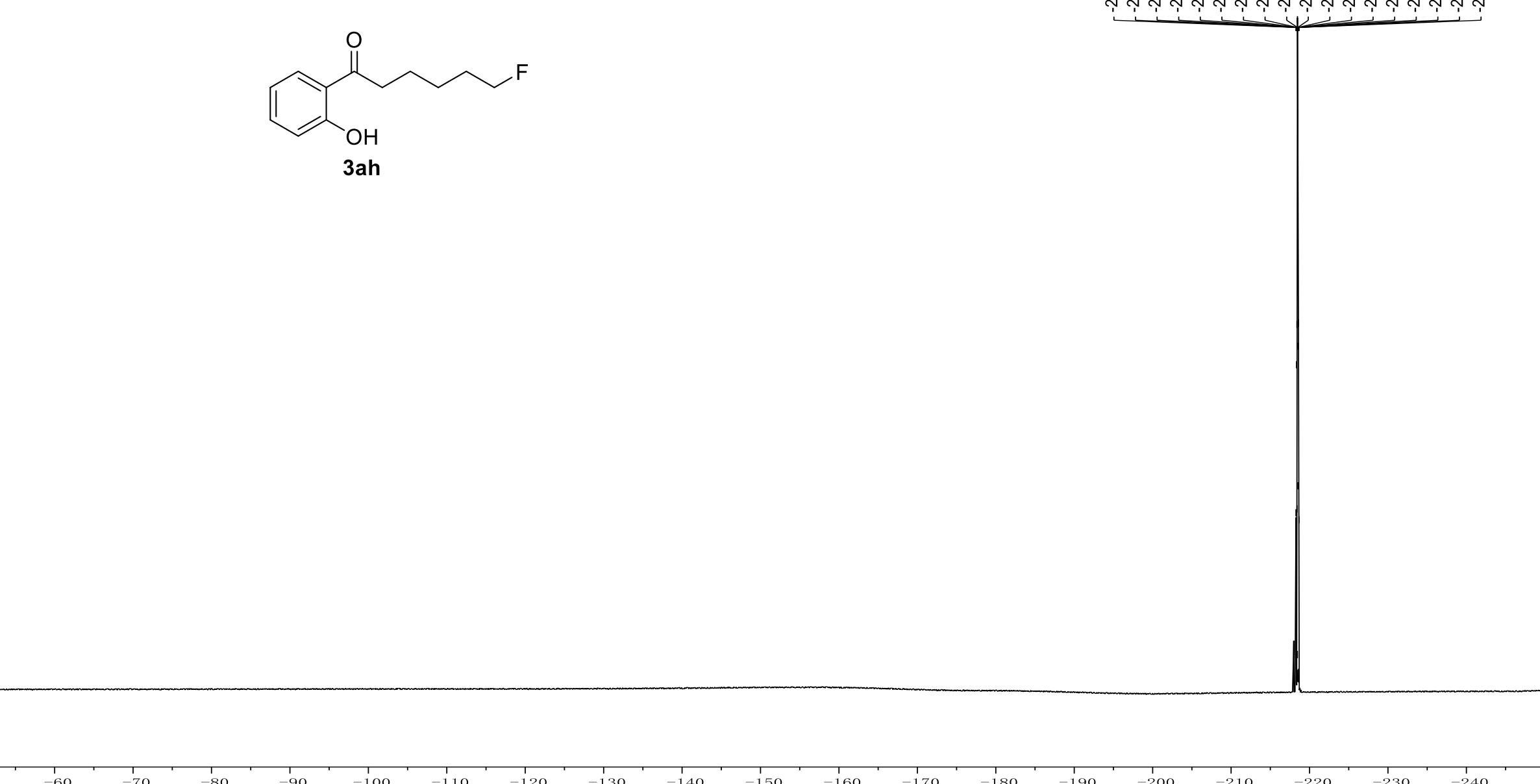

$-90 \quad-100$

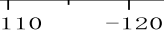

$-130$

$-150$

$-160$

$-170$

$-180 \quad-190$

$-200$

220 
${ }^{1} \mathrm{H}$ NMR-spectrum $\left(400 \mathrm{MHz}, \mathrm{CDCl}_{3}\right)$ of 3ai

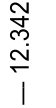

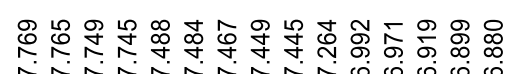

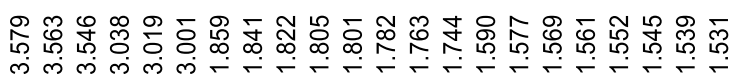

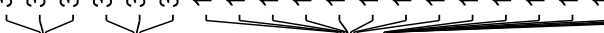
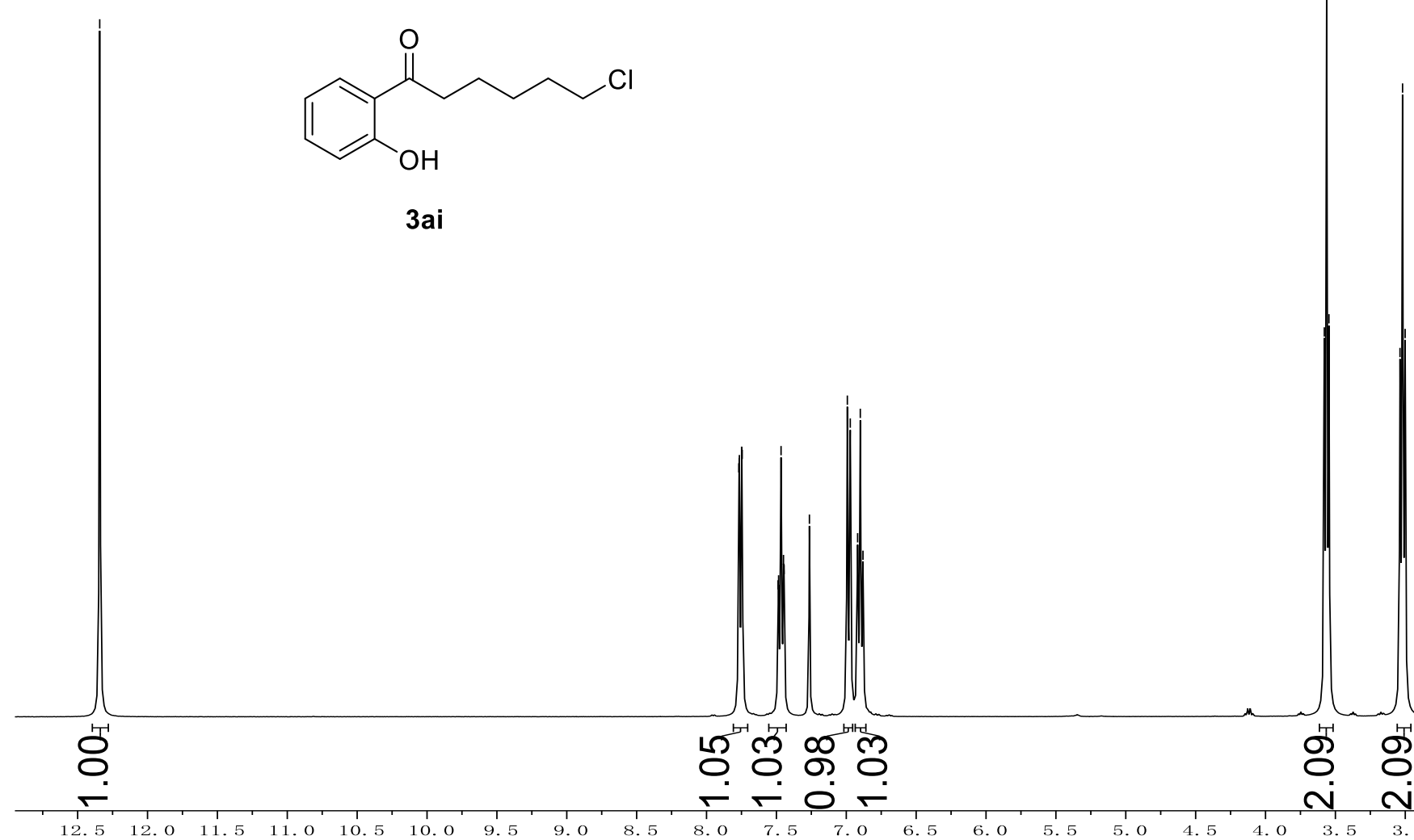

th

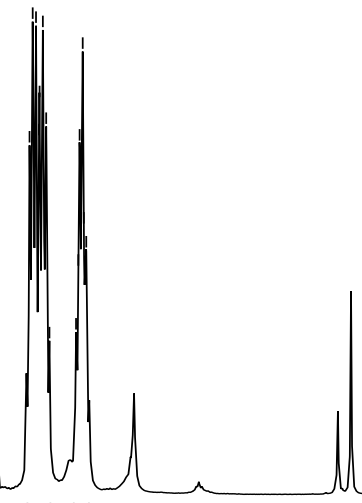

क ल

$\begin{array}{llllllllllllll}12.5 & 12.0 & 11.5 & 11.0 & 10.5 & 10.0 & 9.5 & 9.0 & 8.5 & 8.0 & 7.5 & 7.0 & 6.5 & 6.0 \\ f 1 & 6(\mathrm{pm})\end{array}$ 
${ }^{13} \mathrm{C}$ NMR-spectrum $\left(101 \mathrm{MHz}, \mathrm{CDCl}_{3}\right)$ of $\mathbf{3 a i}$

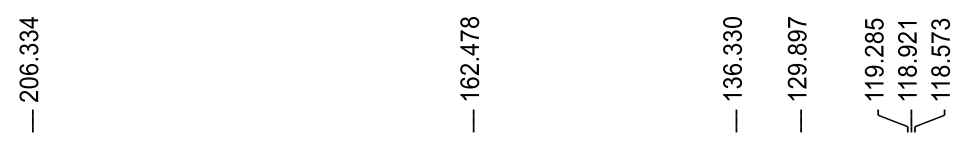

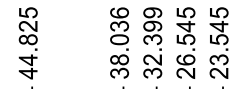

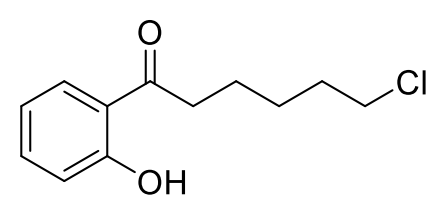

3ai
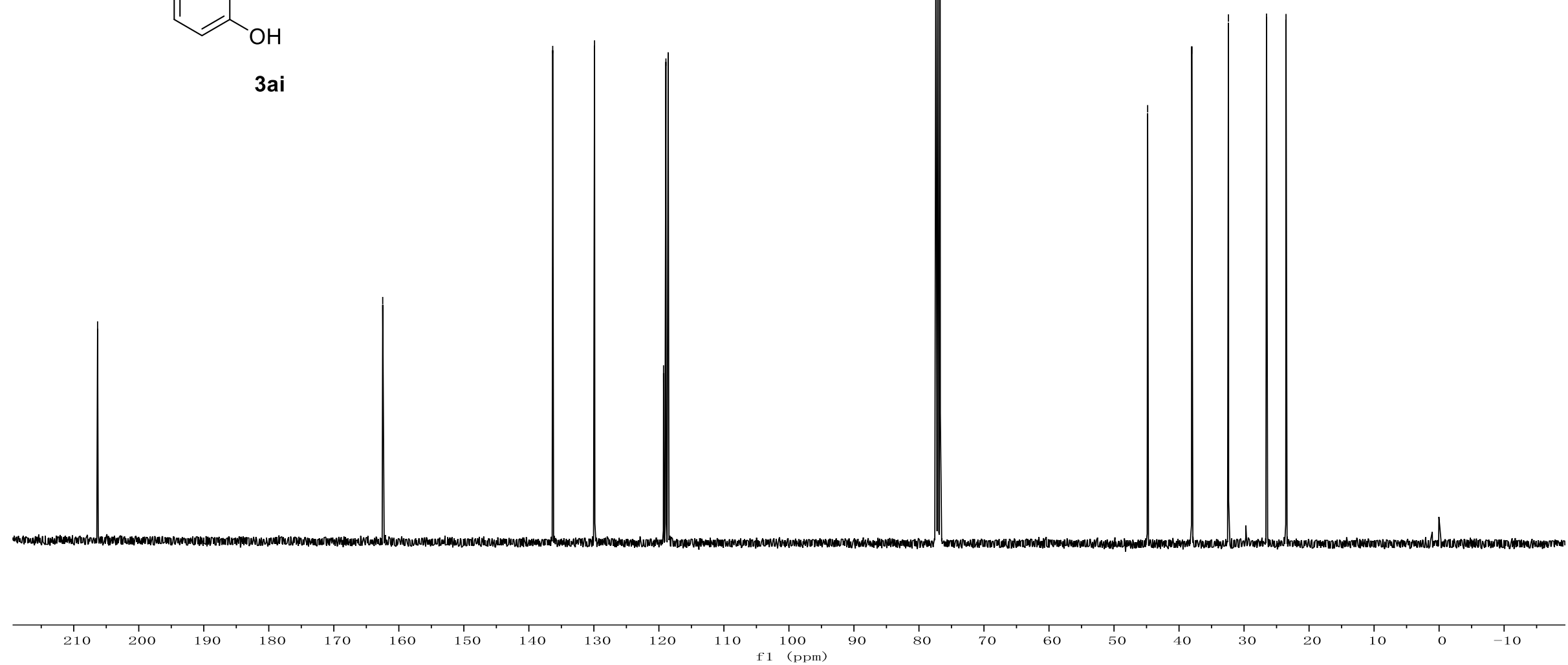
${ }^{1} \mathrm{H}$ NMR-spectrum $\left(400 \mathrm{MHz}, \mathrm{CDCl}_{3}\right)$ of 3aj

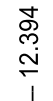

I
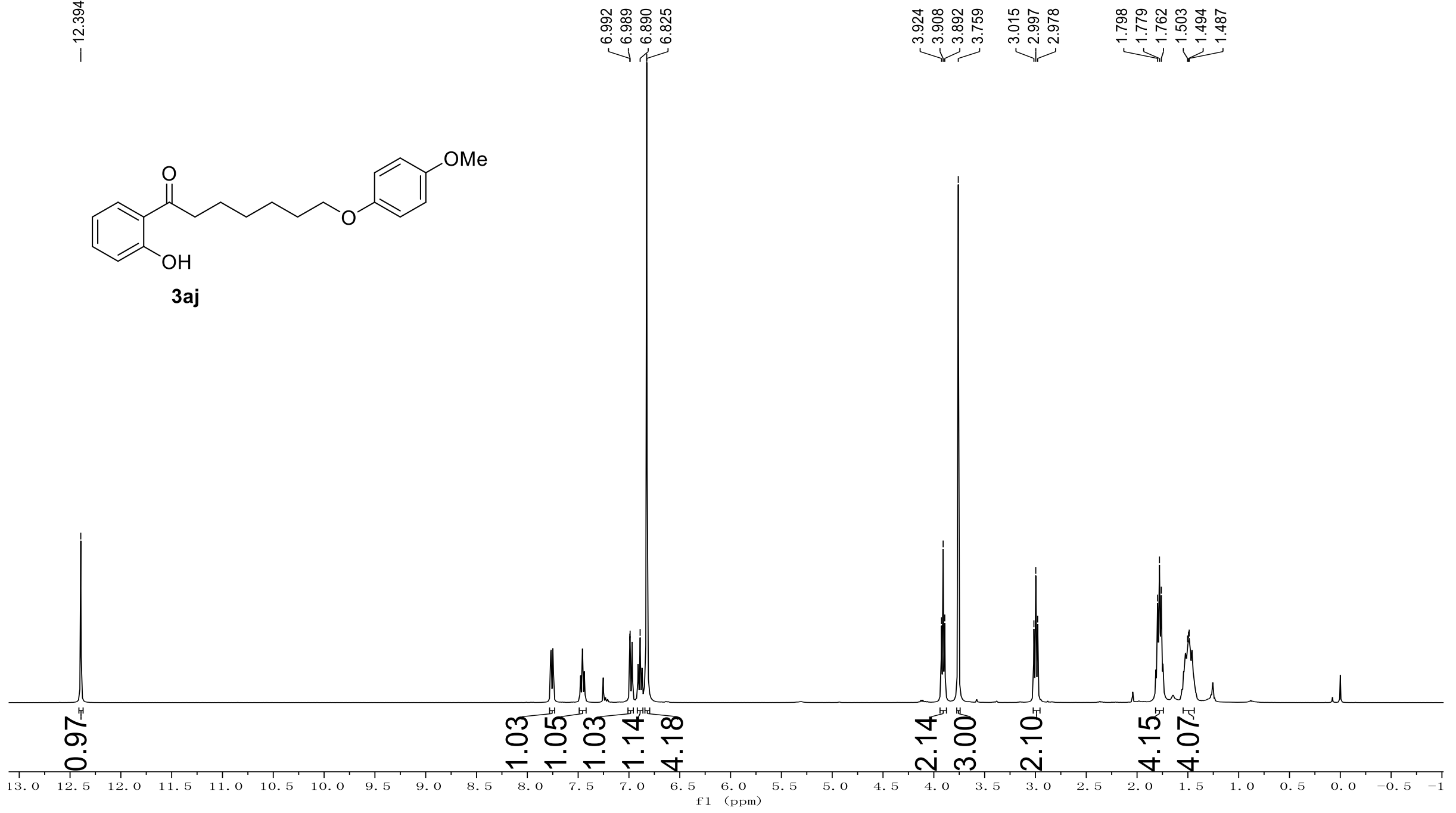
${ }^{13} \mathrm{C}$ NMR-spectrum $\left(101 \mathrm{MHz}, \mathrm{CDCl}_{3}\right)$ of $\mathbf{3 a j}$

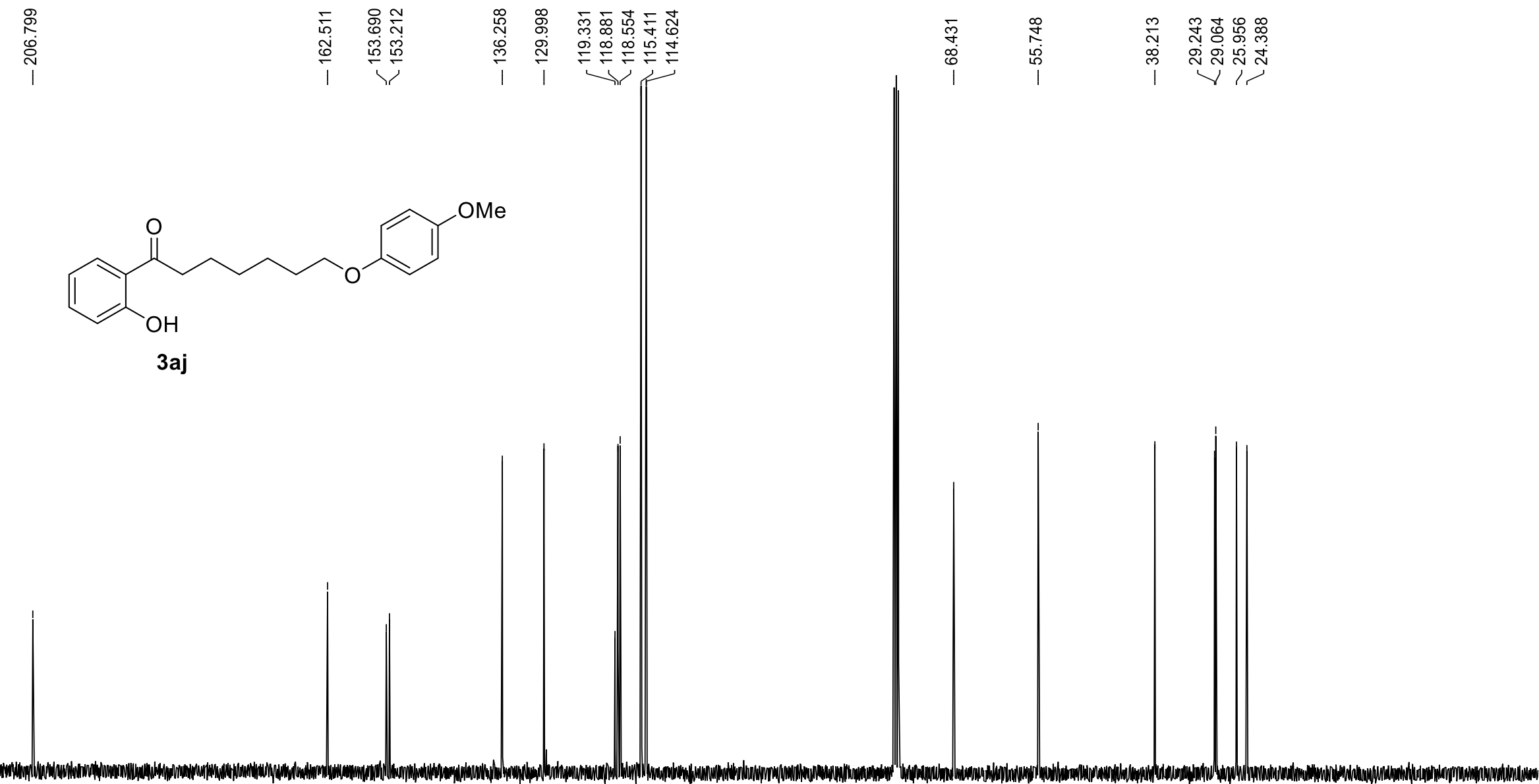

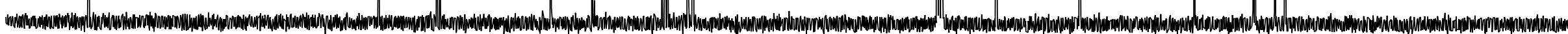

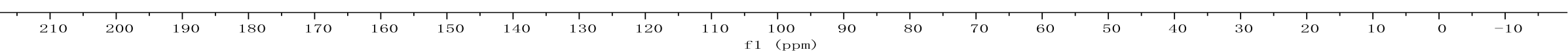


${ }^{1} \mathrm{H}$ NMR-spectrum $\left(400 \mathrm{MHz}, \mathrm{CDCl}_{3}\right)$ of $\mathbf{3 a k}$

$\stackrel{\substack{a \\ i}}{i}$

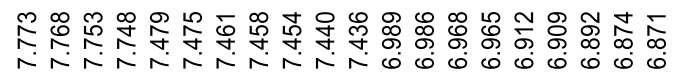

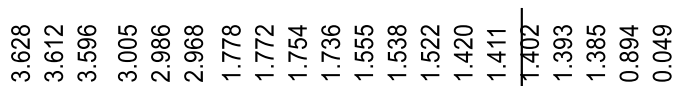

inininisin
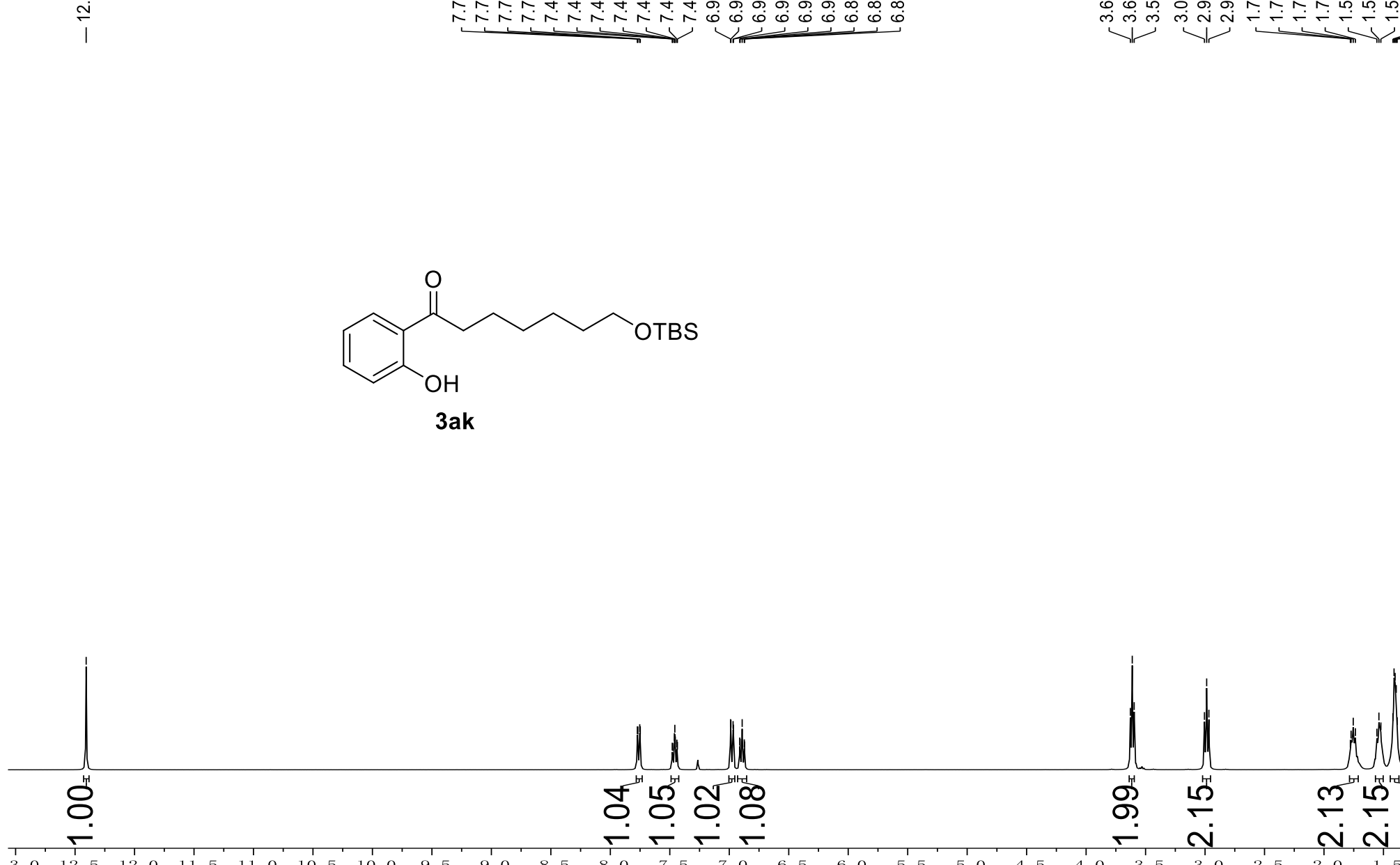
${ }^{13} \mathrm{C}$ NMR-spectrum $\left(101 \mathrm{MHz}, \mathrm{CDCl}_{3}\right)$ of $\mathbf{3 a k}$

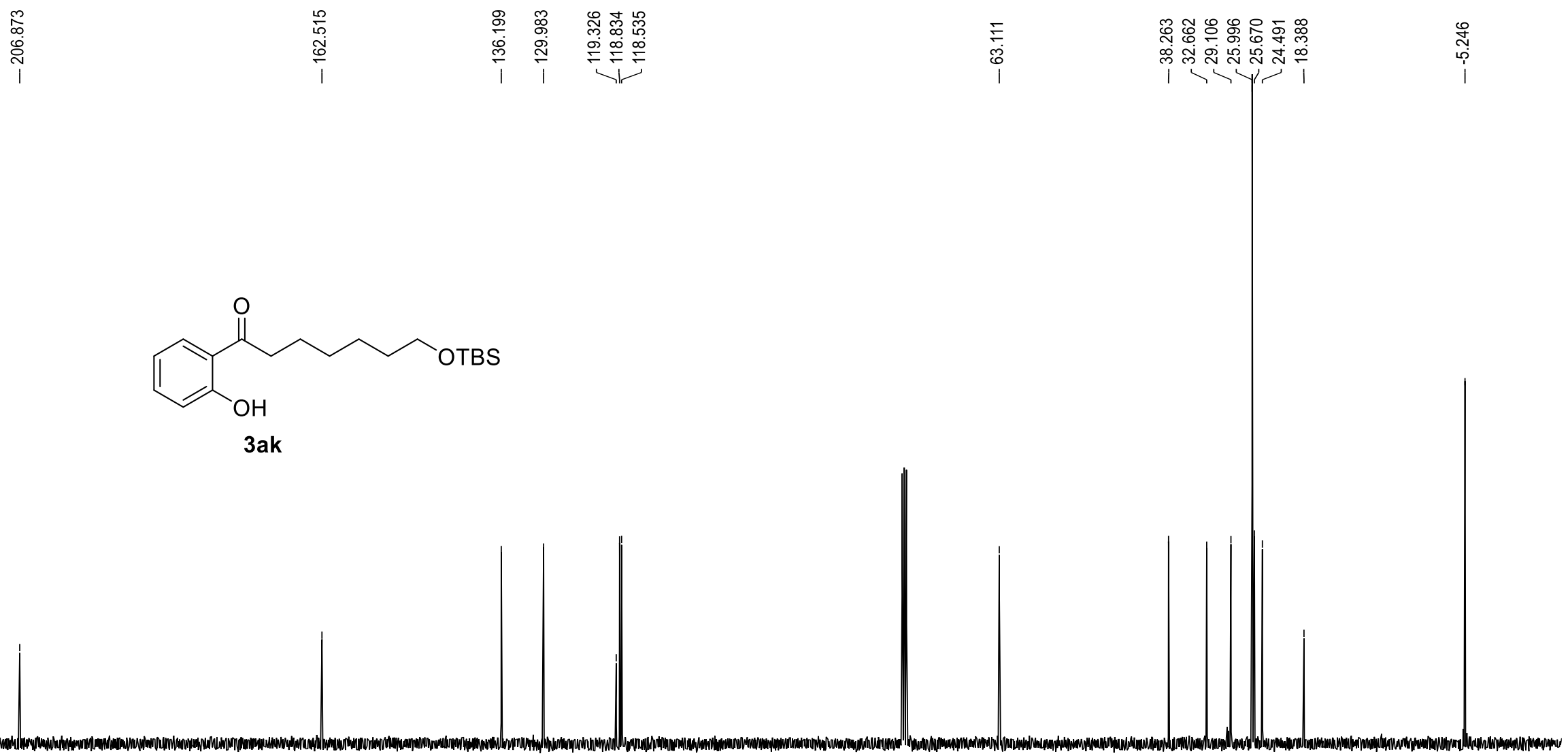

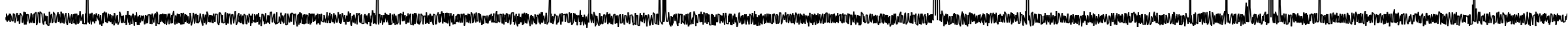


${ }^{1} \mathrm{H}$ NMR-spectrum $\left(400 \mathrm{MHz}, \mathrm{CDCl}_{3}\right.$ ) of $\mathbf{3 a l}$

$\stackrel{\infty}{\stackrel{\infty}{\mathrm{j}}}$

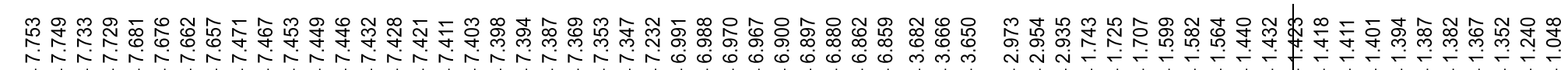

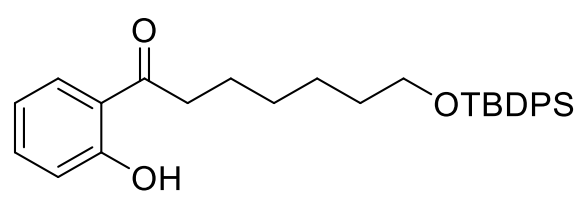

3al

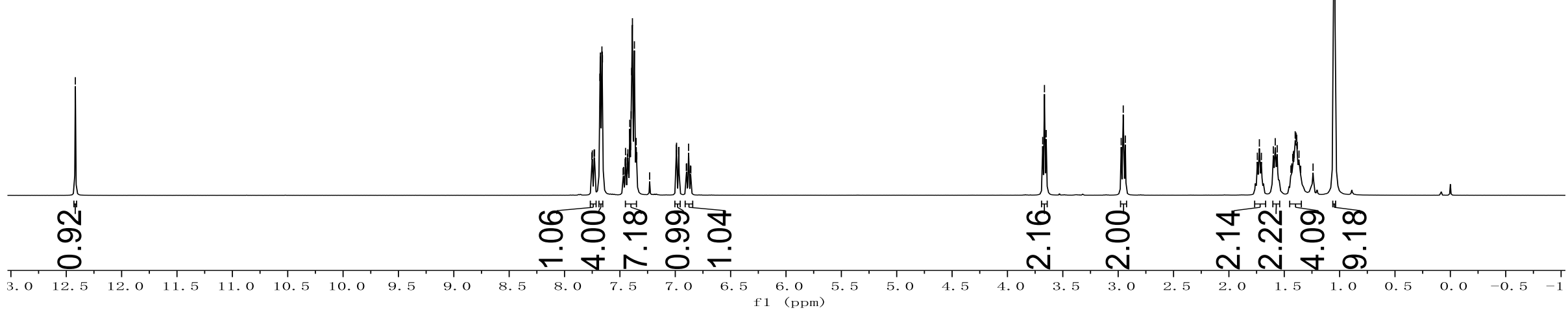


${ }^{13} \mathrm{C}$ NMR-spectrum $\left(101 \mathrm{MHz}, \mathrm{CDCl}_{3}\right)$ of 3al
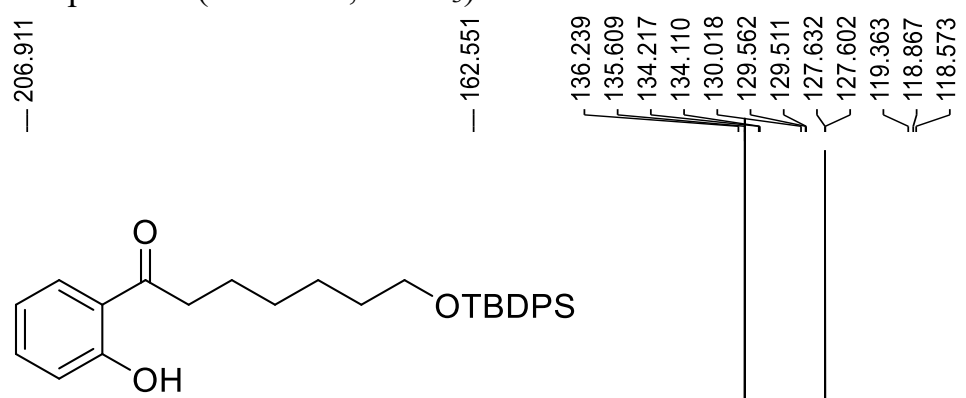

3al
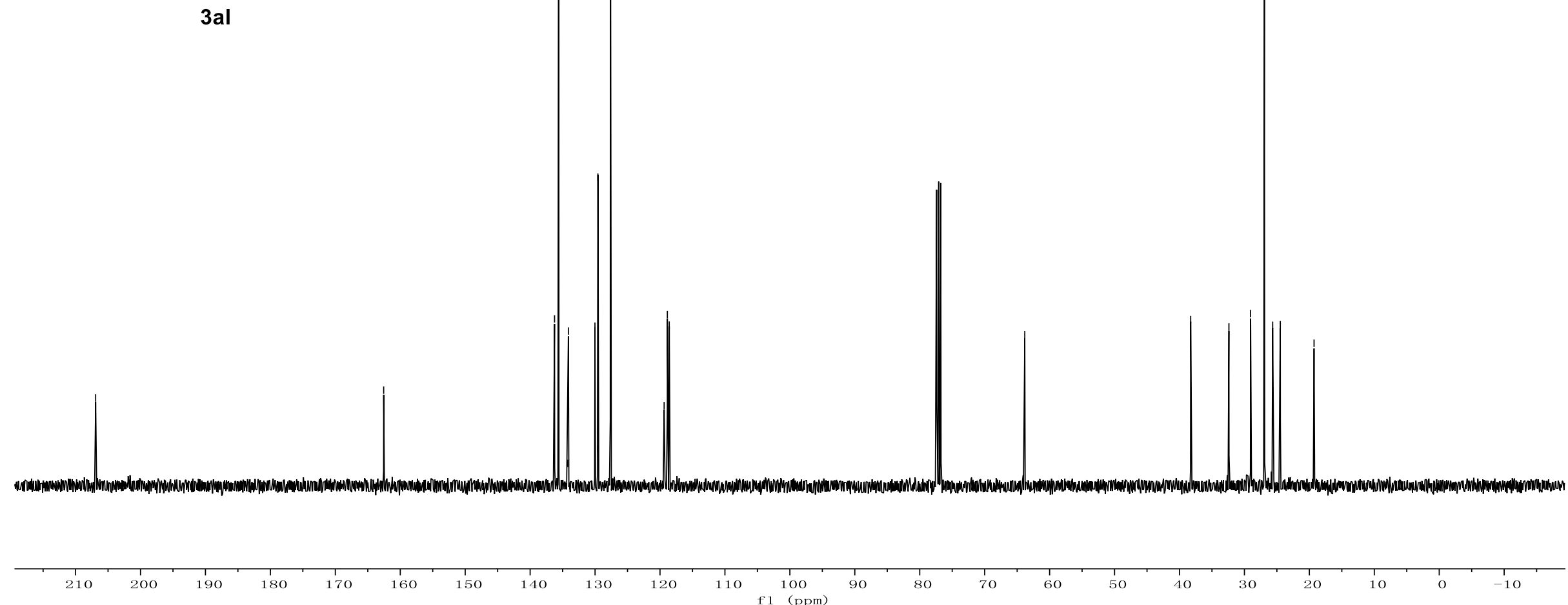

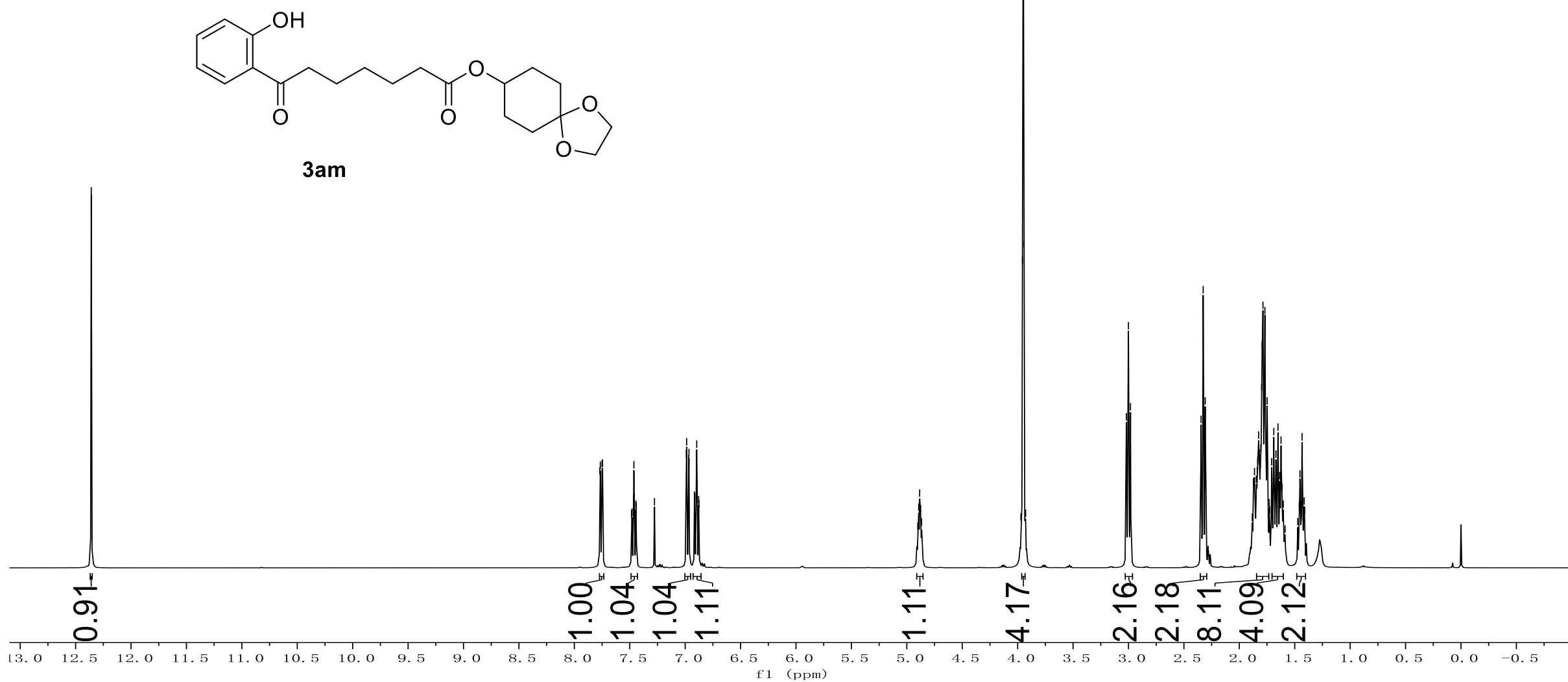
${ }^{13} \mathrm{C}$ NMR-spectrum $\left(101 \mathrm{MHz}, \mathrm{CDCl}_{3}\right)$ of $\mathbf{3 a m}$

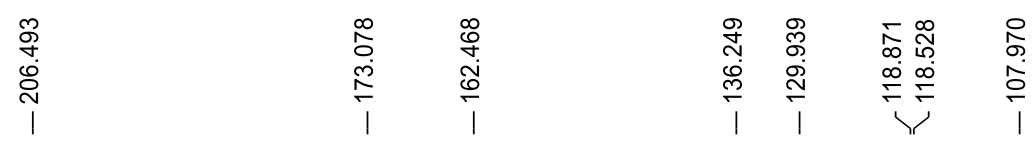

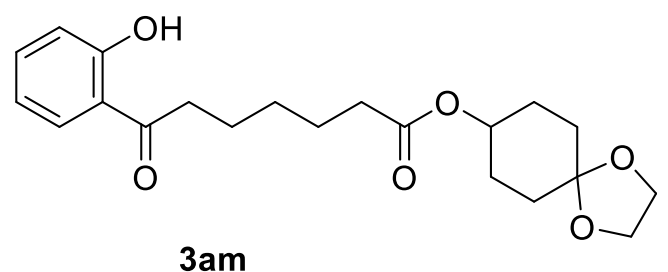

$3 a m$
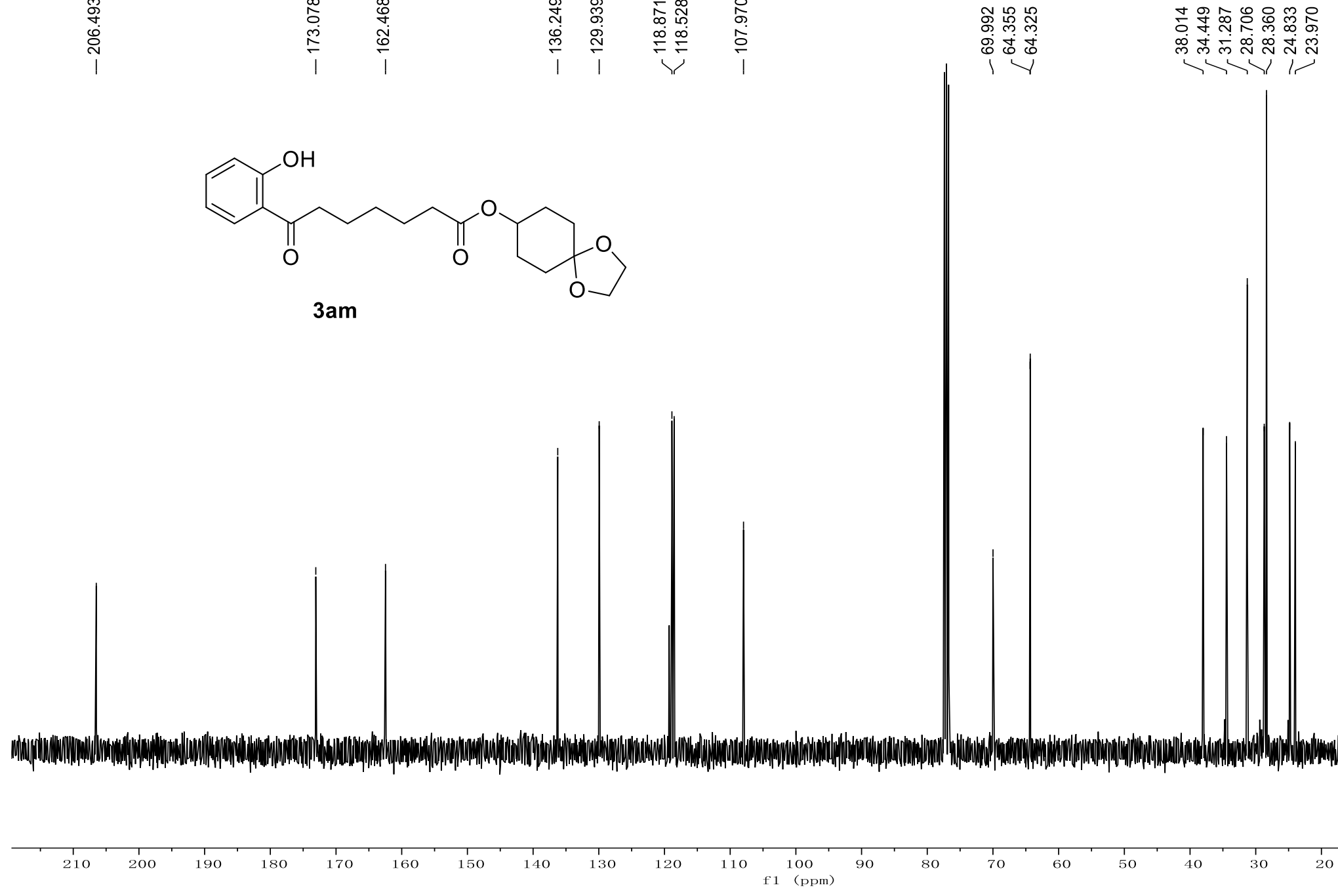
${ }^{1} \mathrm{H}$ NMR-spectrum $\left(500 \mathrm{MHz}, \mathrm{CDCl}_{3}\right)$ of $\mathbf{3 a n}$

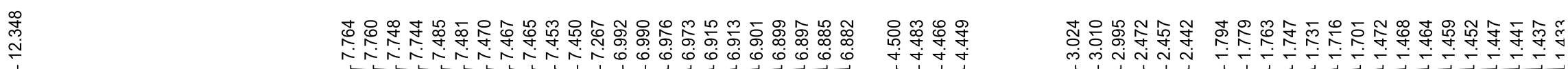
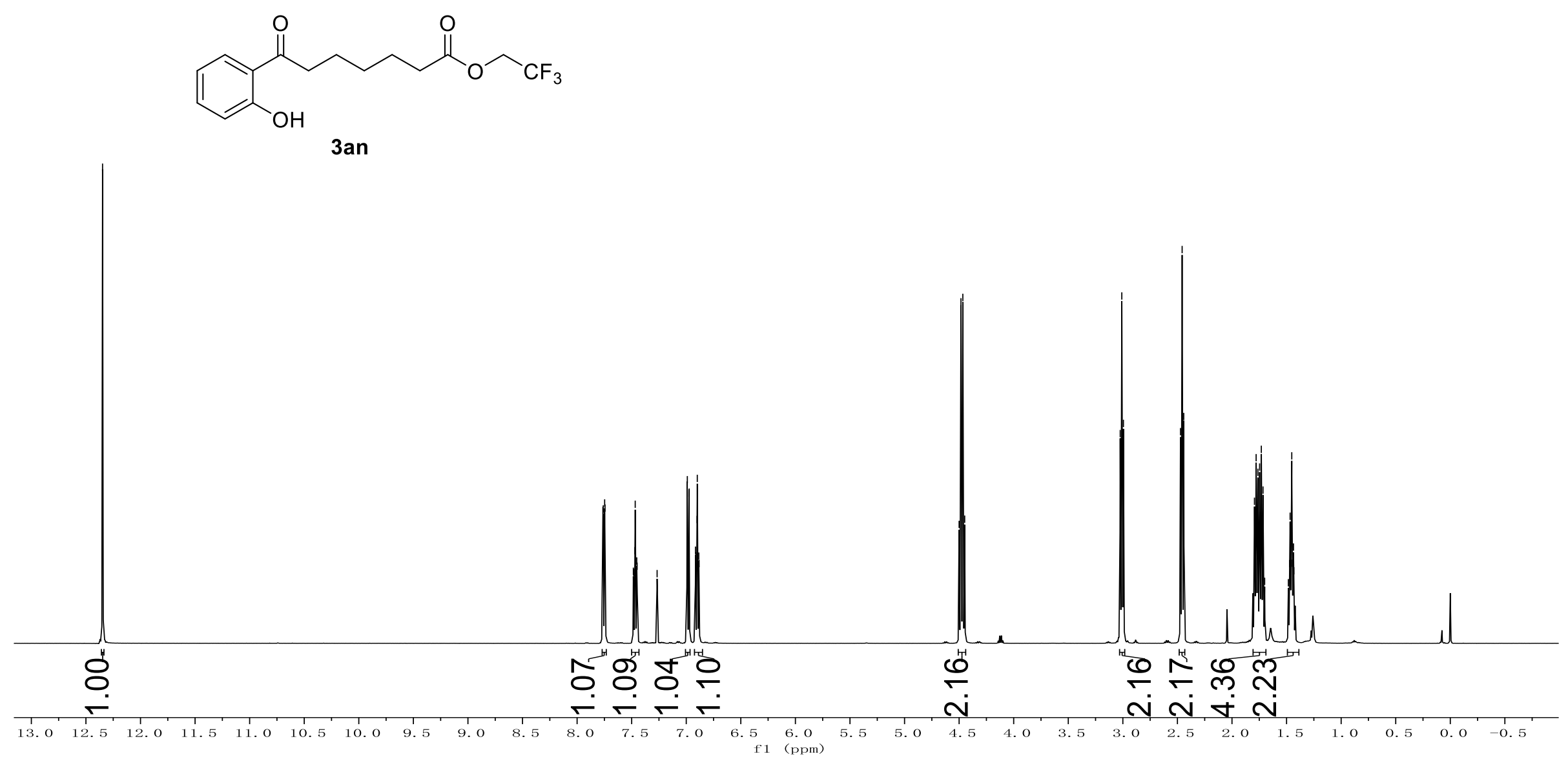
${ }^{13} \mathrm{C}$ NMR-spectrum $\left(126 \mathrm{MHz}, \mathrm{CDCl}_{3}\right)$ of $\mathbf{3 a n}$

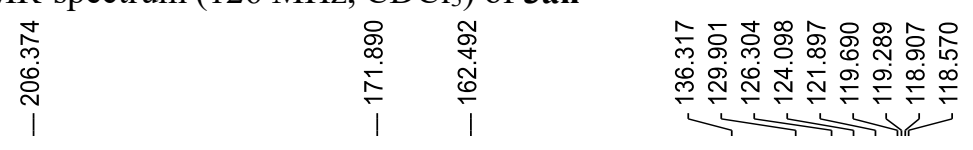

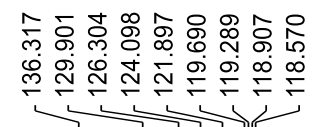

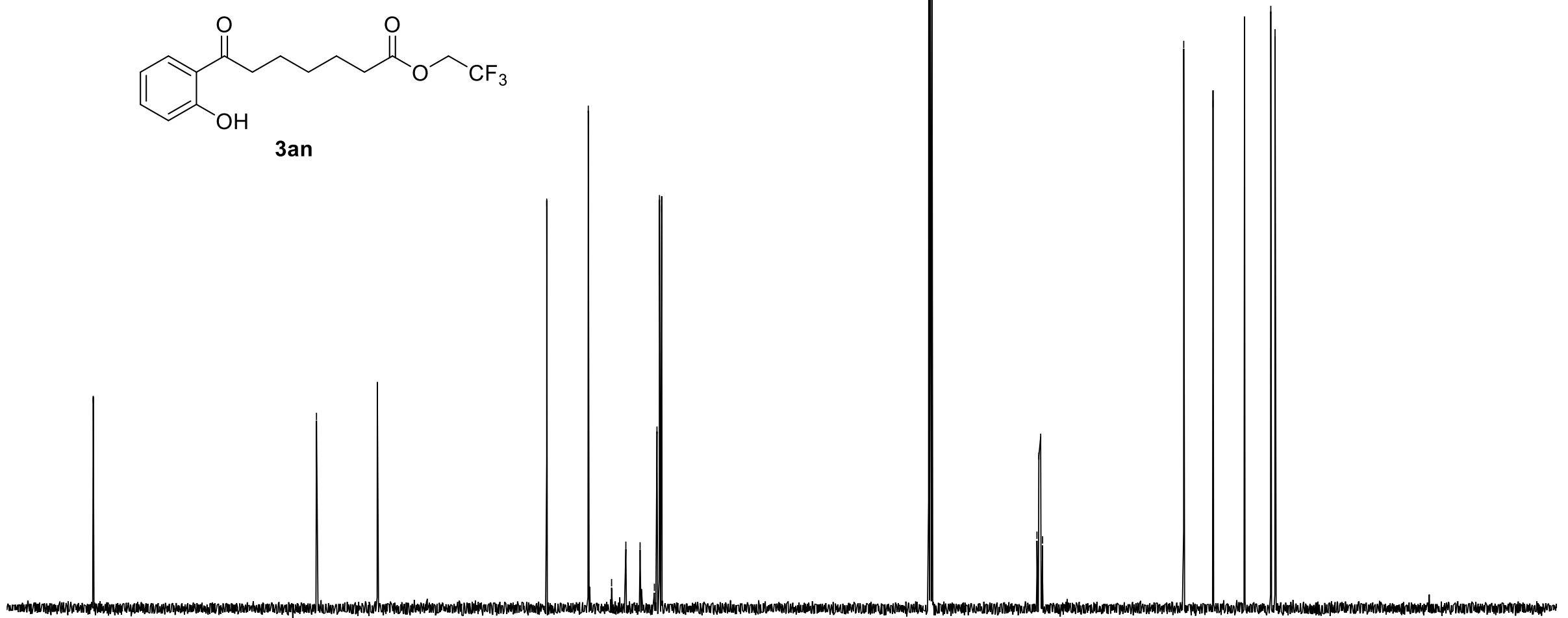

3an
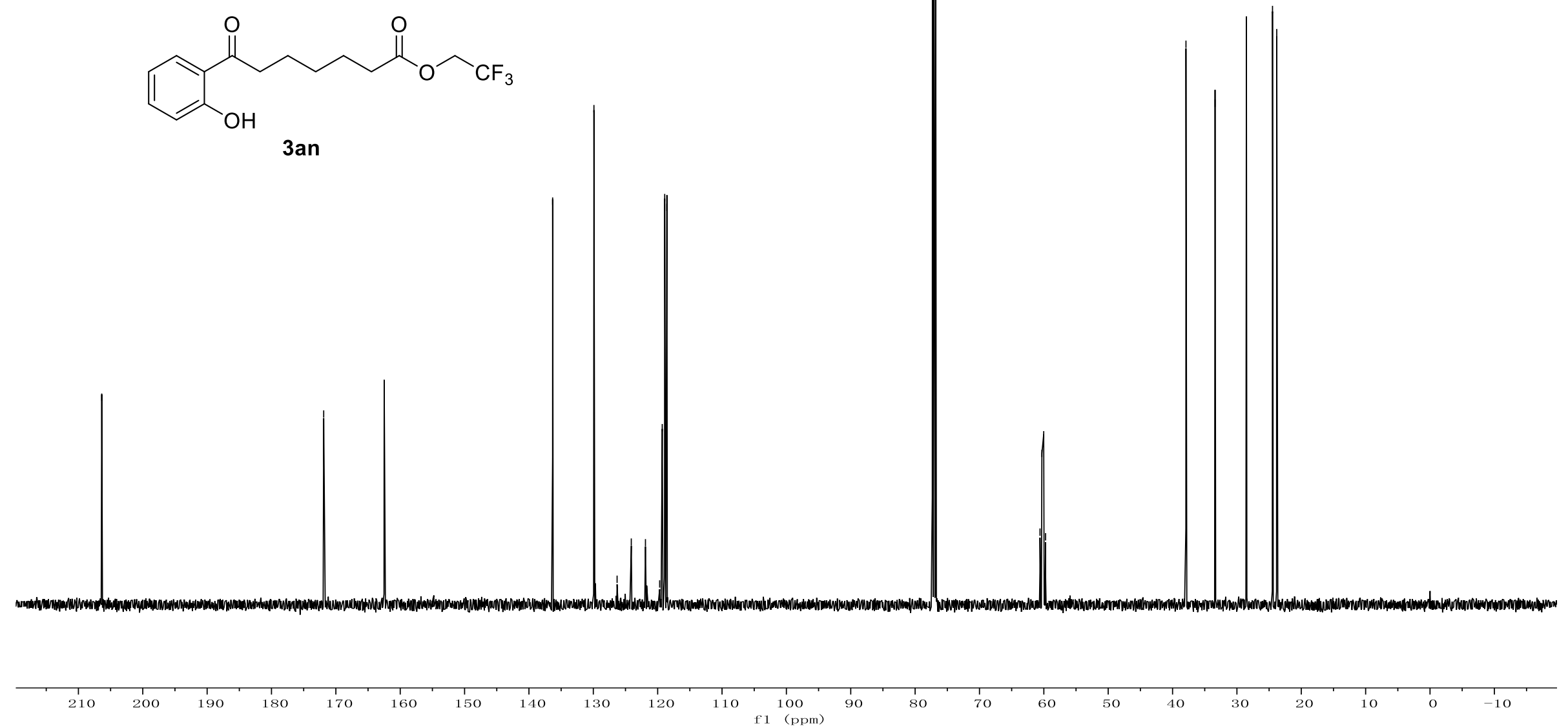
${ }^{19} \mathrm{~F}$ NMR-spectrum $\left(376 \mathrm{MHz}, \mathrm{CDCl}_{3}\right)$ of 3 an

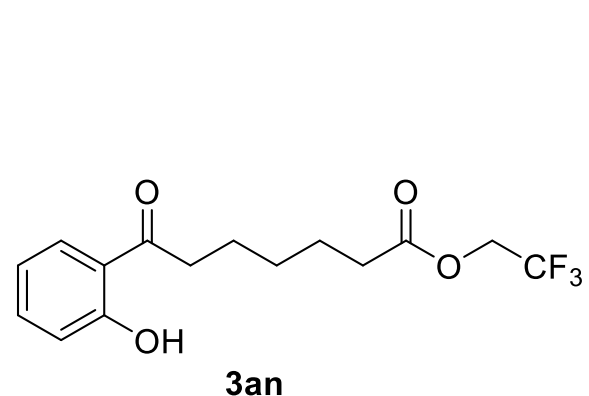

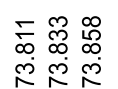

定实

$3 a n$

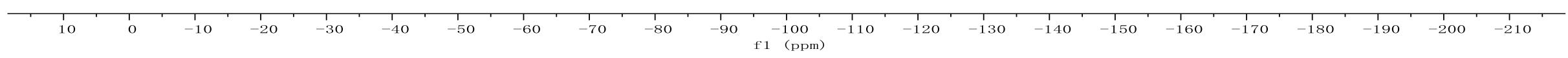


${ }^{1} \mathrm{H}$ NMR-spectrum $\left(500 \mathrm{MHz}, \mathrm{CDCl}_{3}\right)$ of $\mathbf{3 a o}$

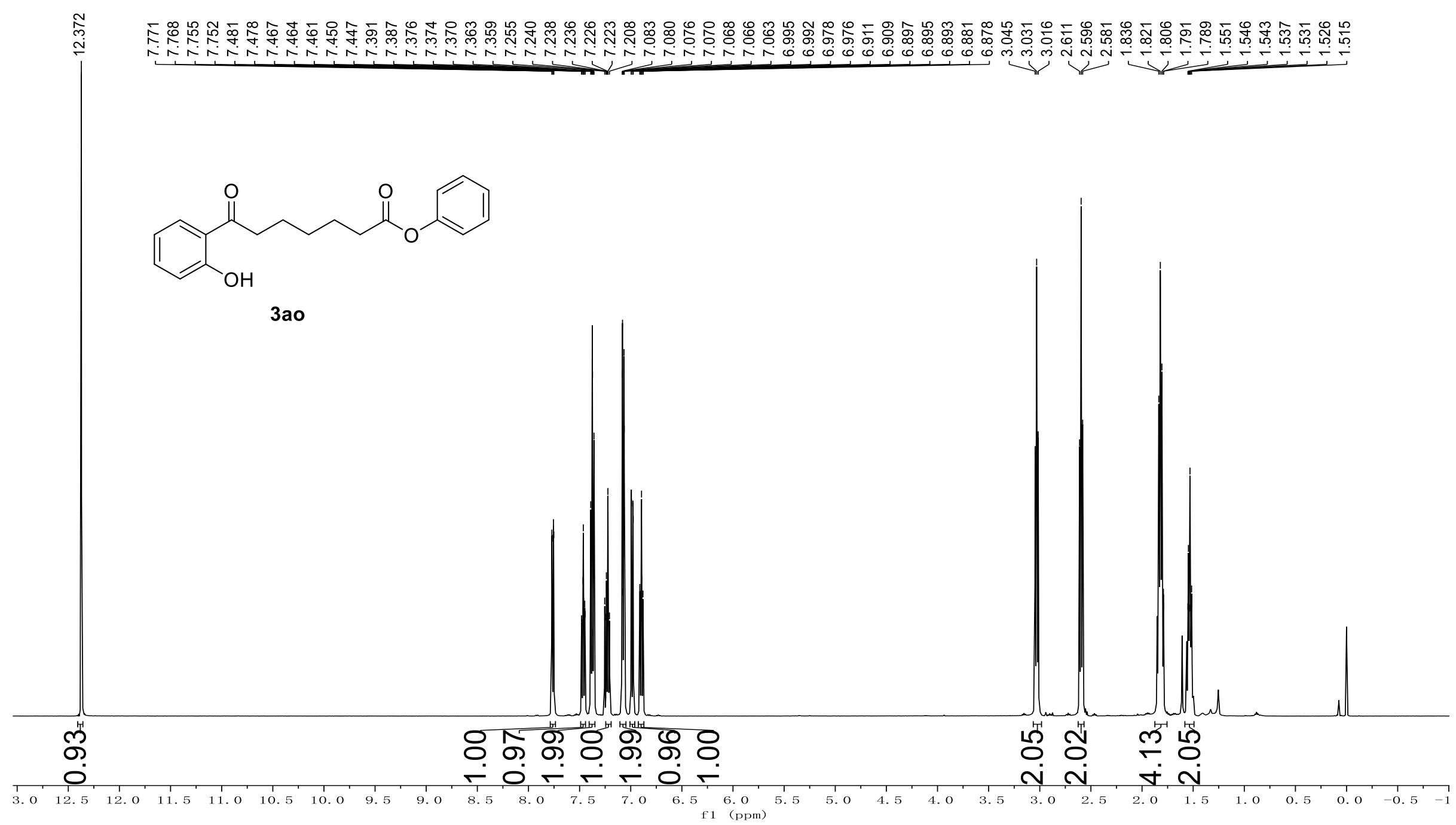


${ }^{13} \mathrm{C}$ NMR-spectrum $\left(126 \mathrm{MHz}, \mathrm{CDCl}_{3}\right)$ of $\mathbf{3 a o}$
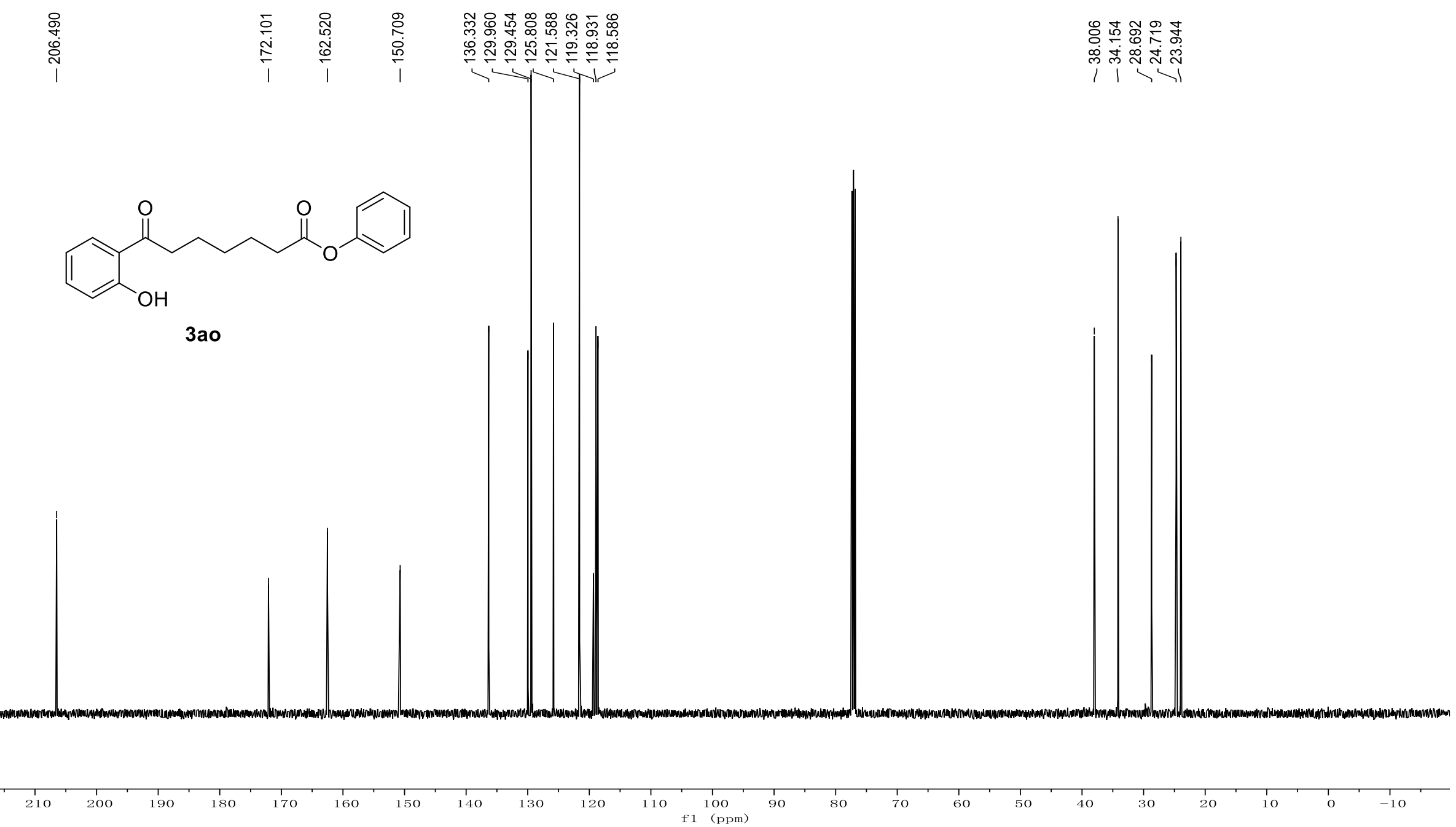
${ }^{1} \mathrm{H}$ NMR-spectrum (400 MHz, $\mathrm{CDCl}_{3}$ ) of 3ap

$$
\text { ్ㅠำ }
$$
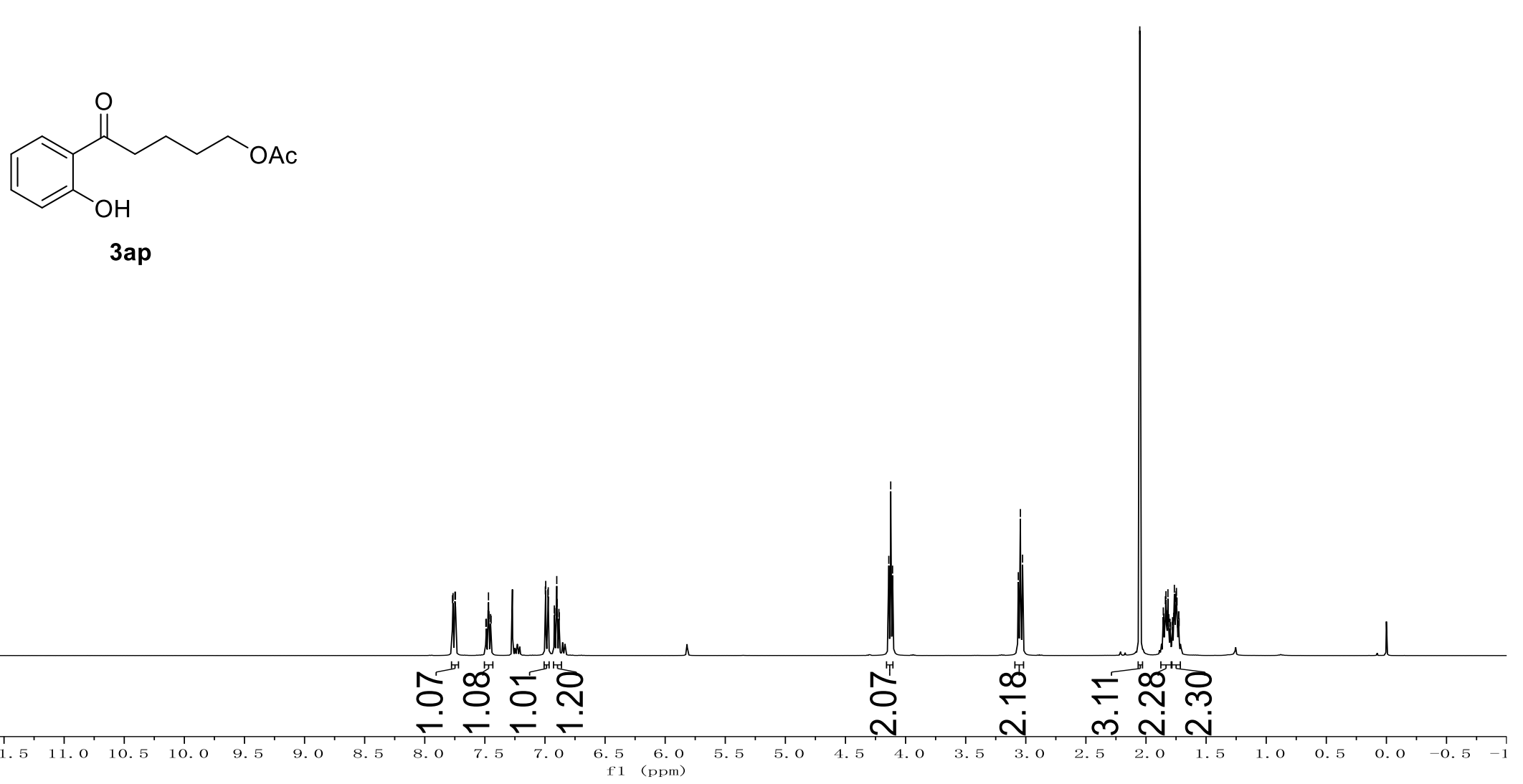
${ }^{13} \mathrm{C}$ NMR-spectrum $\left(101 \mathrm{MHz}, \mathrm{CDCl}_{3}\right)$ of $\mathbf{3 a p}$

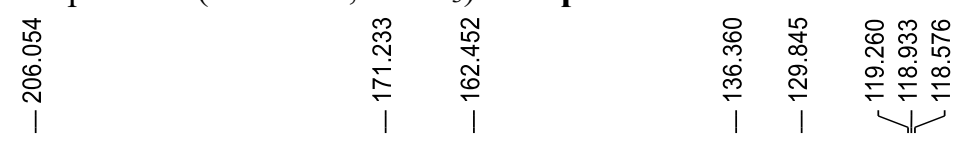
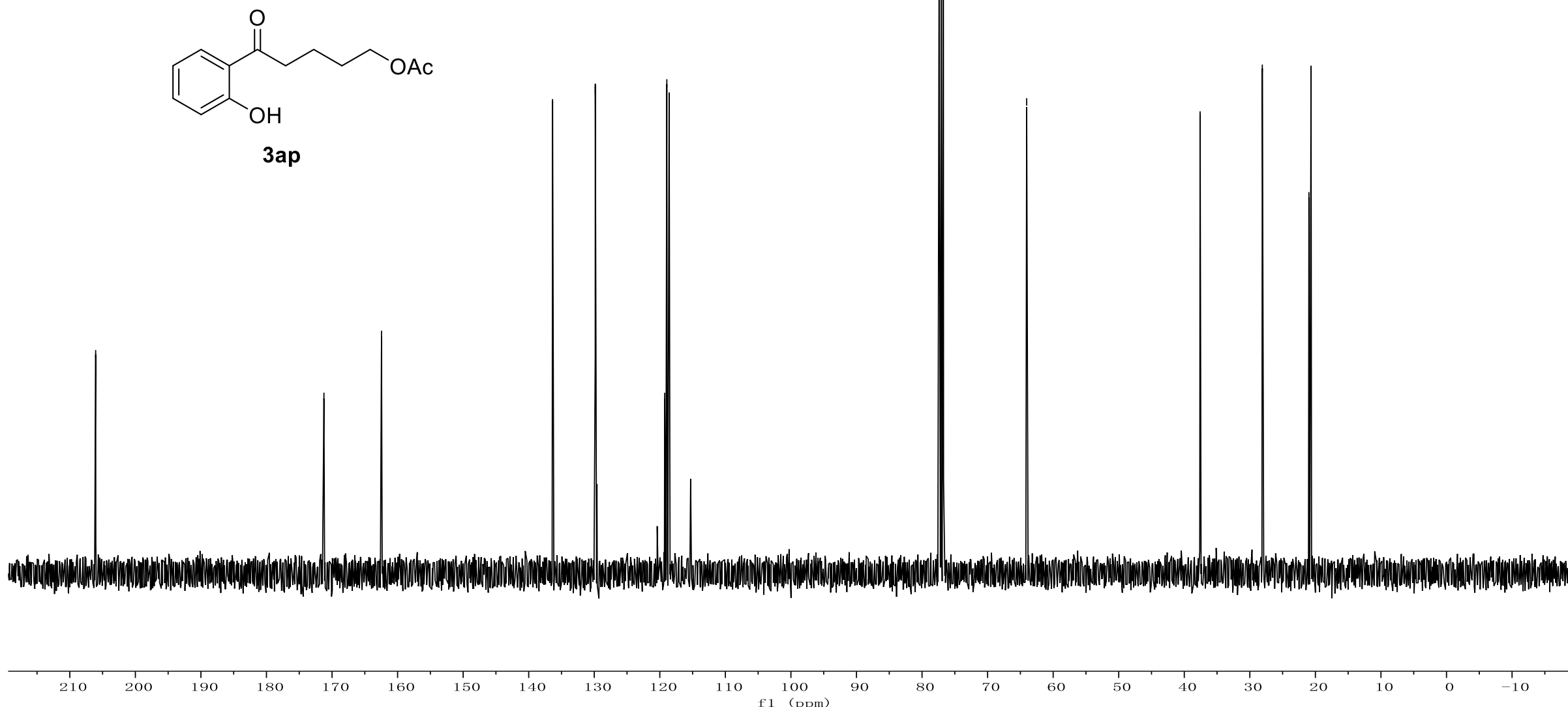
${ }^{1} \mathrm{H}$ NMR-spectrum $\left(400 \mathrm{MHz}, \mathrm{CDCl}_{3}\right)$ of $\mathbf{3 a q}$

$\stackrel{\substack{\infty \\ \stackrel{\sim}{\sim}}}{\sim}$

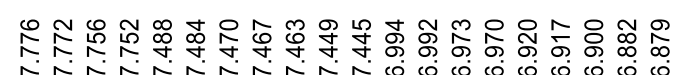

o 000000

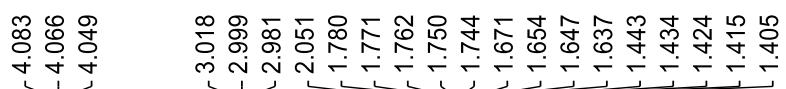
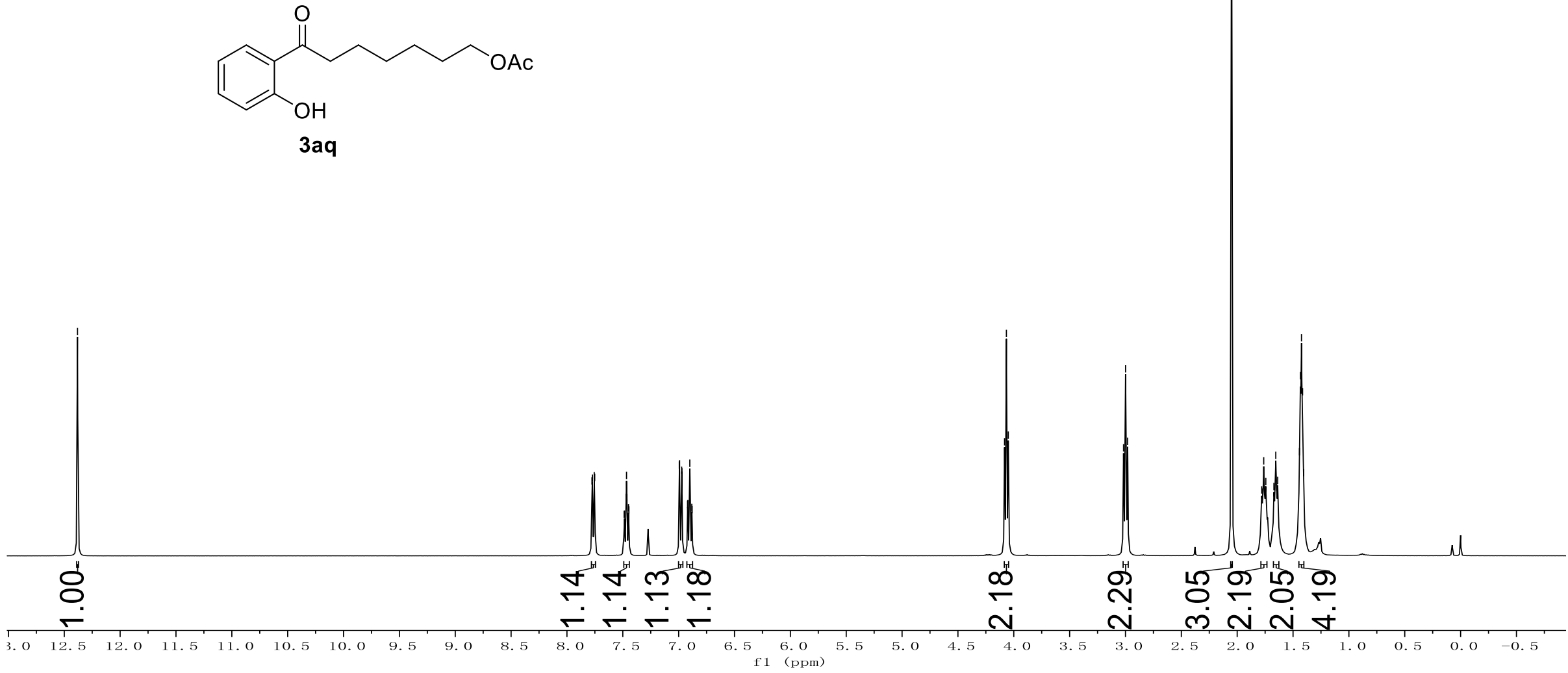
${ }^{13} \mathrm{C}$ NMR-spectrum $\left(101 \mathrm{MHz}, \mathrm{CDCl}_{3}\right)$ of $\mathbf{3 a q}$

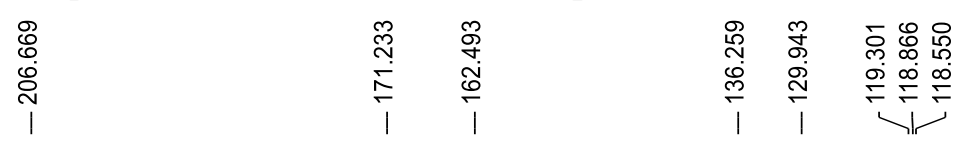

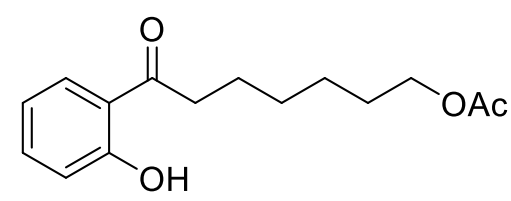

$3 \mathrm{aq}$

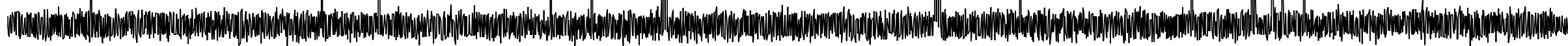

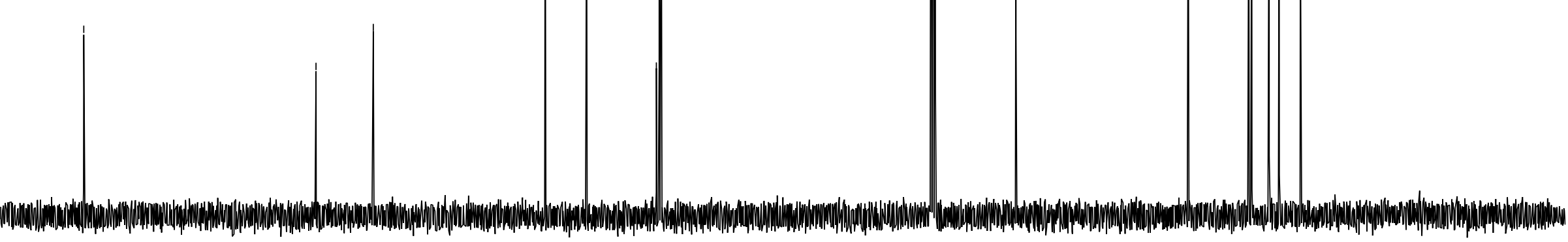


${ }^{1} \mathrm{H}$ NMR-spectrum $\left(400 \mathrm{MHz}, \mathrm{CDCl}_{3}\right)$ of $\mathbf{3 a r}$

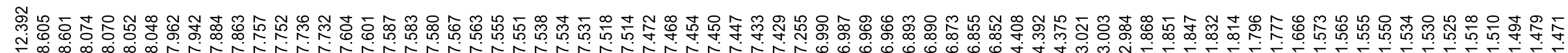

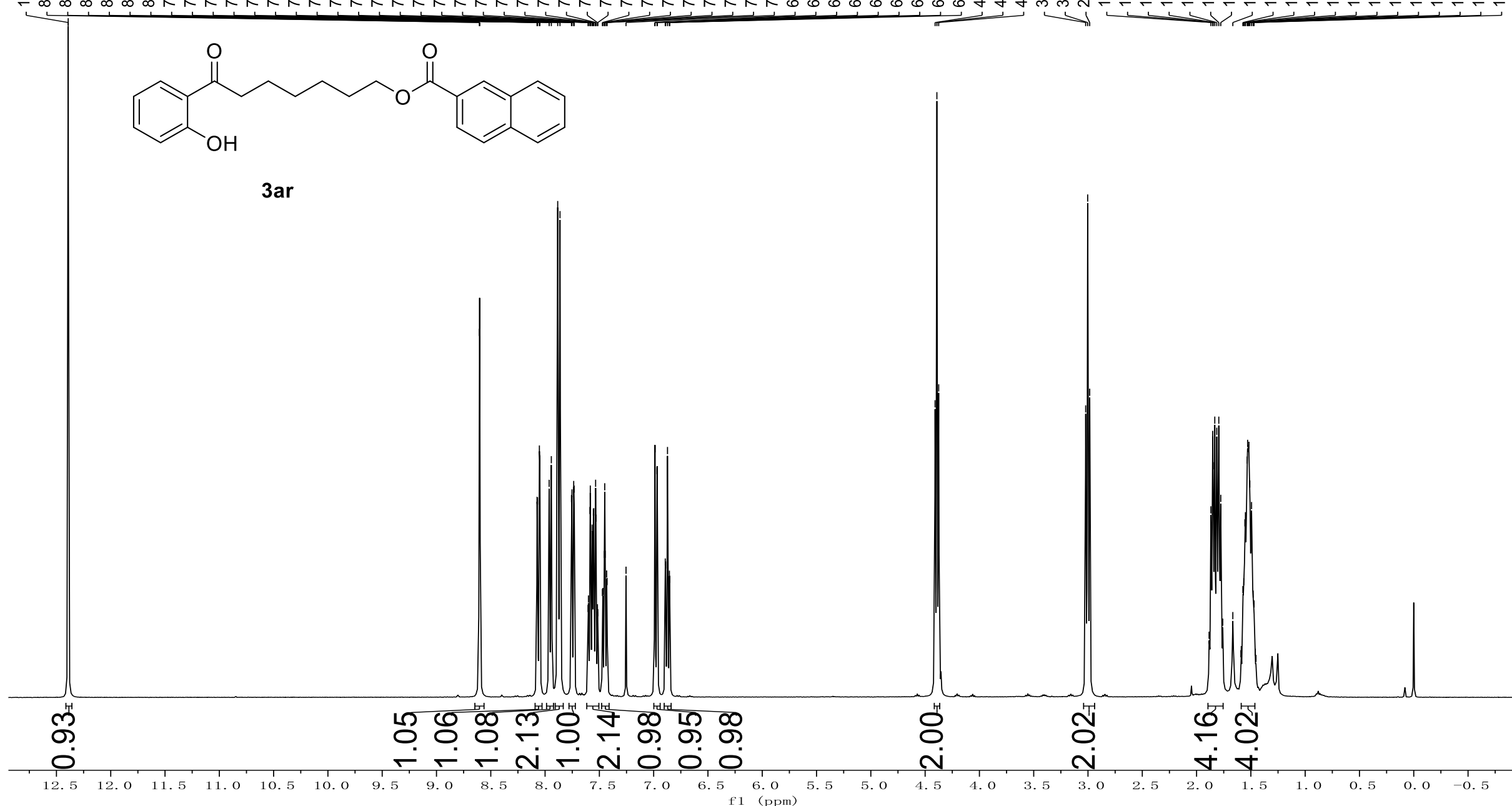


${ }^{13} \mathrm{C}$ NMR-spectrum $\left(101 \mathrm{MHz}, \mathrm{CDCl}_{3}\right)$ of $\mathbf{3 a r}$

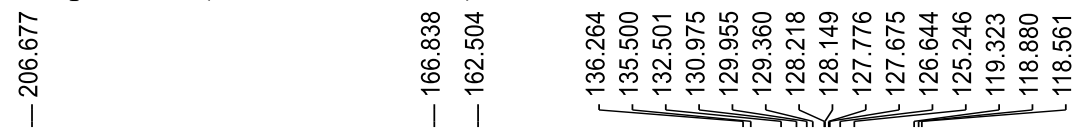

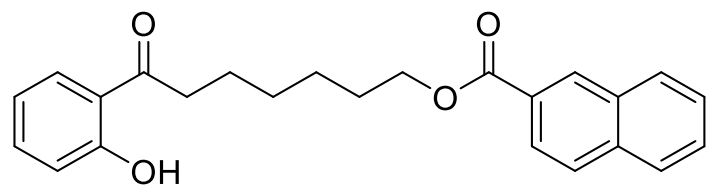

$3 a r$
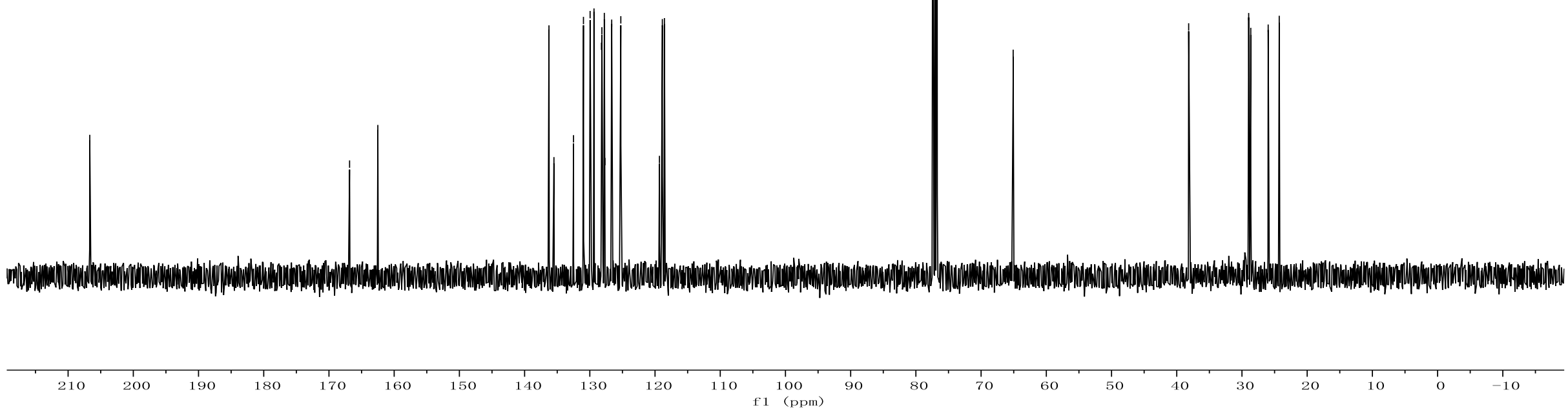

S103 
${ }^{1} \mathrm{H}$ NMR-spectrum $\left(500 \mathrm{MHz}, \mathrm{CDCl}_{3}\right)$ of 3as

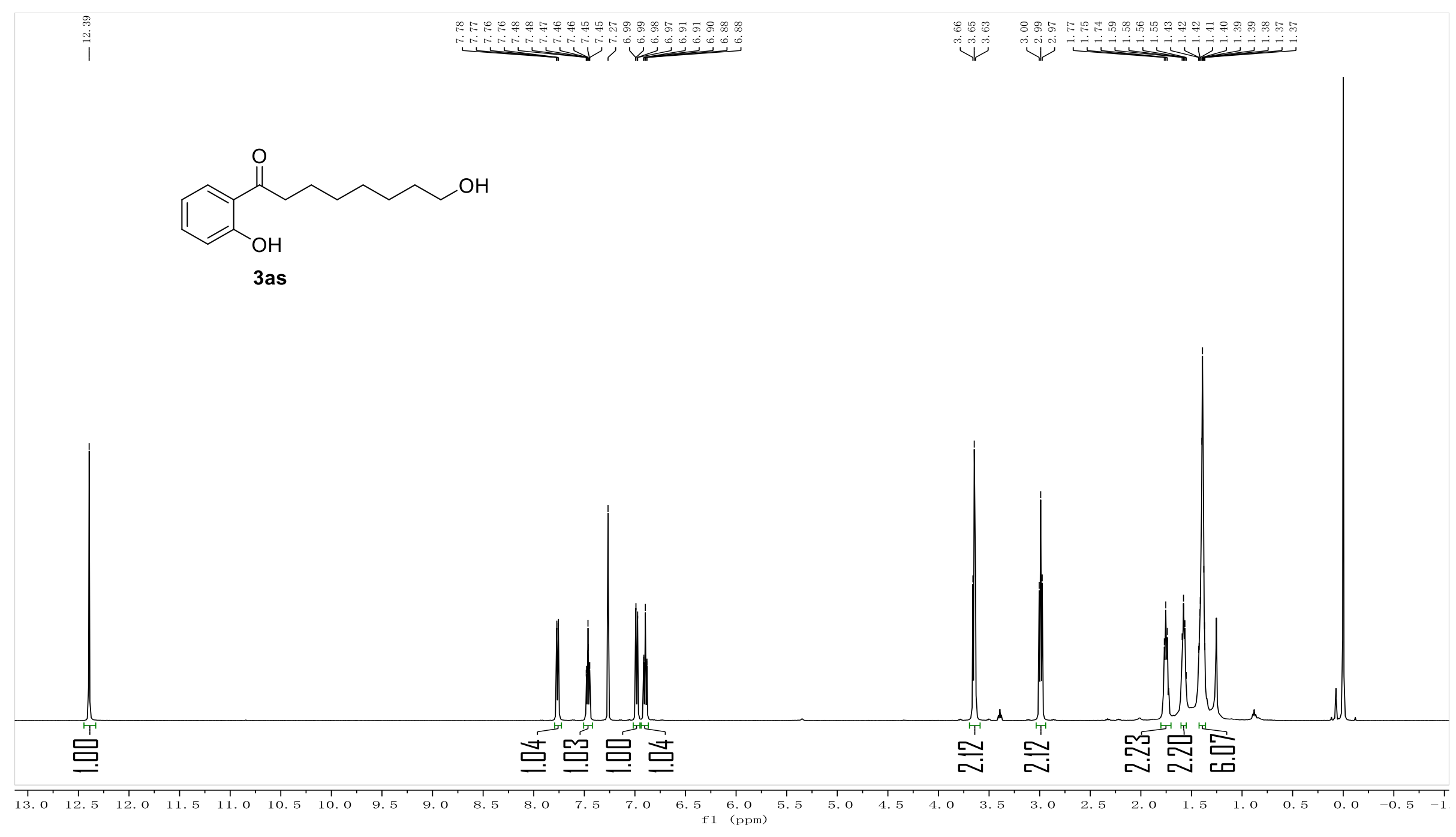


${ }^{13} \mathrm{C}$ NMR-spectrum $\left(126 \mathrm{MHz}, \mathrm{CDCl}_{3}\right)$ of $\mathbf{3 a s}$

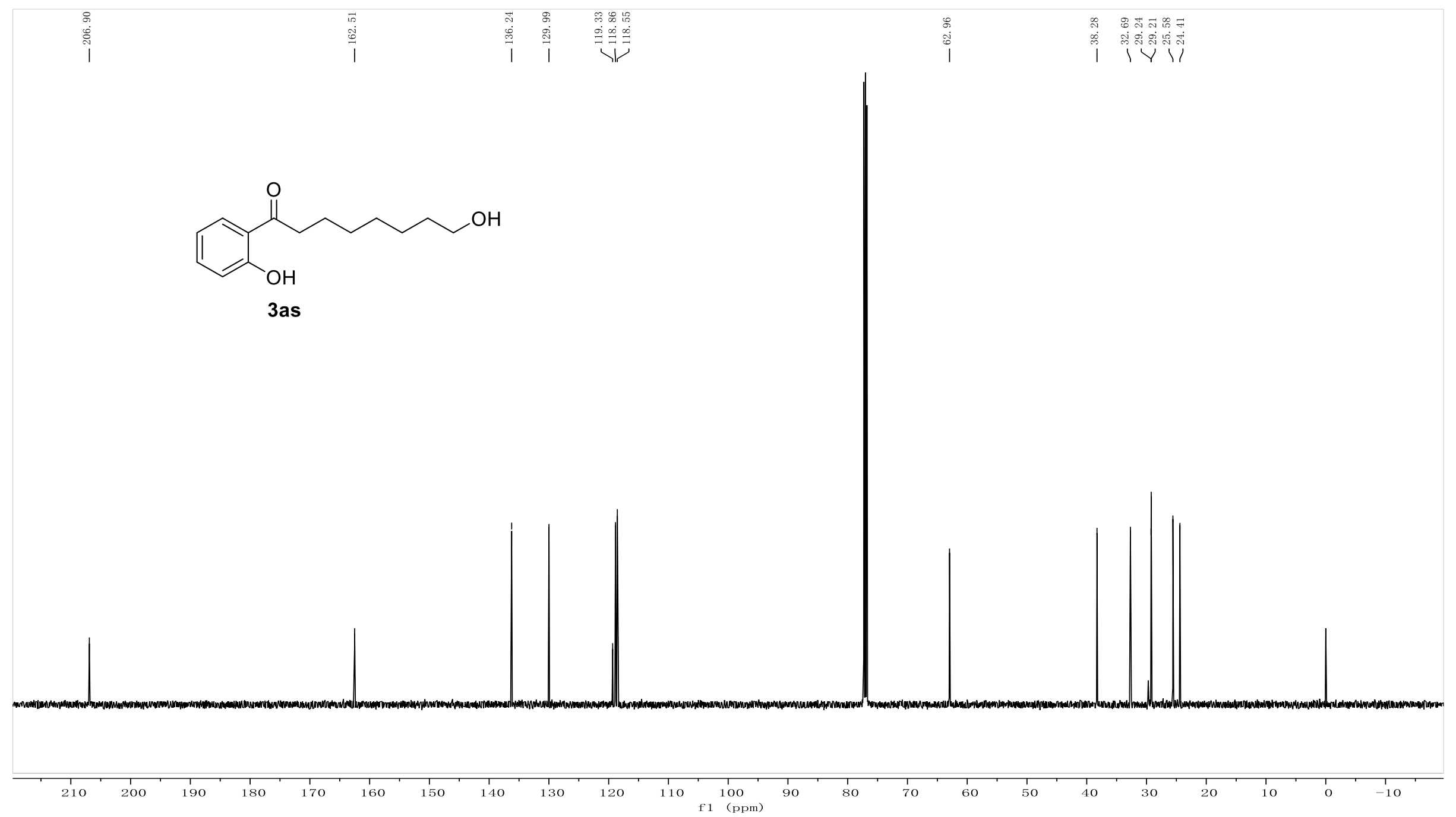


${ }^{1} \mathrm{H}$ NMR-spectrum $\left(400 \mathrm{MHz}, \mathrm{CDCl}_{3}\right)$ of 3at

$\stackrel{\text { }}{\stackrel{0}{\sim}}$

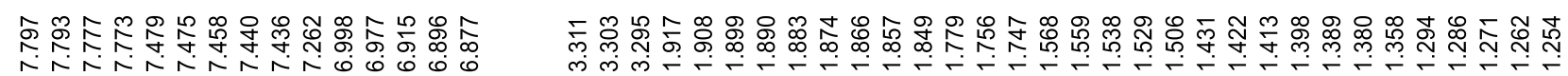
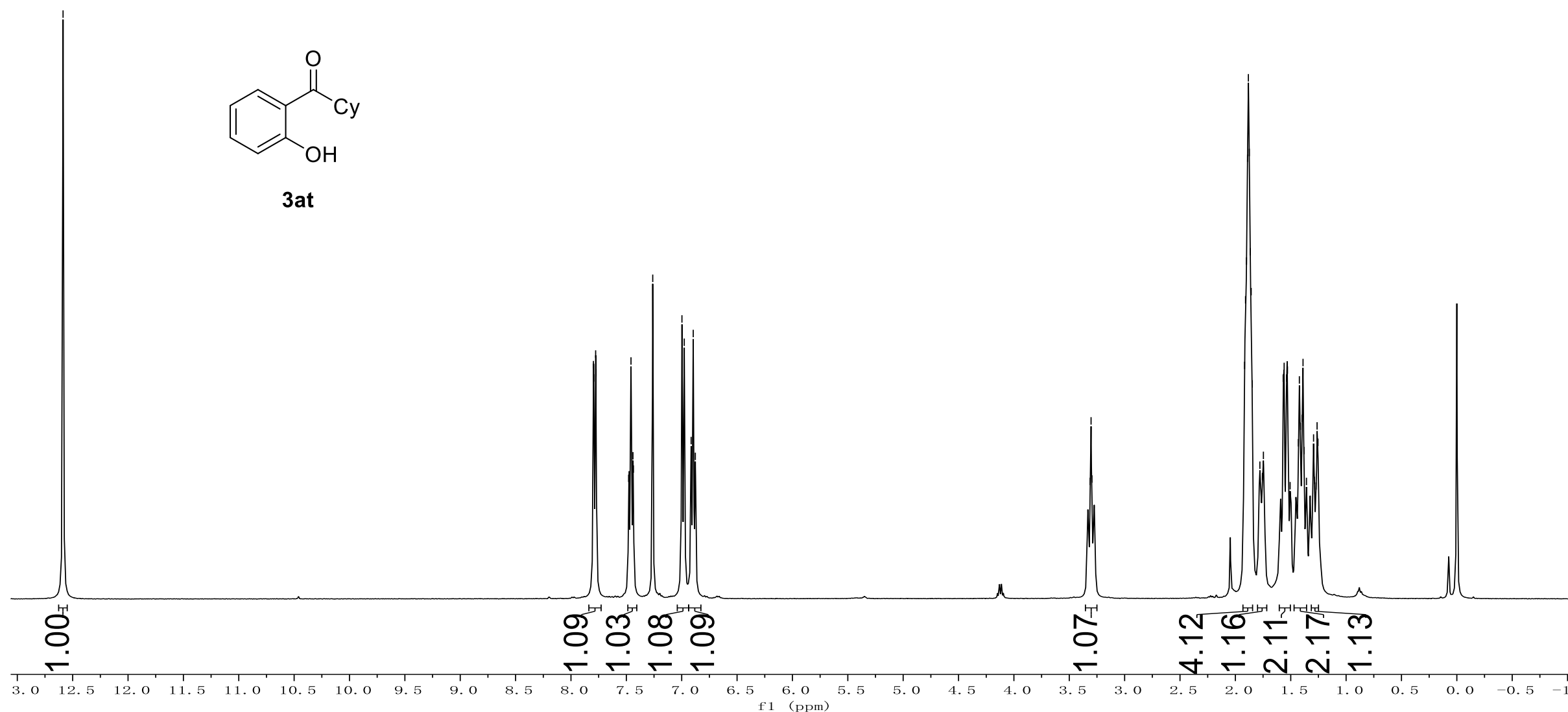
${ }^{13} \mathrm{C}$ NMR-spectrum $\left(101 \mathrm{MHz}, \mathrm{CDCl}_{3}\right)$ of 3 at

융

胥

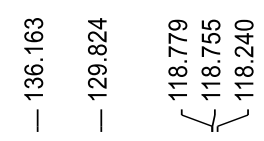

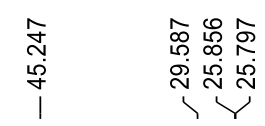

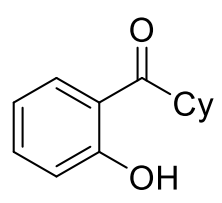

3at

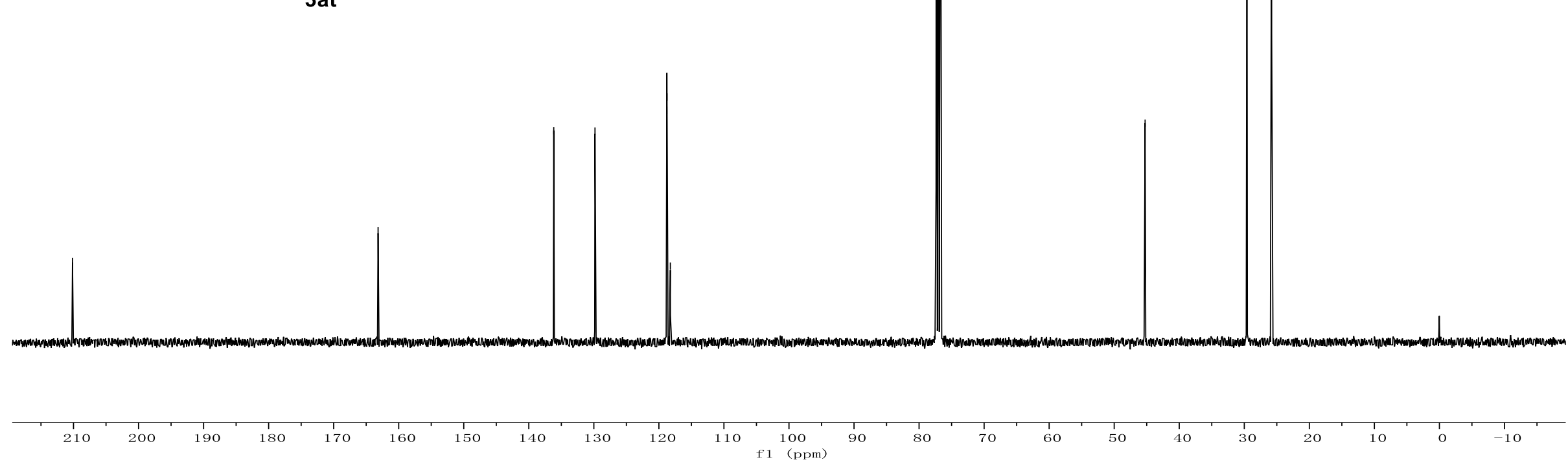

S107 
${ }^{1} \mathrm{H}$ NMR-spectrum $\left(500 \mathrm{MHz}, \mathrm{CDCl}_{3}\right)$ of $\mathbf{3 a u}$

广্ণ

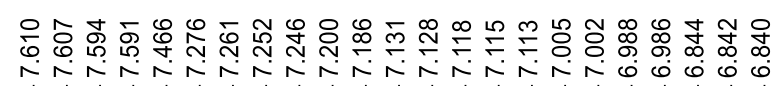

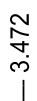

$\underbrace{.0050}$

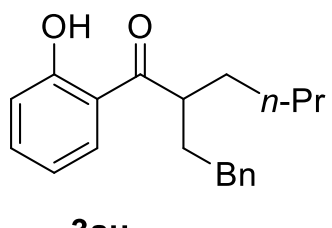

$3 a u$
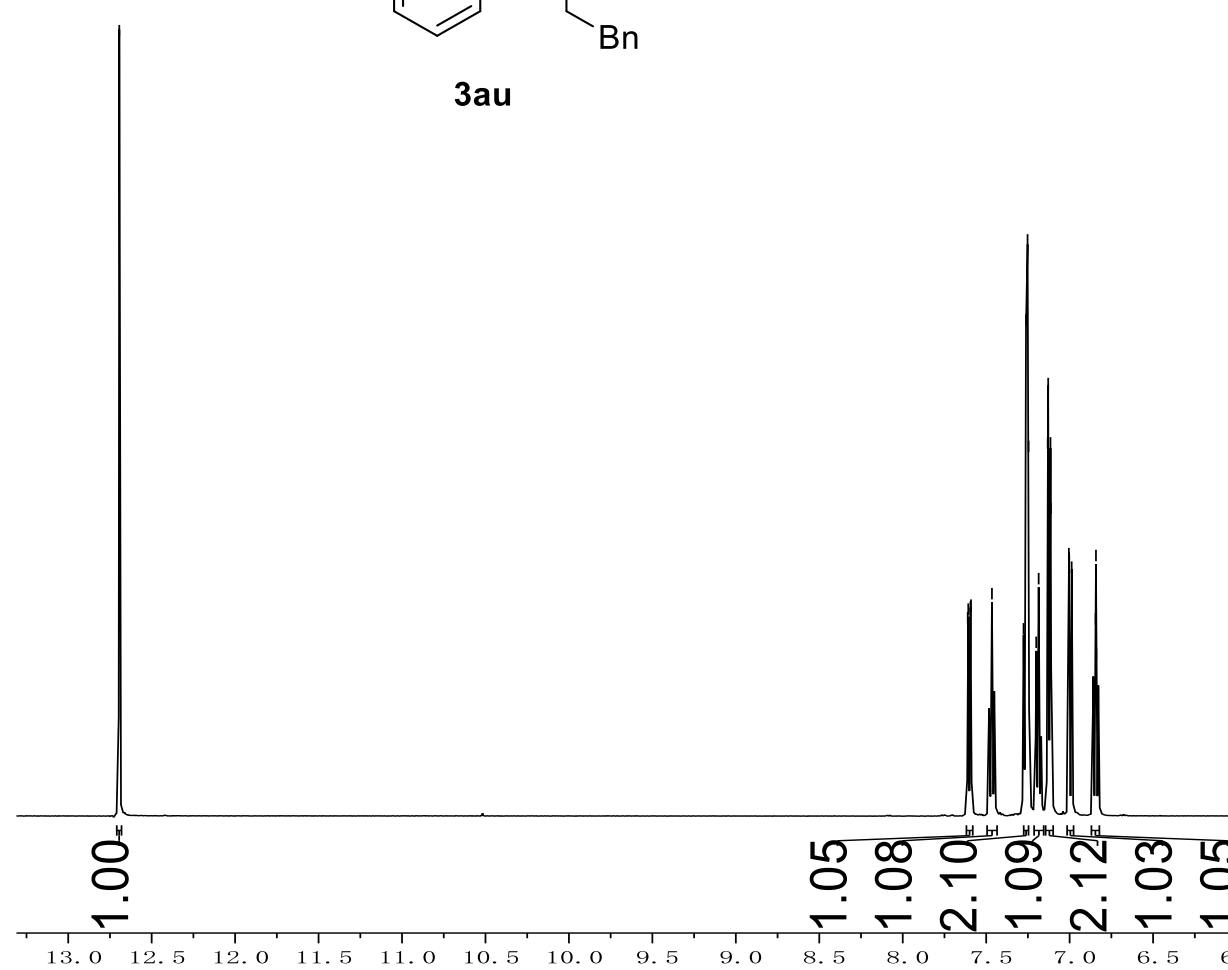

f1 (ppm) 
${ }^{13} \mathrm{C}$ NMR-spectrum $\left(101 \mathrm{MHz}, \mathrm{CDCl}_{3}\right)$ of $\mathbf{3 a u}$
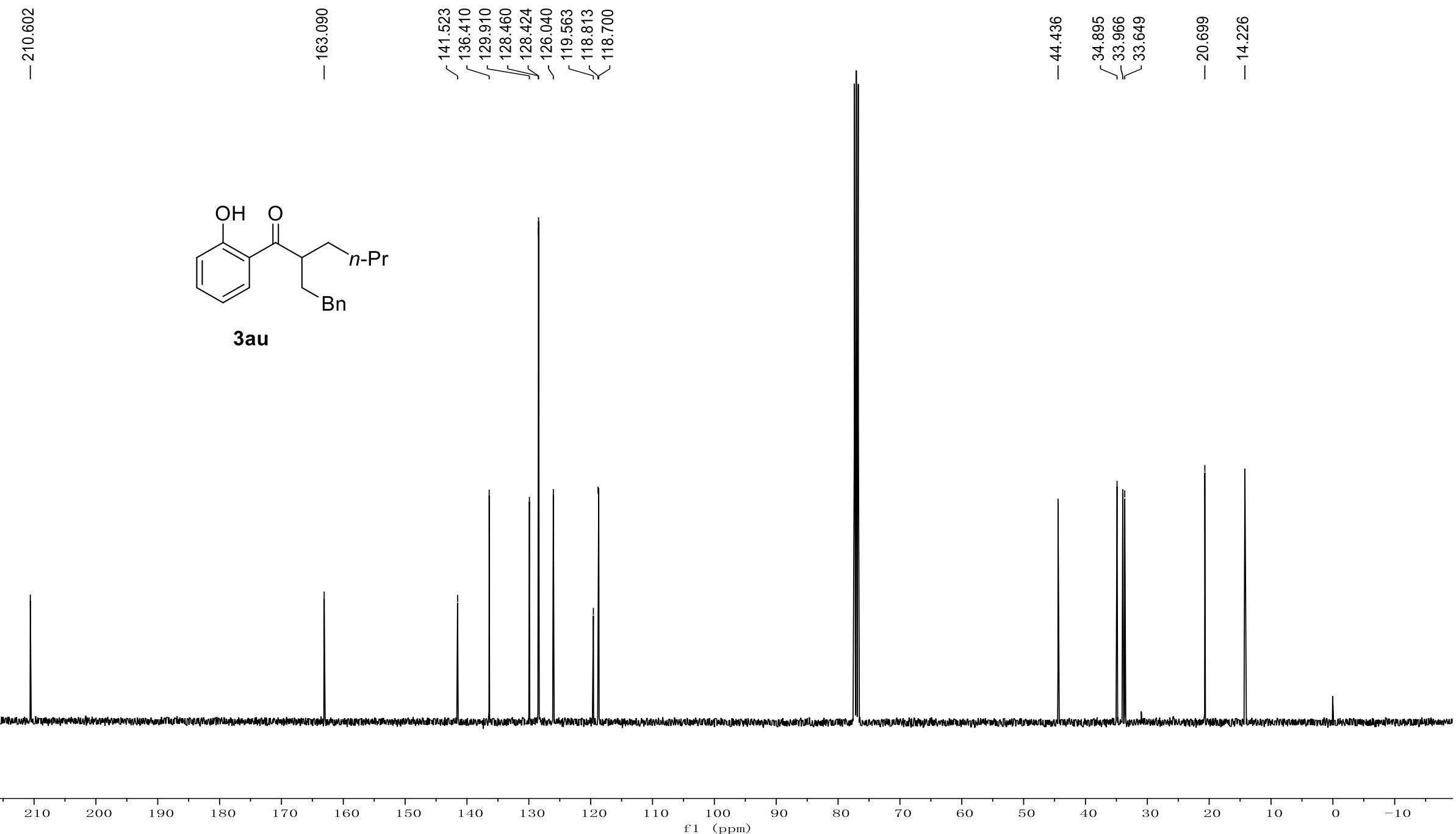
${ }^{1} \mathrm{H}$ NMR-spectrum $\left(500 \mathrm{MHz}, \mathrm{CDCl}_{3}\right)$ of $\mathbf{3 a v}$

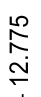

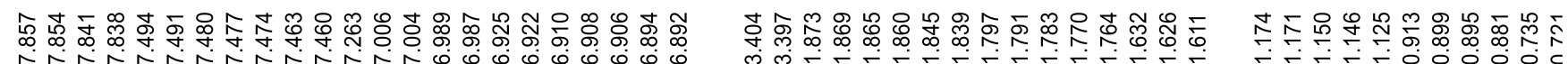
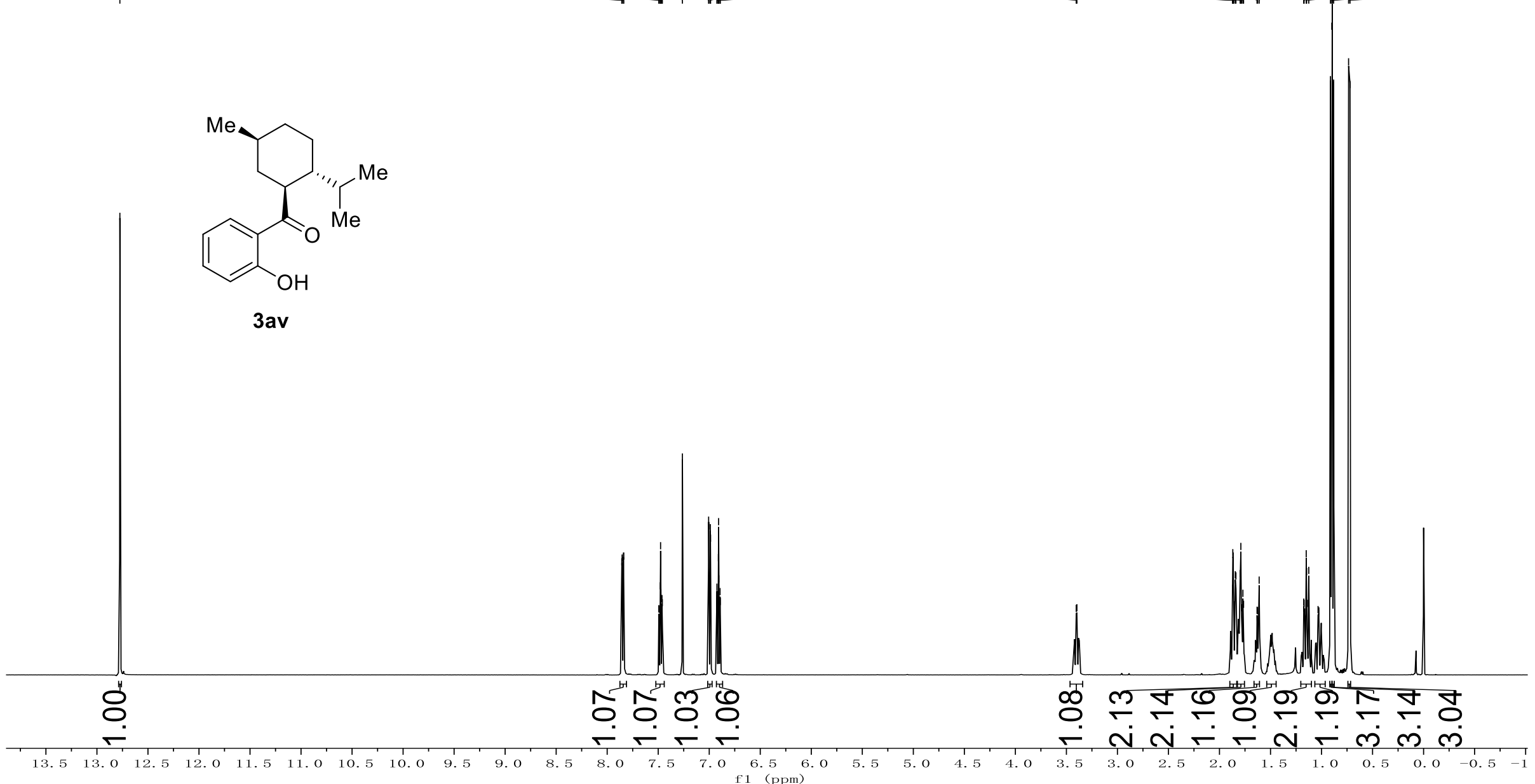
${ }^{13} \mathrm{C}$ NMR-spectrum $\left(101 \mathrm{MHz}, \mathrm{CDCl}_{3}\right)$ of $\mathbf{3 a v}$
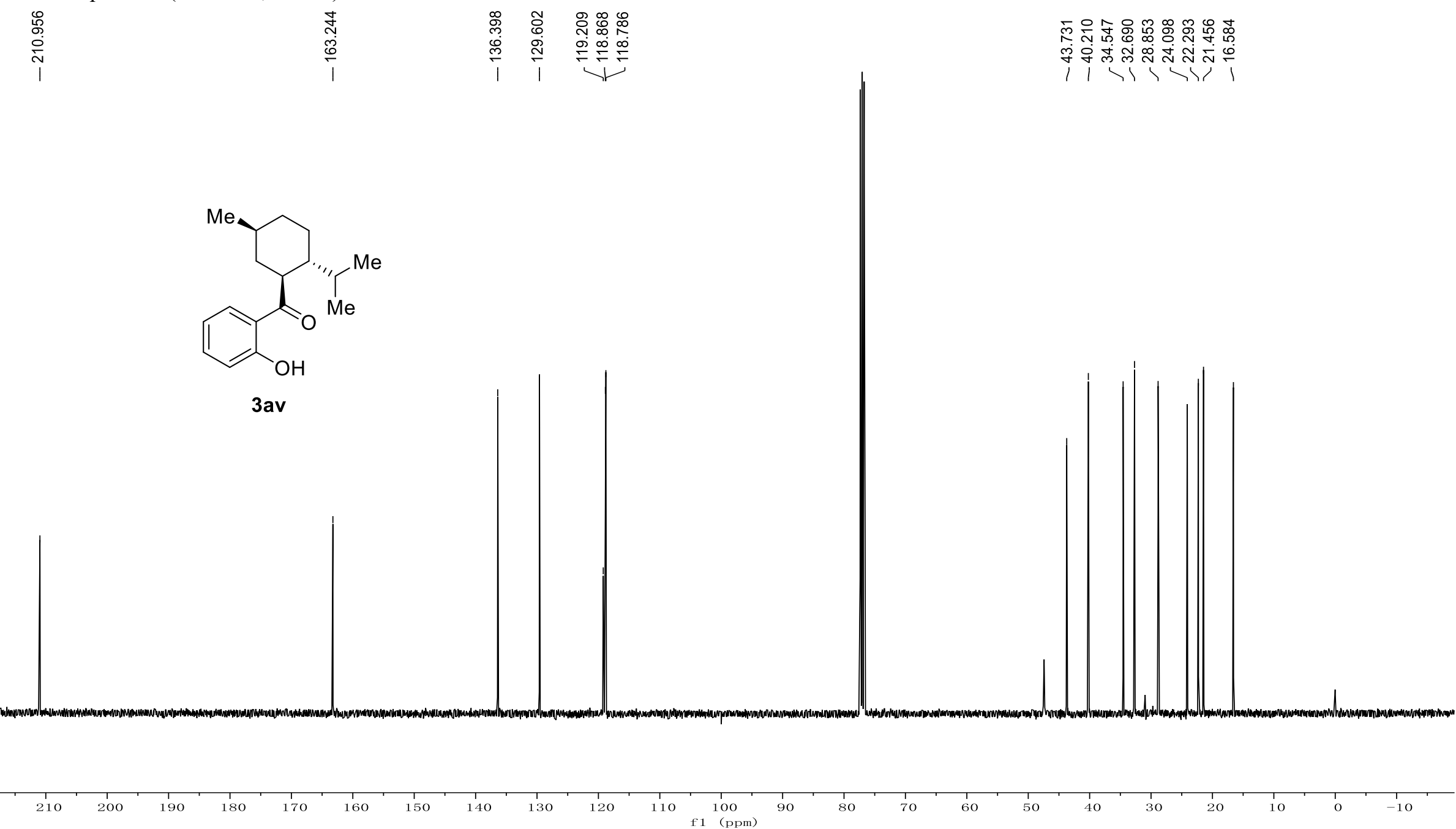
COSY-spectrum of $\mathbf{3 a v}$
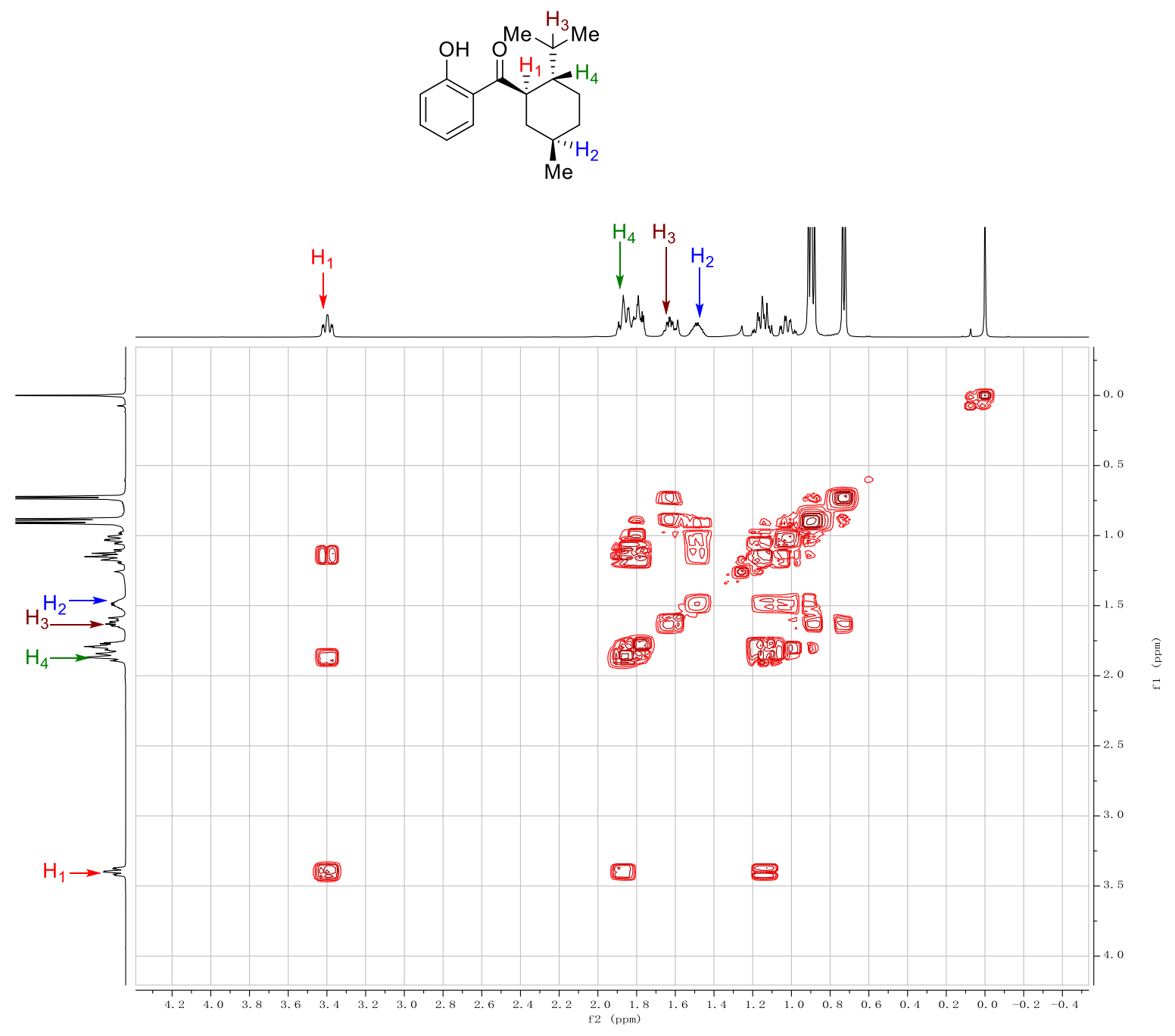
DEPT-135 ${ }^{\circ}$-spectrum of $\mathbf{3 a v}$
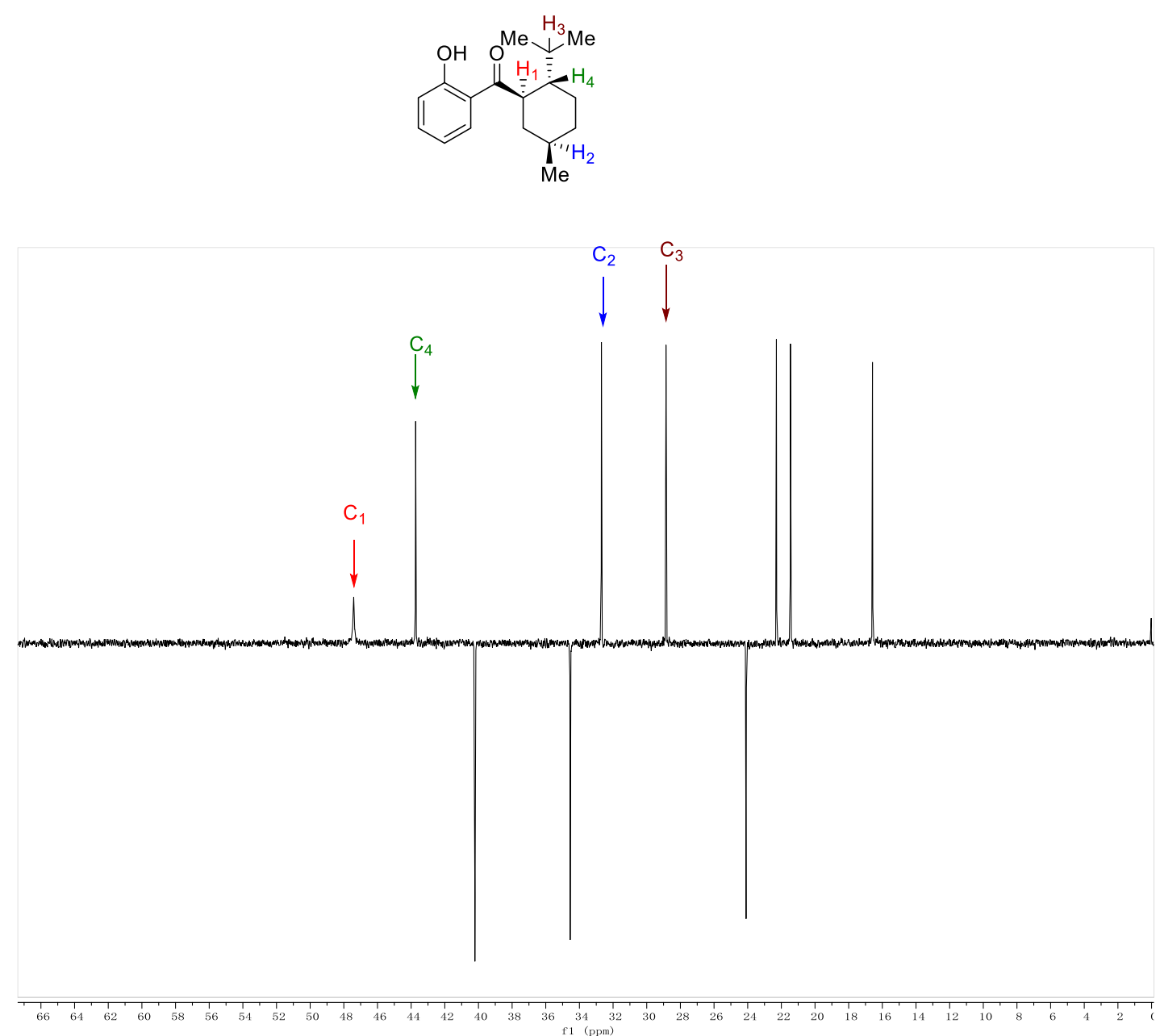
HSQC-spectrum of $\mathbf{3 a v}$

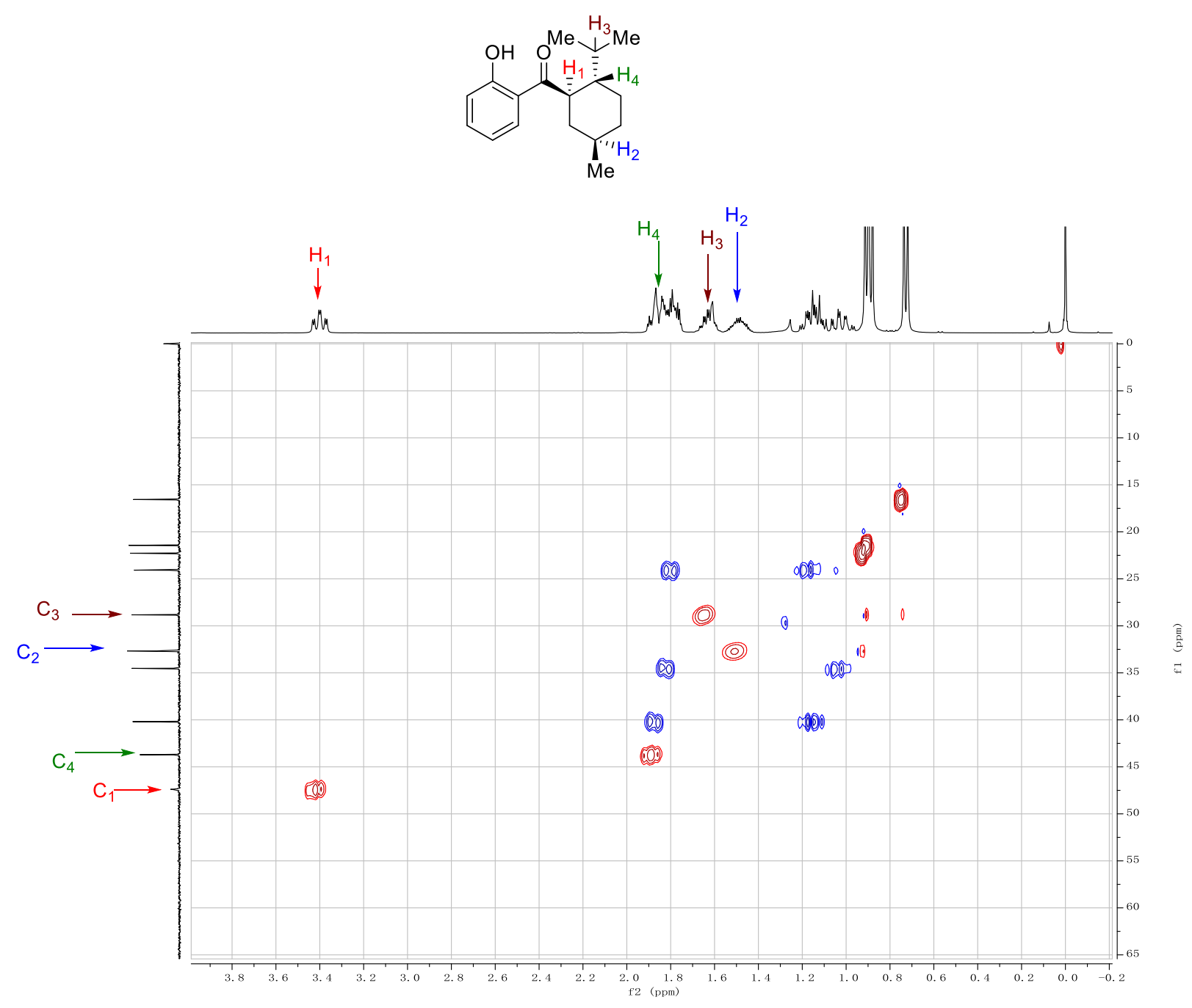


NOSEY-spectrum of $\mathbf{3 a v}$
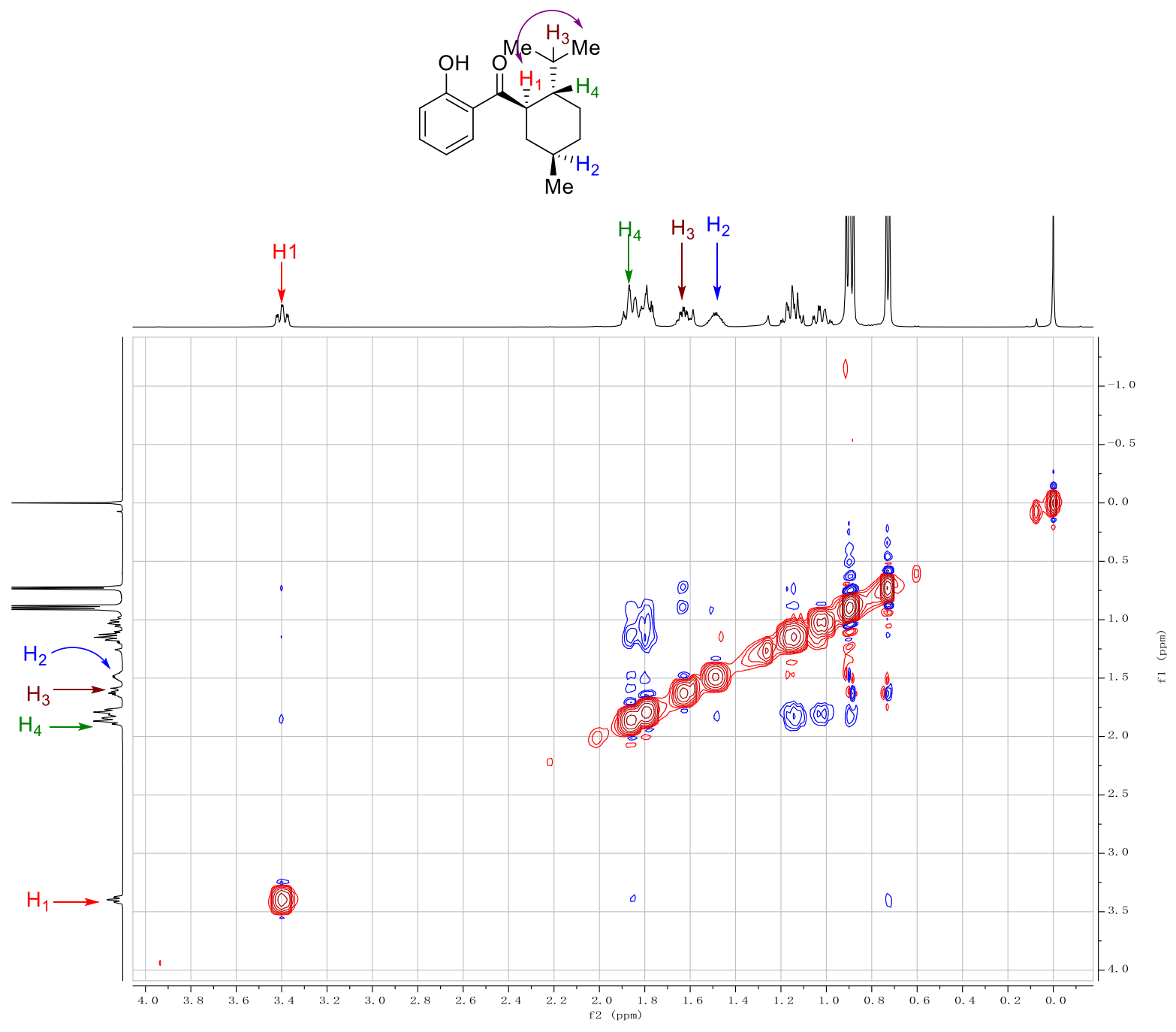

S115 
${ }^{1} \mathrm{H}$ NMR-spectrum $\left(500 \mathrm{MHz}, \mathrm{CDCl}_{3}\right)$ of 3aw and 3aw'

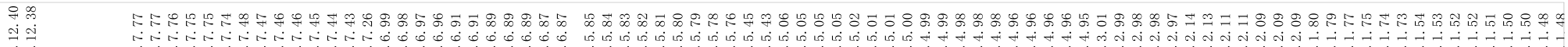
仙

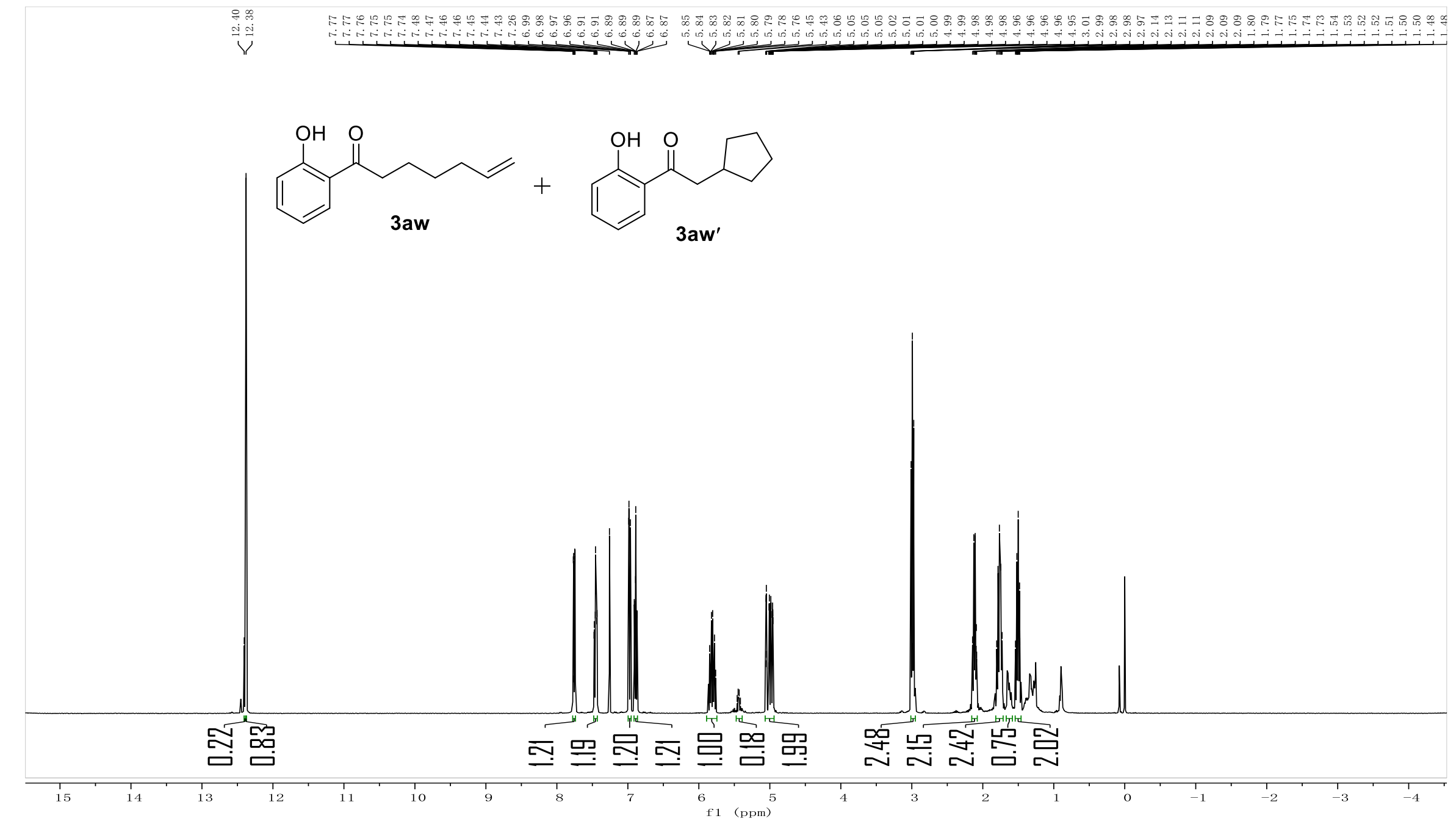


${ }^{1} \mathrm{H}$ NMR-spectrum $\left(400 \mathrm{MHz}, \mathrm{CDCl}_{3}\right)$ of $\mathbf{3} \mathbf{b k}$

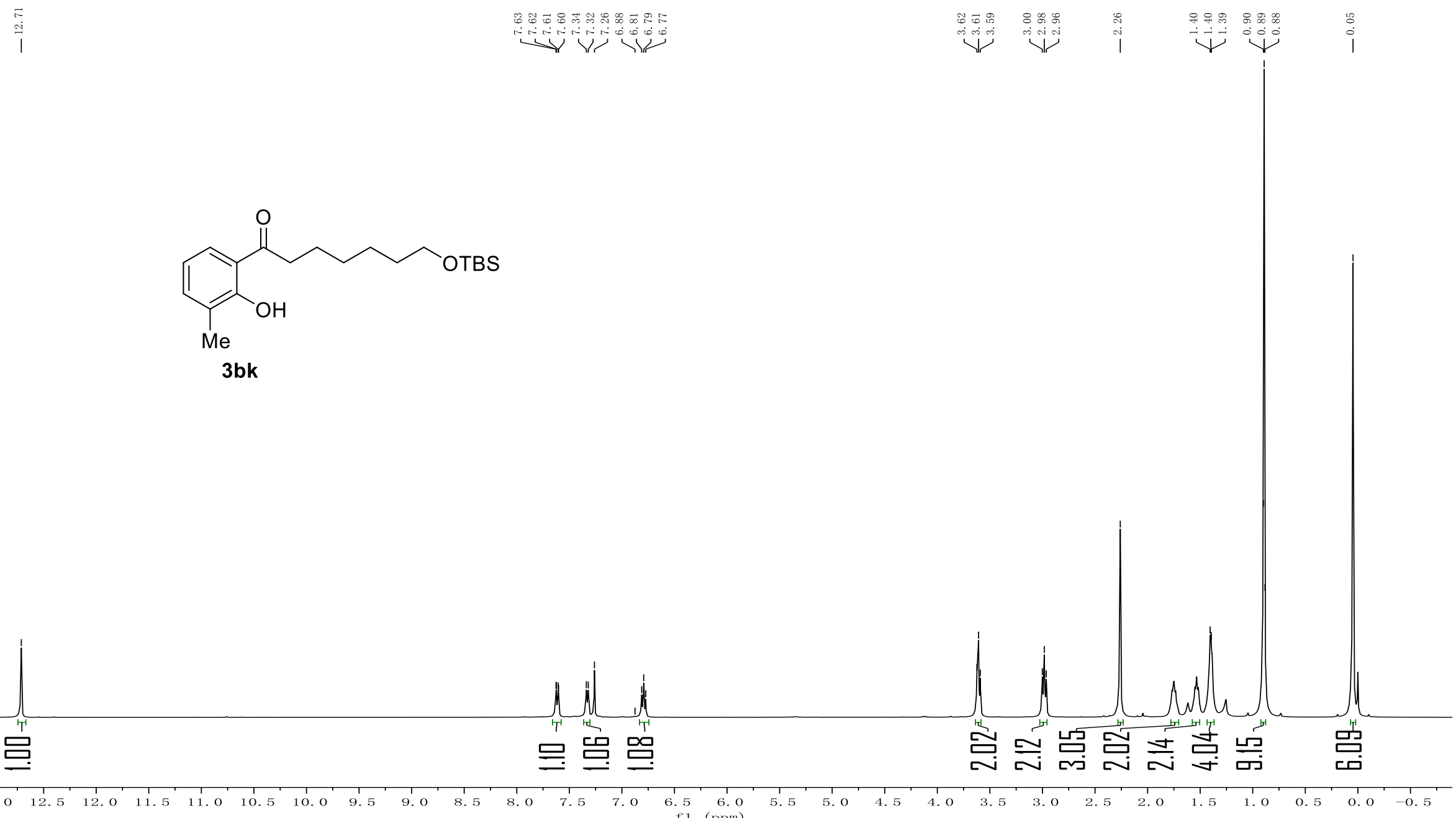


${ }^{13} \mathrm{C} \mathrm{NMR-spectrum}\left(101 \mathrm{MHz}, \mathrm{CDCl}_{3}\right)$ of $\mathbf{3 b k}$
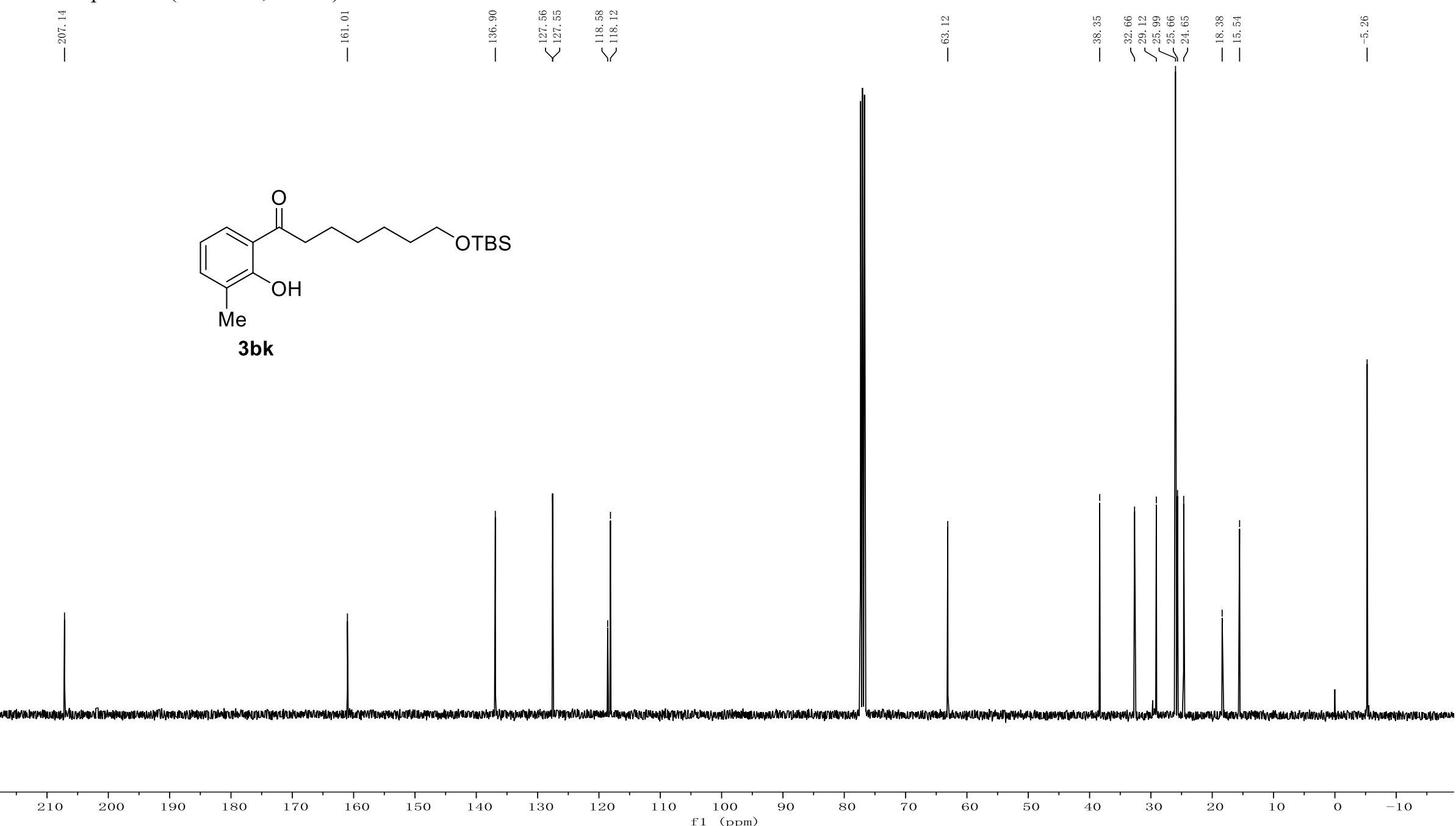
${ }^{1} \mathrm{H}$ NMR-spectrum $\left(400 \mathrm{MHz}, \mathrm{CDCl}_{3}\right.$ ) of 3ck

$\stackrel{\substack{3 \\ \text { I }}}{1}$

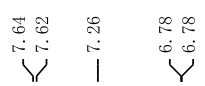

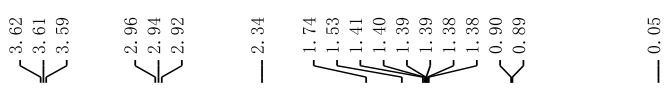

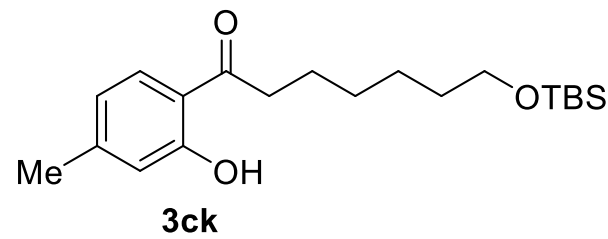

ck

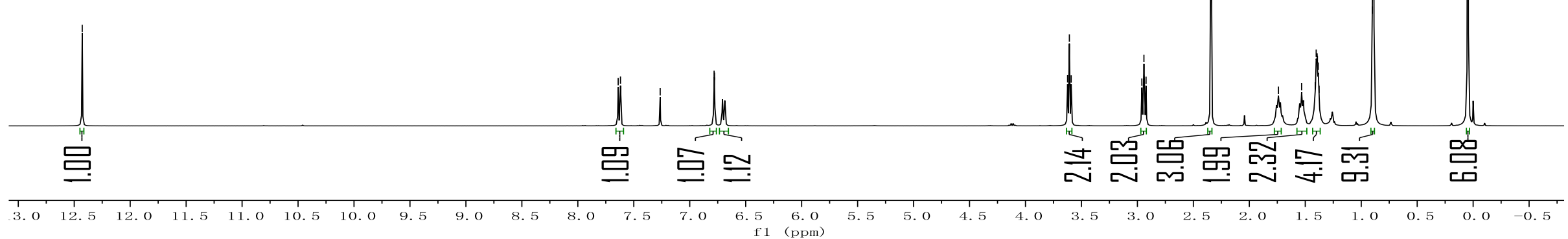


${ }^{13} \mathrm{C}$ NMR-spectrum $\left(101 \mathrm{MHz}, \mathrm{CDCl}_{3}\right)$ of 3ck
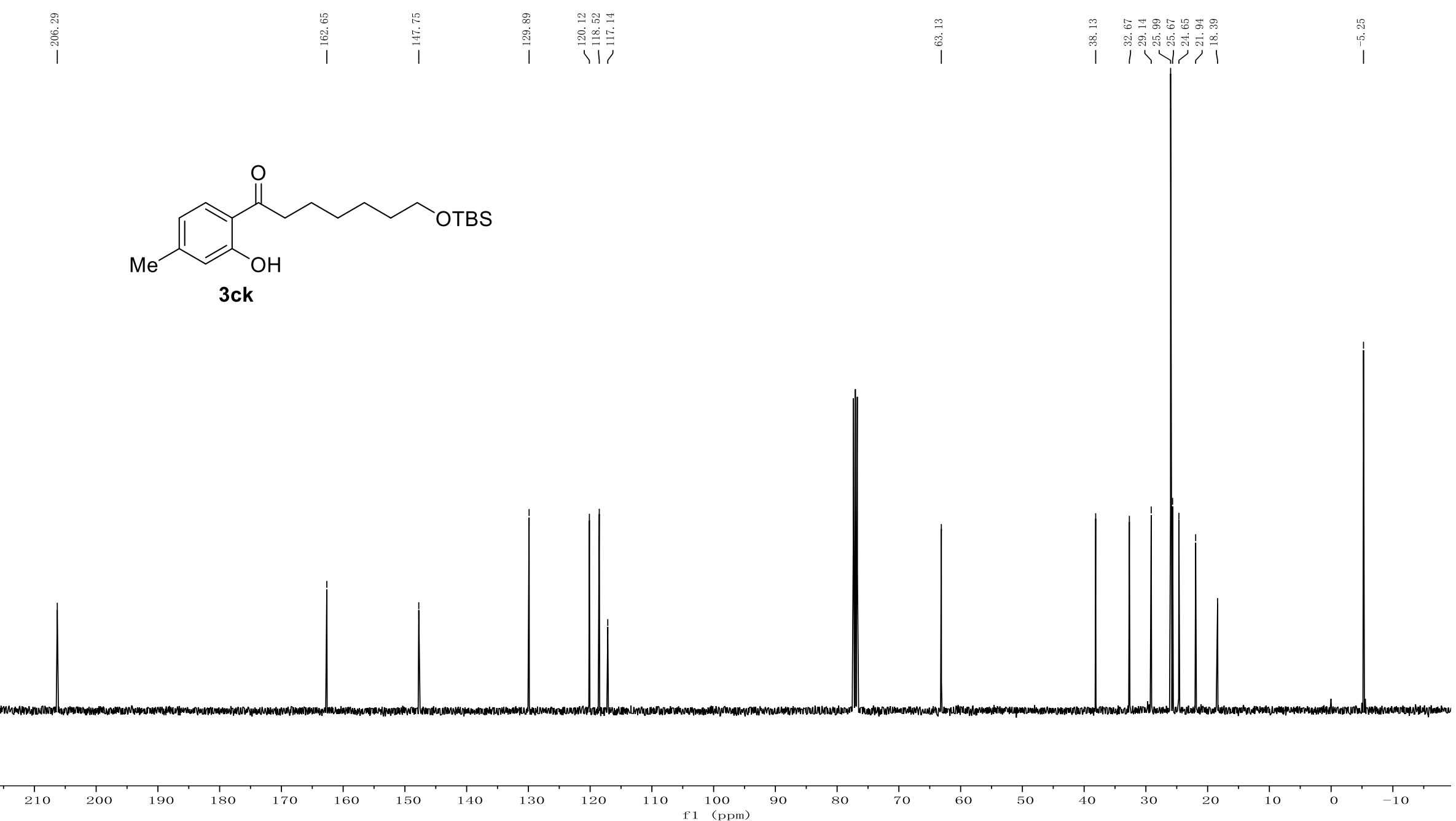
${ }^{1} \mathrm{H}$ NMR-spectrum $\left(400 \mathrm{MHz}, \mathrm{CDCl}_{3}\right)$ of $\mathbf{3 d k}$

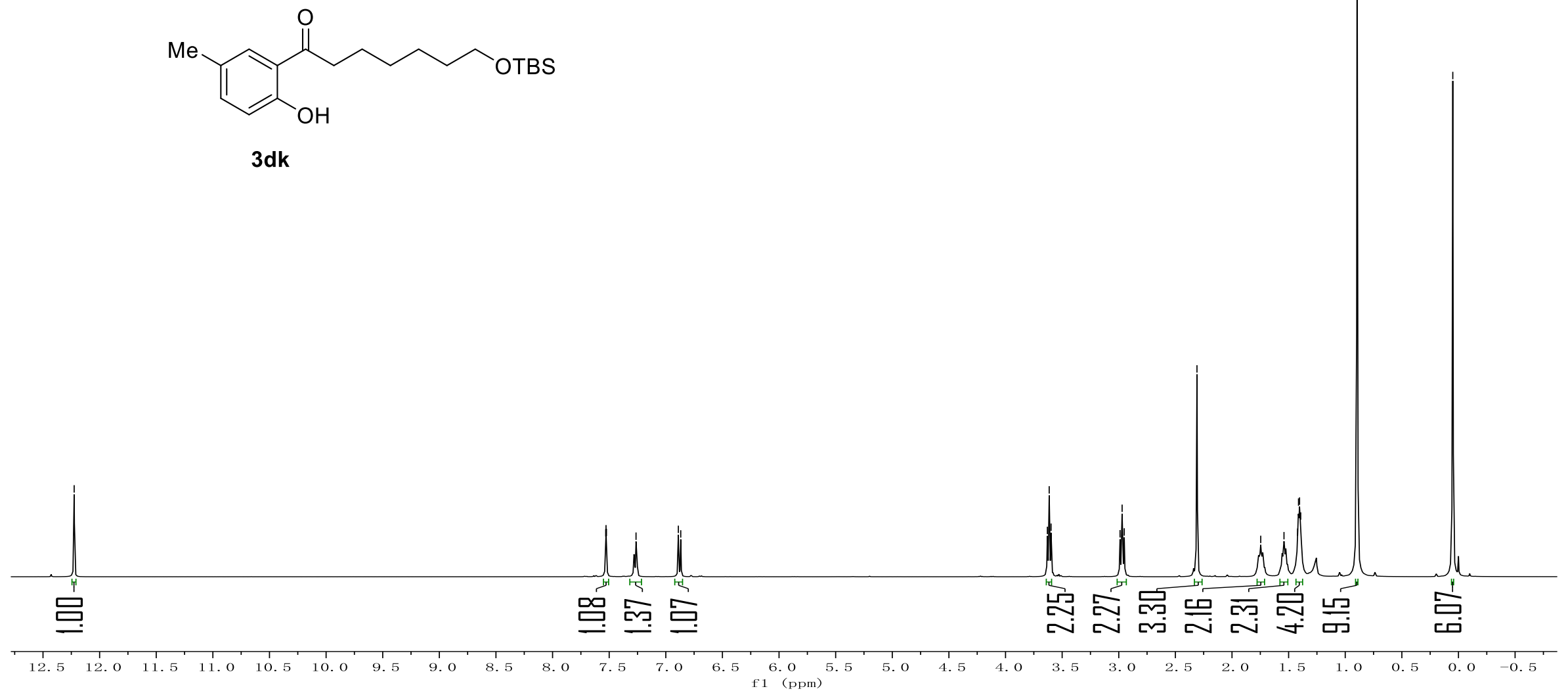

$3 \mathrm{dk}$ 
${ }^{13} \mathrm{C}$ NMR-spectrum $\left(101 \mathrm{MHz}, \mathrm{CDCl}_{3}\right)$ of $\mathbf{3 d k}$

每

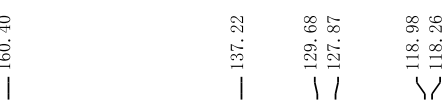

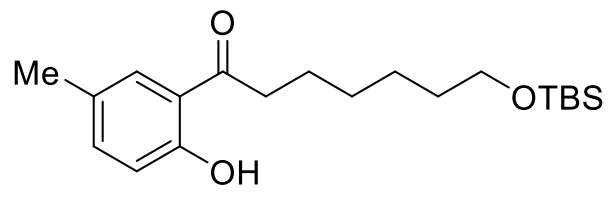

3dk
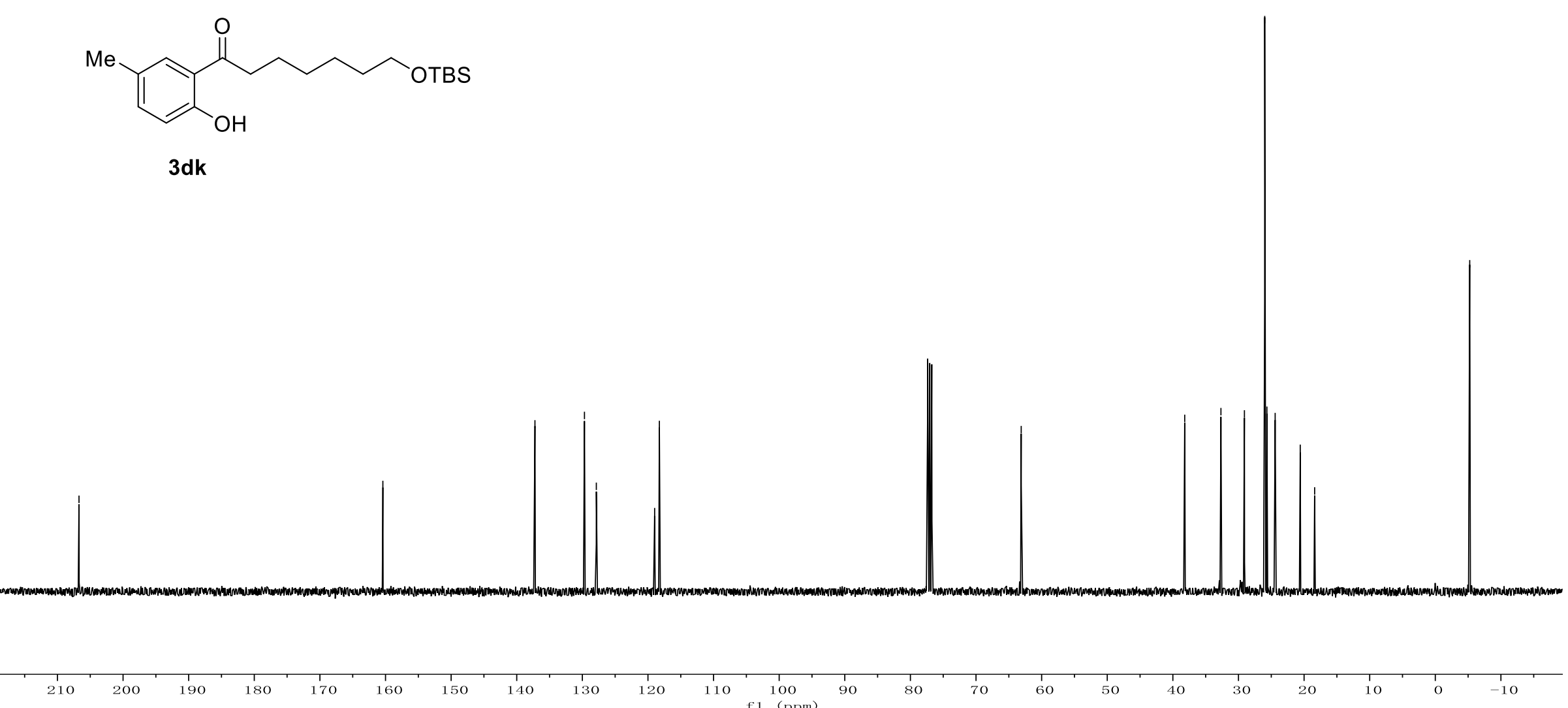
${ }^{1} \mathrm{H}$ NMR-spectrum $\left(400 \mathrm{MHz}, \mathrm{CDCl}_{3}\right)$ of $\mathbf{3 e k}$

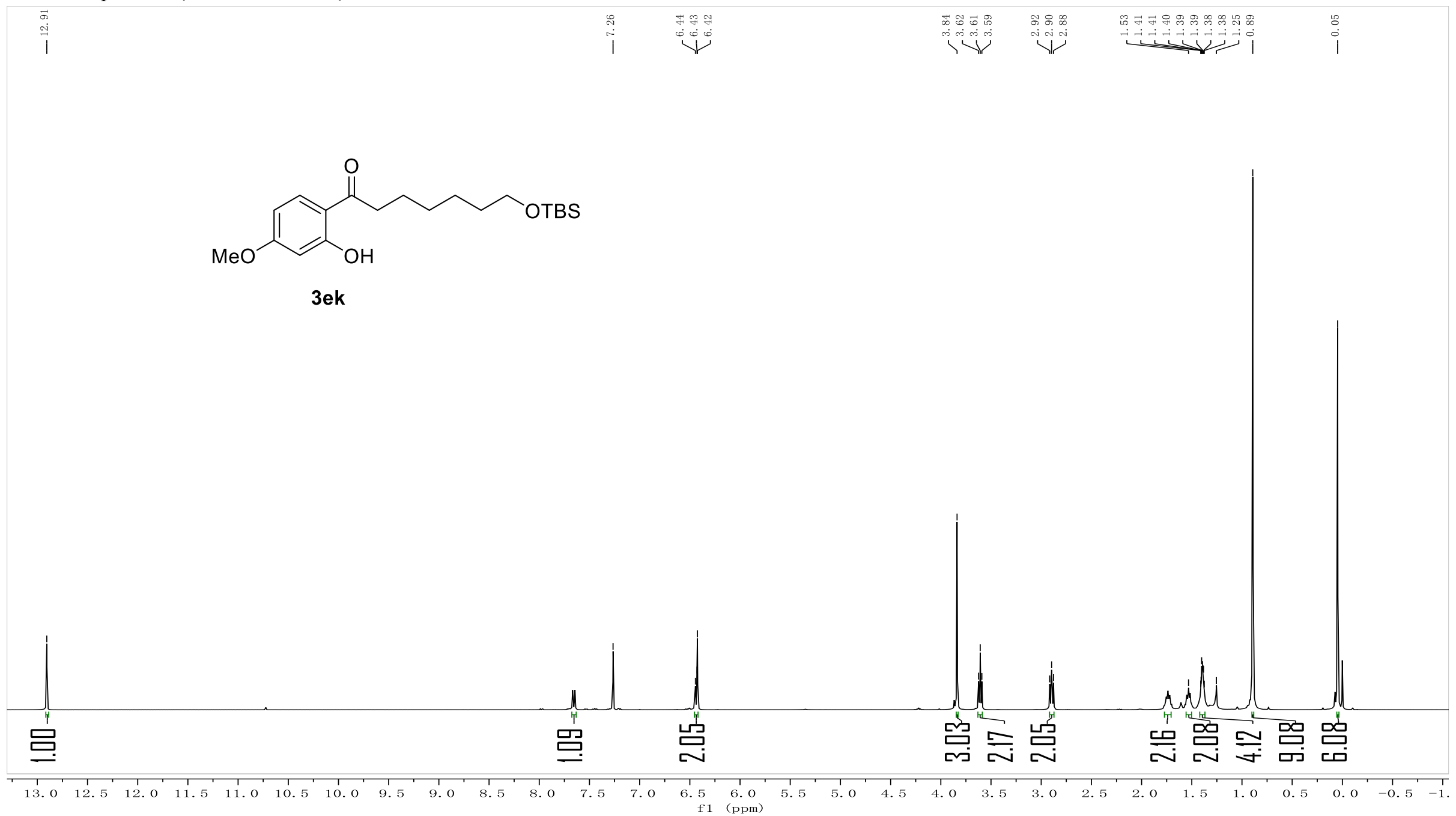


${ }^{13} \mathrm{C}$ NMR-spectrum $\left(101 \mathrm{MHz}, \mathrm{CDCl}_{3}\right)$ of $\mathbf{3 e k}$

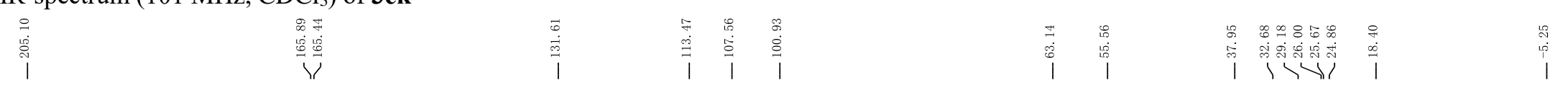
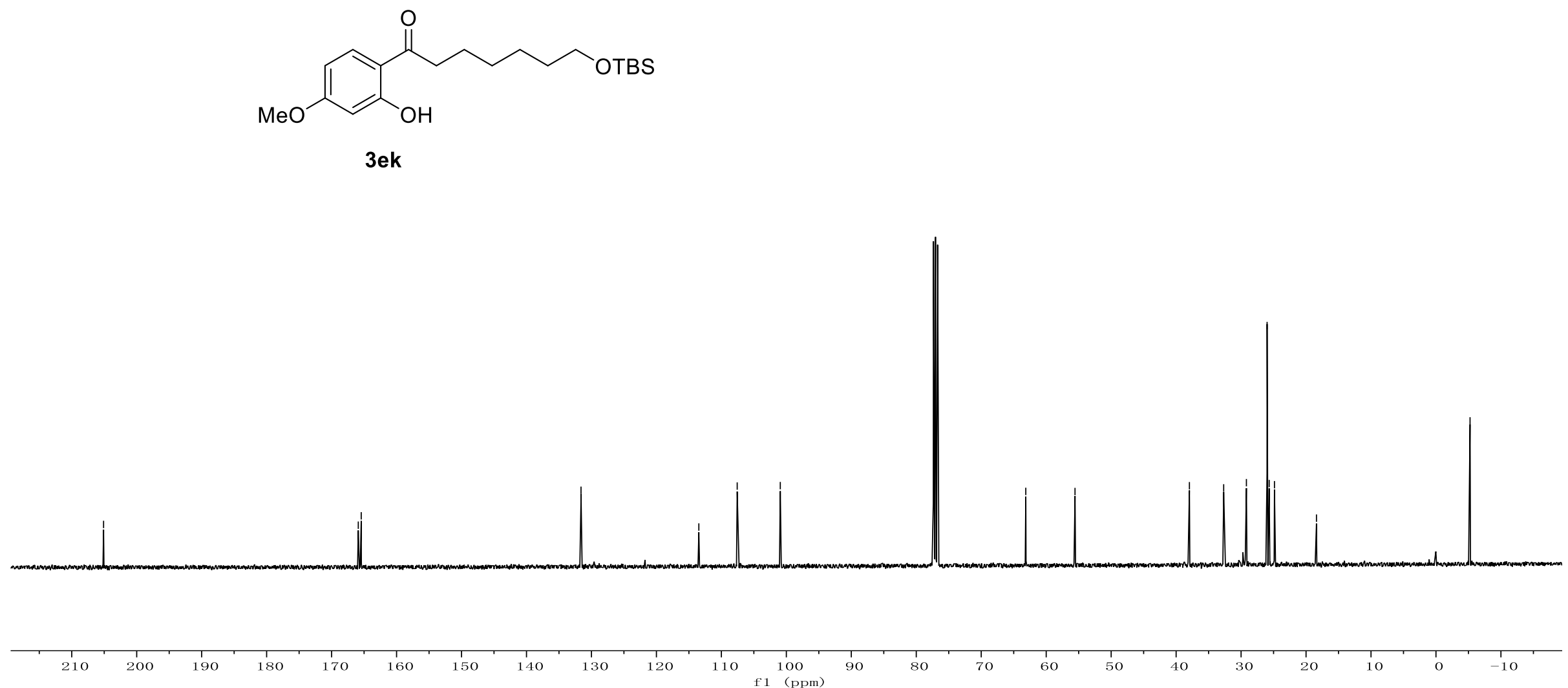
${ }^{1} \mathrm{H}$ NMR-spectrum (400 MHz, $\mathrm{CDCl}_{3}$ ) of $\mathbf{3 f k}$ $\stackrel{\infty}{\ominus}$

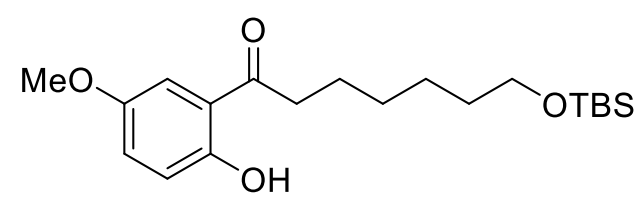

3fk

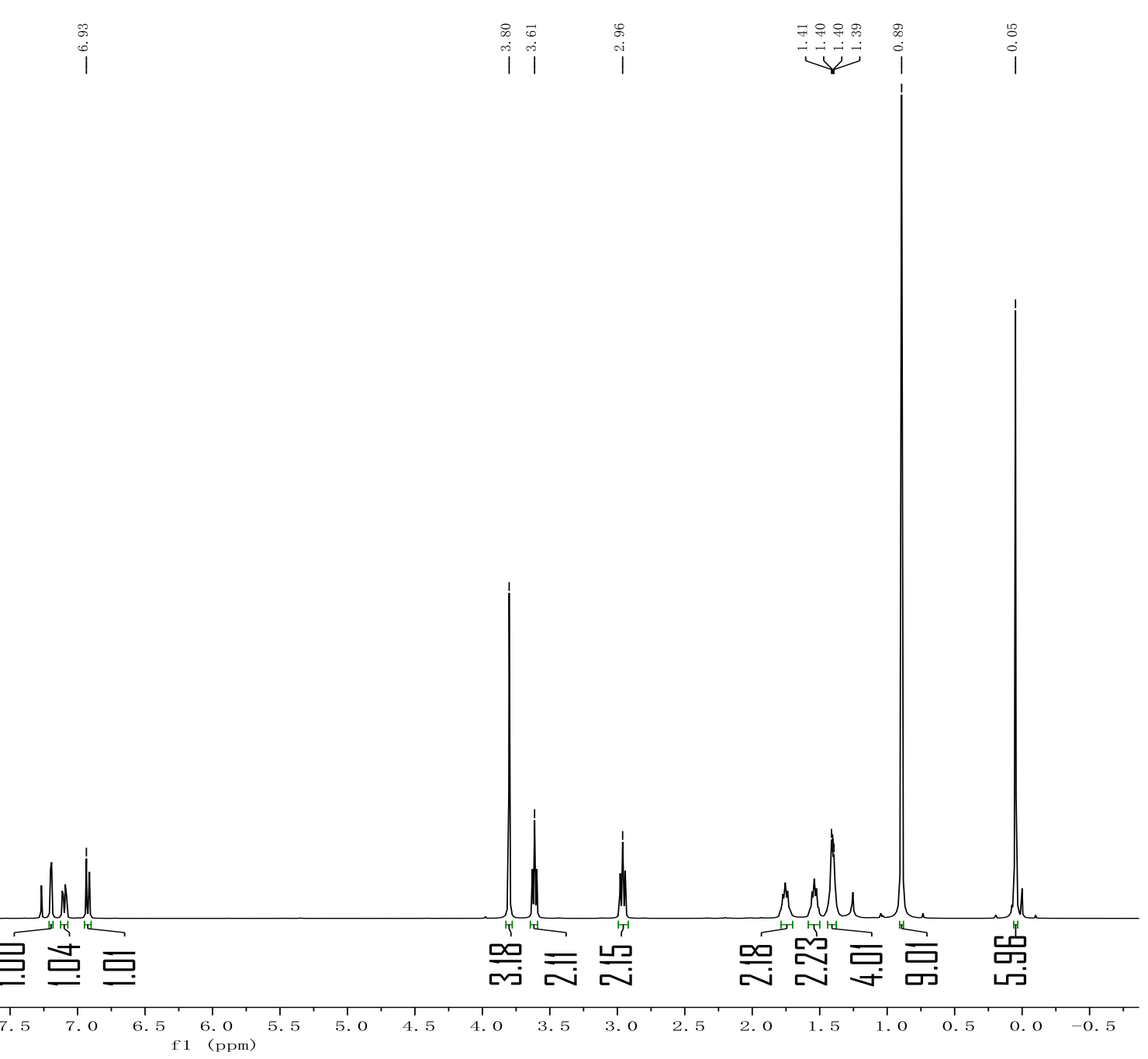


${ }^{13} \mathrm{C}$ NMR-spectrum (101 MHz, $\mathrm{CDCl}_{3}$ ) of $\mathbf{3 f k}$

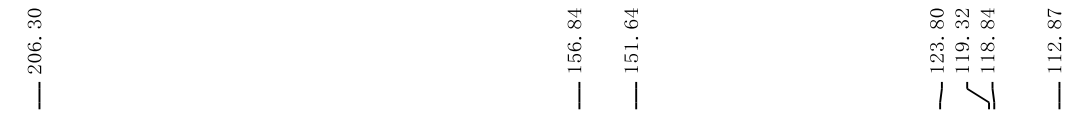

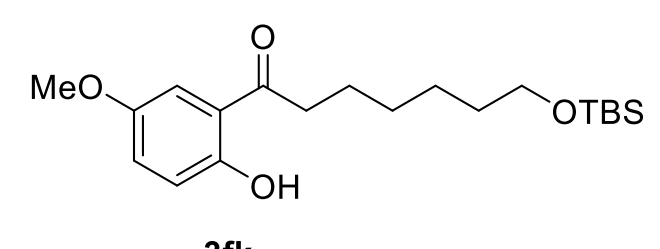

3fk
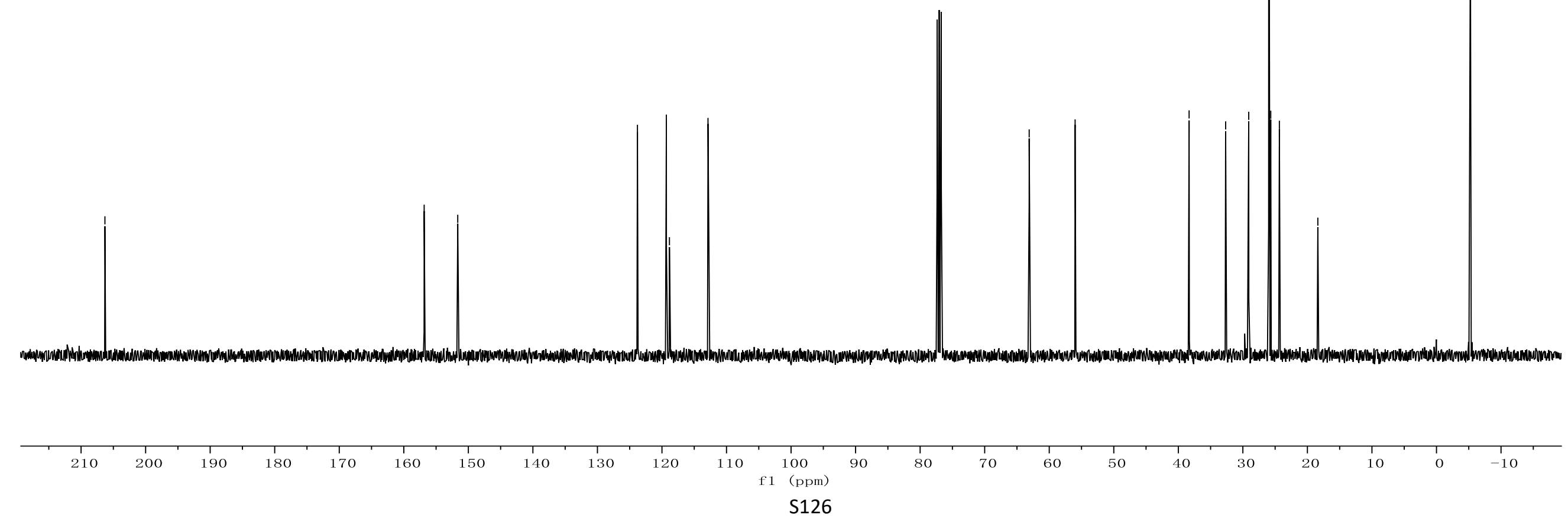
${ }^{1} \mathrm{H}$ NMR-spectrum (400 MHz, $\left.\mathrm{CDCl}_{3}\right)$ of $\mathbf{3 g k}$

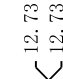

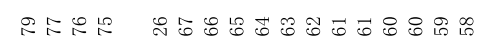

年

3gk

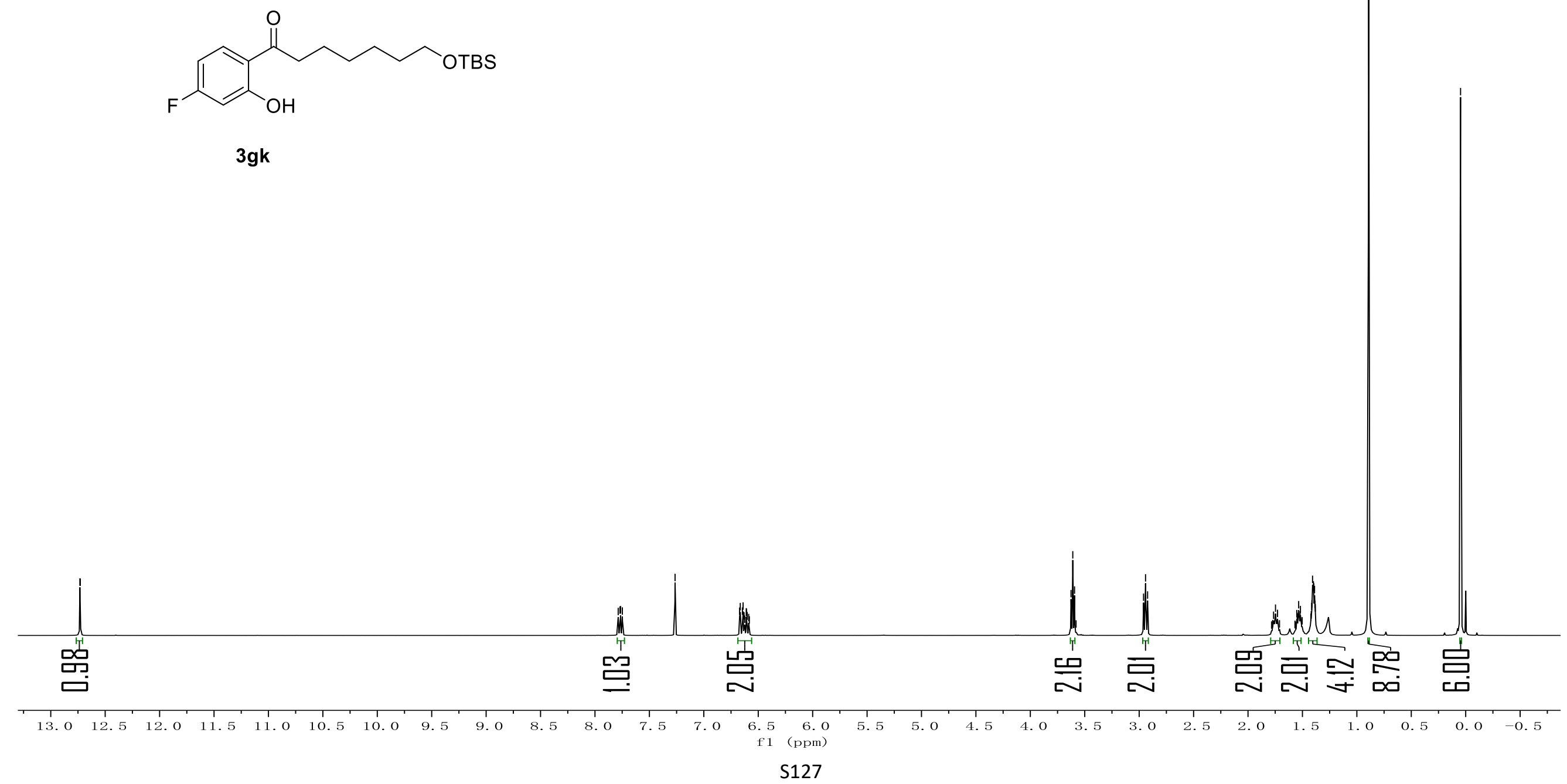

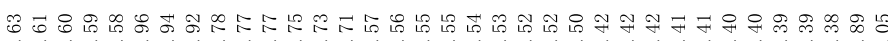
$\underbrace{\infty} n^{\infty} \underbrace{n}$ 
${ }^{13} \mathrm{C}$ NMR-spectrum $\left(101 \mathrm{MHz}, \mathrm{CDCl}_{3}\right.$ ) of $\mathbf{3 g k}$

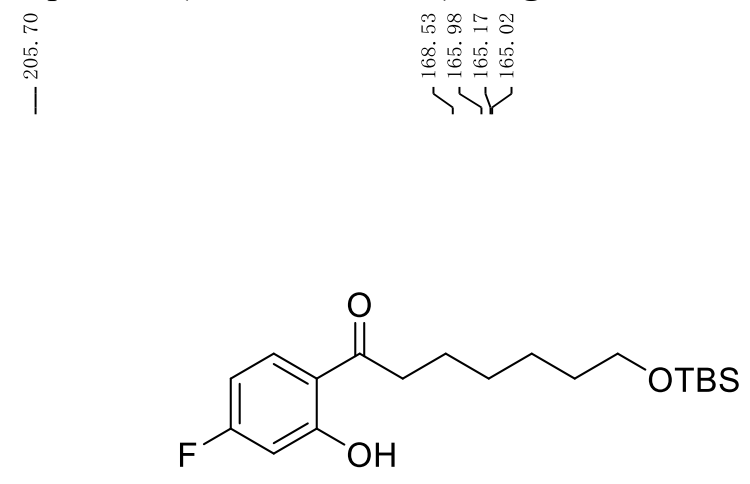

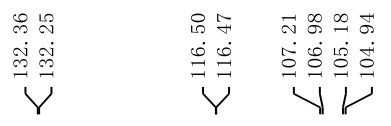

$\underset{\substack{0 \\ 0 \\ 0 \\ 0 \\ 0}}{0}$

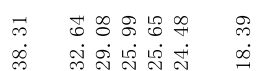

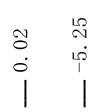

3gk

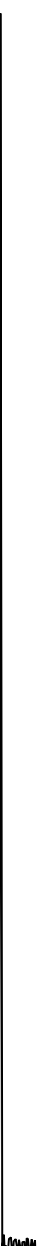

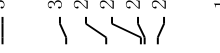
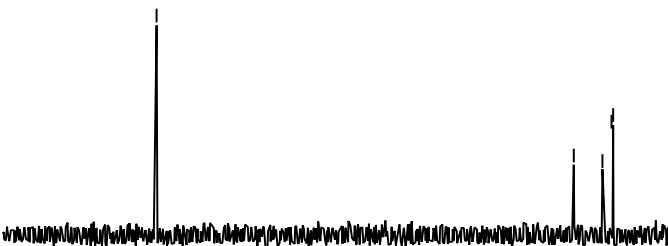

$210 \quad 200$

190

180

$170 \quad 160$

140

130

120

$10 \quad 100$

90

80

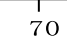


${ }^{19} \mathrm{~F}$ NMR-spectrum $\left(376 \mathrm{MHz}, \mathrm{CDCl}_{3}\right)$ of $\mathbf{3 g k}$
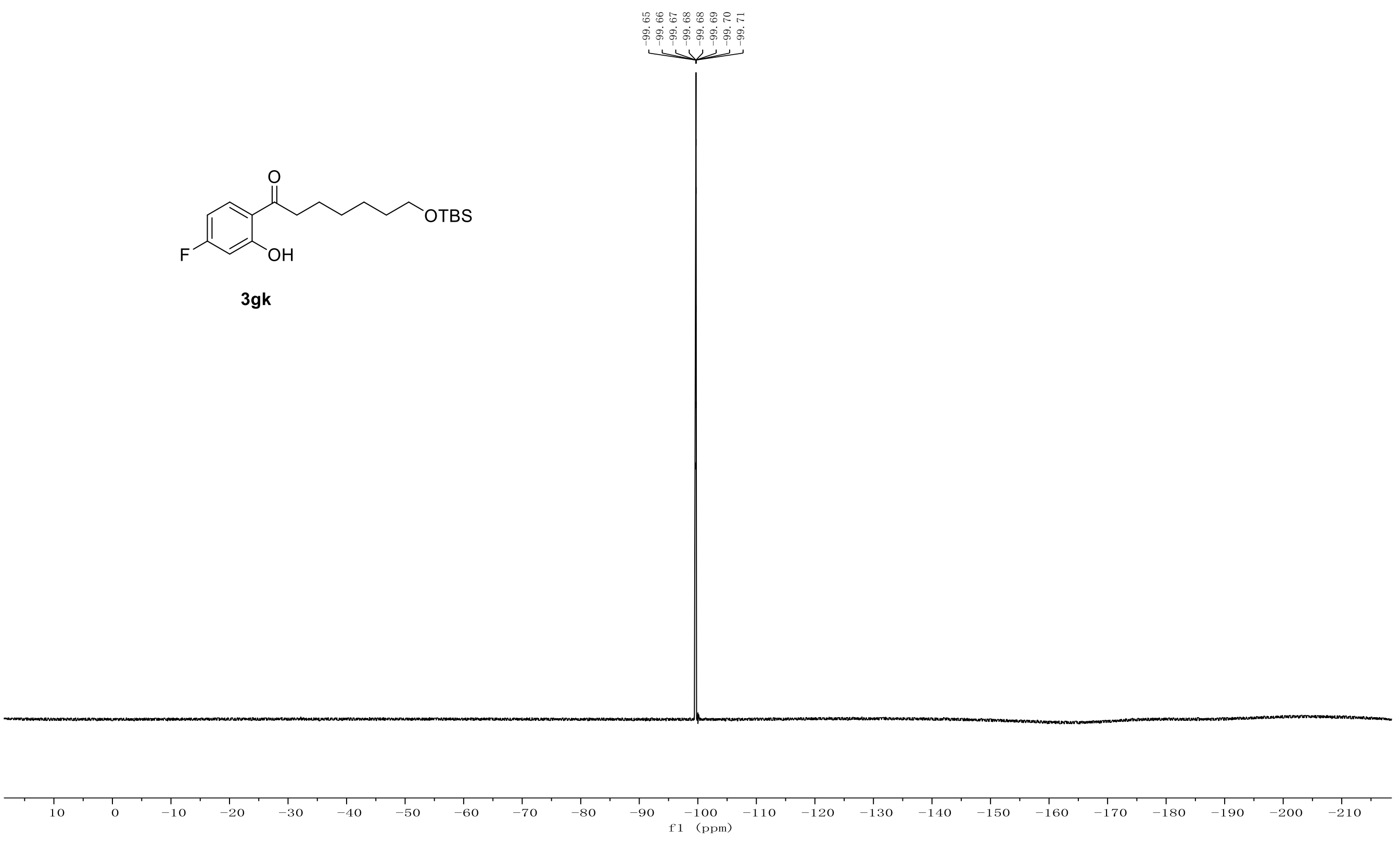
${ }^{1} \mathrm{H}$ NMR-spectrum $\left(400 \mathrm{MHz}, \mathrm{CDCl}_{3}\right)$ of $\mathbf{3 h k}$

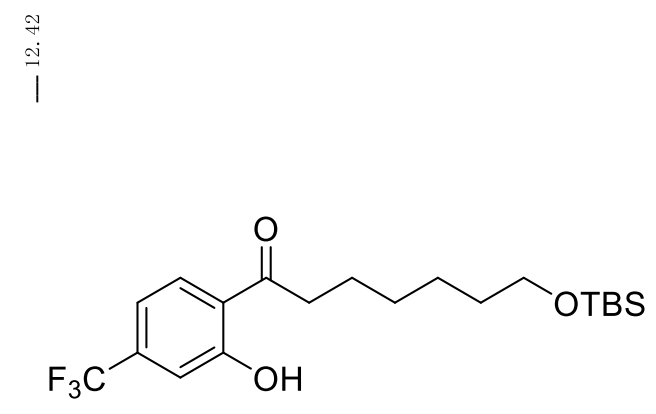

3hk
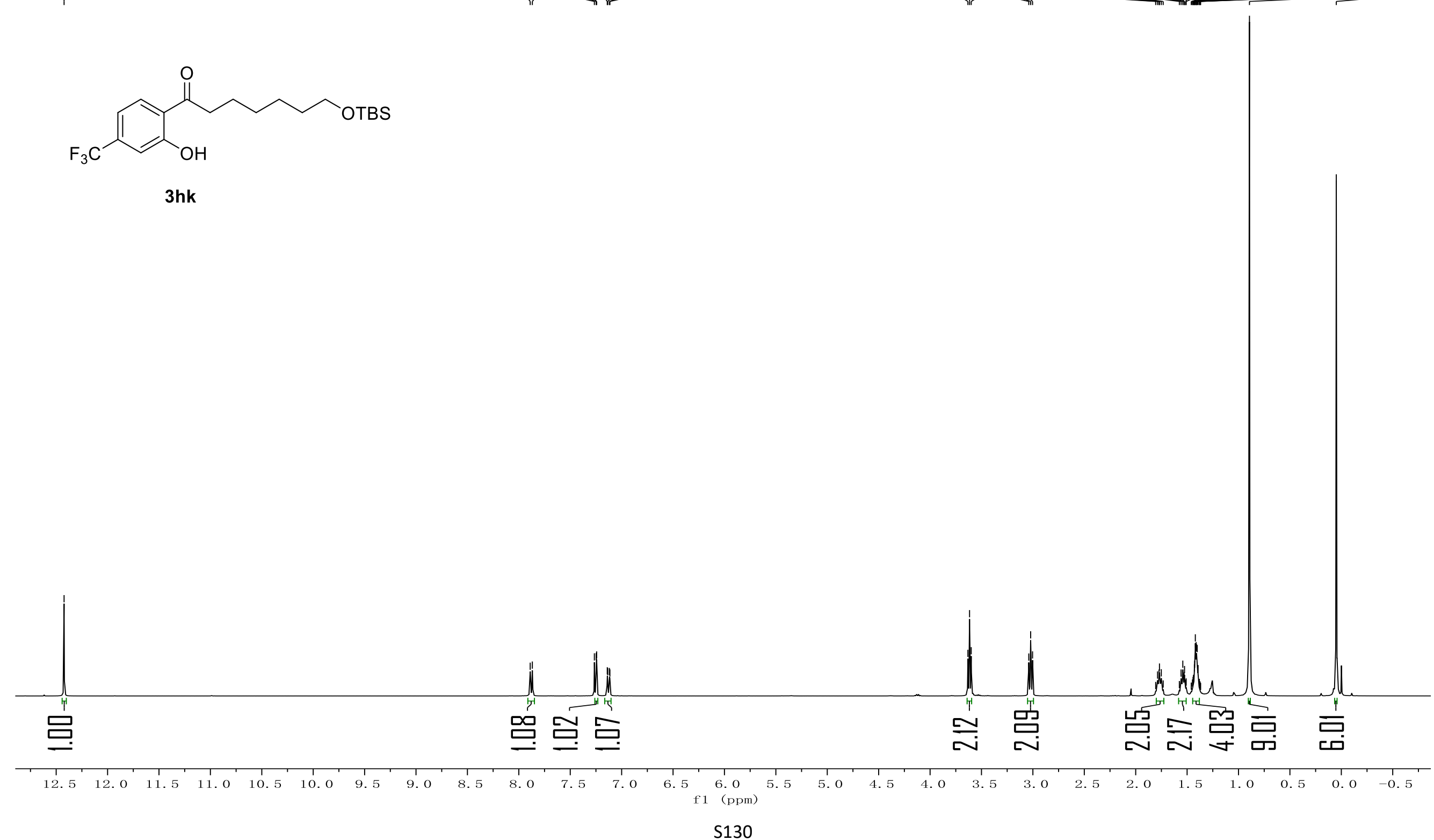
${ }^{13} \mathrm{C} \mathrm{NMR}$-spectrum $\left(101 \mathrm{MHz}, \mathrm{CDCl}_{3}\right)$ of $\mathbf{3 h k}$
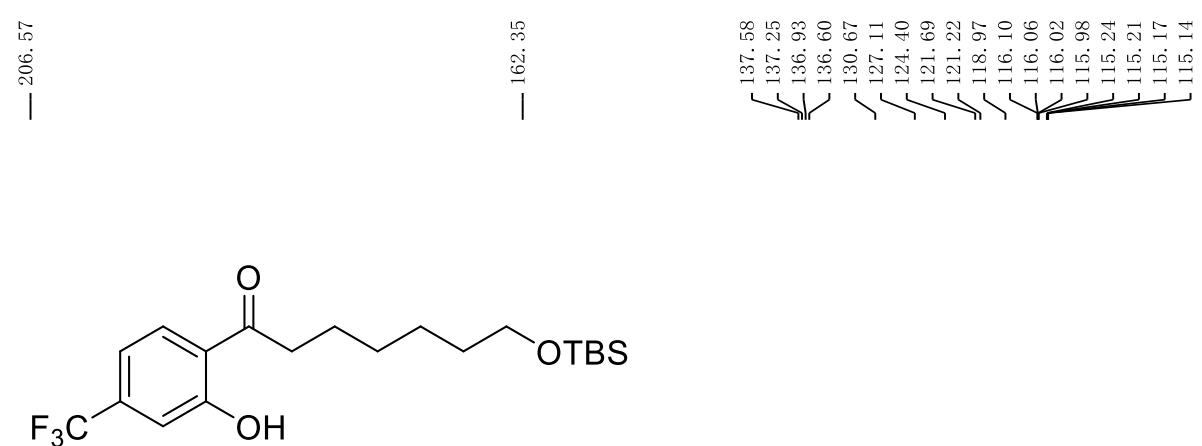

3hk
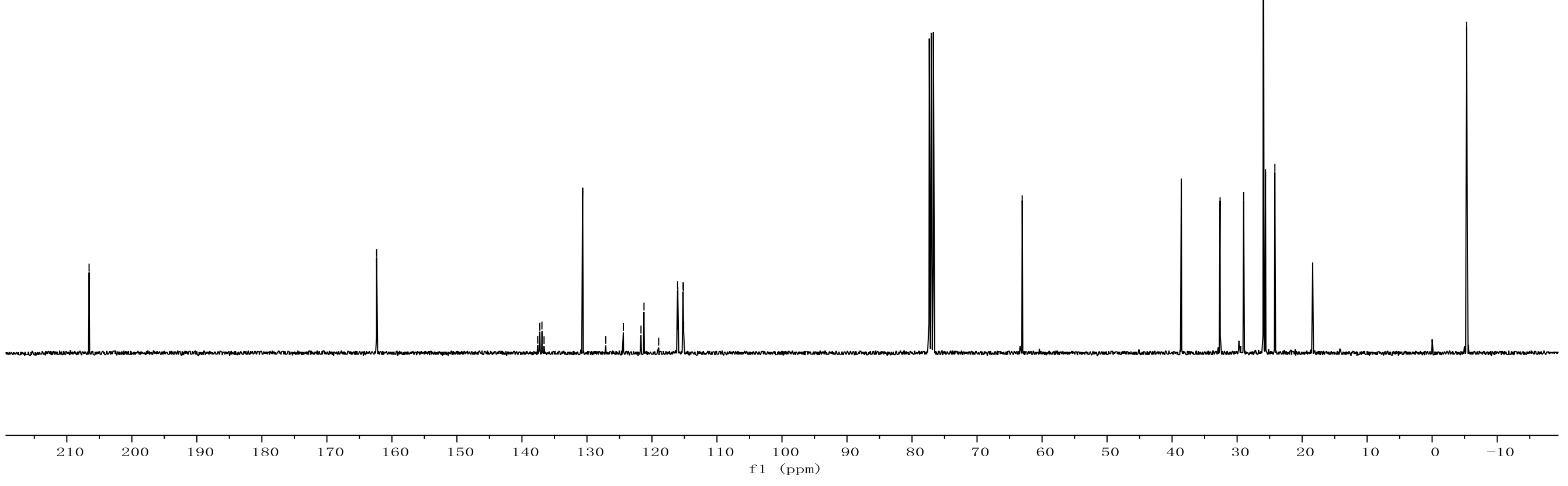
${ }^{19} \mathrm{~F}$ NMR-spectrum $\left(376 \mathrm{MHz}, \mathrm{CDCl}_{3}\right)$ of $\mathbf{3 h k}$

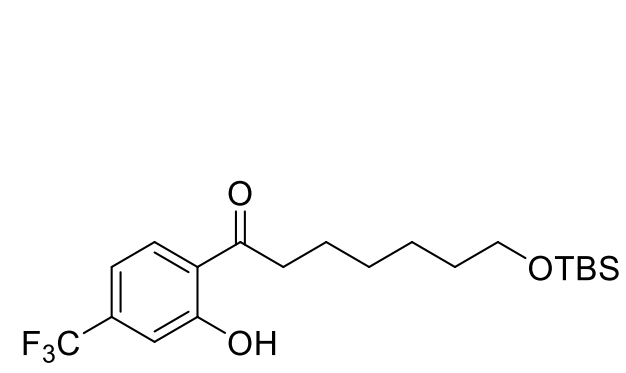

3hk

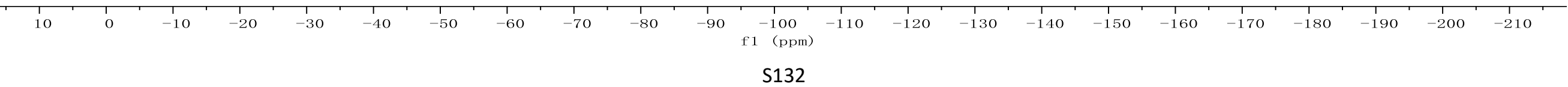


${ }^{1} \mathrm{H}$ NMR-spectrum $\left(500 \mathrm{MHz}, \mathrm{CDCl}_{3}\right)$ of 3ik

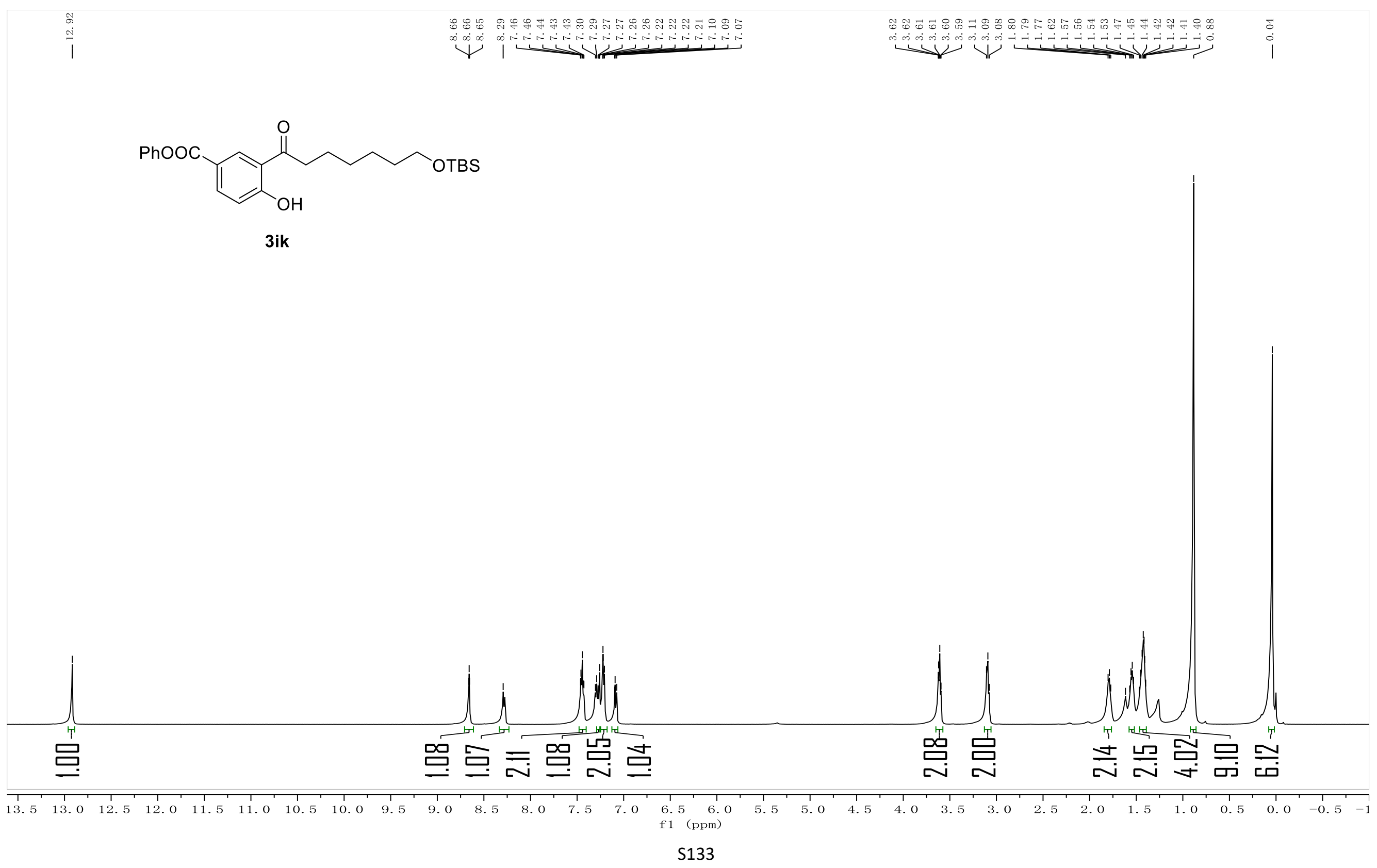


${ }^{13} \mathrm{C}$ NMR-spectrum $\left(101 \mathrm{MHz}, \mathrm{CDCl}_{3}\right)$ of 3ik

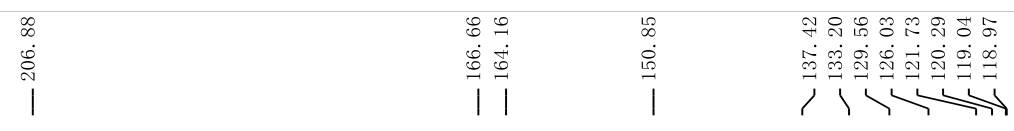<smiles>CC(C)[O+]CCCCCCC(=O)c1cc(C(=O)Oc2ccccc2)ccc1O</smiles>
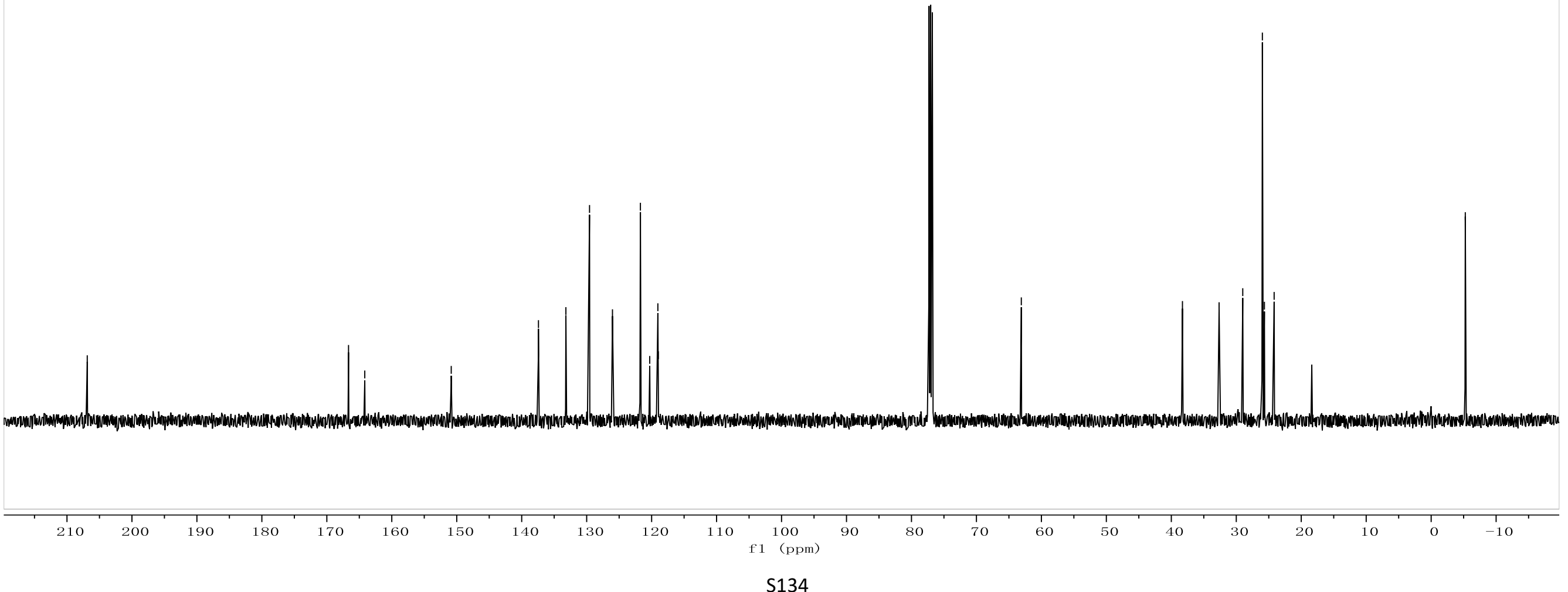

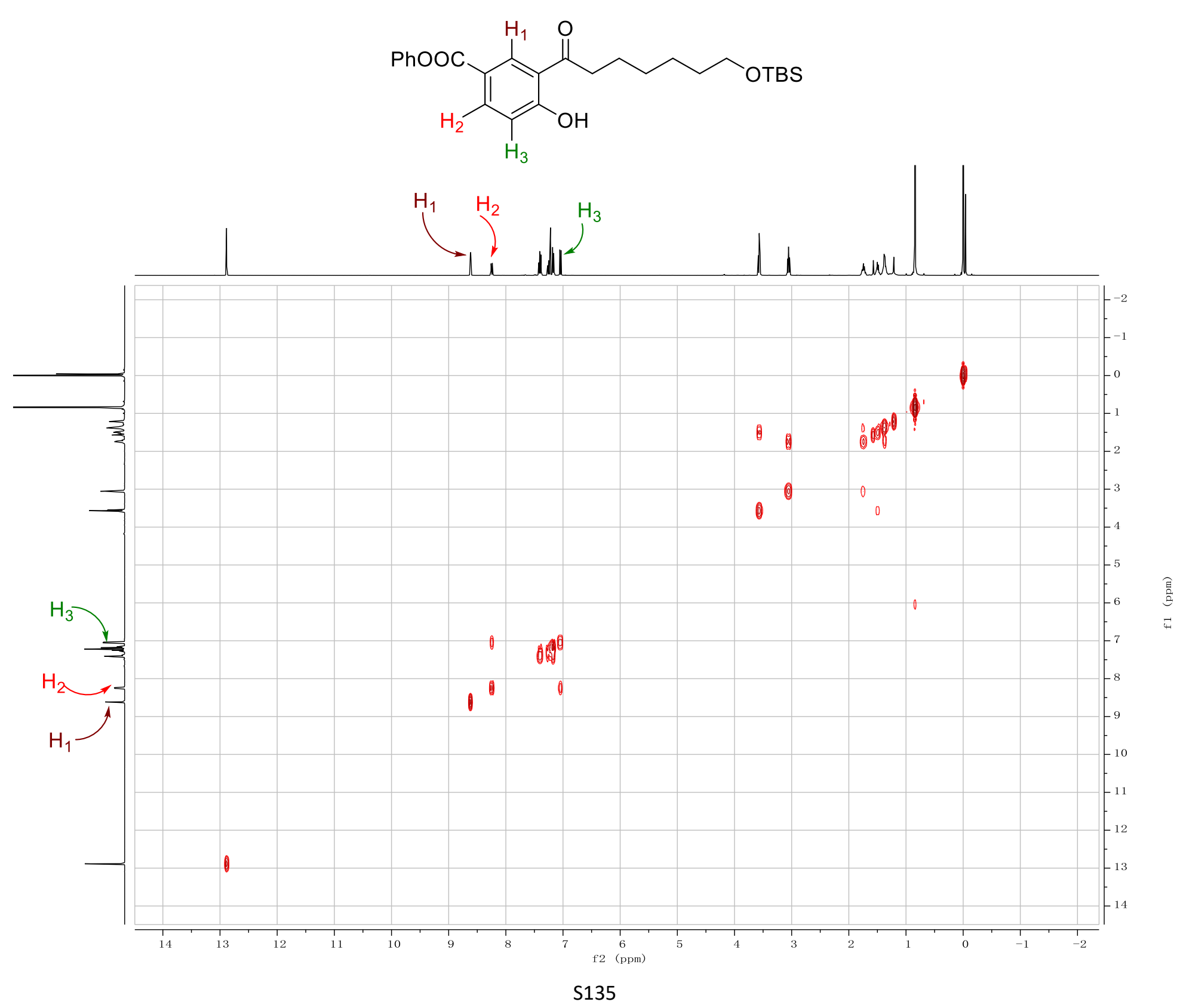


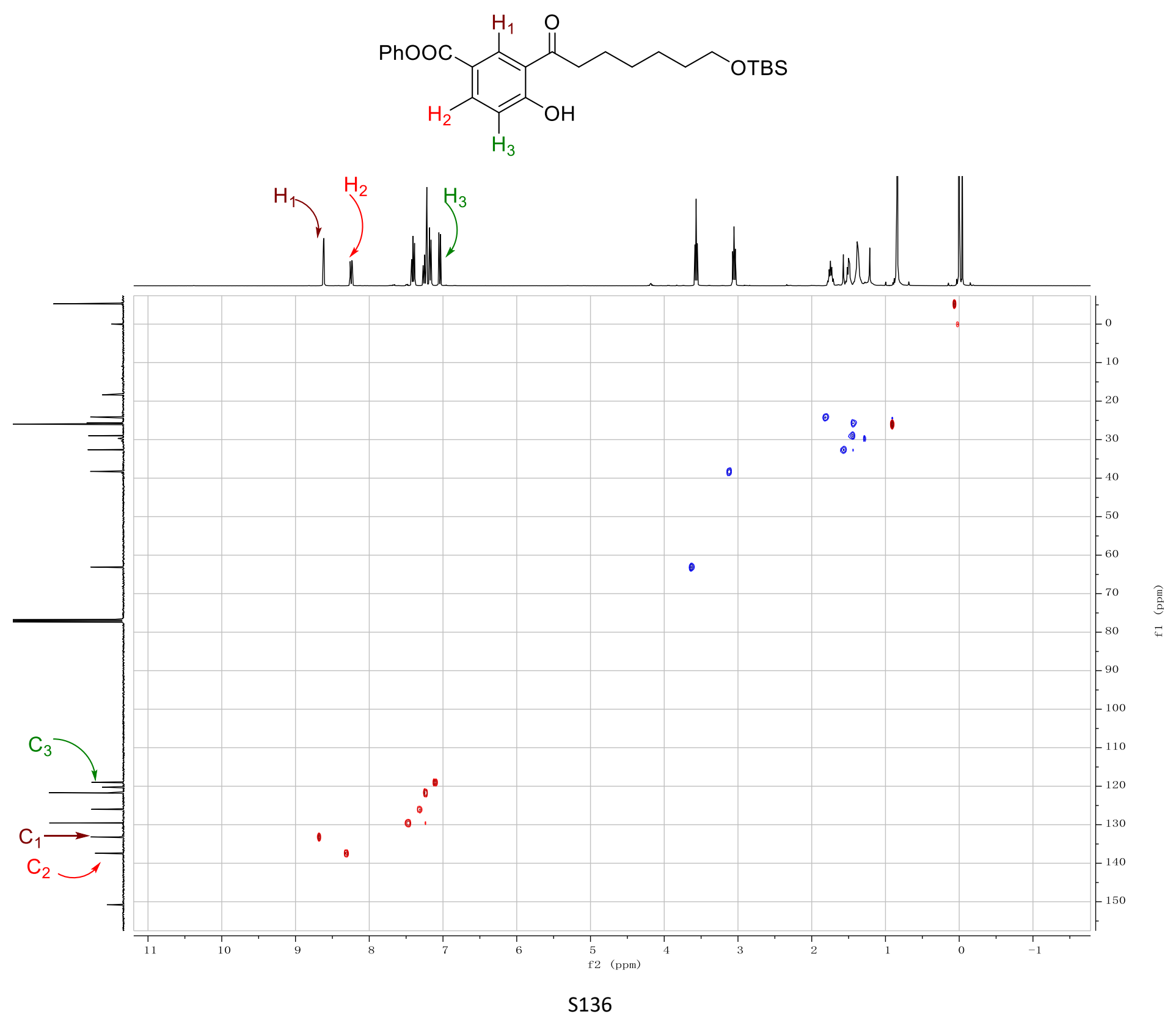




\section{HMBC-spectrum of $\mathbf{3 i k}$}

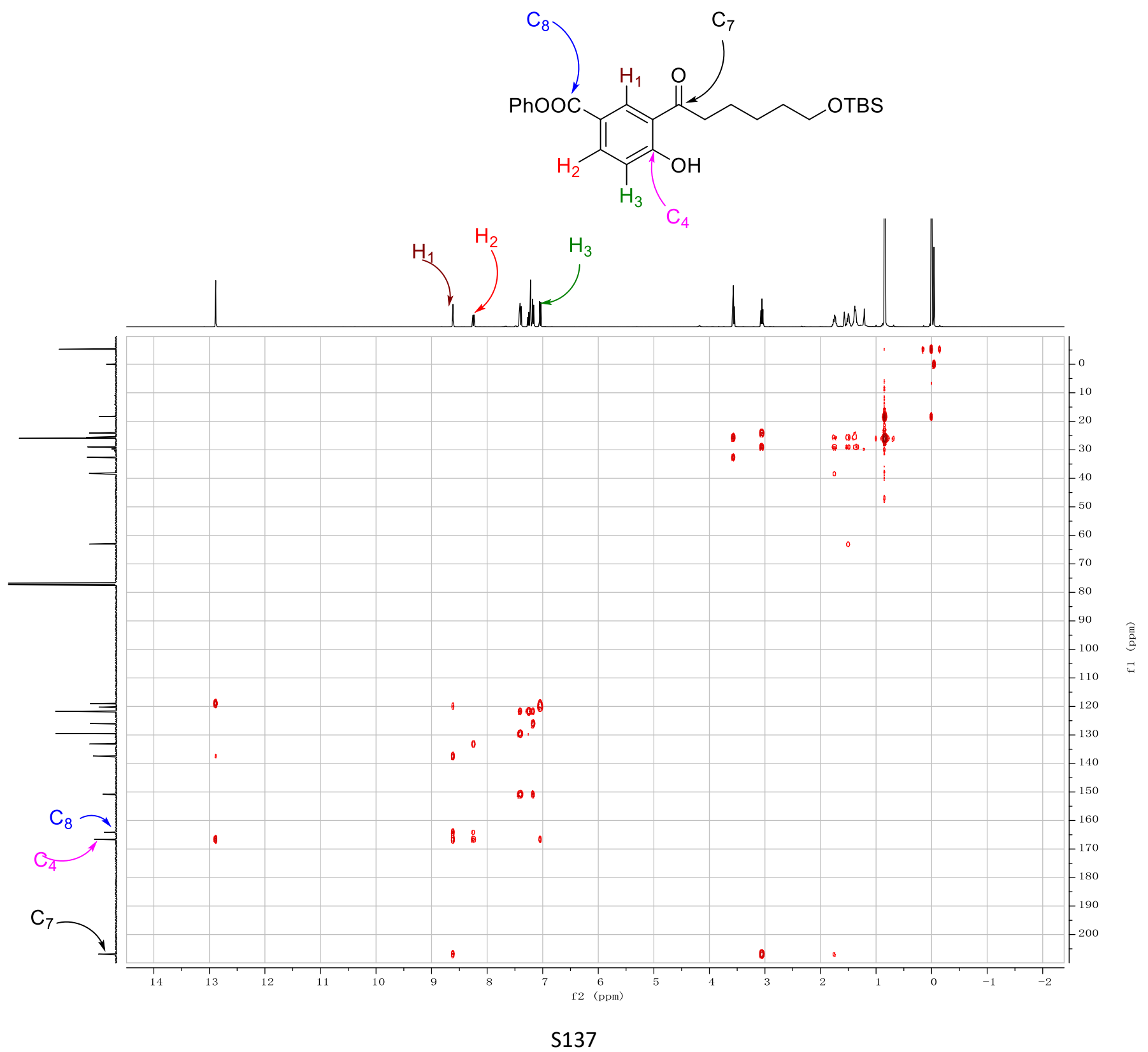


${ }^{1} \mathrm{H}$ NMR-spectrum $\left(400 \mathrm{MHz}, \mathrm{CDCl}_{3}\right)$ of $\mathbf{3 j k}$

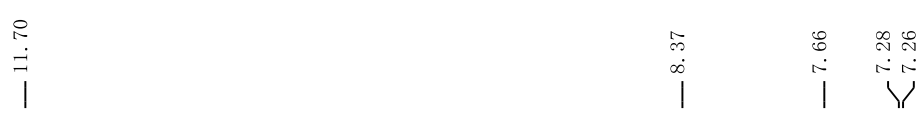

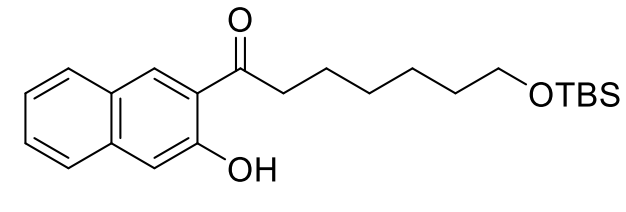

3jk
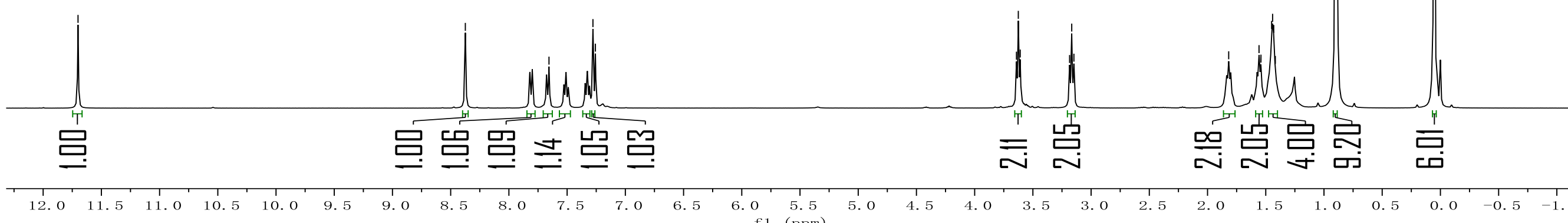
${ }^{13} \mathrm{CNMR}$-spectrum $\left(101 \mathrm{MHz}, \mathrm{CDCl}_{3}\right)$ of $\mathbf{3 j k}$
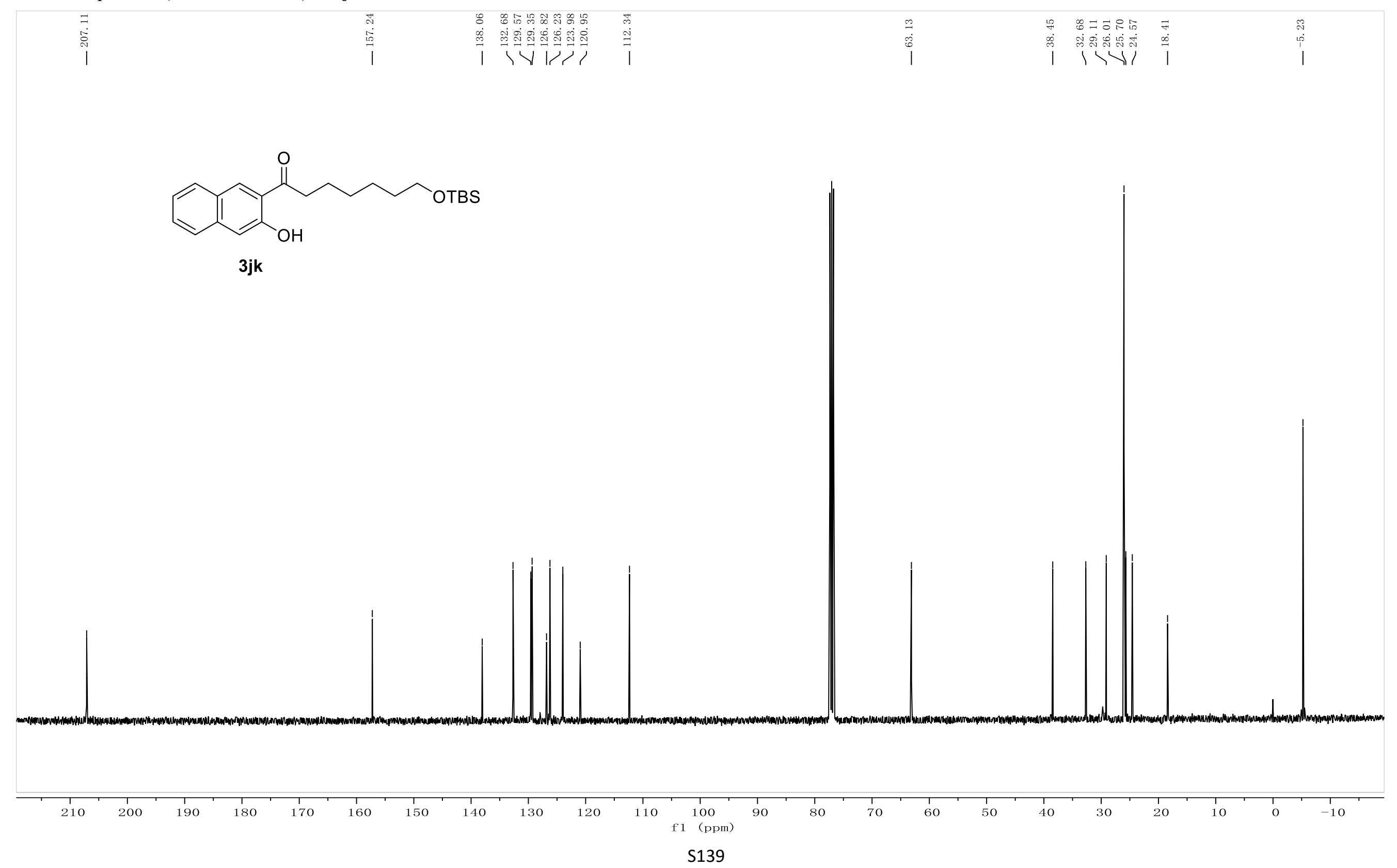
${ }^{1} \mathrm{H}$ NMR-spectrum $\left(500 \mathrm{MHz}, \mathrm{CDCl}_{3}\right)$ of $\mathbf{3 k b}$

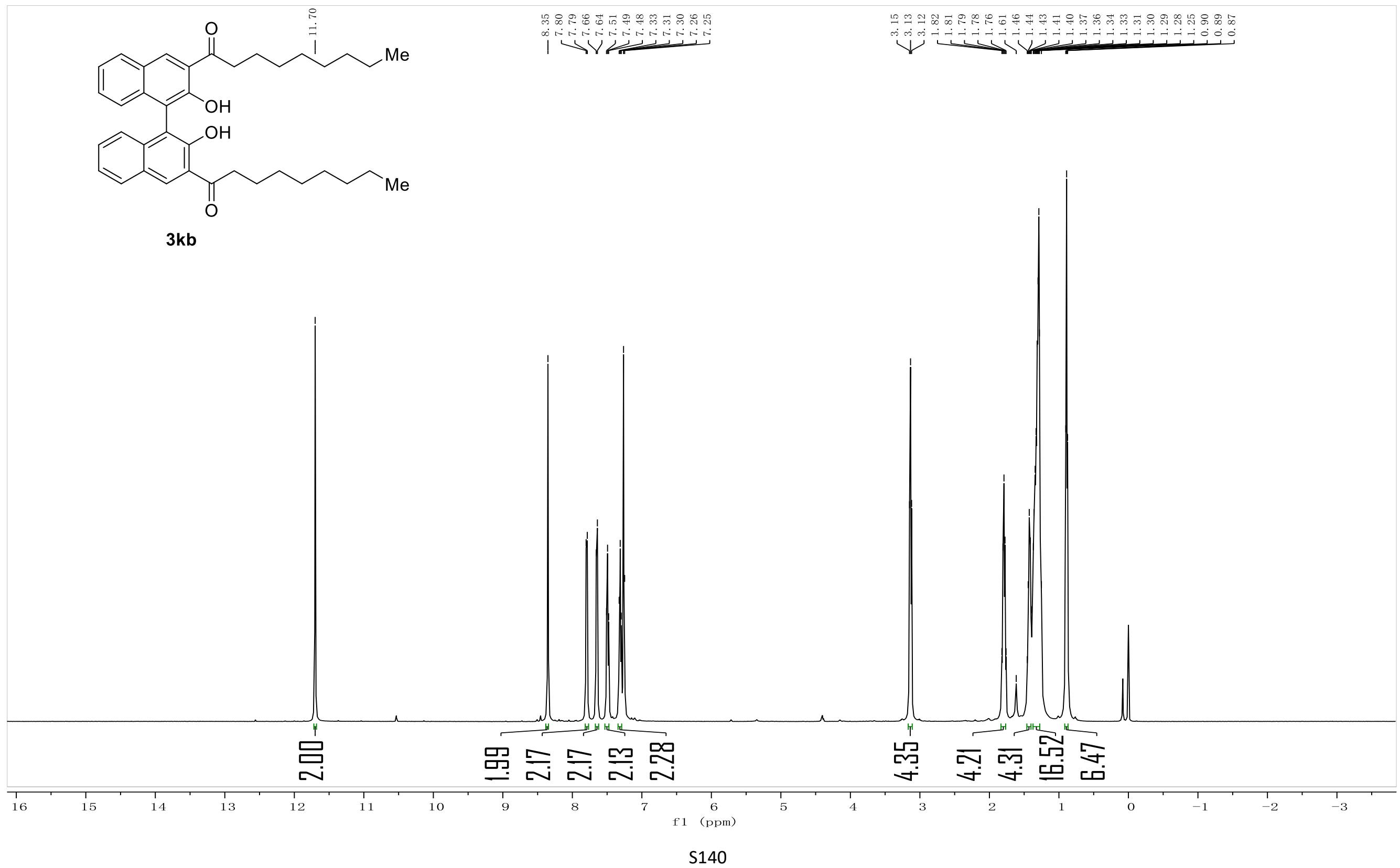


${ }^{13} \mathrm{CNMR}$-spectrum $\left(126 \mathrm{MHz}, \mathrm{CDCl}_{3}\right)$ of $\mathbf{3 k b}$
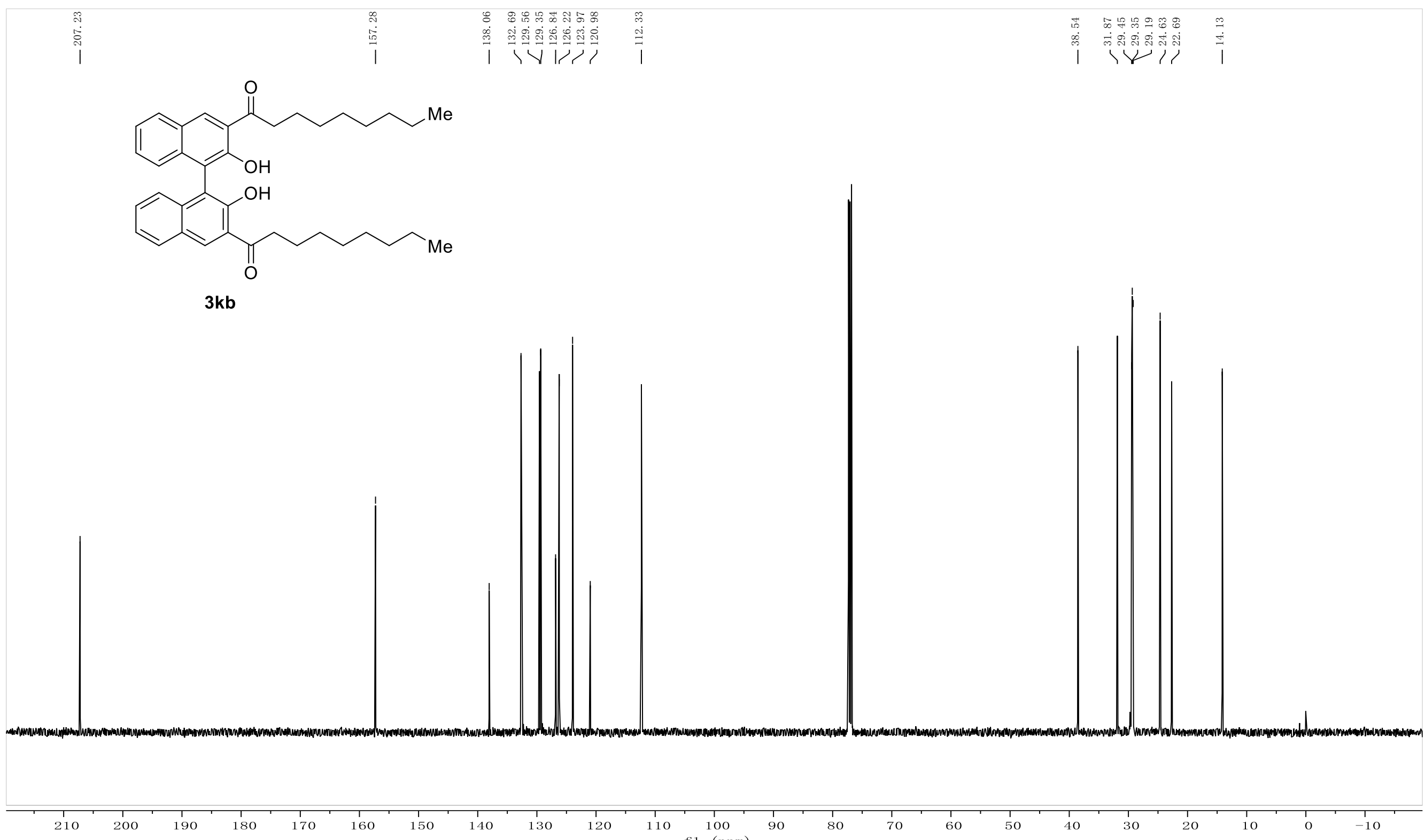

120

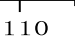

100

1
$90 \quad 80$
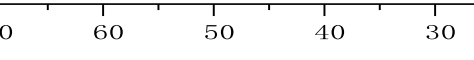

20

o 
${ }^{1} \mathrm{H}$ NMR-spectrum $\left(500 \mathrm{MHz}, \mathrm{CDCl}_{3}\right)$ of $\mathbf{3 i b}$

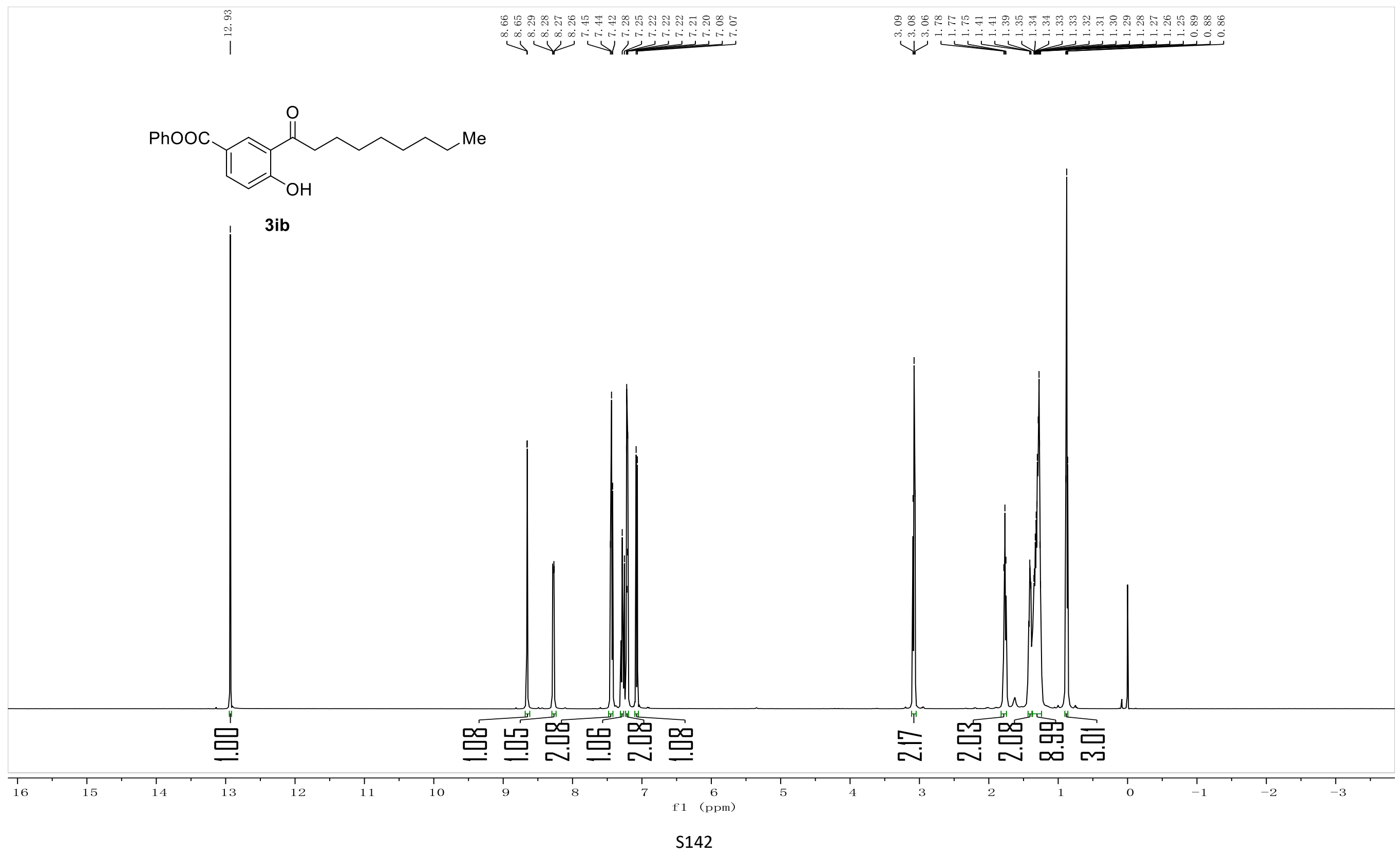


${ }^{13} \mathrm{CNMR}$-spectrum $\left(126 \mathrm{MHz}, \mathrm{CDCl}_{3}\right)$ of $\mathbf{3 i b}$

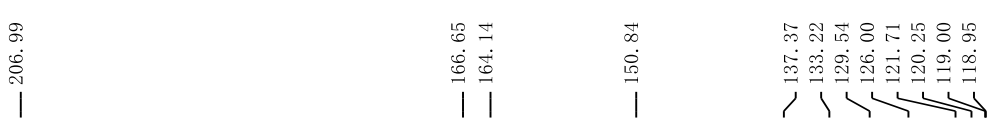

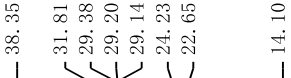

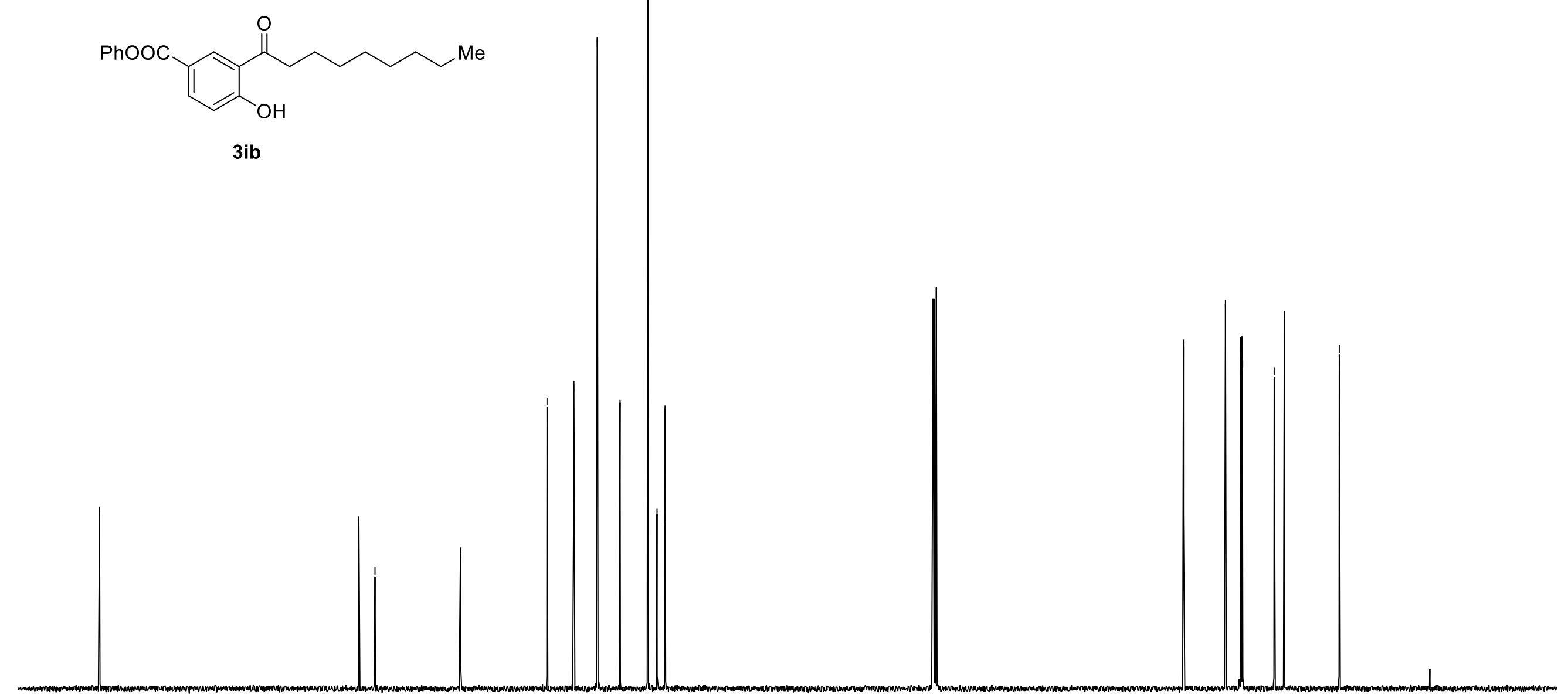

3ib
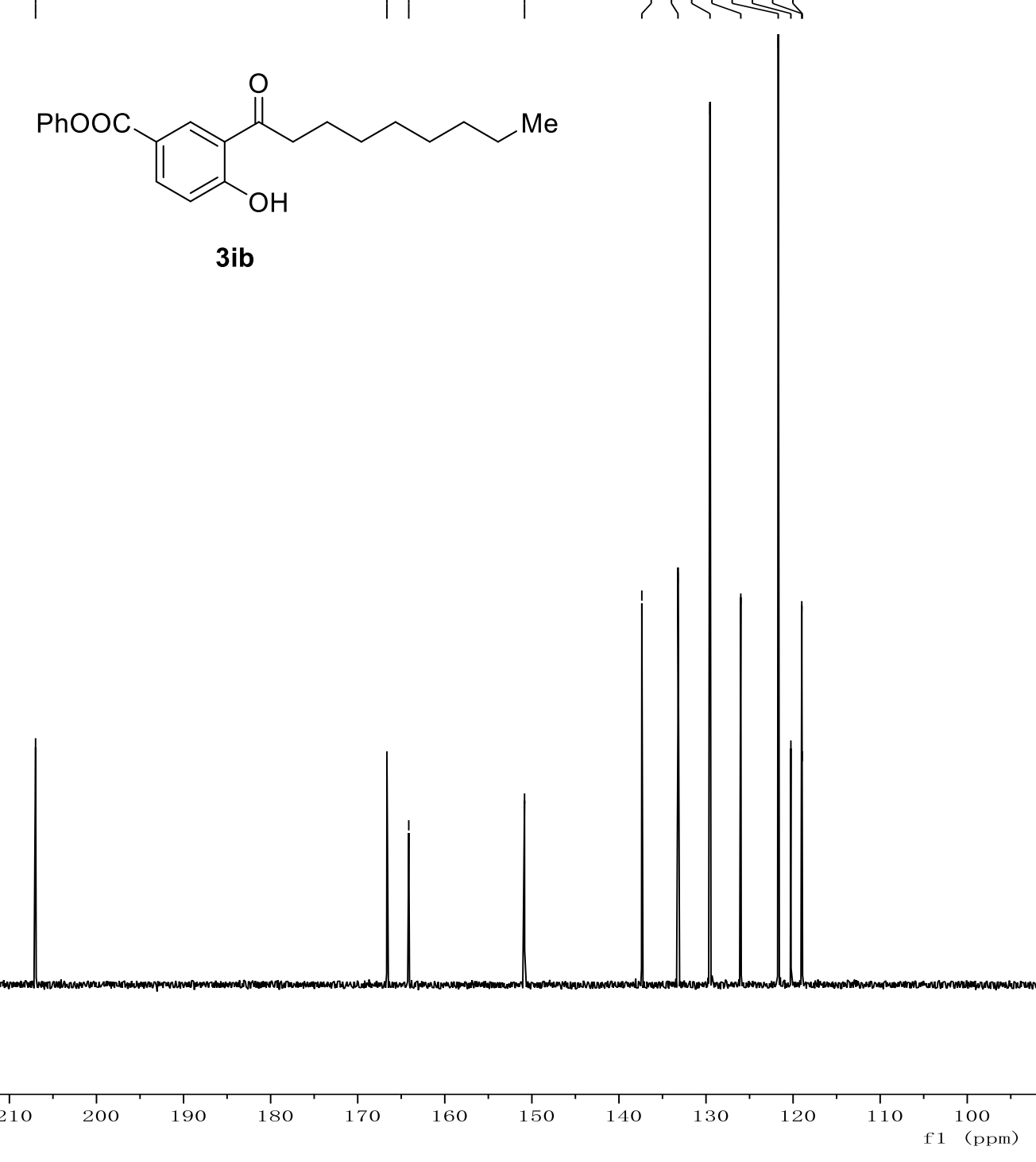
COSY-spectrum of $\mathbf{3 i b}$
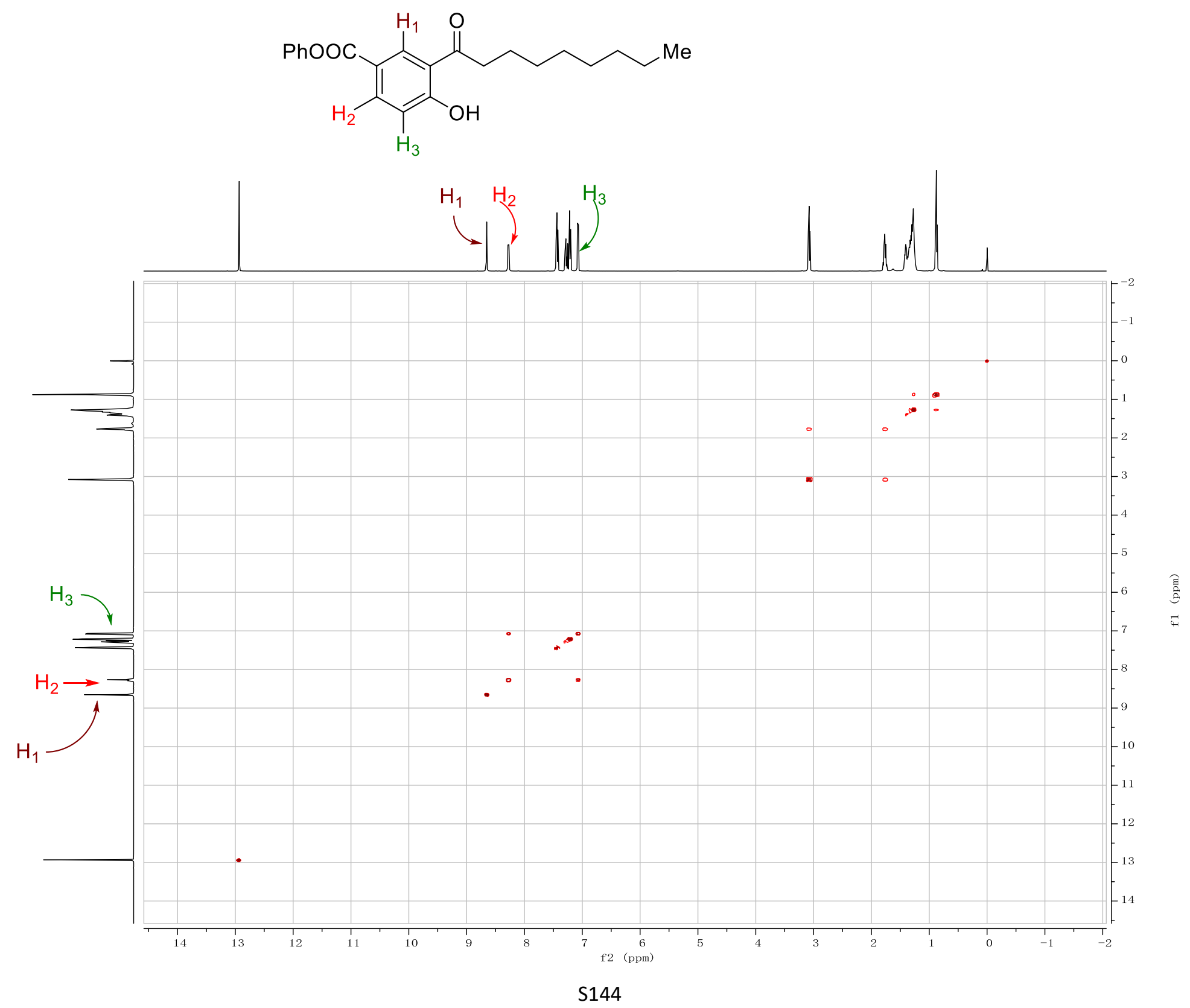
HSQC-spectrum of 3ib
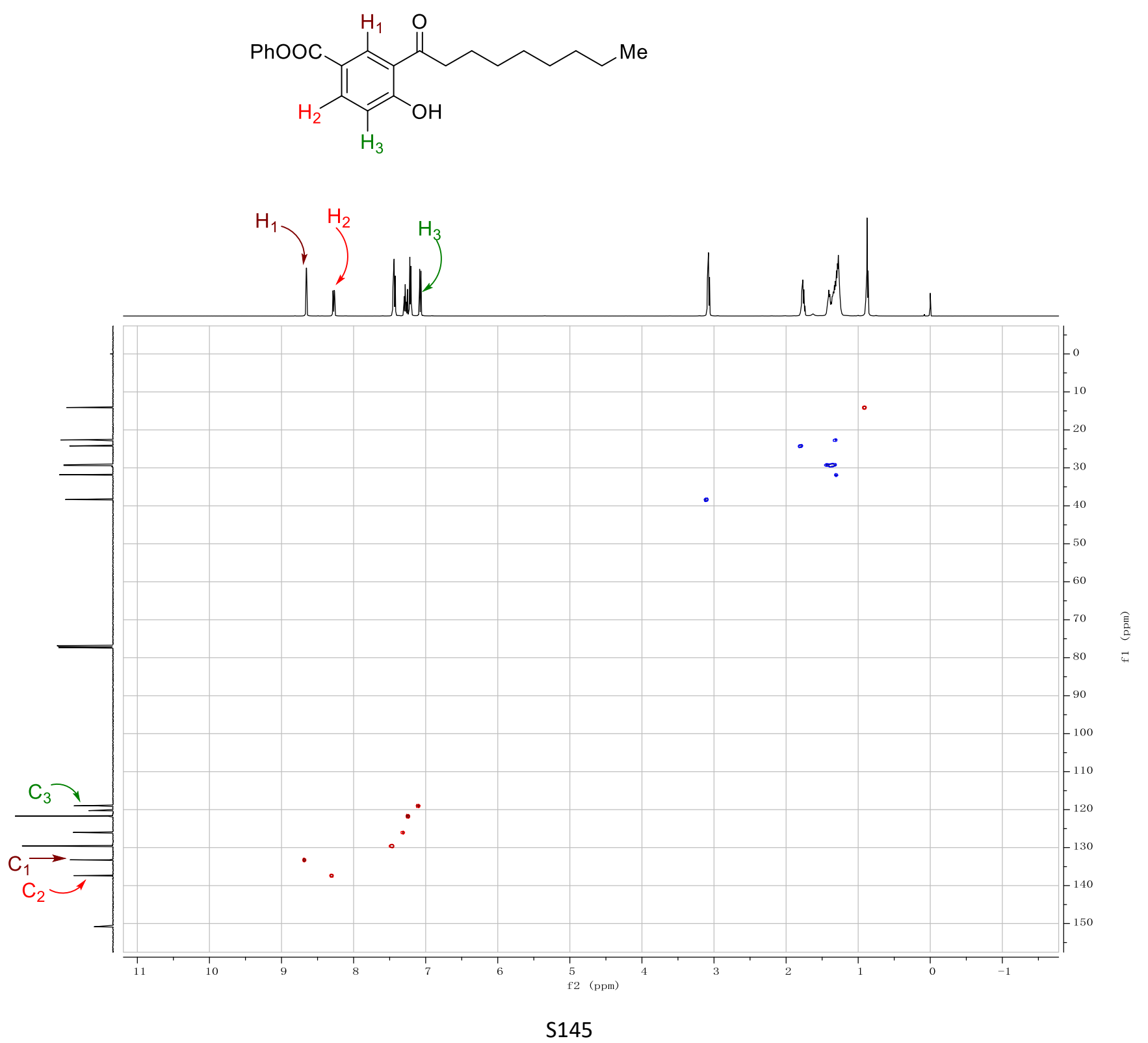


\section{HMBC-spectrum of $\mathbf{3 i b}$}
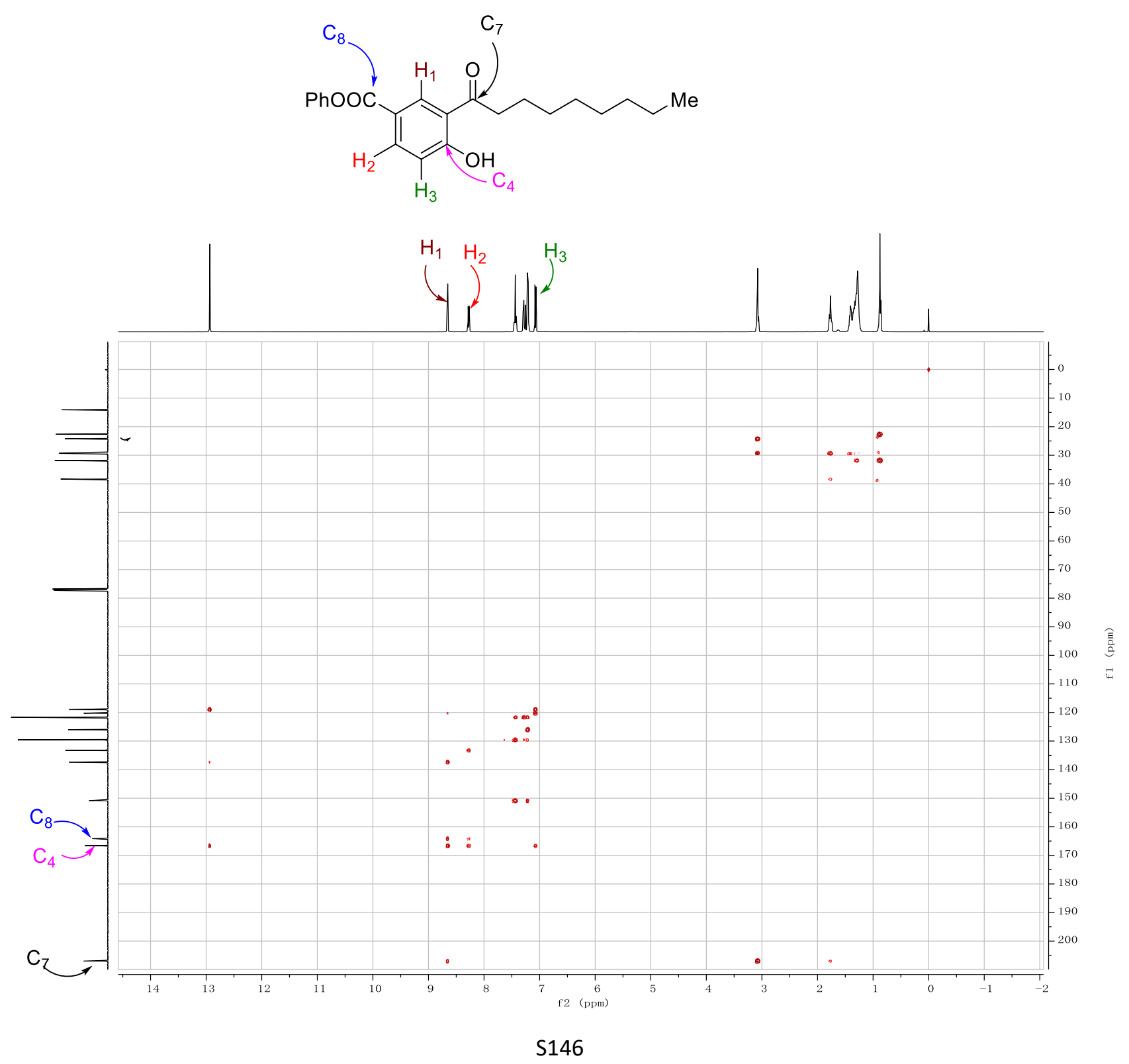
${ }^{1} \mathrm{H}$ NMR-spectrum $\left(400 \mathrm{MHz}, \mathrm{CDCl}_{3}\right)$ of $\mathbf{5 a b}$

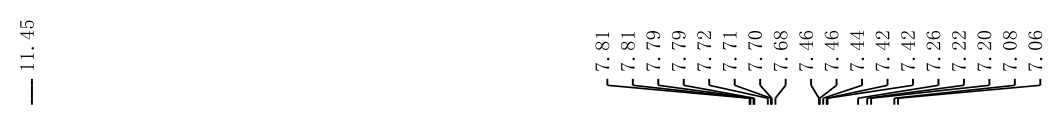

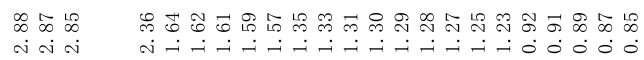

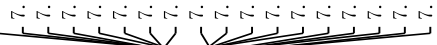

1

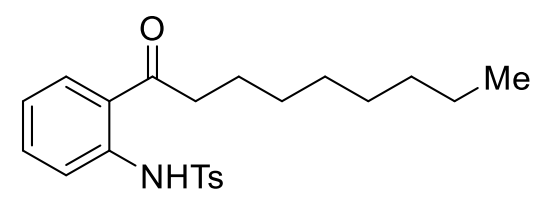

$5 a b$
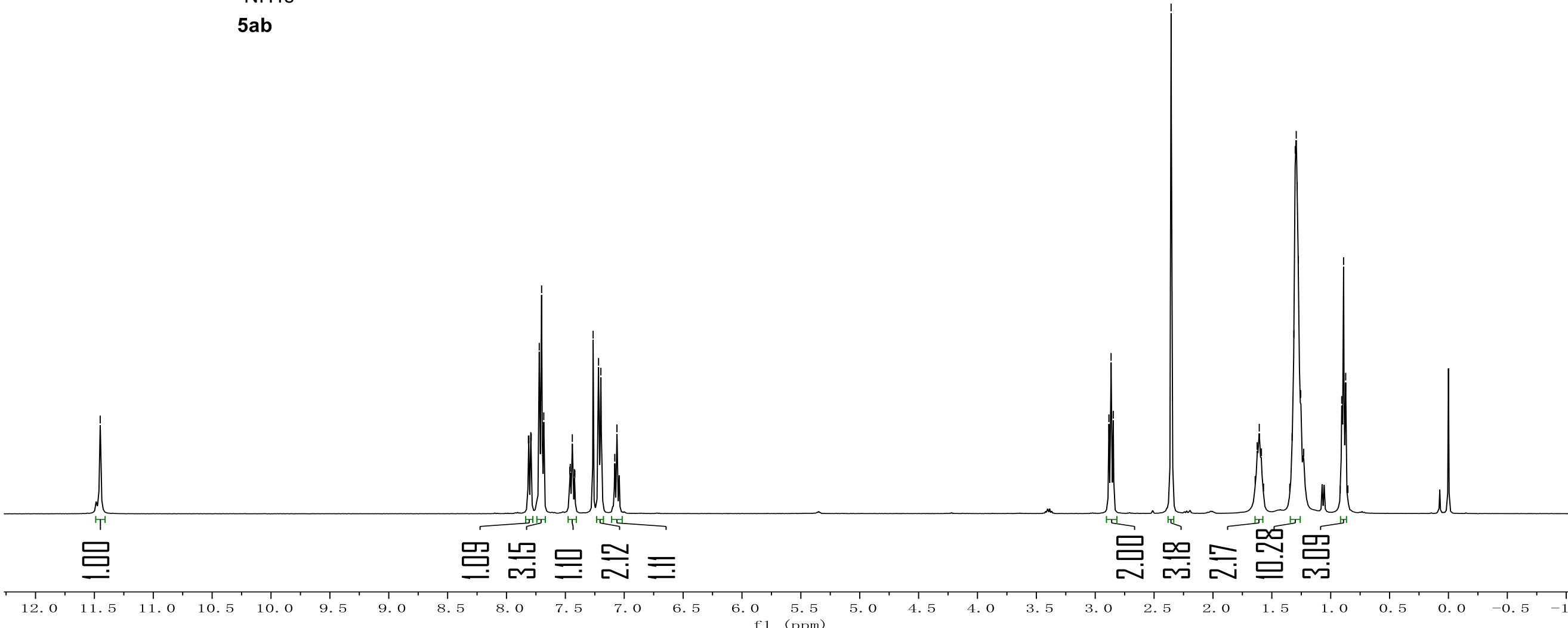
${ }^{13} \mathrm{CNMR}$-spectrum $\left(101 \mathrm{MHz}, \mathrm{CDCl}_{3}\right)$ of $\mathbf{5 a b}$

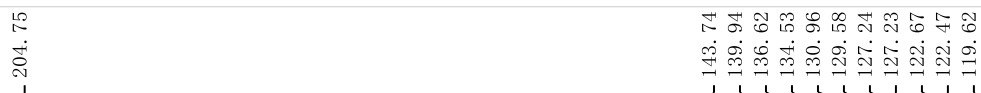

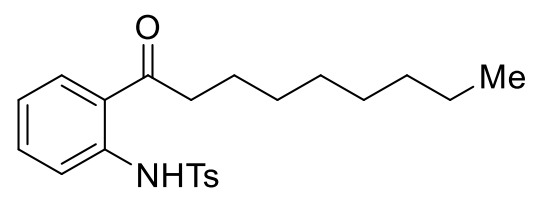

$5 a b$

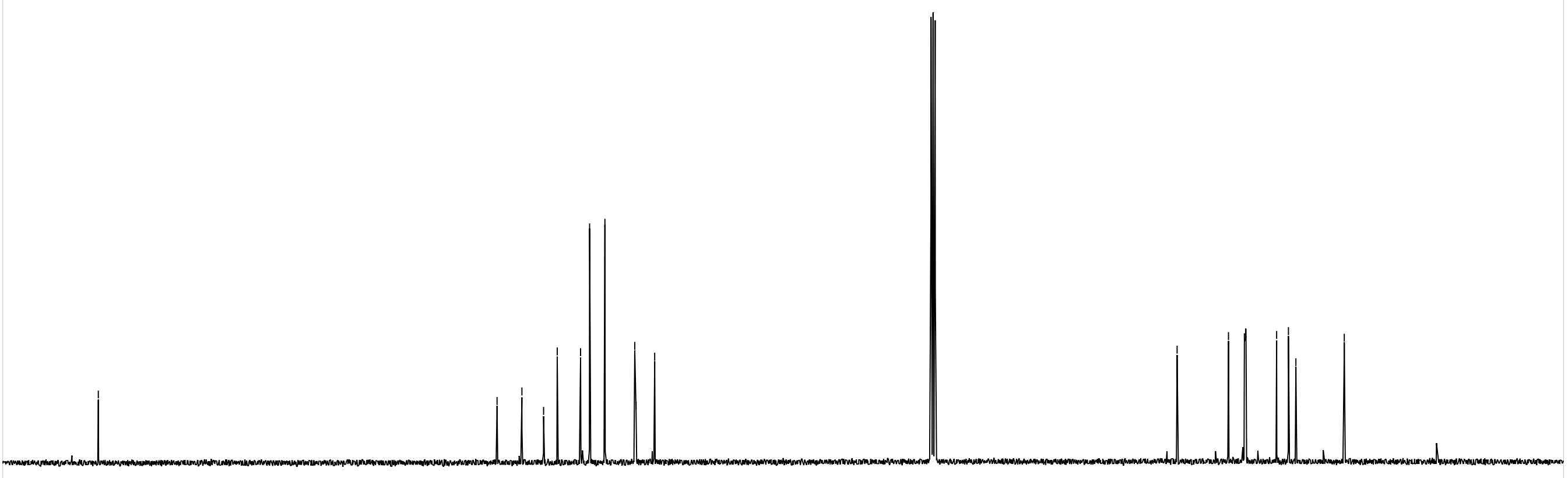


${ }^{1} \mathrm{H}$ NMR-spectrum $\left(400 \mathrm{MHz}, \mathrm{CDCl}_{3}\right)$ of $\mathbf{5 a c}$

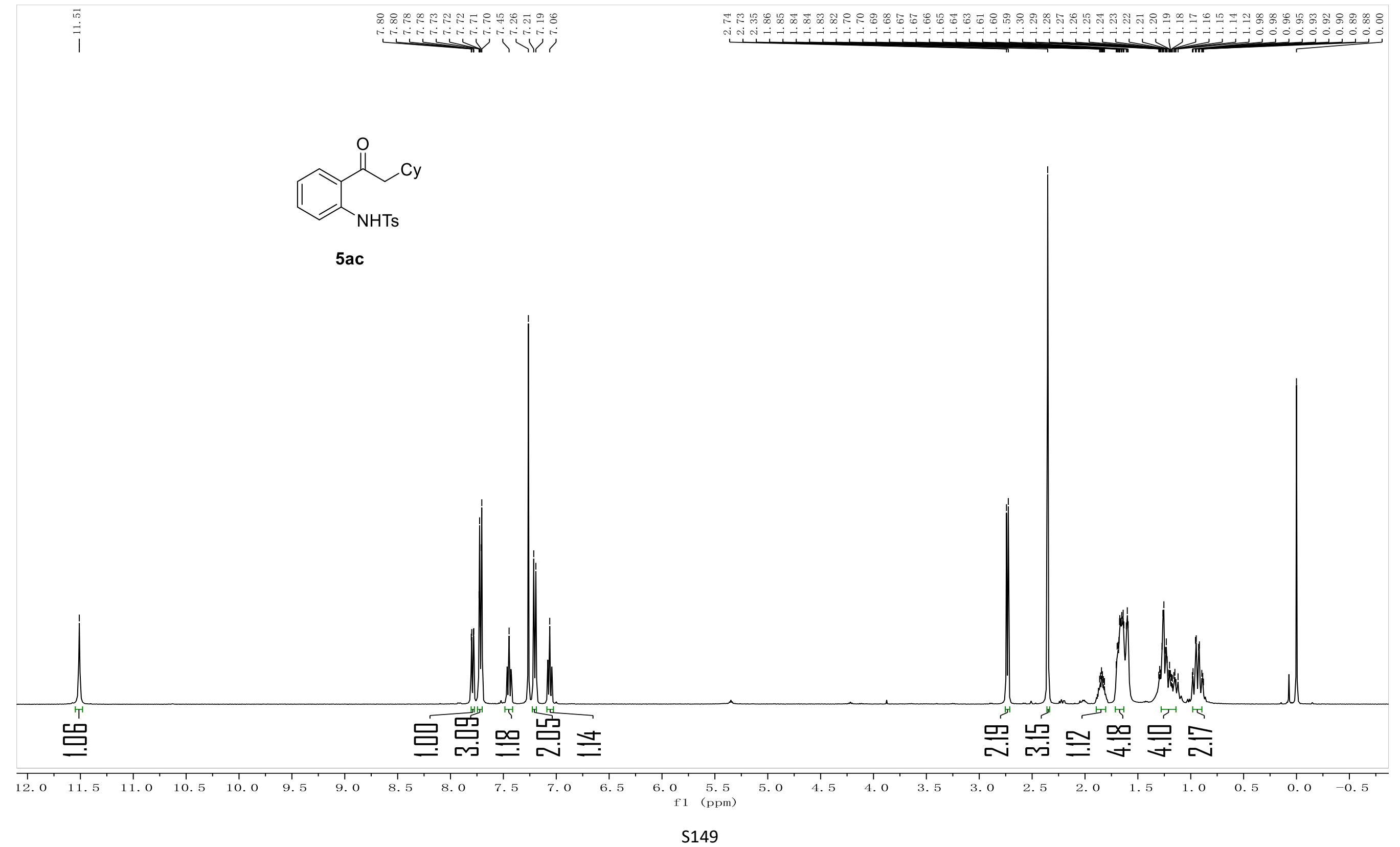


${ }^{13} \mathrm{CNMR}$-spectrum $\left(101 \mathrm{MHz}, \mathrm{CDCl}_{3}\right)$ of $\mathbf{5 a c}$
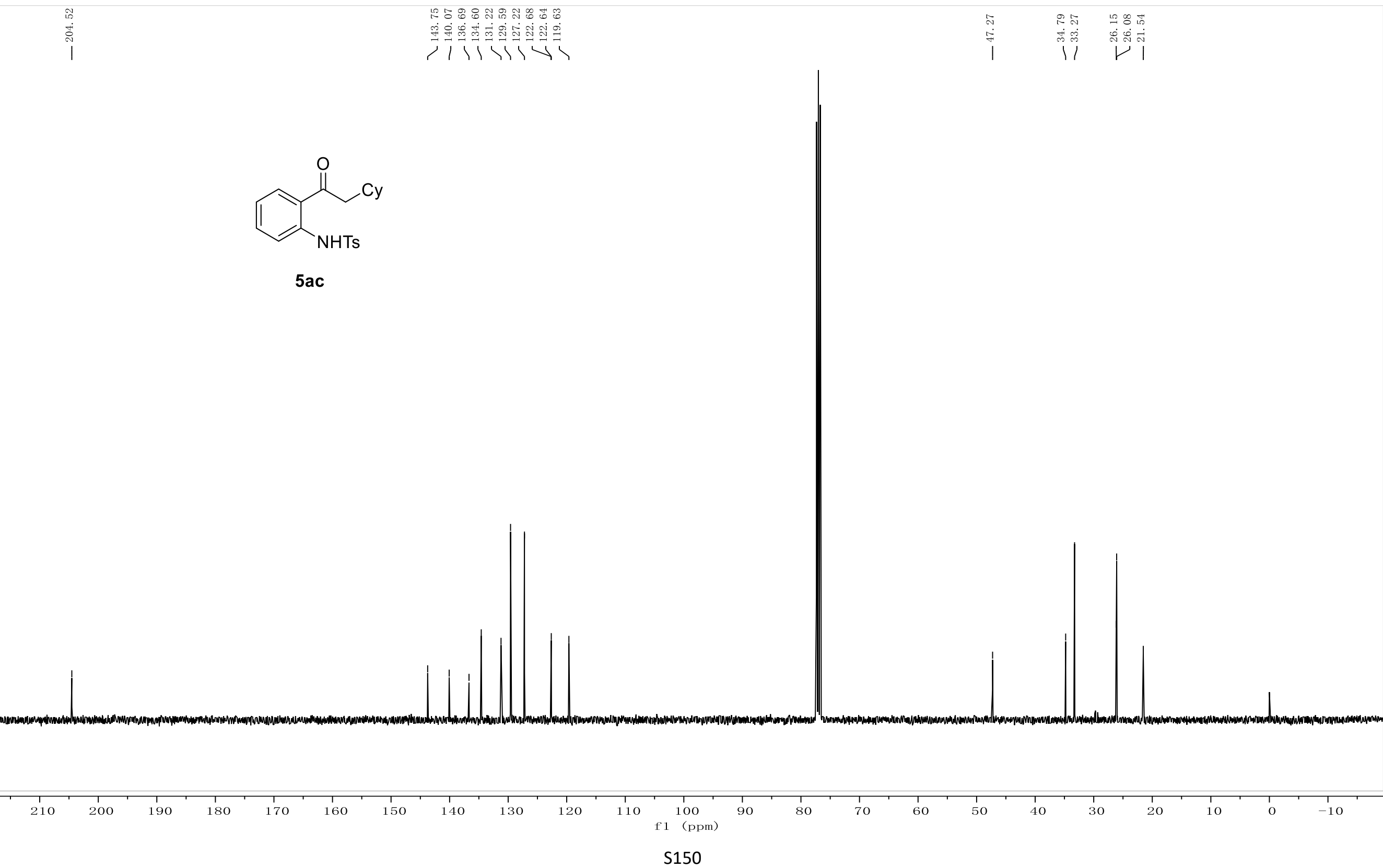
${ }^{1} \mathrm{H}$ NMR-spectrum $\left(500 \mathrm{MHz}, \mathrm{CDCl}_{3}\right)$ of $\mathbf{5 a i}$

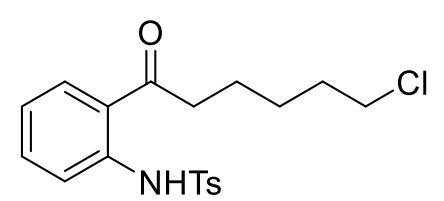

5ai

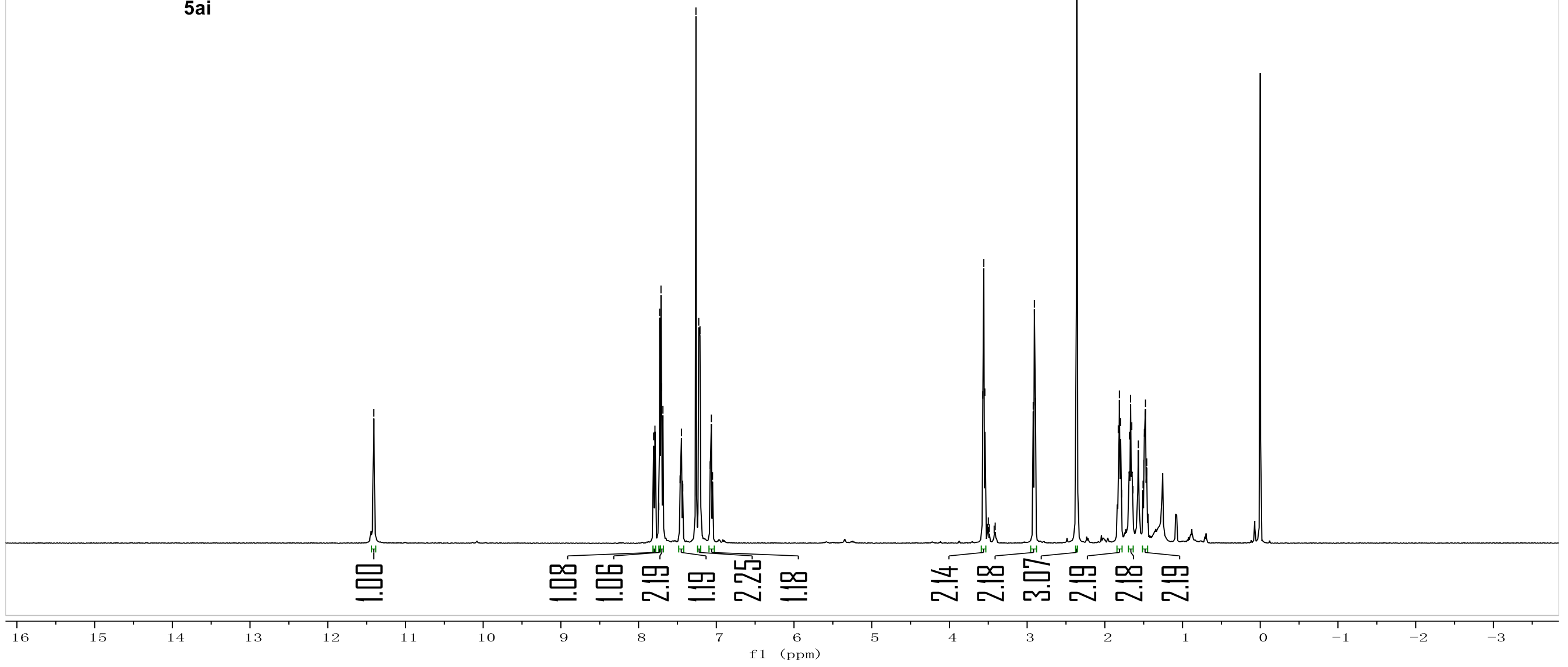


${ }^{13} \mathrm{CNMR}$-spectrum $\left(126 \mathrm{MHz}, \mathrm{CDCl}_{3}\right)$ of $\mathbf{5 a i}$
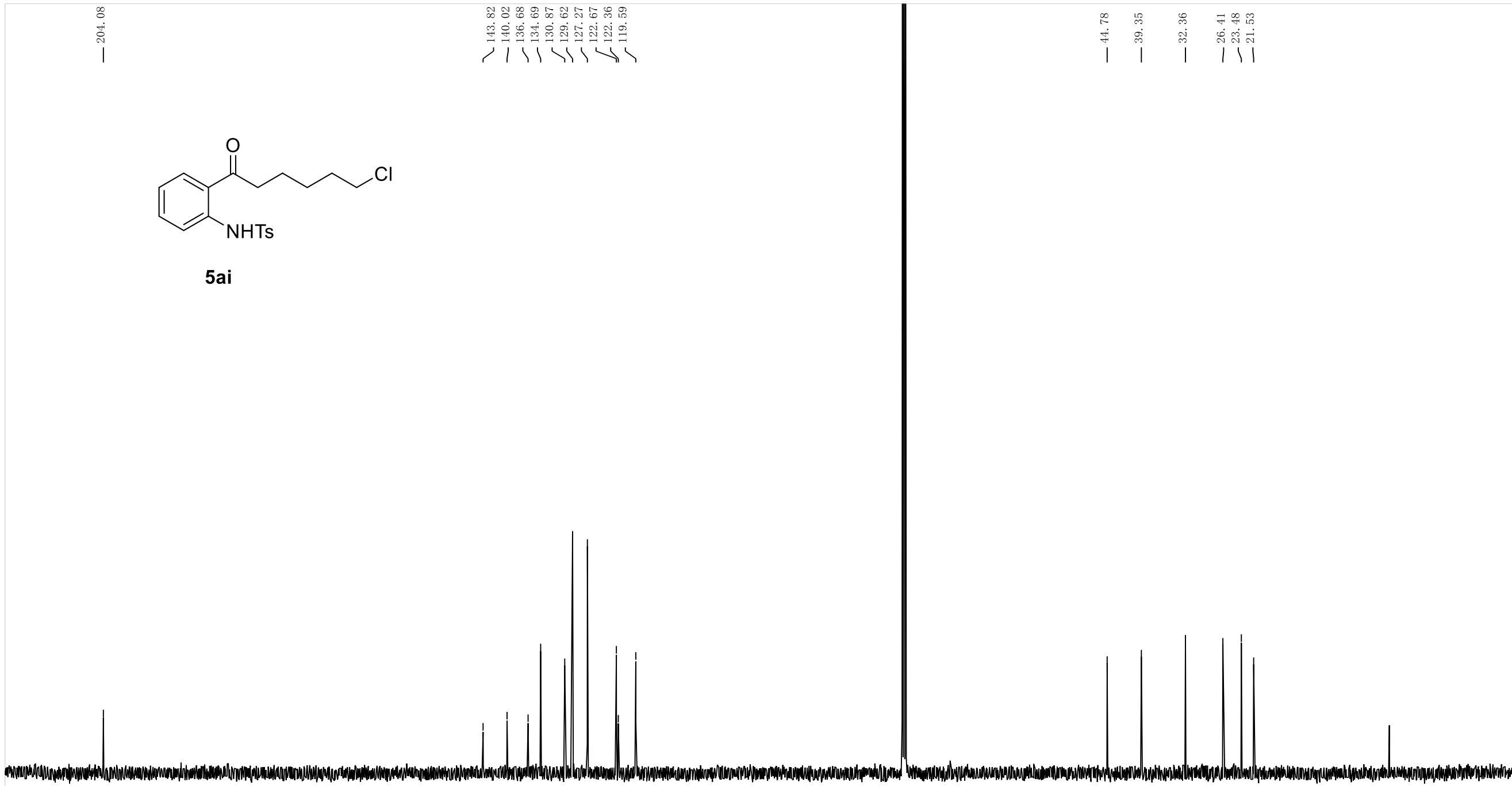

$210 \quad 200 \quad 190$ 
${ }^{1} \mathrm{H}$ NMR-spectrum $\left(400 \mathrm{MHz}, \mathrm{CDCl}_{3}\right)$ of $\mathbf{5 a k}$

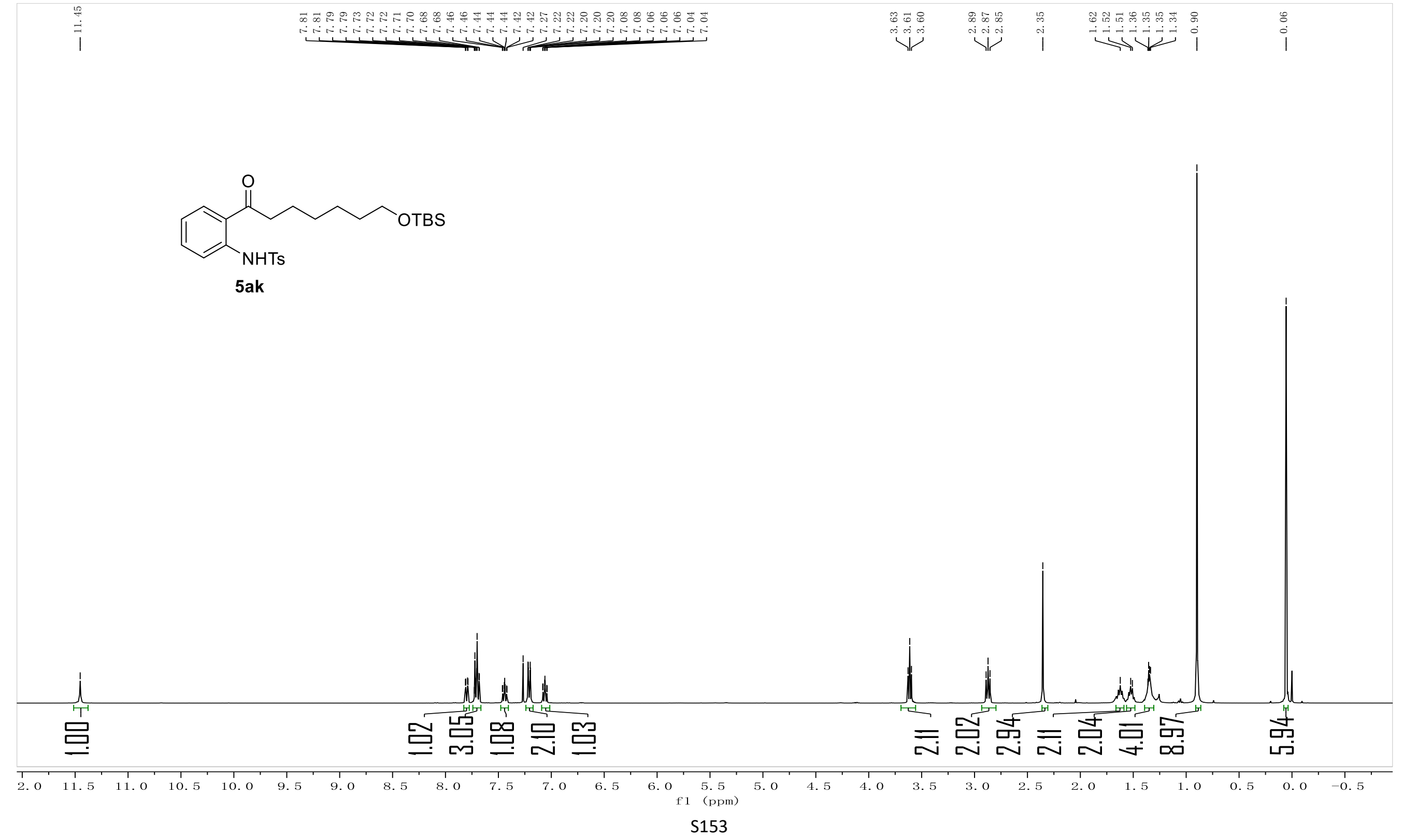


${ }^{13} \mathrm{CNMR}$-spectrum $\left(101 \mathrm{MHz}, \mathrm{CDCl}_{3}\right)$ of $\mathbf{5 a k}$
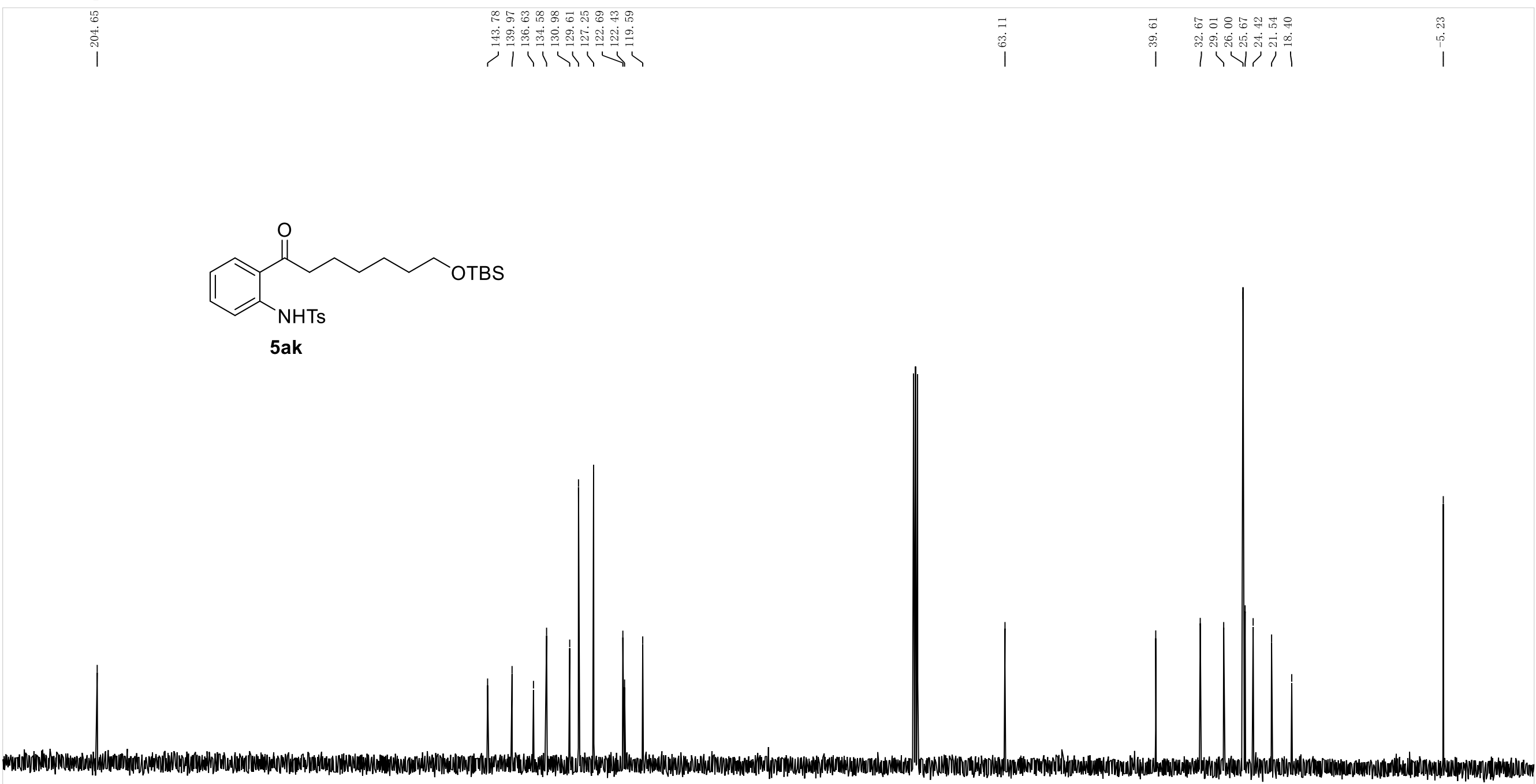

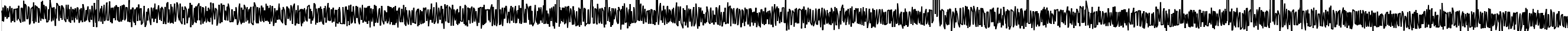

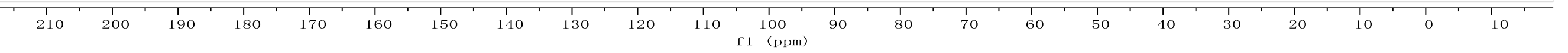


${ }^{1} \mathrm{H}$ NMR-spectrum $\left(400 \mathrm{MHz}, \mathrm{CDCl}_{3}\right)$ of $\mathbf{5 a l}$

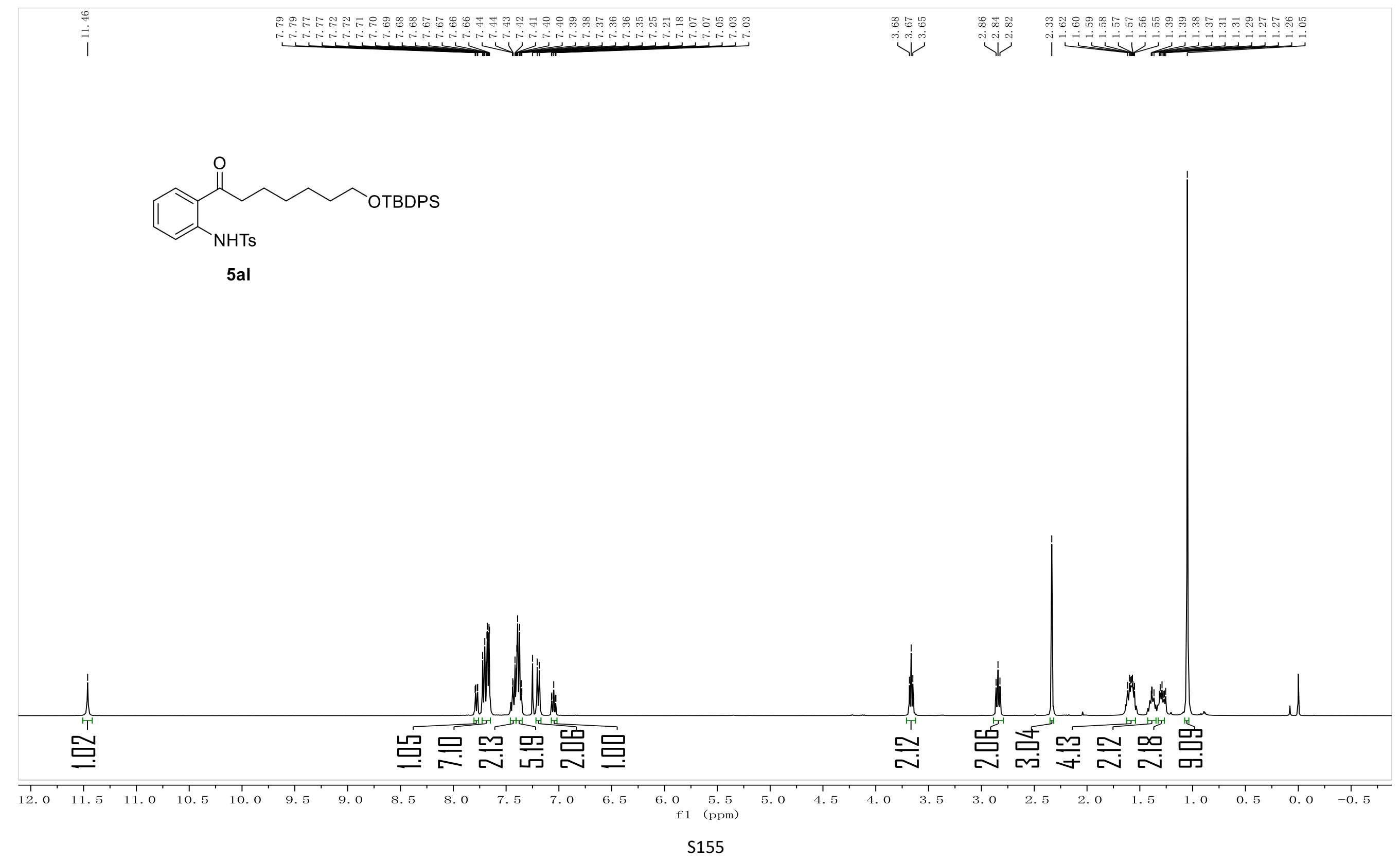


${ }^{13} \mathrm{CNMR}$-spectrum $\left(101 \mathrm{MHz}, \mathrm{CDCl}_{3}\right)$ of $\mathbf{5 a l}$
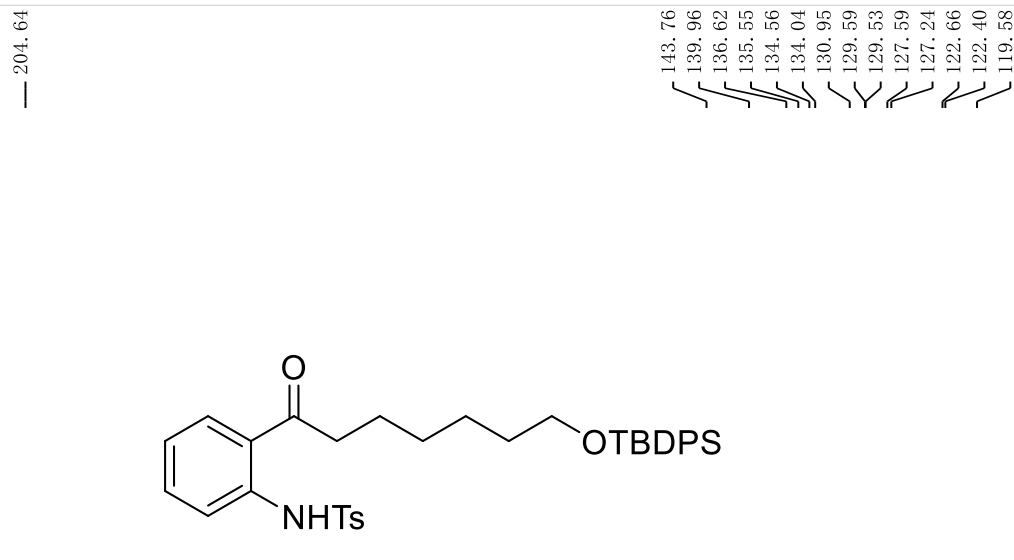

5al

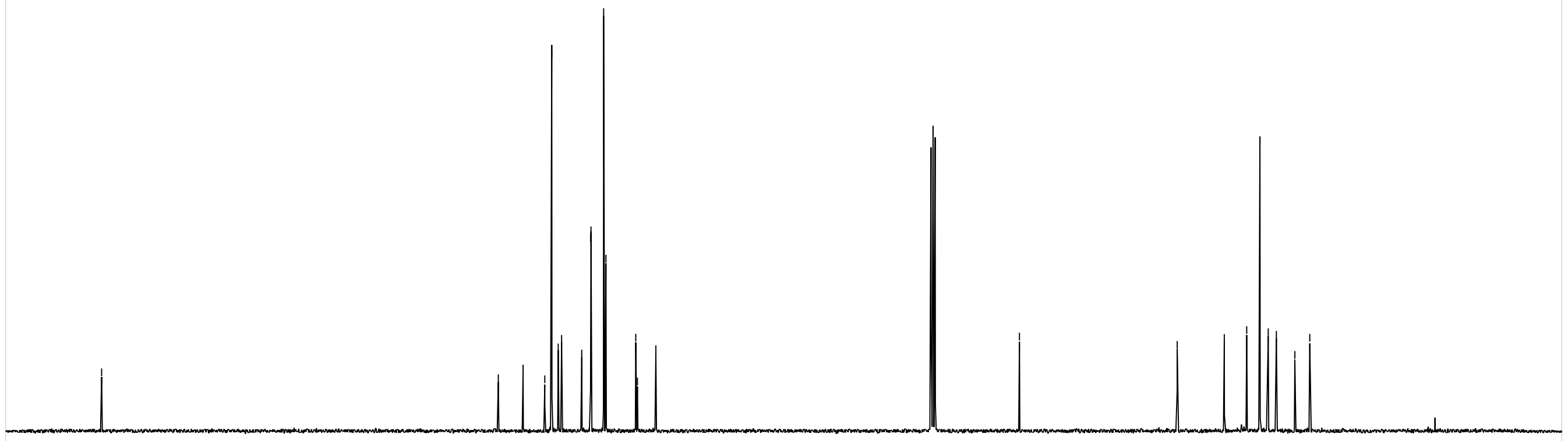

$210 \quad 200$
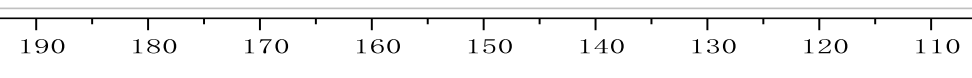

100
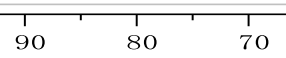

60

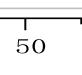

40

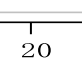

10 
${ }^{1} \mathrm{H}$ NMR-spectrum $\left(500 \mathrm{MHz}, \mathrm{CDCl}_{3}\right)$ of $\mathbf{5 a q}$

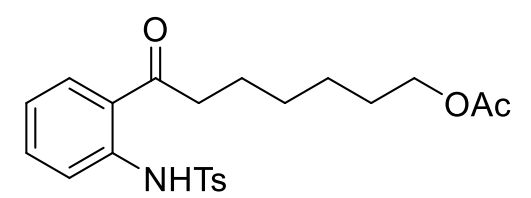

$5 \mathrm{aq}$

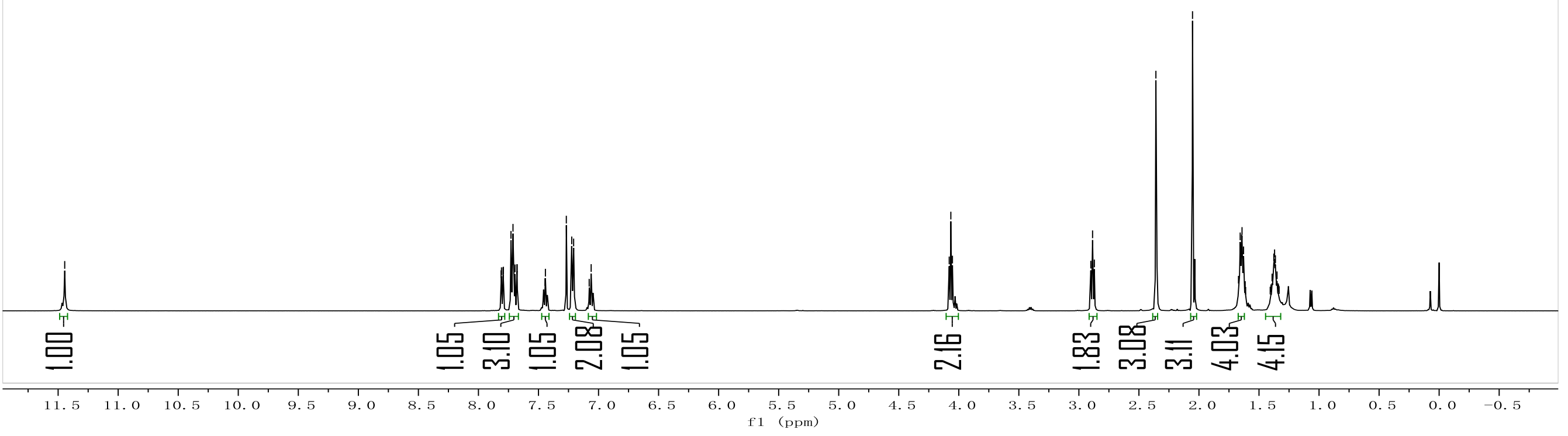


${ }^{13} \mathrm{CNMR}$-spectrum $\left(126 \mathrm{MHz}, \mathrm{CDCl}_{3}\right)$ of $\mathbf{5 a q}$

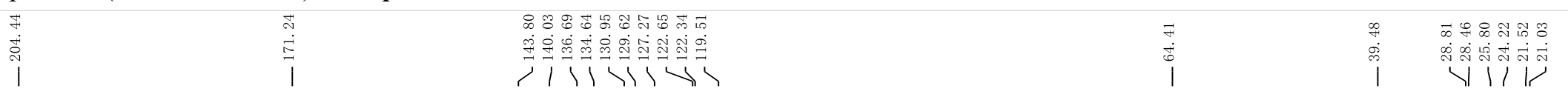

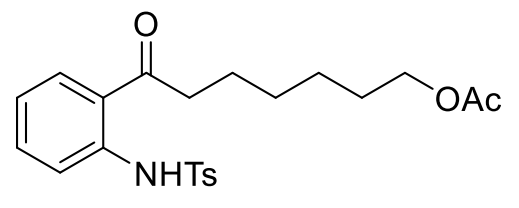

5 aq

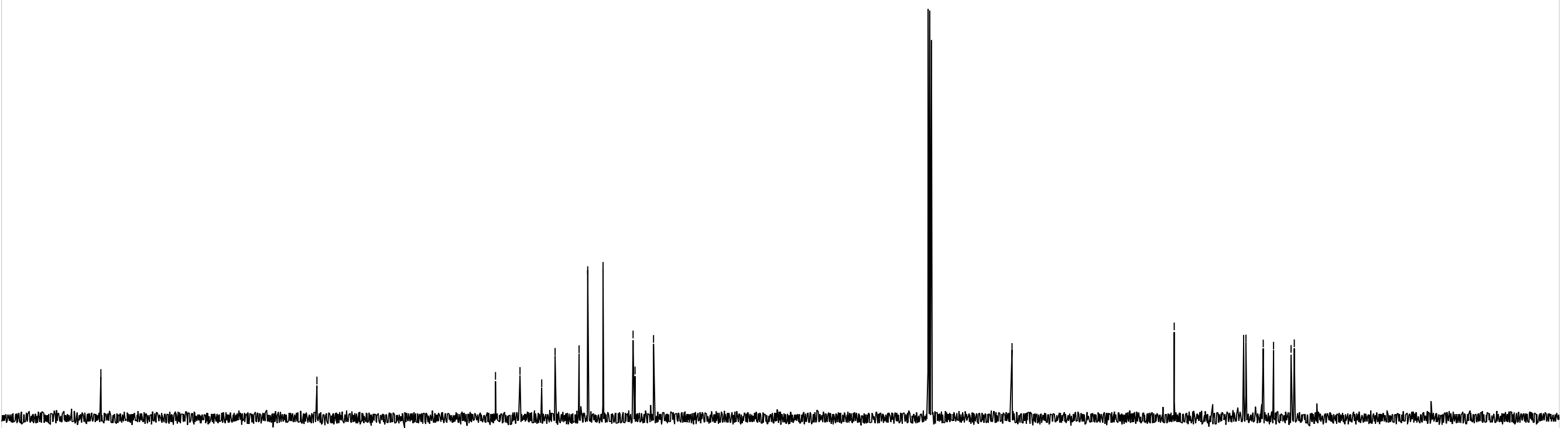

$210+200+190$

$180 \quad 170$

$60 \quad 150$

140

$30 \quad 120$ 


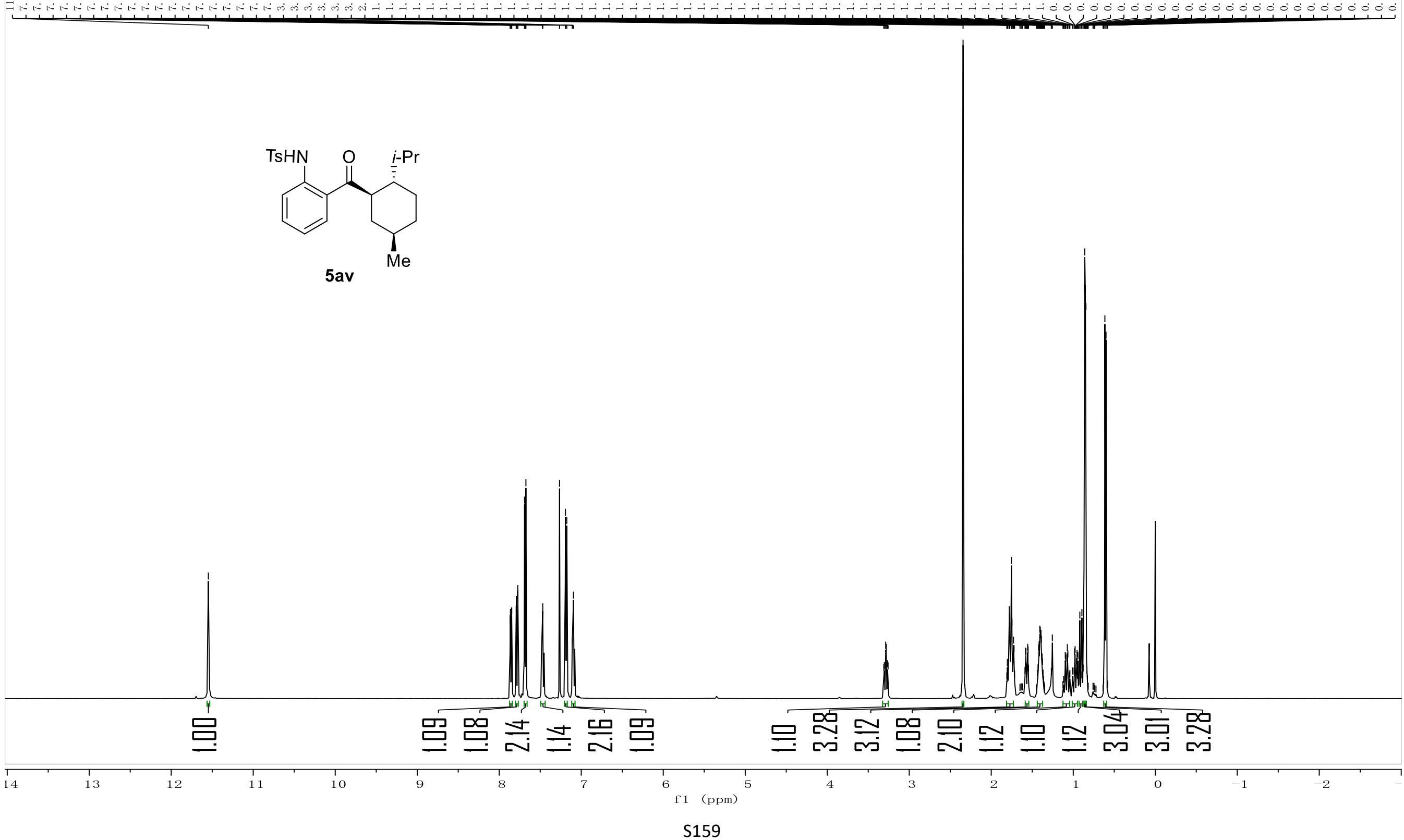


${ }^{13} \mathrm{CNMR}$-spectrum $\left(126 \mathrm{MHz}, \mathrm{CDCl}_{3}\right)$ of $\mathbf{5 a v}$
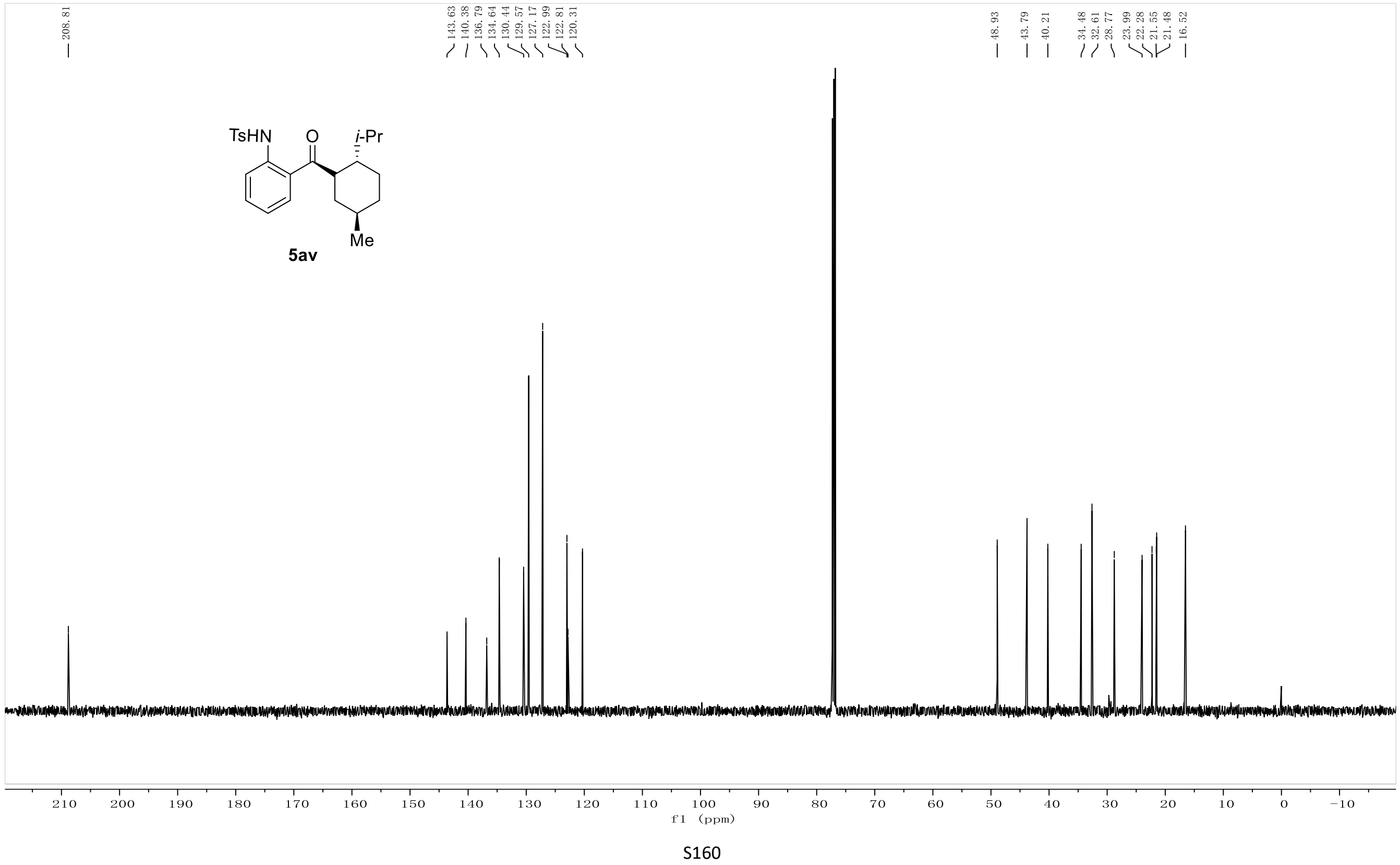
COSY-spectrum of $\mathbf{5 a v}$

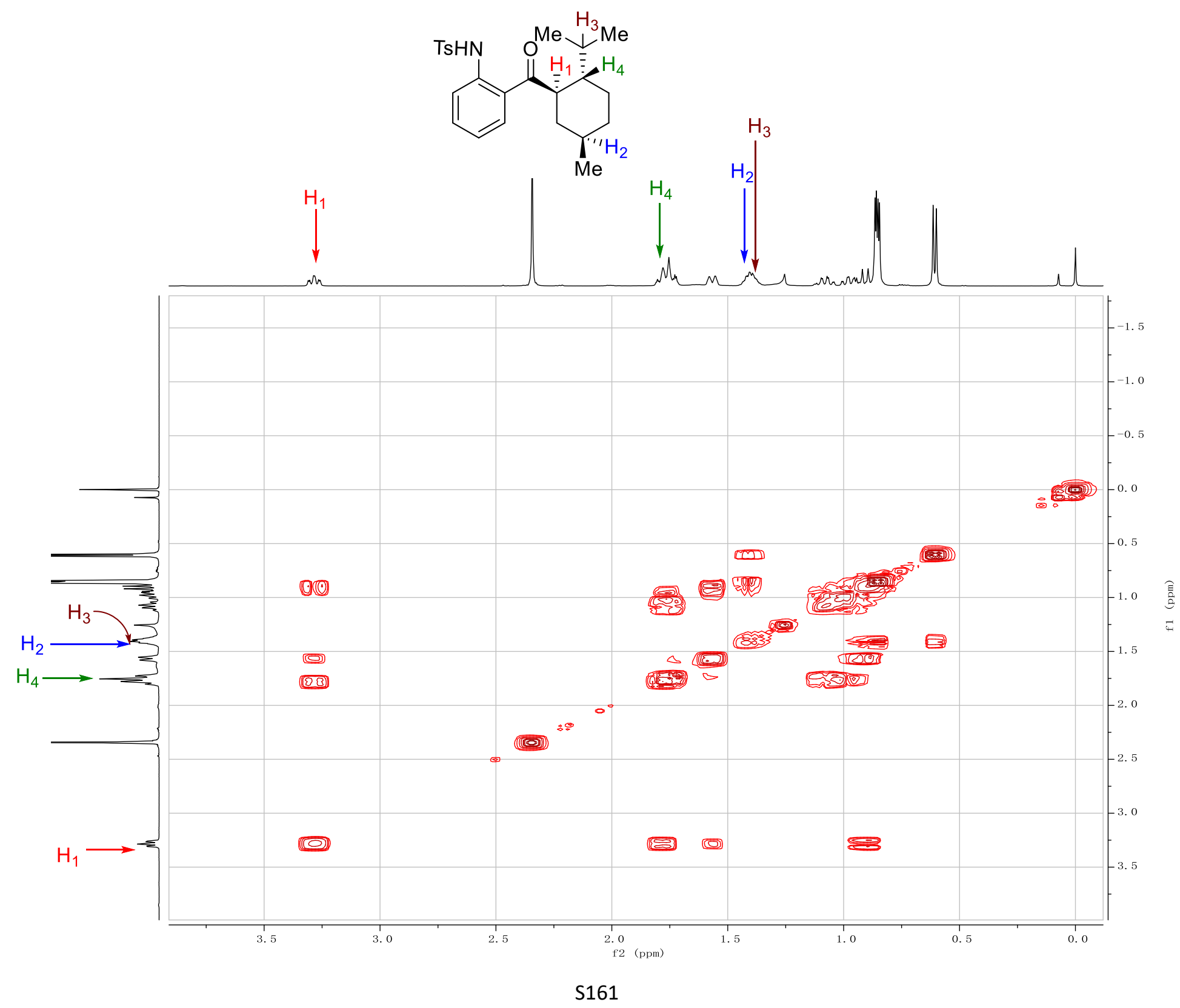


DEPT- $135^{\circ}$-spectrum of $\mathbf{5 a v}$

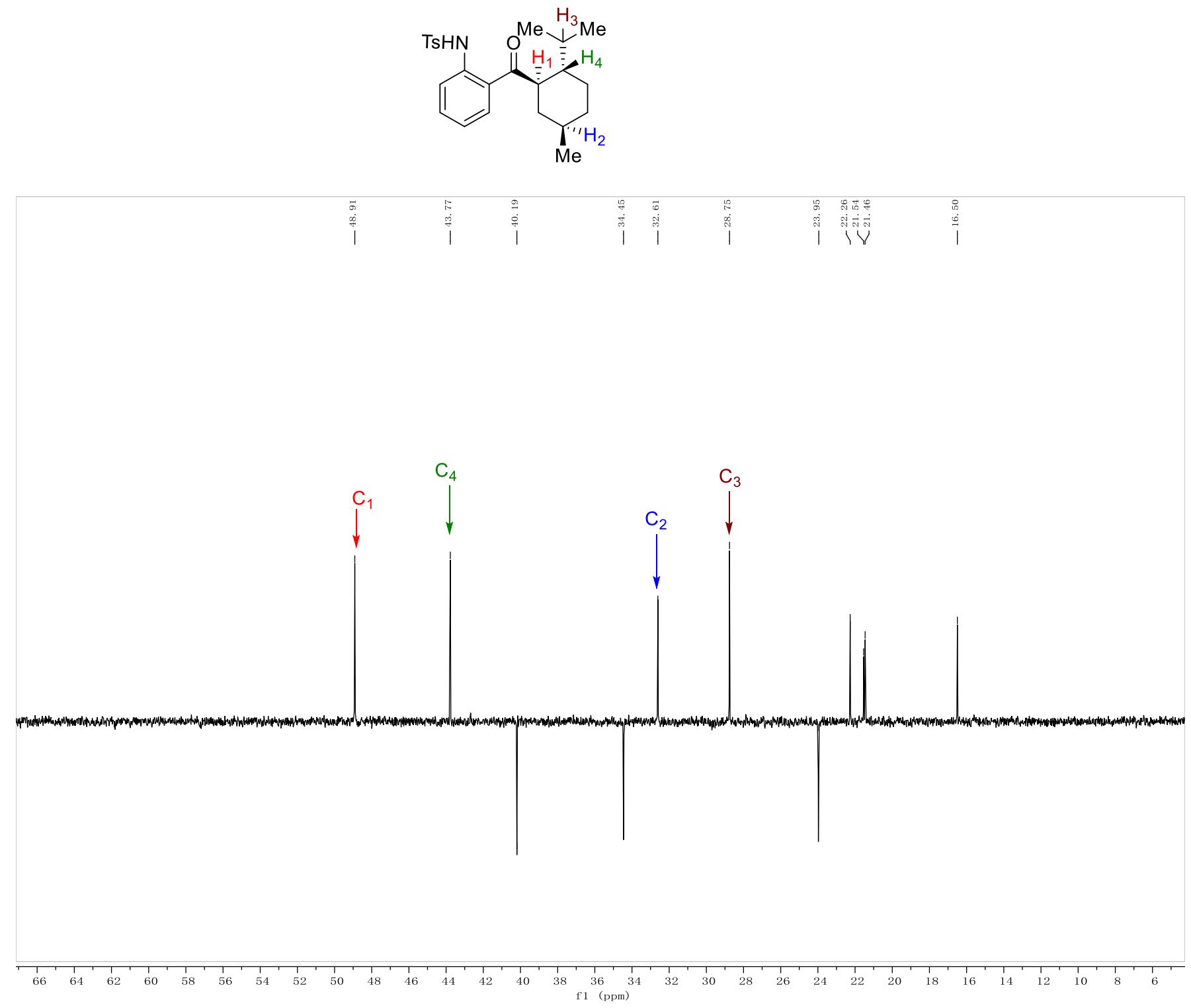


HSQC-spectrum of $\mathbf{5 a v}$

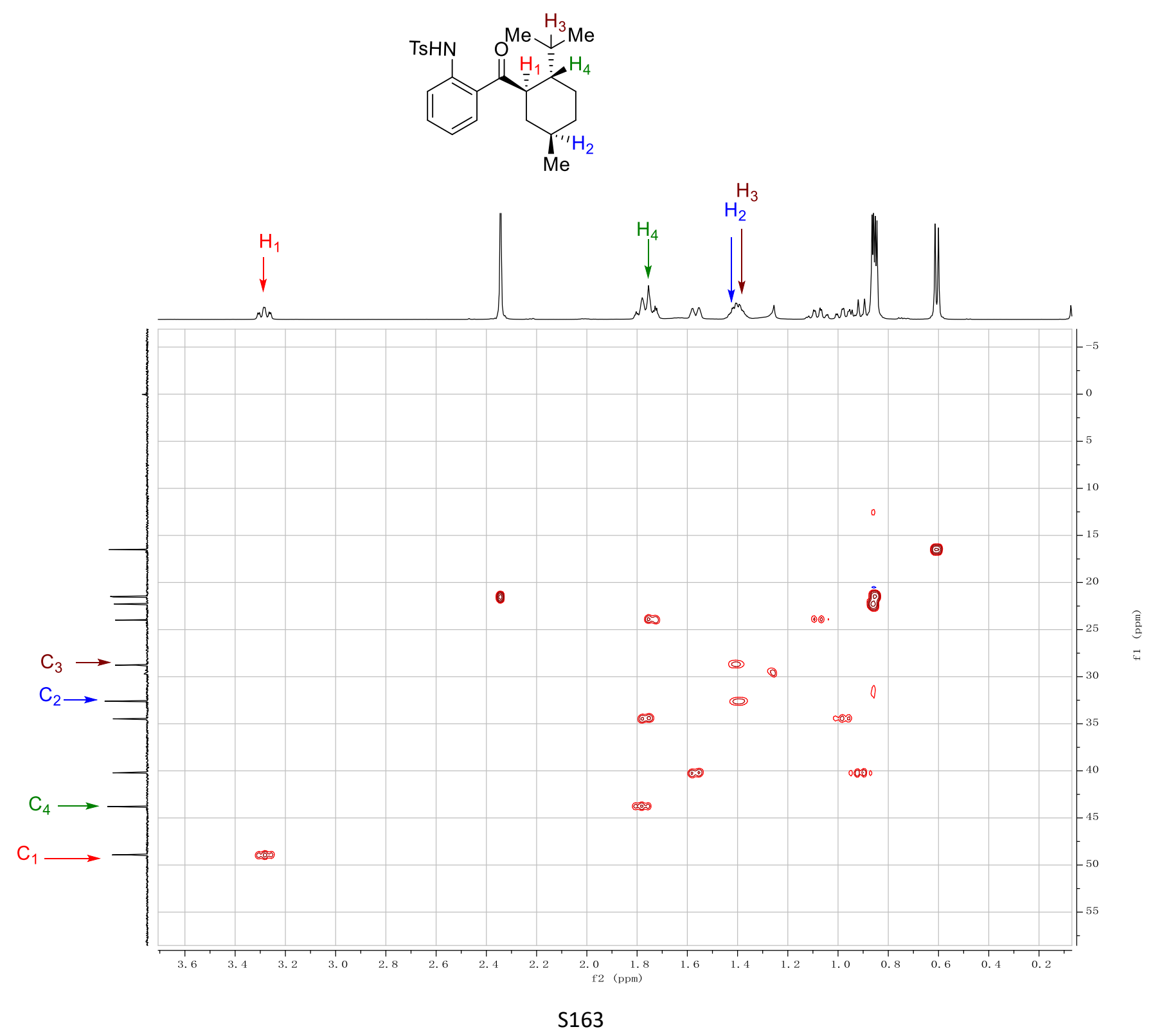




\section{NOSEY-spectrum of $\mathbf{5 a v}$}
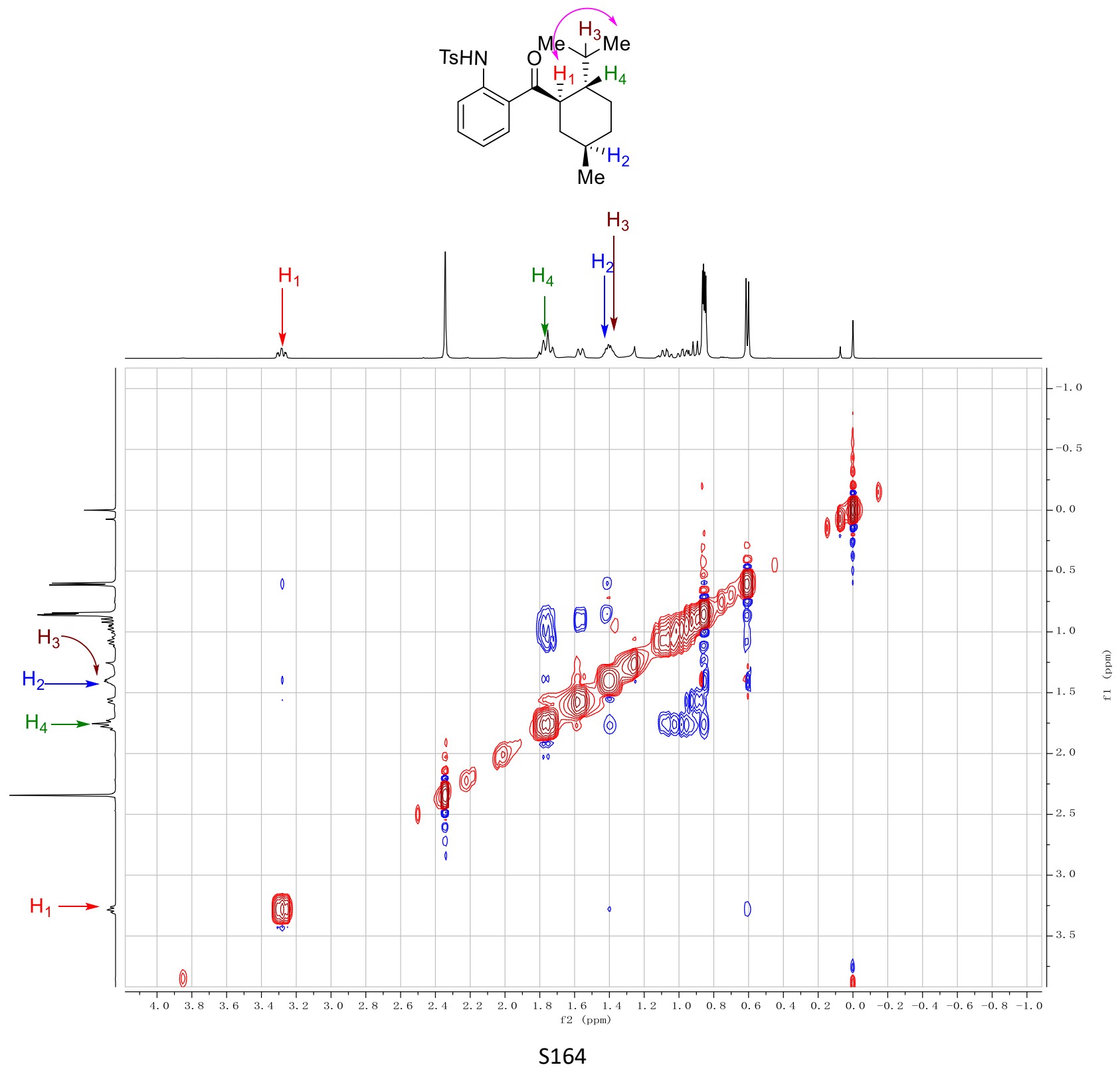
${ }^{1} \mathrm{H}$ NMR-spectrum $\left(500 \mathrm{MHz}, \mathrm{CDCl}_{3}\right)$ of $\mathbf{5 a w}$

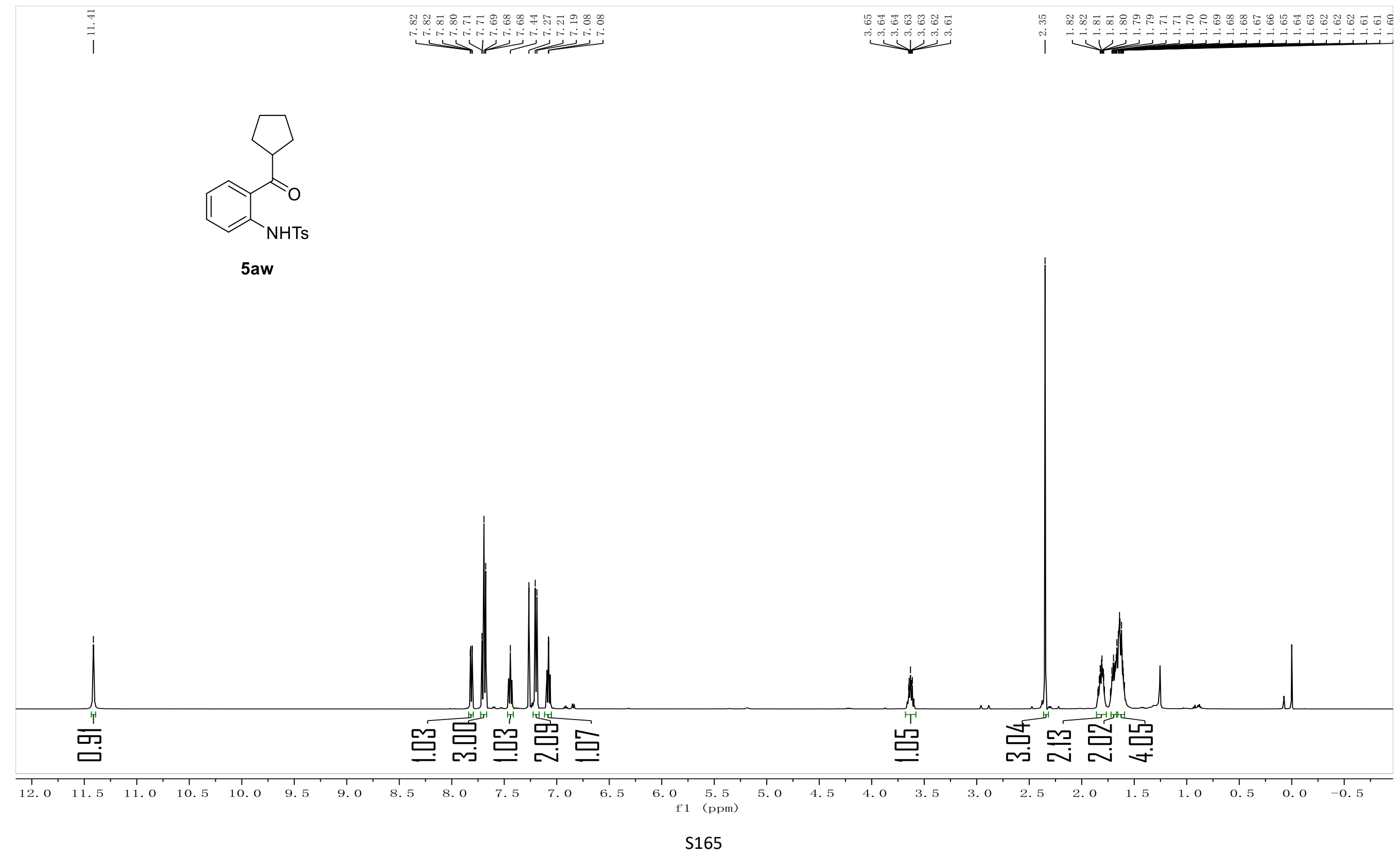


${ }^{13} \mathrm{C} \mathrm{NMR}$-spectrum $\left(126 \mathrm{MHz}, \mathrm{CDCl}_{3}\right)$ of $\mathbf{5 a w}$

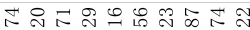

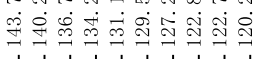

|रेरों

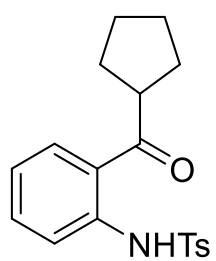

5aw

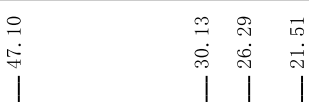

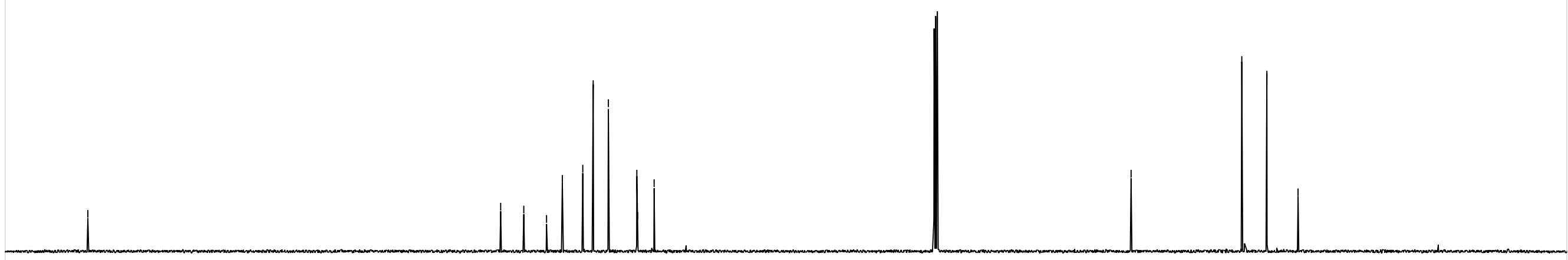

210
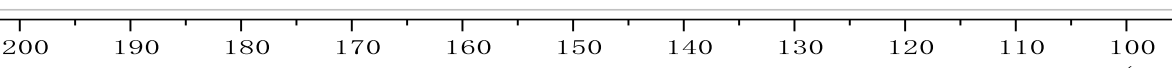

100
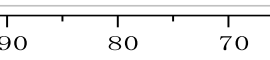
${ }^{1} \mathrm{H}$ NMR-spectrum $\left(500 \mathrm{MHz}, \mathrm{CDCl}_{3}\right)$ of $\mathbf{5 a x}$

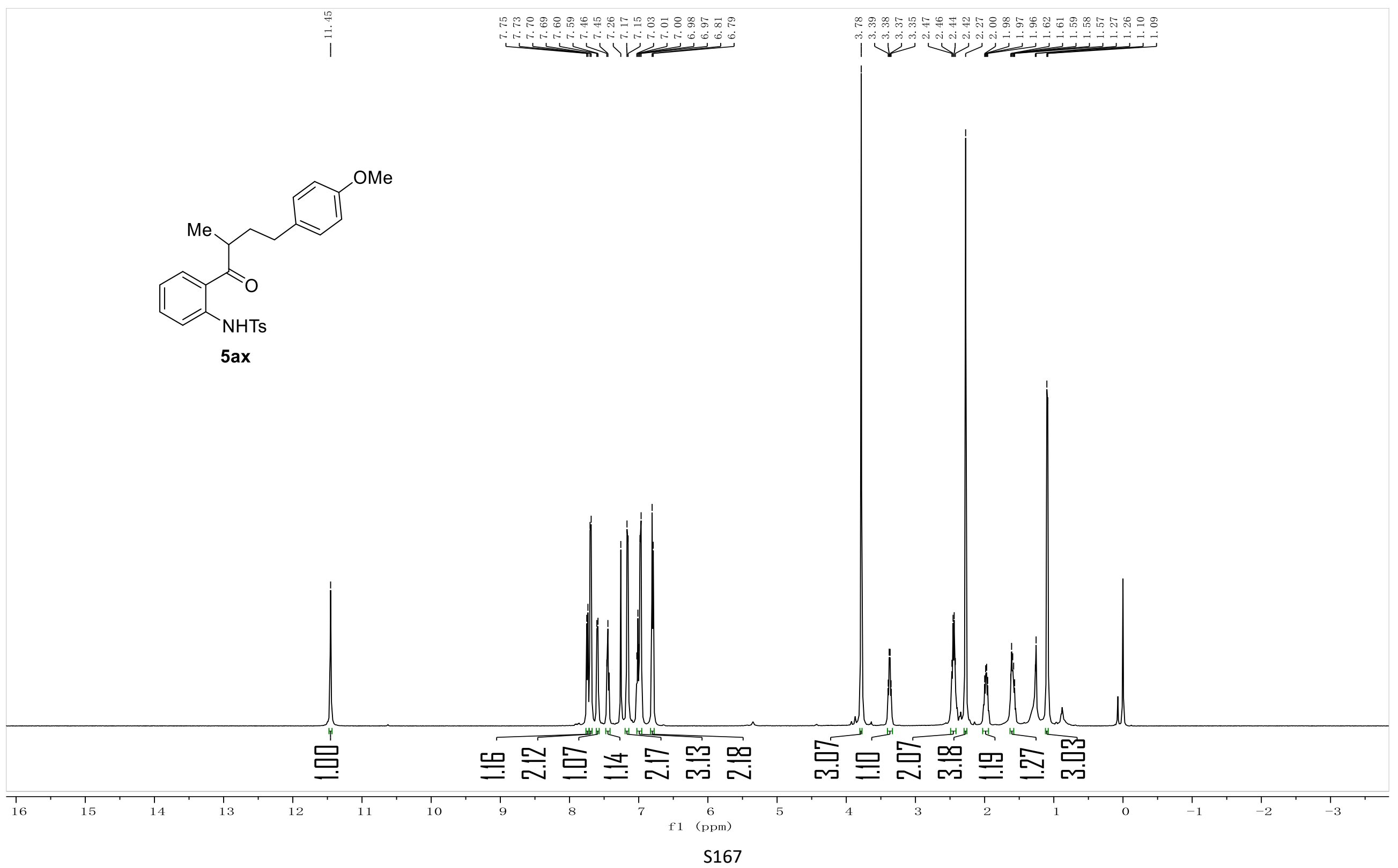


${ }^{13} \mathrm{C}$ NMR-spectrum $\left(126 \mathrm{MHz}, \mathrm{CDCl}_{3}\right)$ of $\mathbf{5 a x}$

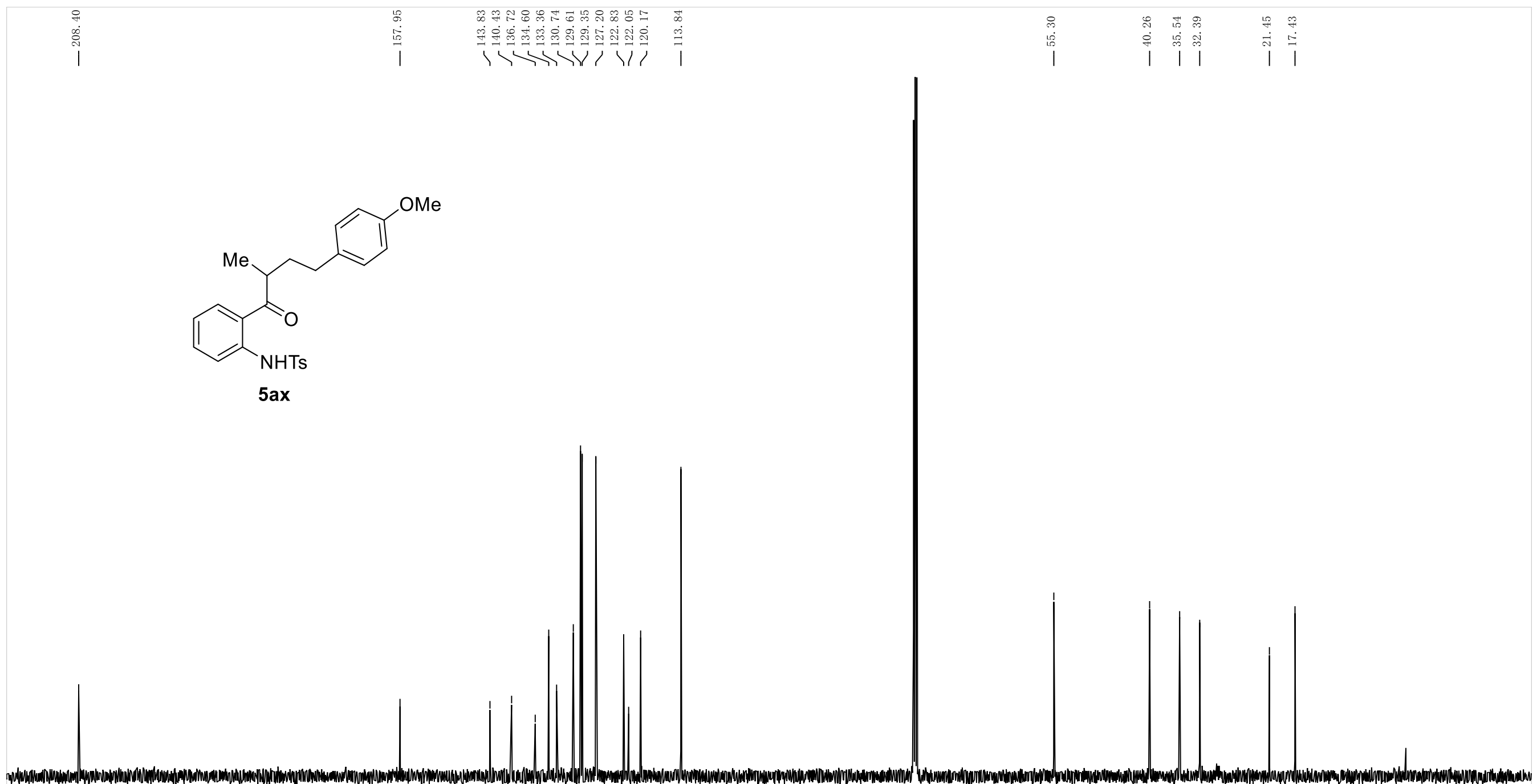

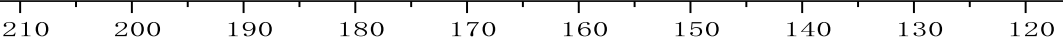
110 f1 100
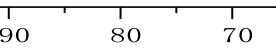
60

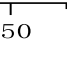
40 30 20 10 
${ }^{1} \mathrm{H}$ NMR-spectrum $\left(400 \mathrm{MHz}, \mathrm{CDCl}_{3}\right)$ of $\mathbf{5} \mathbf{b k}$

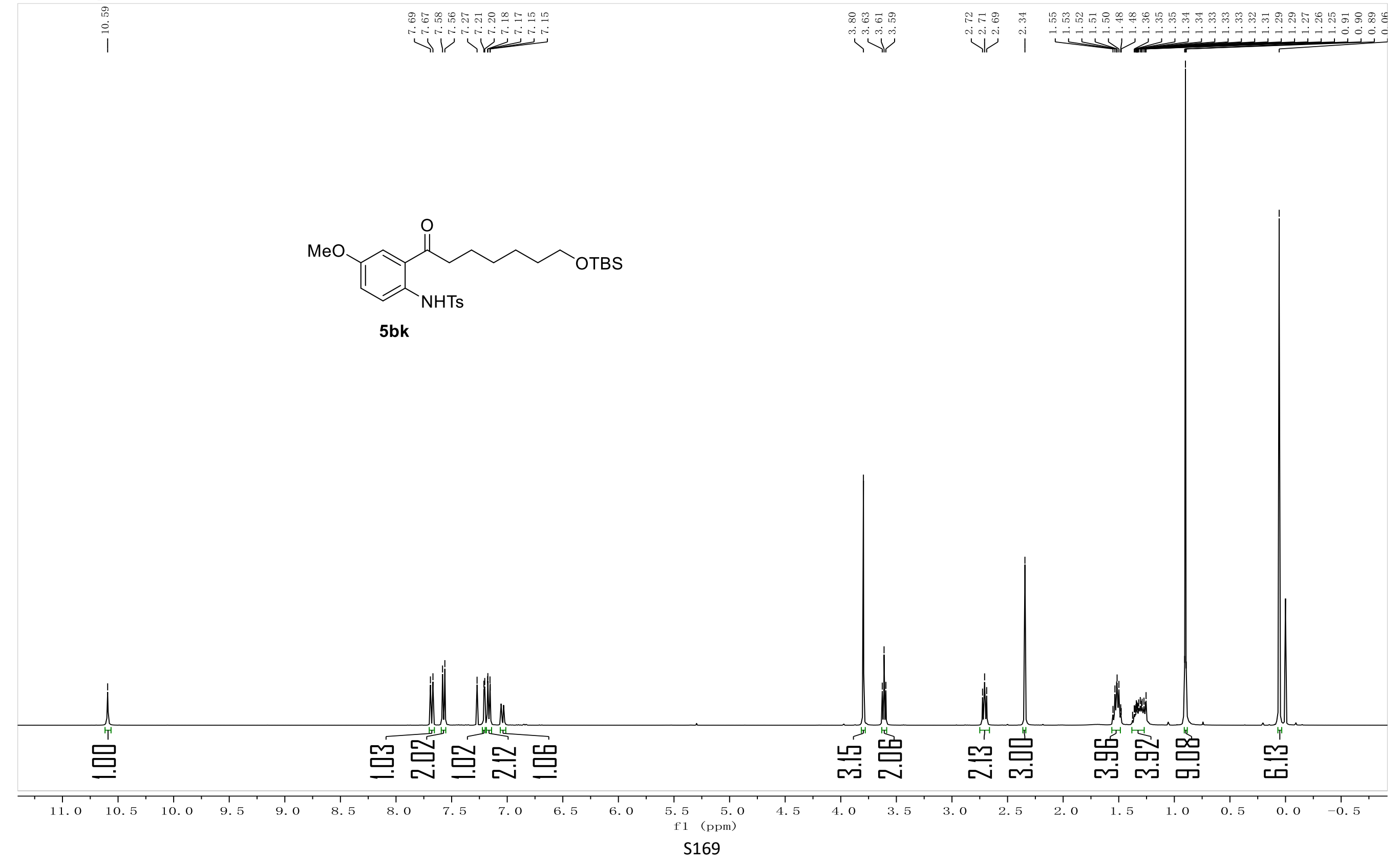


${ }^{13} \mathrm{C} \mathrm{NMR}$-spectrum $\left(101 \mathrm{MHz}, \mathrm{CDCl}_{3}\right)$ of $\mathbf{5} \mathbf{b k}$

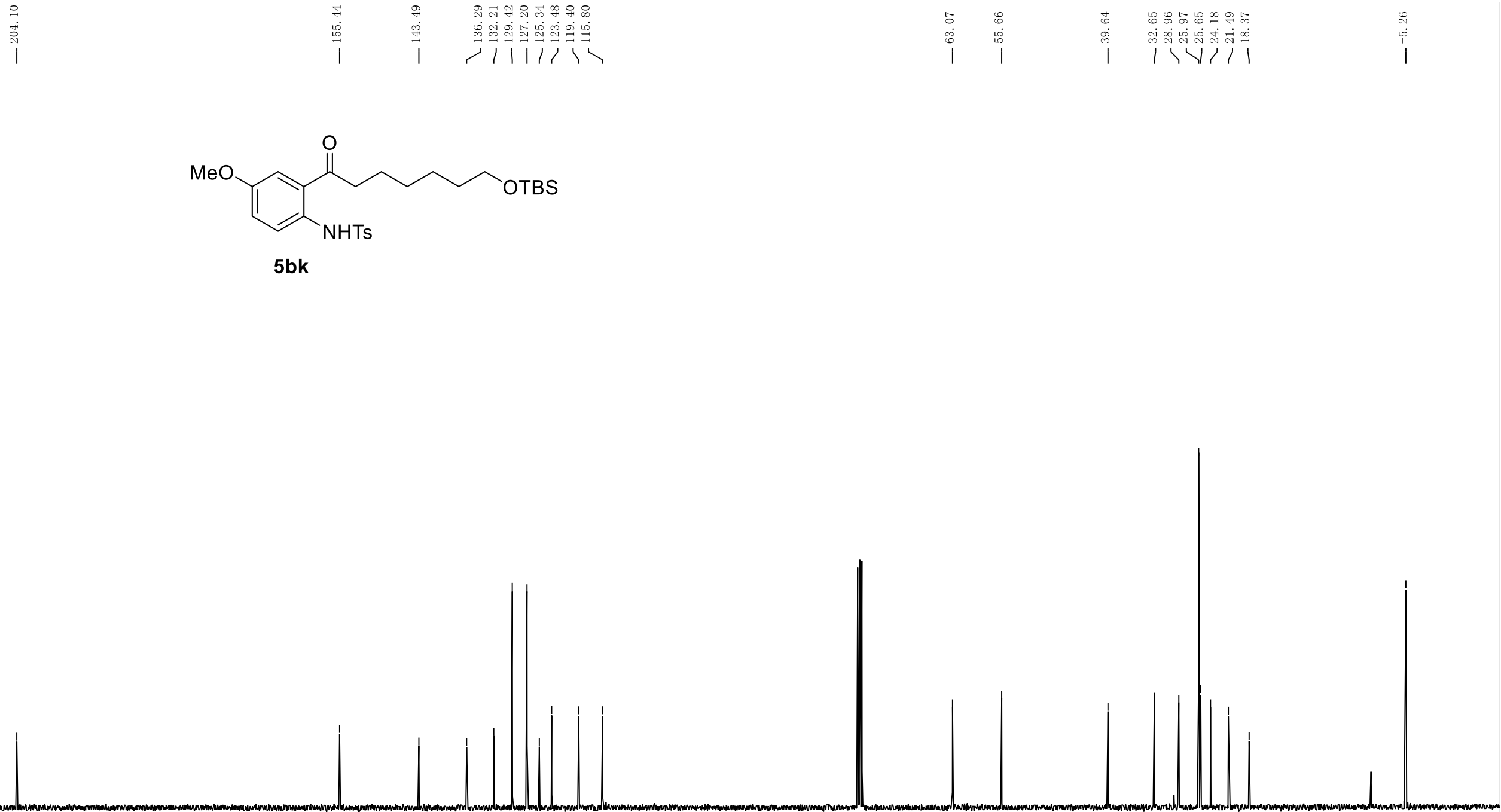

210

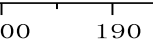

180

$170 \quad 160$

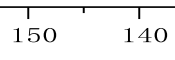

130
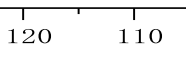

100

1
$90 \quad 80$

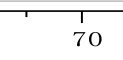

60
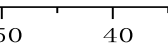

30 


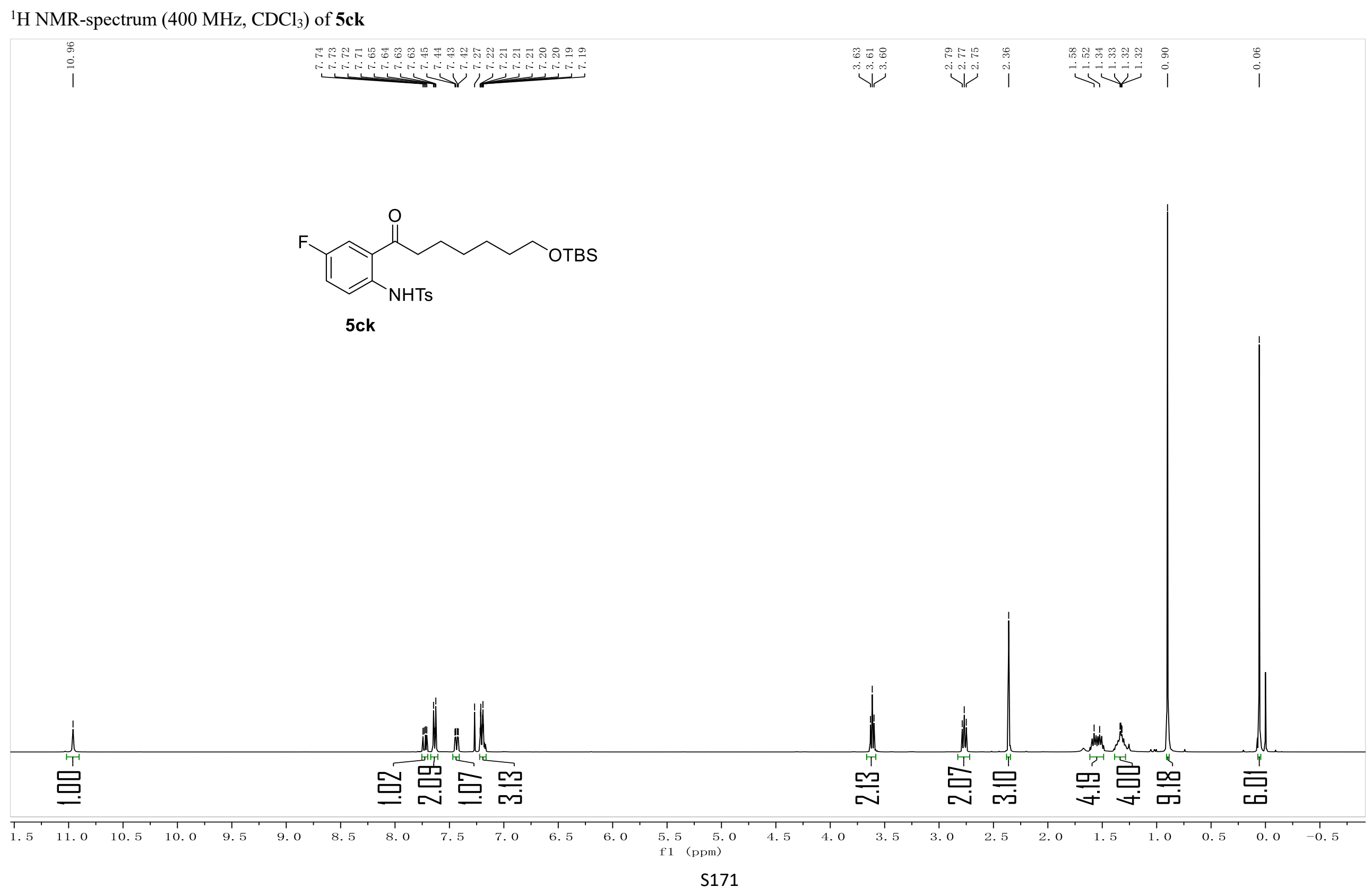


${ }^{13} \mathrm{C}$ NMR-spectrum $\left(101 \mathrm{MHz}, \mathrm{CDCl}_{3}\right)$ of $\mathbf{5} \mathbf{k}$
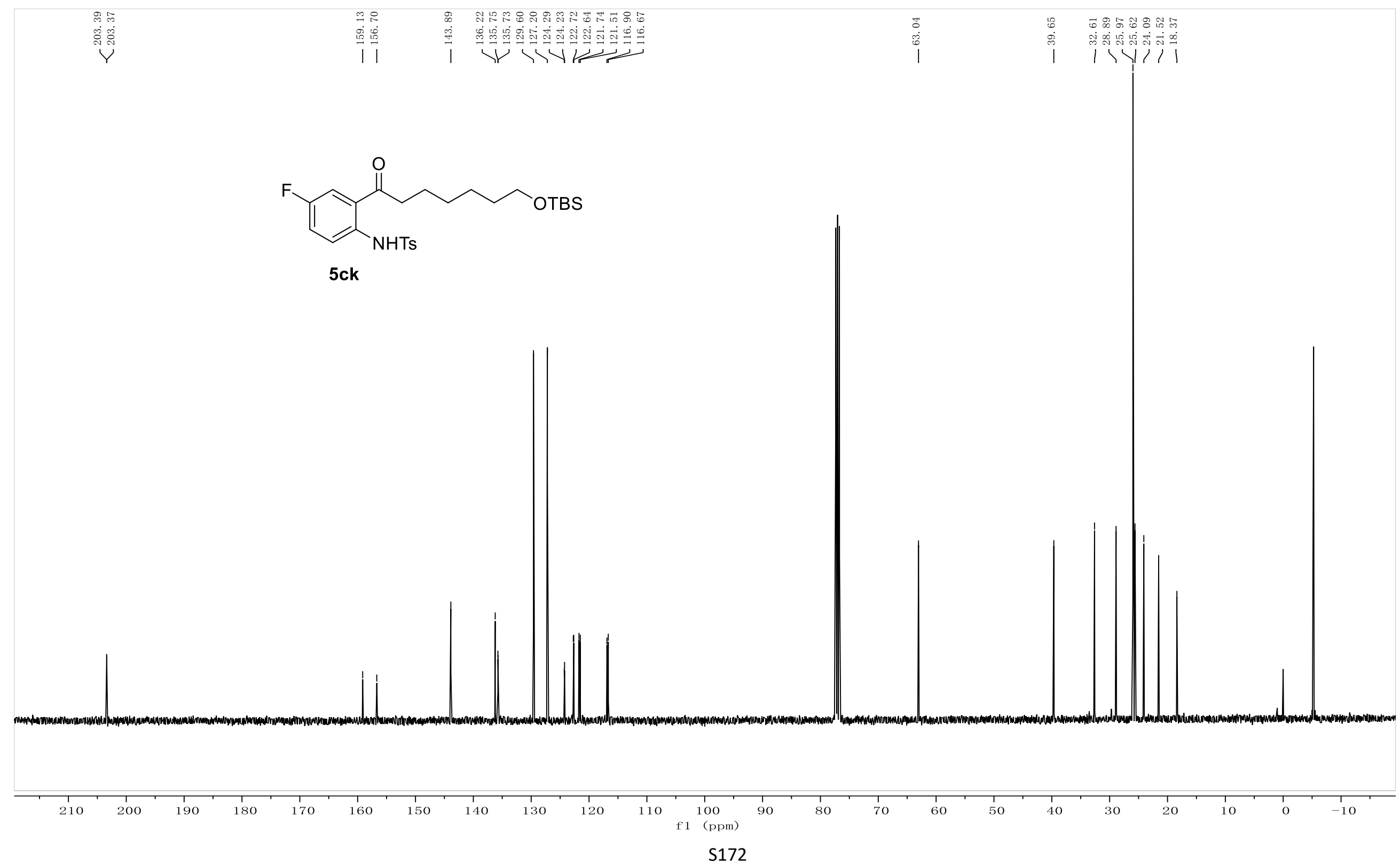
${ }^{19} \mathrm{~F}$ NMR-spectrum $\left(376 \mathrm{MHz}, \mathrm{CDCl}_{3}\right)$ of $\mathbf{5} \mathbf{c k}$

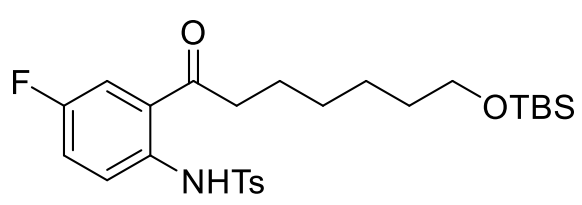

$5 \mathrm{ck}$
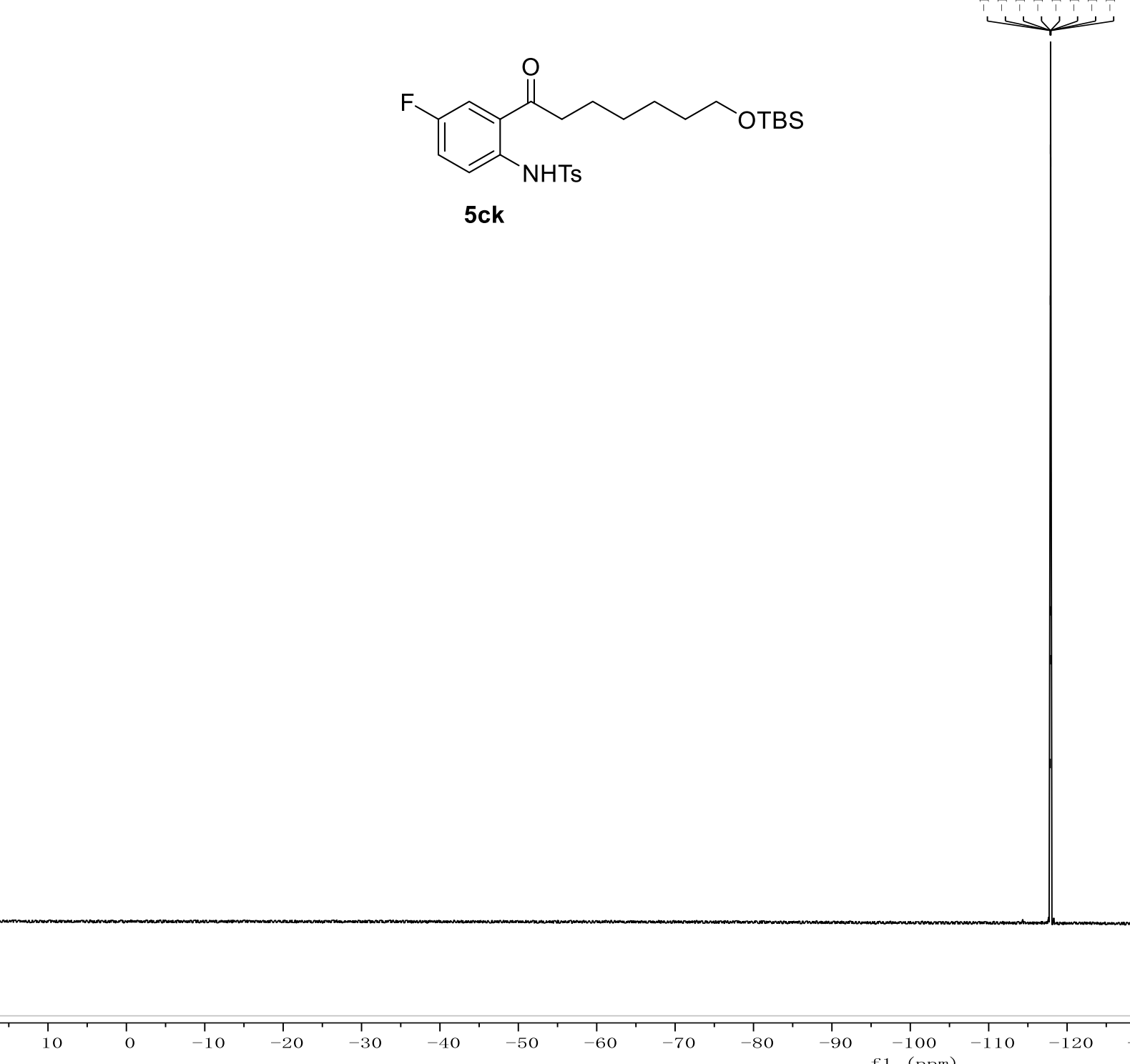
${ }^{1} \mathrm{H}$ NMR-spectrum $\left(400 \mathrm{MHz}, \mathrm{CDCl}_{3}\right)$ of $\mathbf{5} \mathbf{d k}$

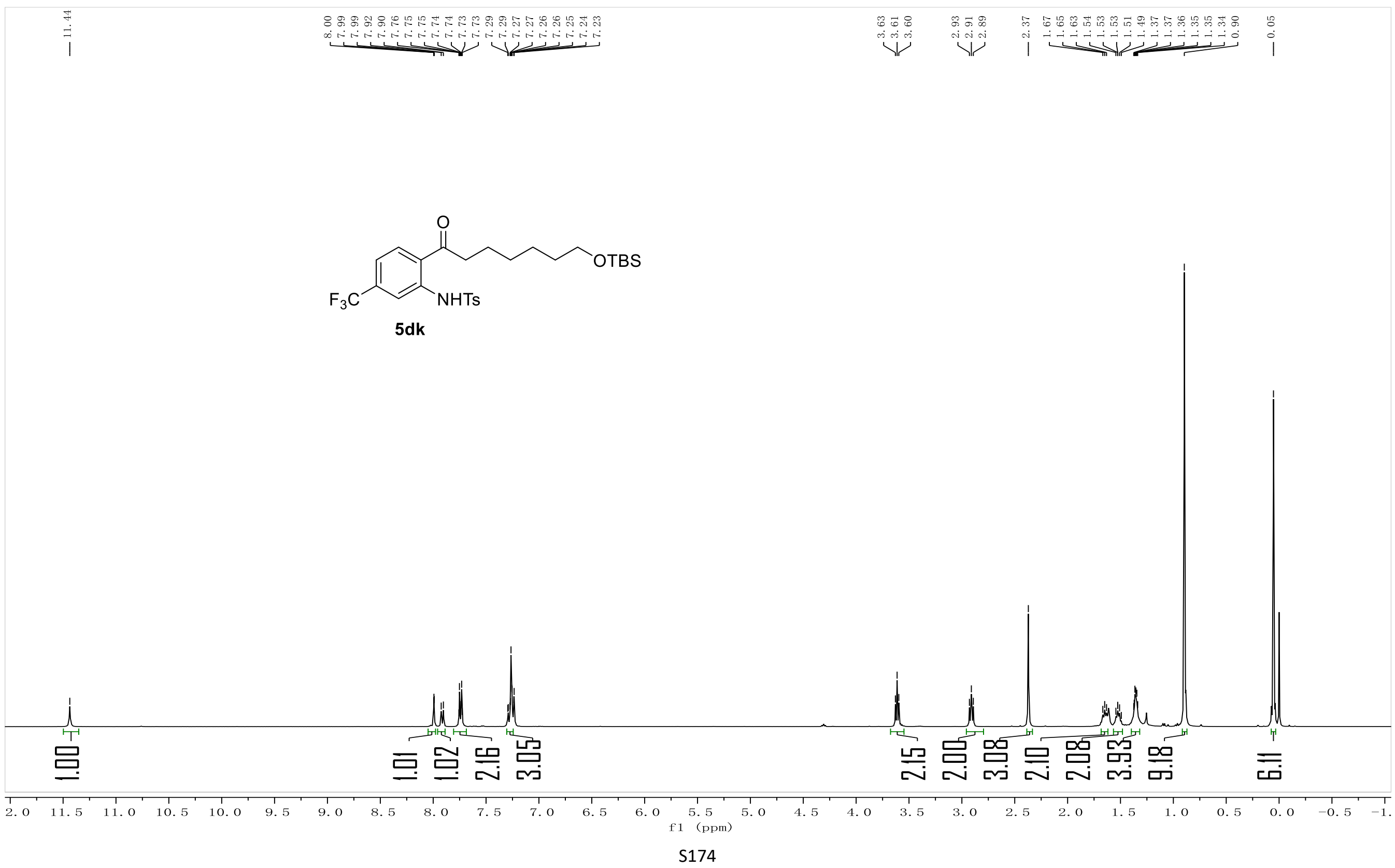


${ }^{13} \mathrm{C}$ NMR-spectrum $\left(101 \mathrm{MHz}, \mathrm{CDCl}_{3}\right)$ of $\mathbf{5 d k}$

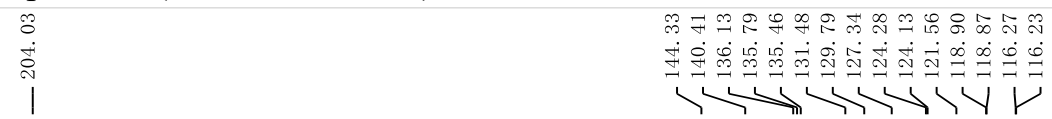
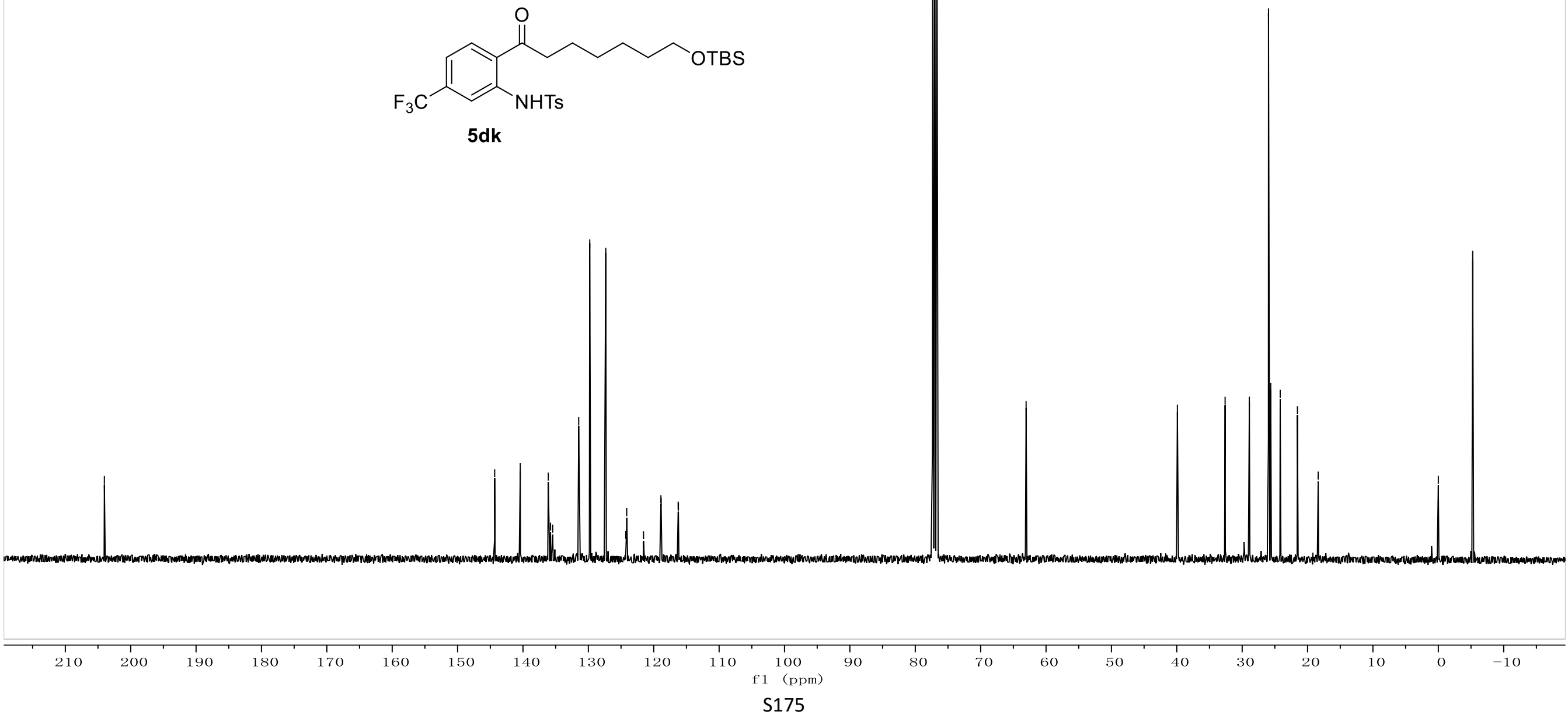
${ }^{19} \mathrm{~F}$ NMR-spectrum $\left(376 \mathrm{MHz}, \mathrm{CDCl}_{3}\right)$ of $\mathbf{5} \mathbf{d k}$
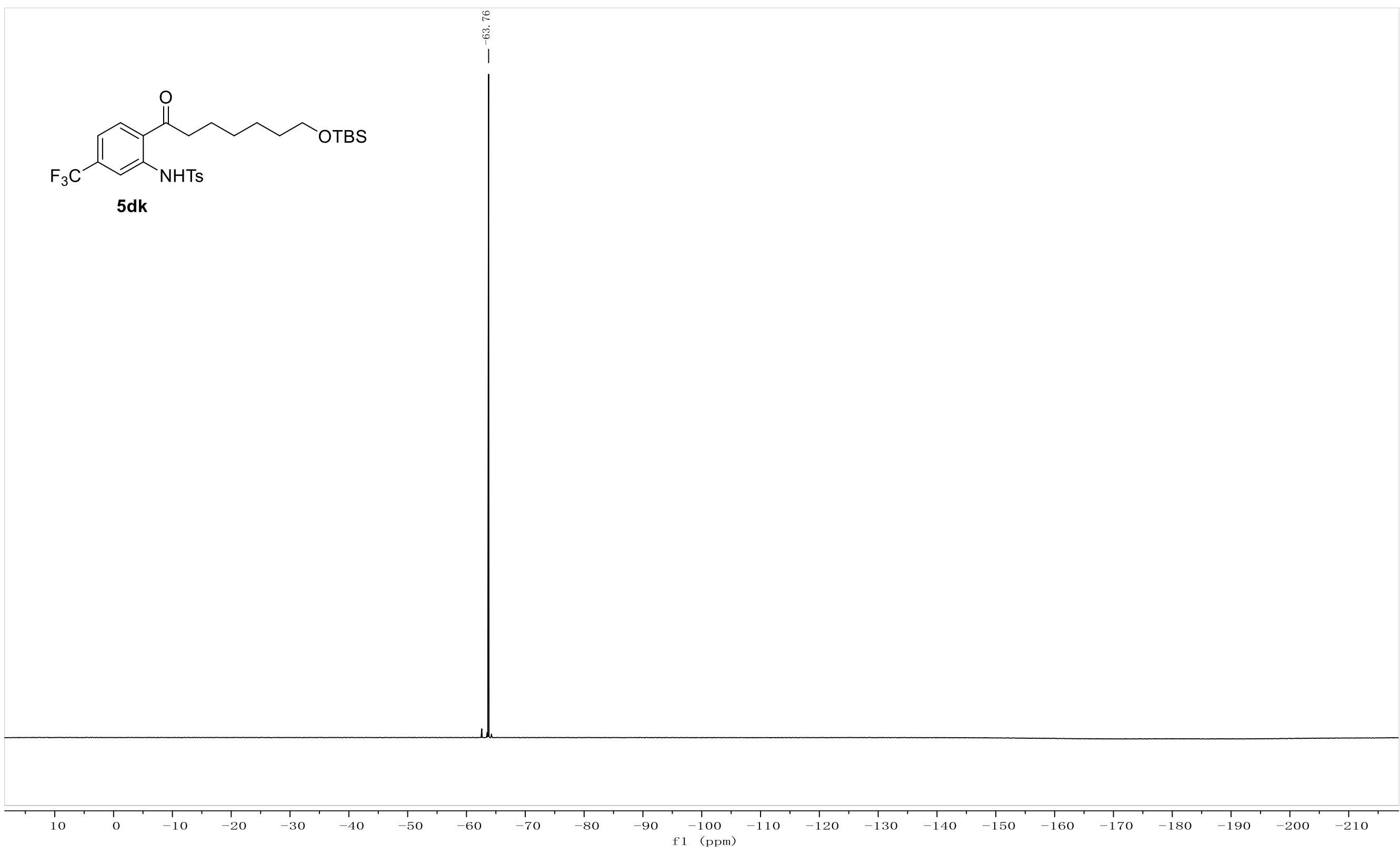


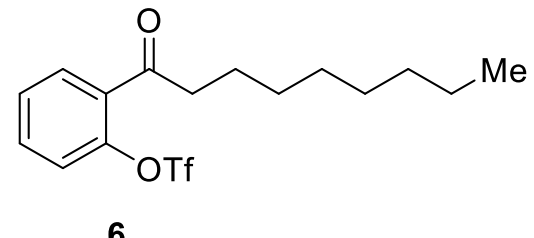

6

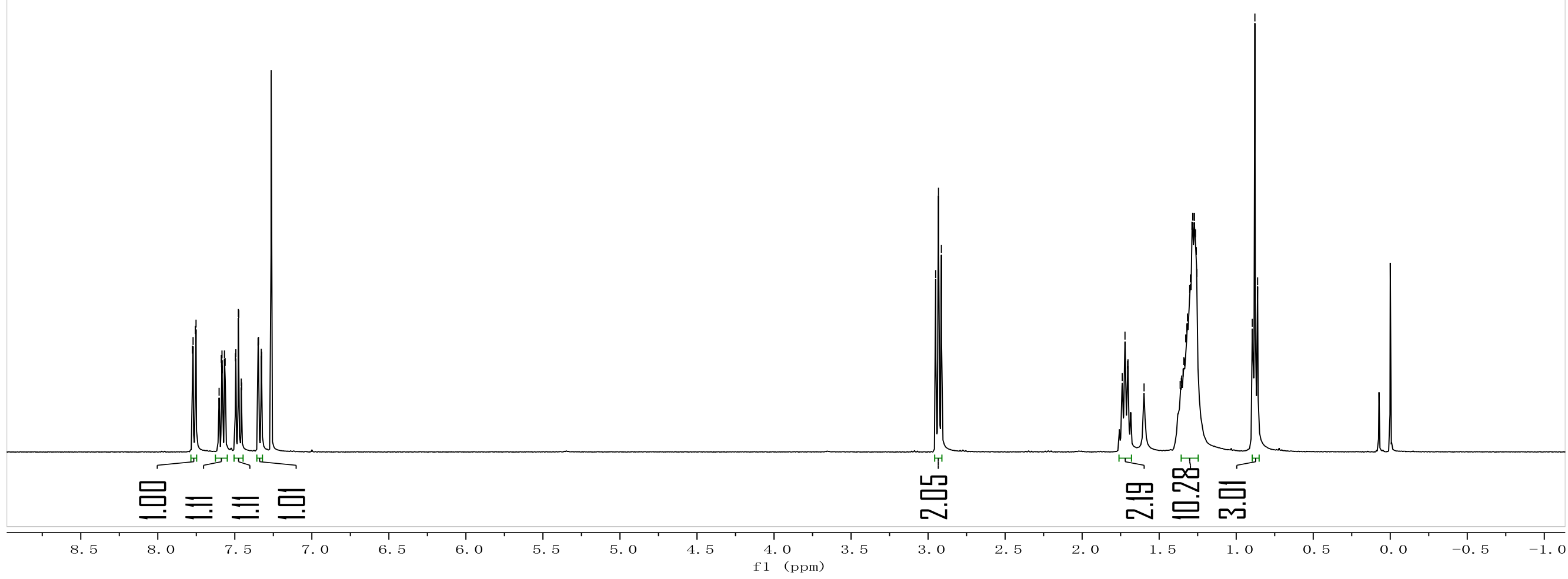


${ }^{13} \mathrm{C}$ NMR-spectrum $\left(101 \mathrm{MHz}, \mathrm{CDCl}_{3}\right)$ of $\mathbf{6}$
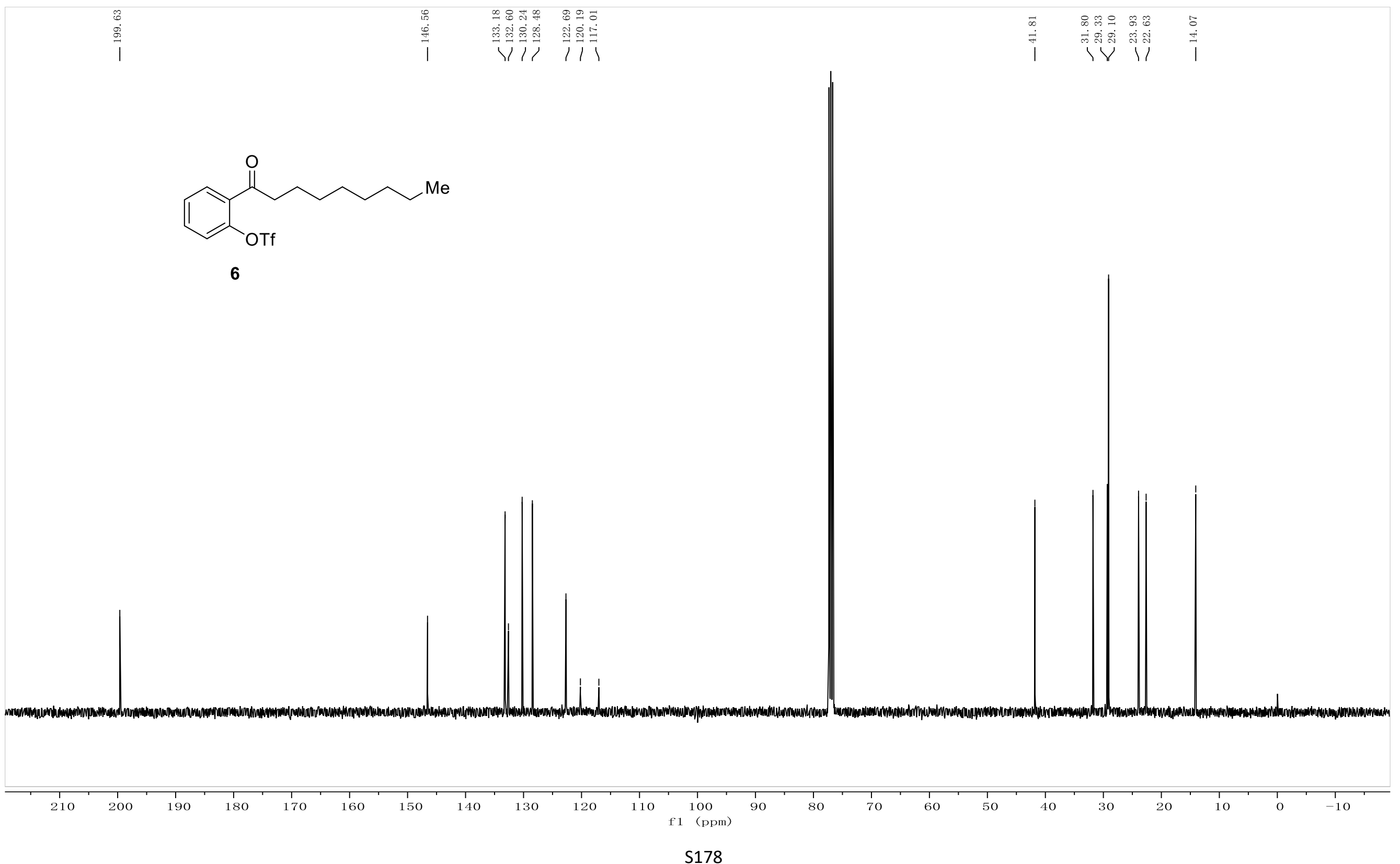
${ }^{19} \mathrm{~F}$ NMR-spectrum $\left(376 \mathrm{MHz}, \mathrm{CDCl}_{3}\right)$ of 6

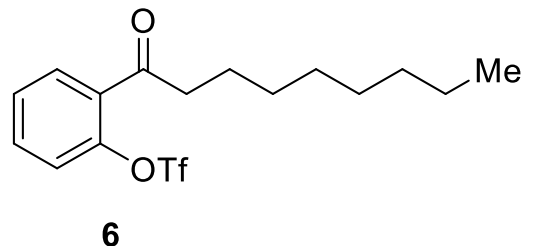

6

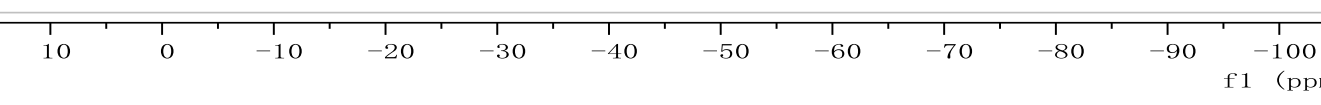


${ }^{1} \mathrm{H}$ NMR-spectrum (400 MHz, $\mathrm{CDCl}_{3}$ ) of 7

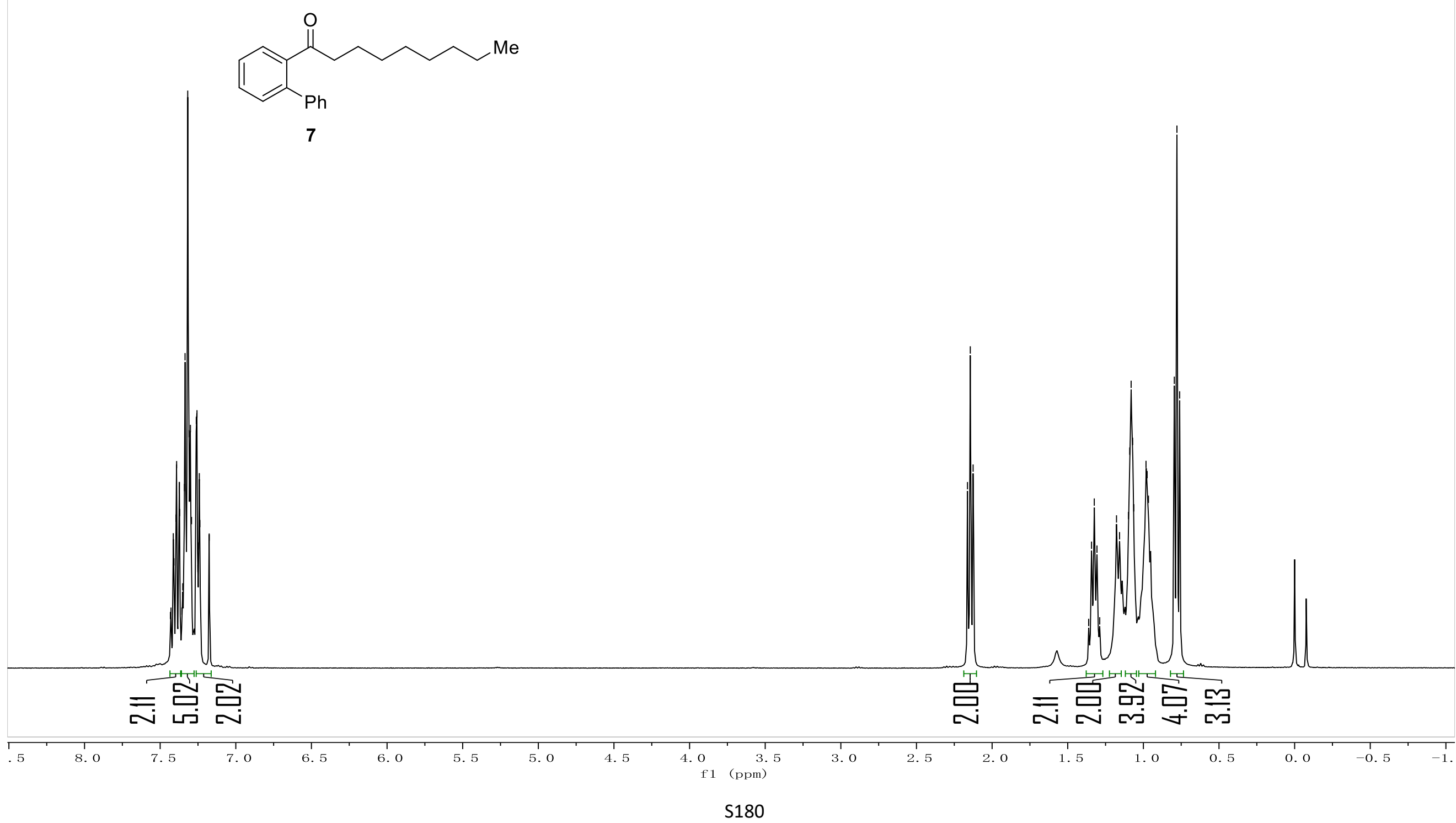


${ }^{13} \mathrm{C}$ NMR-spectrum $\left(126 \mathrm{MHz}, \mathrm{CDCl}_{3}\right)$ of 7
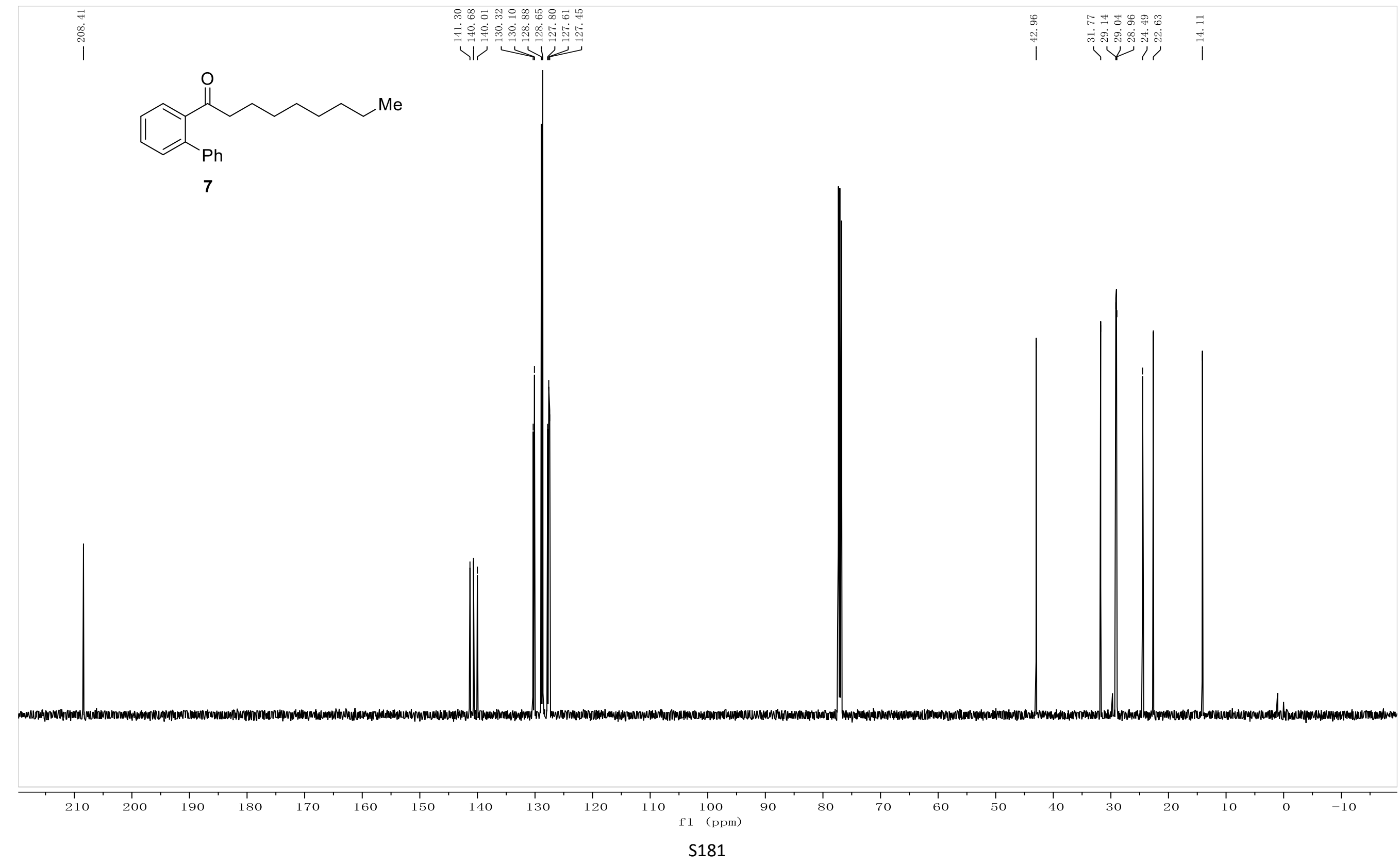
${ }^{1} \mathrm{H}$ NMR-spectrum $\left(500 \mathrm{MHz}, \mathrm{CDCl}_{3}\right)$ of 9
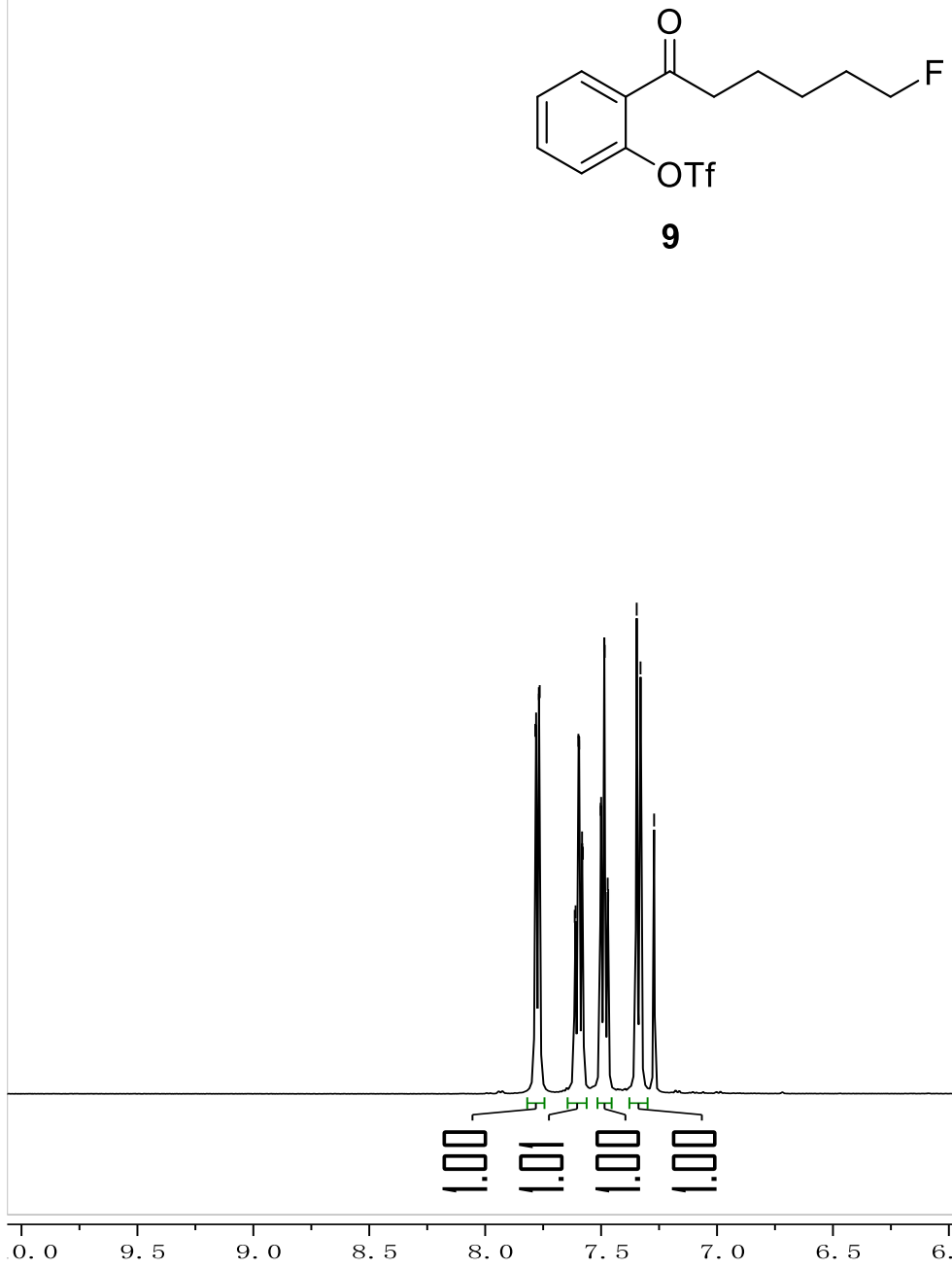

흐 管 总总总 
${ }^{13} \mathrm{C}$ NMR-spectrum $\left(126 \mathrm{MHz}, \mathrm{CDCl}_{3}\right)$ of 9

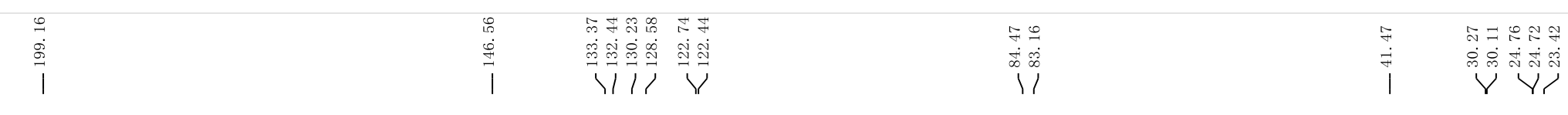

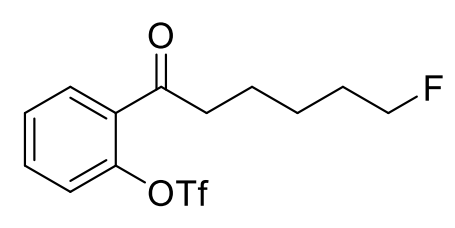

9

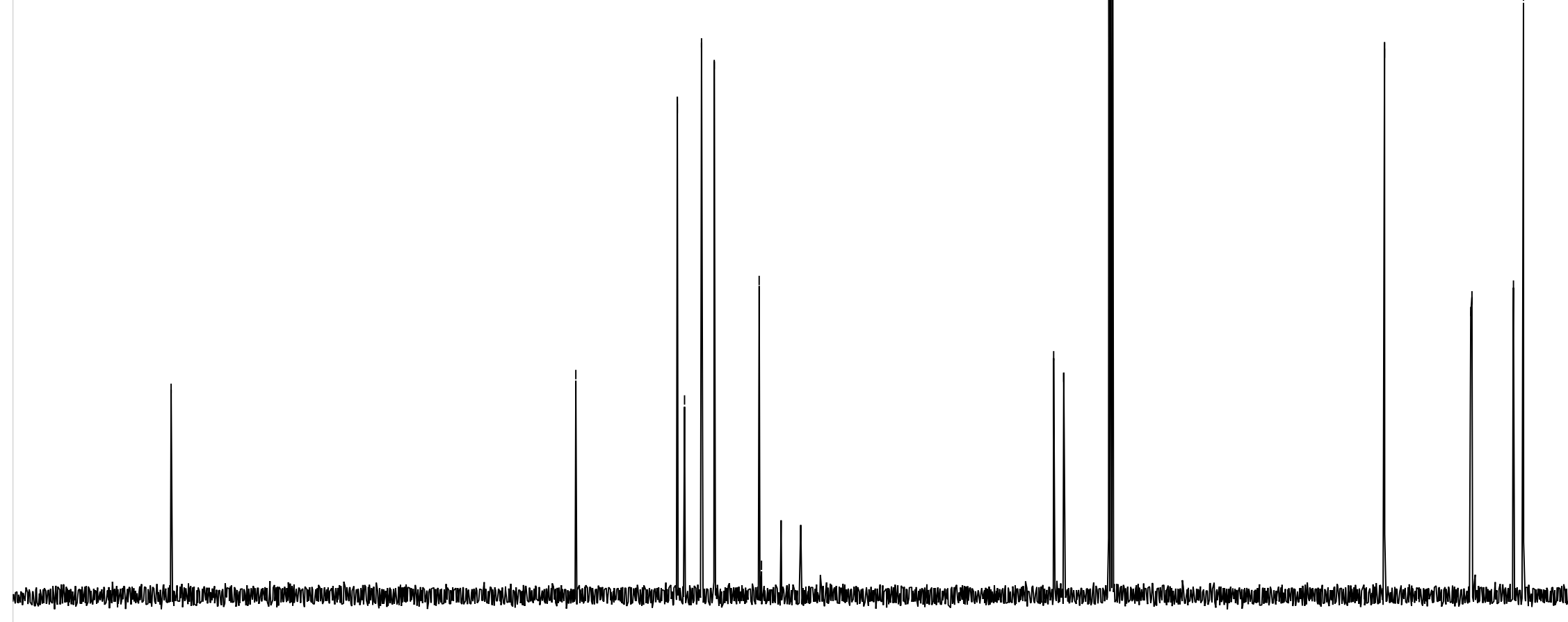

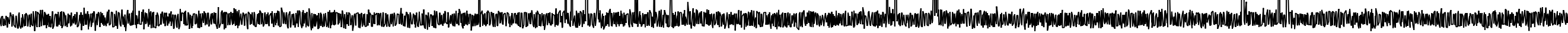

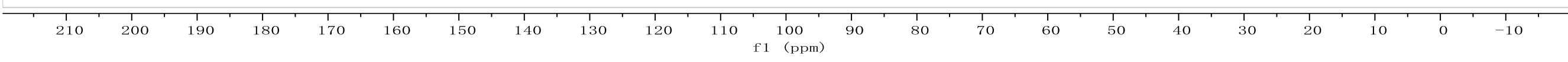


${ }^{19} \mathrm{~F}$ NMR-spectrum $\left(471 \mathrm{MHz}, \mathrm{CDCl}_{3}\right)$ of 9
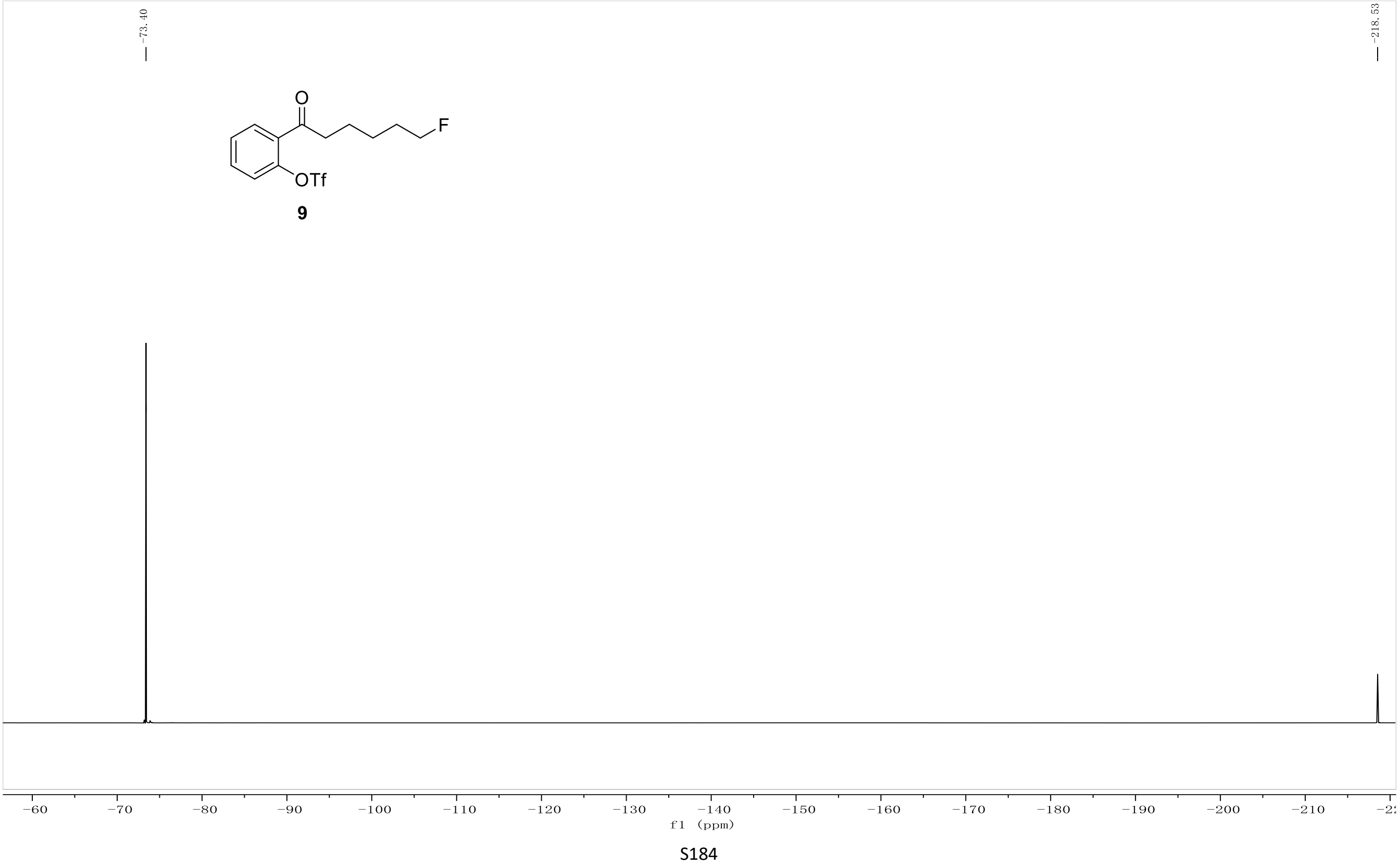
${ }^{1} \mathrm{H}$ NMR-spectrum $\left(400 \mathrm{MHz}, \mathrm{CDCl}_{3}\right)$ of $\mathbf{1 0}$

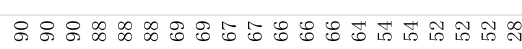

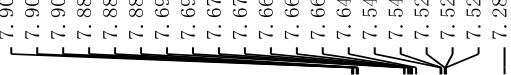
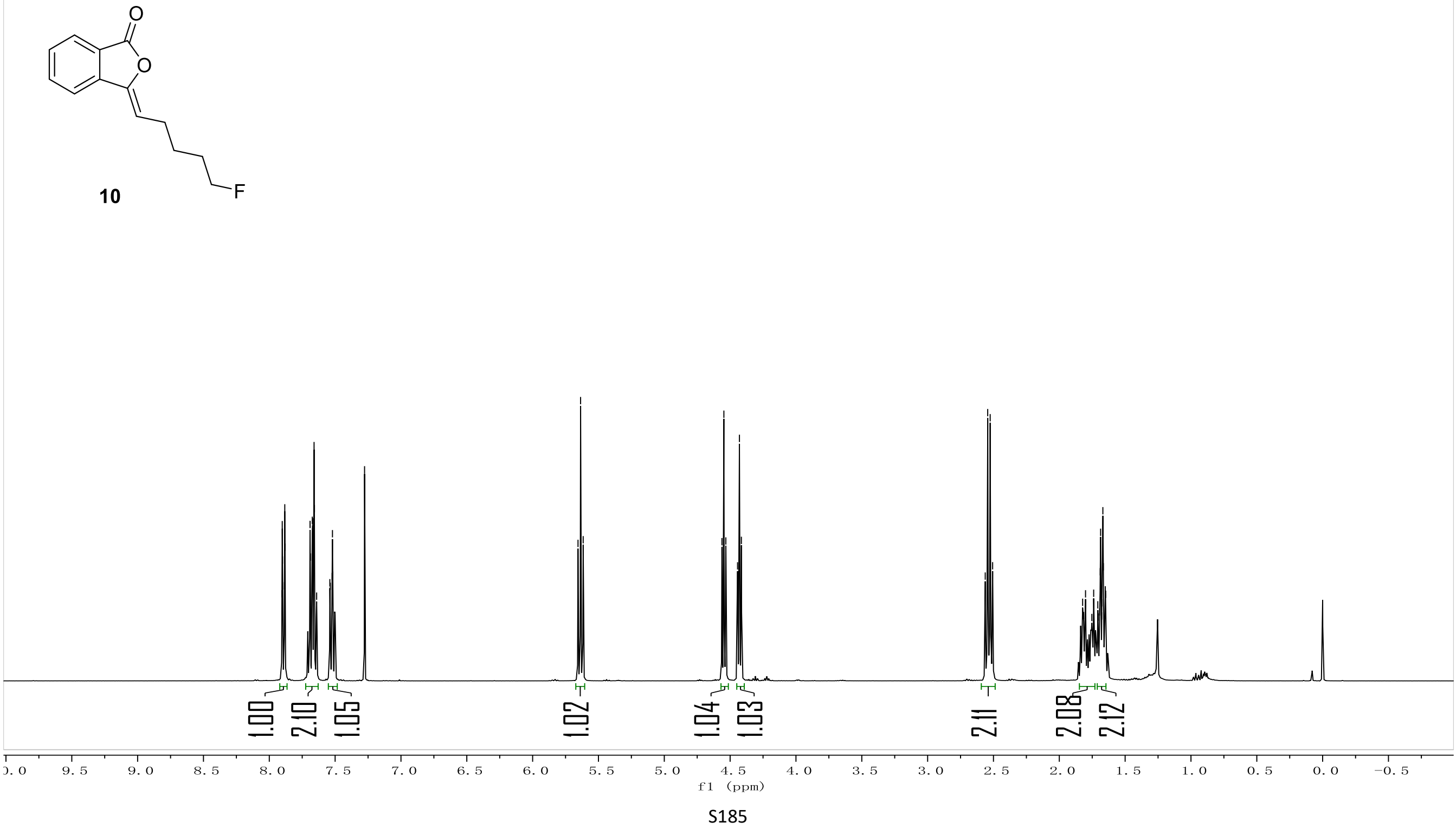
${ }^{13} \mathrm{C}$ NMR-spectrum $\left(101 \mathrm{MHz}, \mathrm{CDCl}_{3}\right)$ of $\mathbf{1 0}$

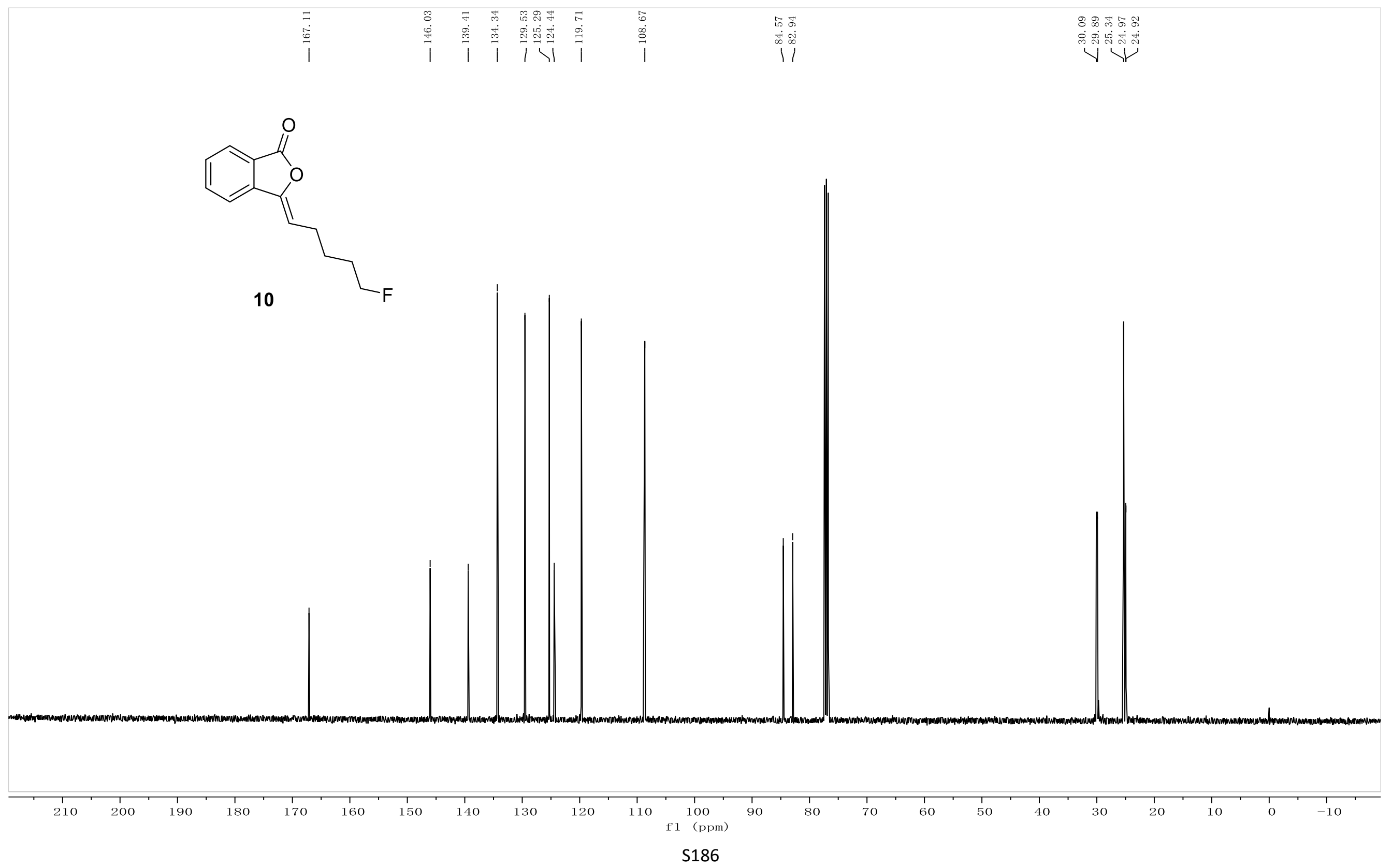


${ }^{19} \mathrm{~F}$ NMR-spectrum $\left(471 \mathrm{MHz}, \mathrm{CDCl}_{3}\right)$ of $\mathbf{1 0}$
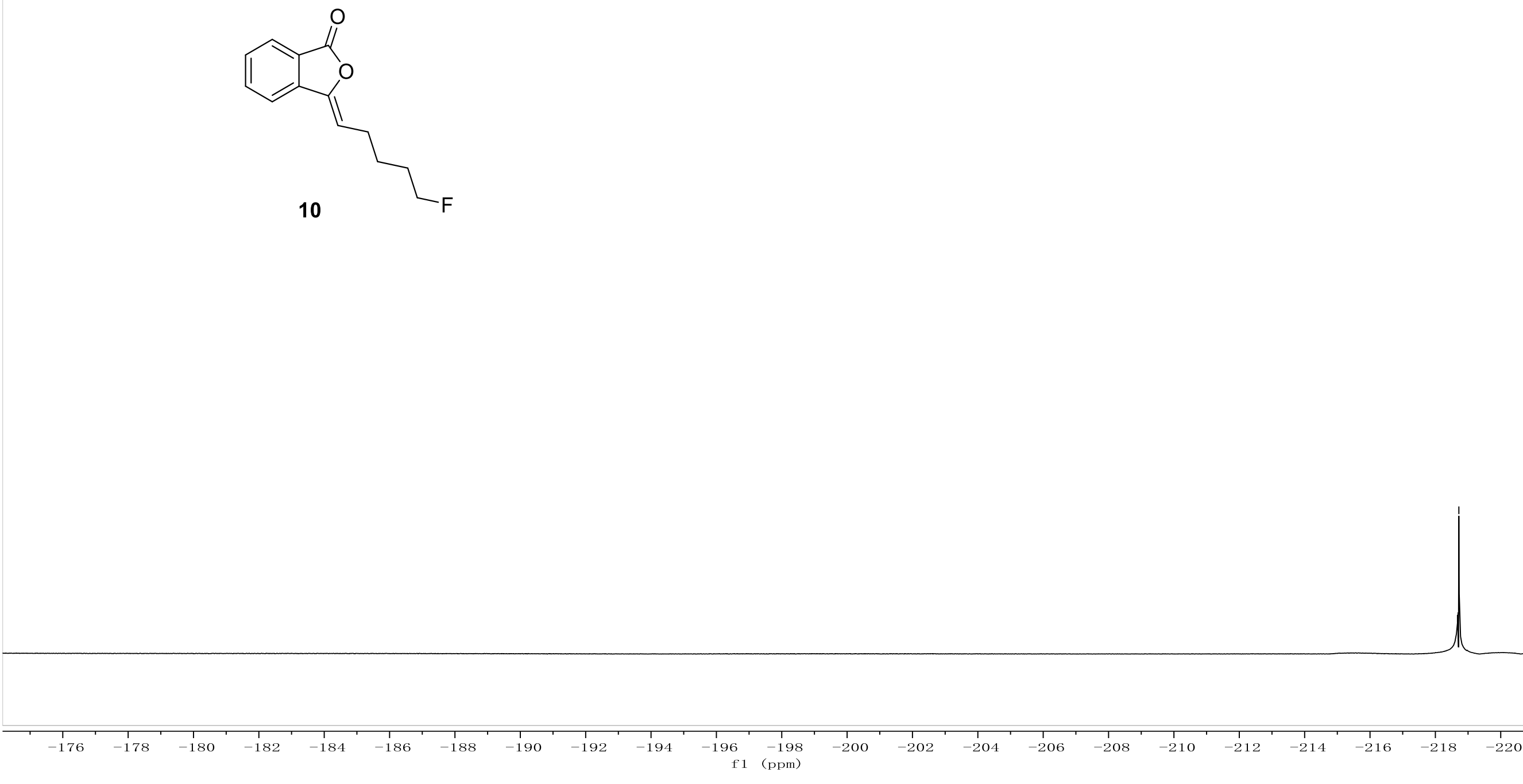
${ }^{1} \mathrm{H}$ NMR-spectrum $\left(500 \mathrm{MHz}, \mathrm{CDCl}_{3}\right)$ of 14

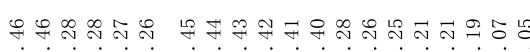

$\int_{\infty}^{\infty} \underbrace{\infty} \underbrace{\infty}$

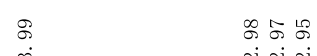

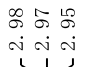

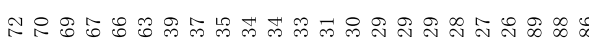

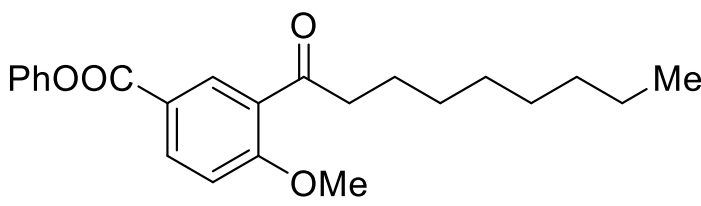

14

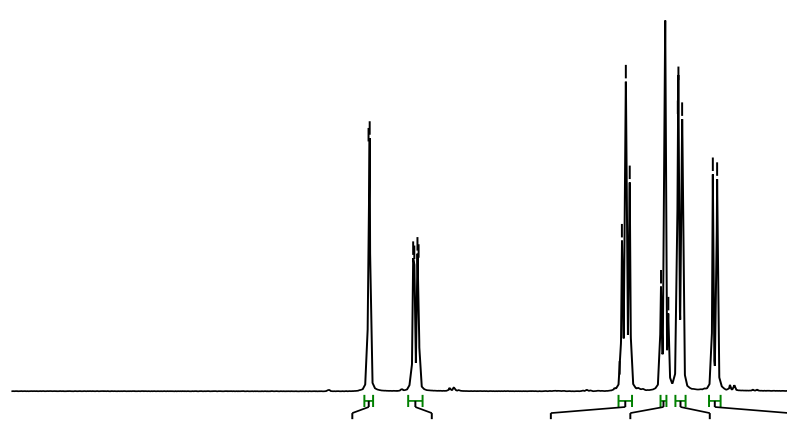

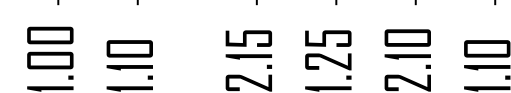

骂点吕

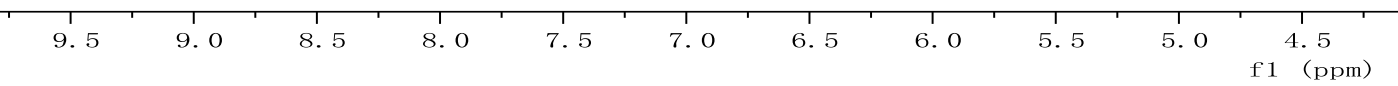


${ }^{13} \mathrm{C}$ NMR-spectrum $\left(126 \mathrm{MHz}, \mathrm{CDCl}_{3}\right)$ of $\mathbf{1 4}$

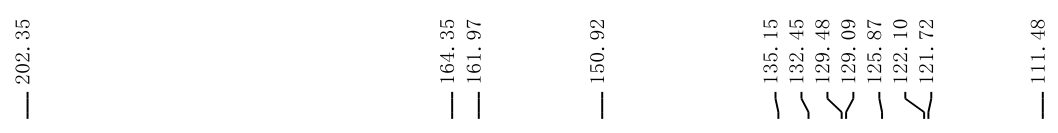

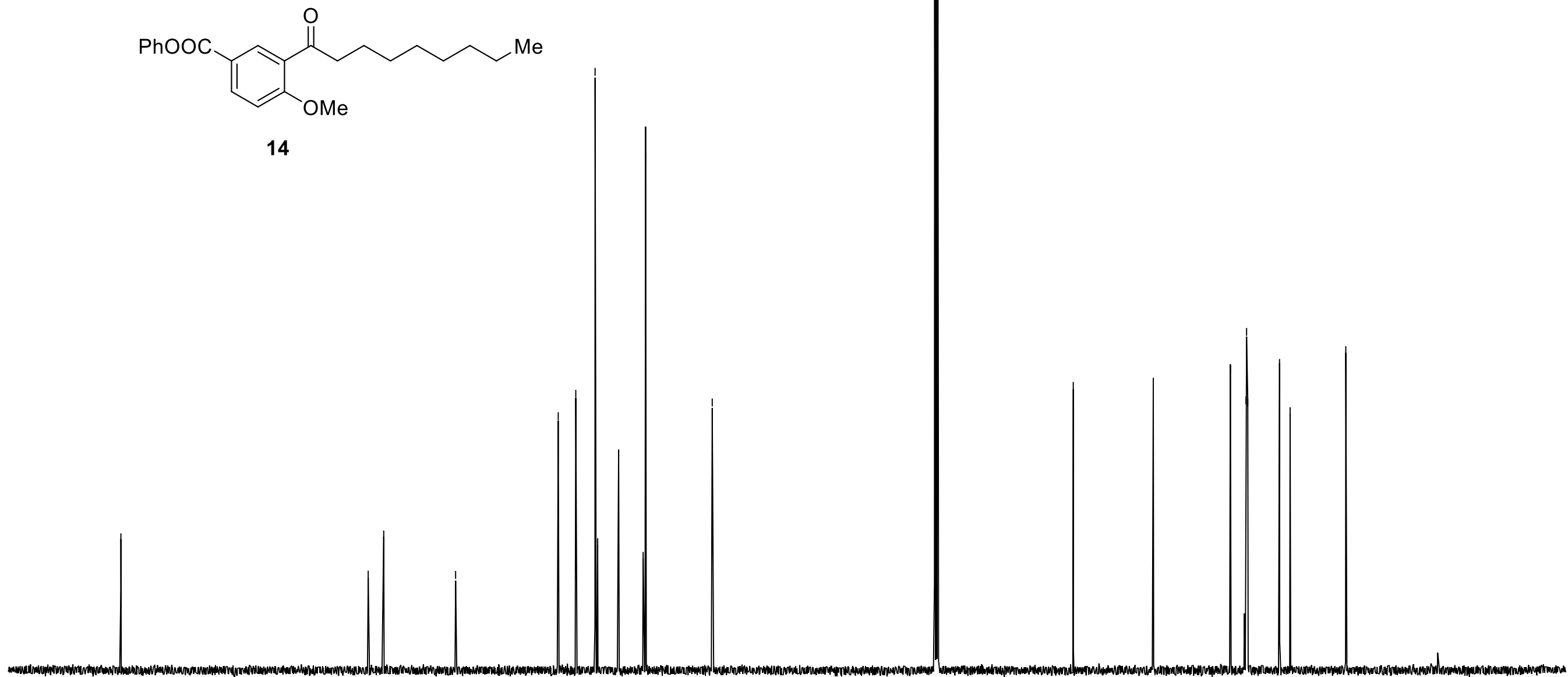

14

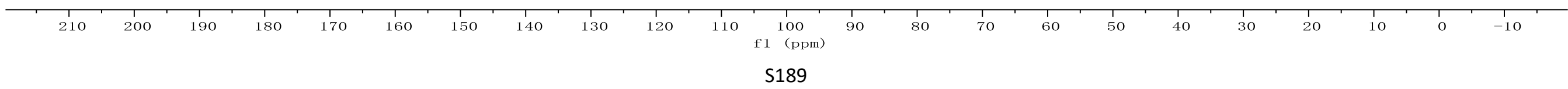


${ }^{1} \mathrm{H}$ NMR-spectrum (400 MHz, $\mathrm{CDCl}_{3}$ ) of $\mathbf{1 1}$

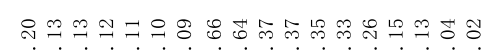

$\underbrace{\infty}$

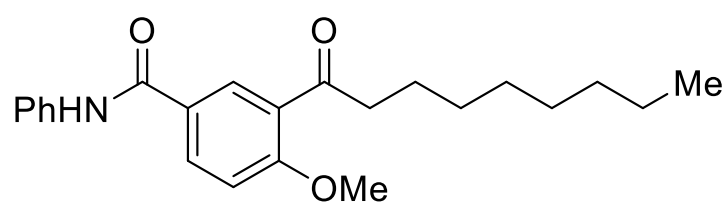

11

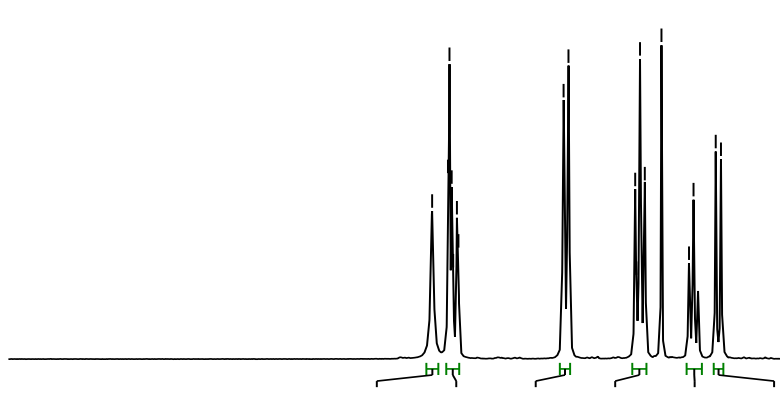

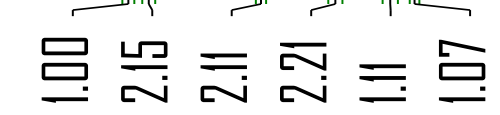

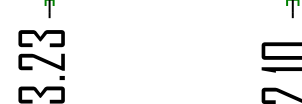

एक

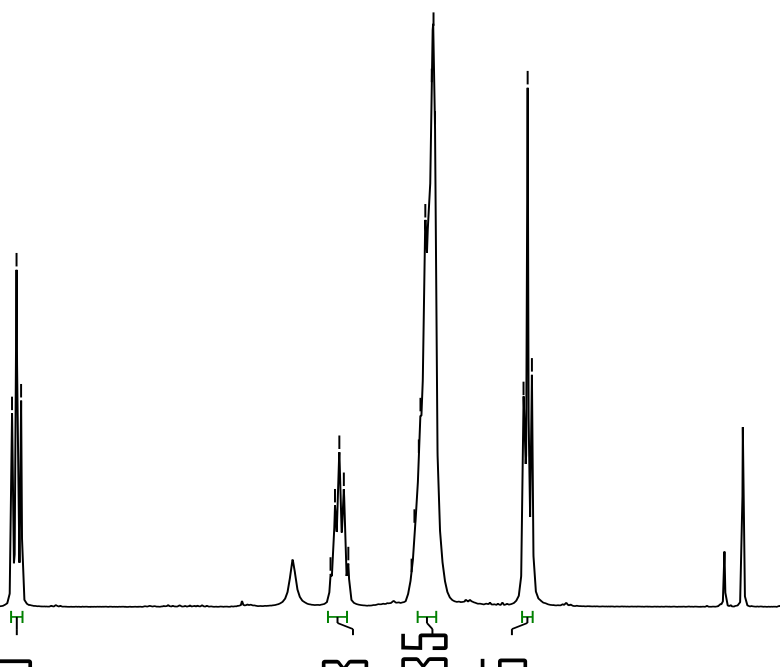

미 क्यं

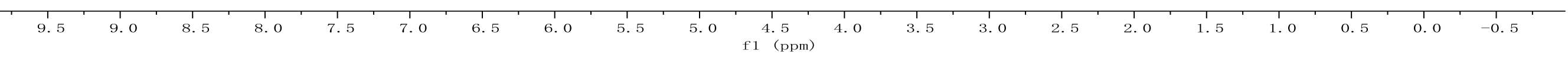


${ }^{13} \mathrm{C}$ NMR-spectrum (126 MHz, $\mathrm{CDCl}_{3}$ ) of $\mathbf{1 1}$

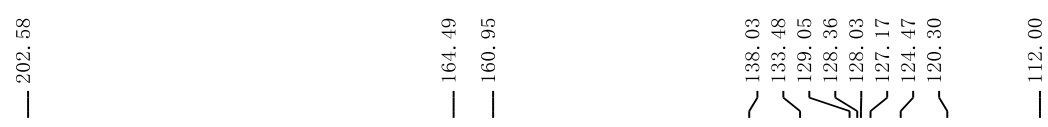

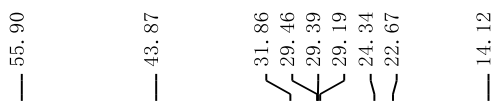<smiles>CCCCCCCCC(=O)c1cc(C(=O)Nc2ccccc2)ccc1OC</smiles>
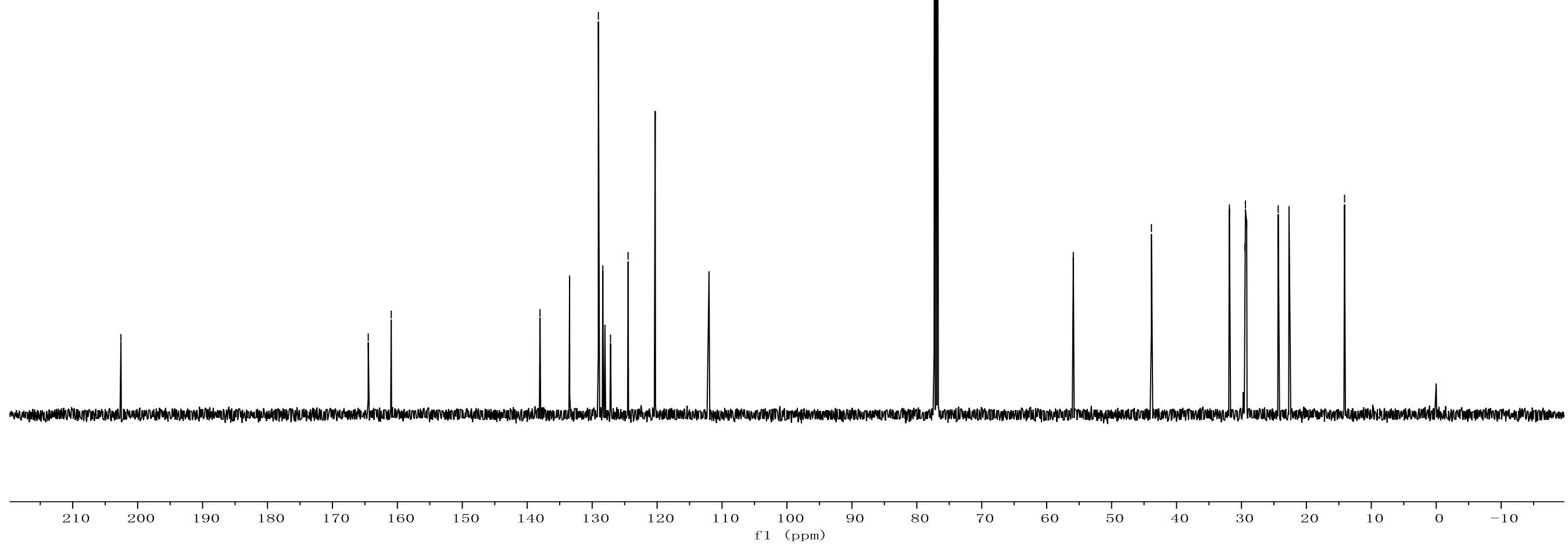
${ }^{1} \mathrm{H}$ NMR-spectrum (400 MHz, $\mathrm{CDCl}_{3}$ ) of $\mathbf{1 2}$

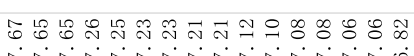

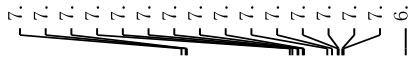

PhNHTs

12

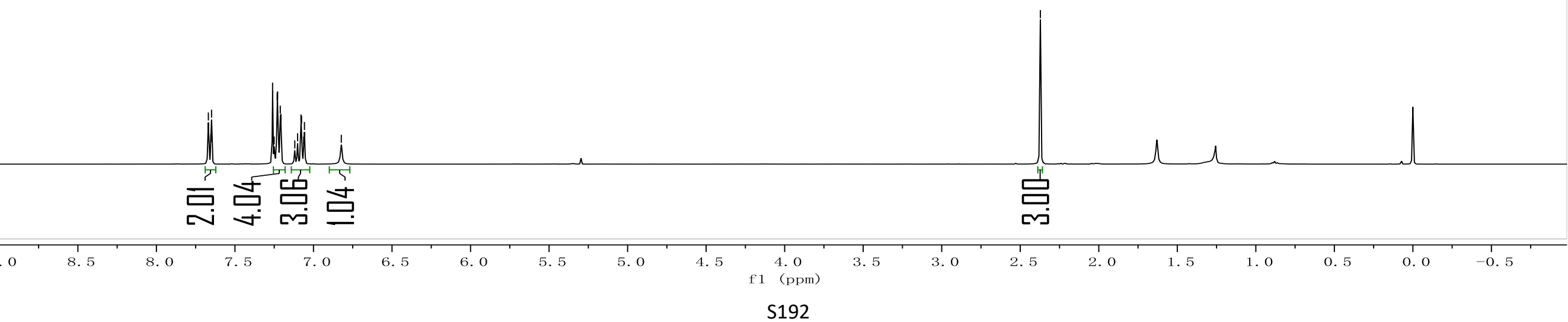


${ }^{13} \mathrm{C}$ NMR-spectrum $\left(101 \mathrm{MHz}, \mathrm{CDCl}_{3}\right)$ of $\mathbf{1 2}$

PhNHTs
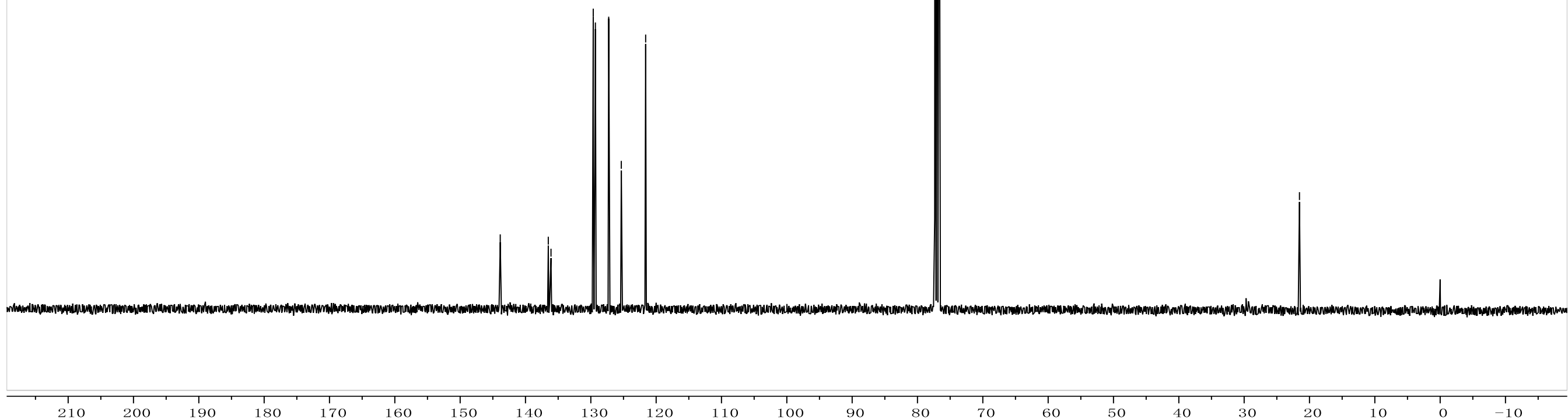